\title{
Impact of Tidal turbine support structures on realizable turbine farm power
}

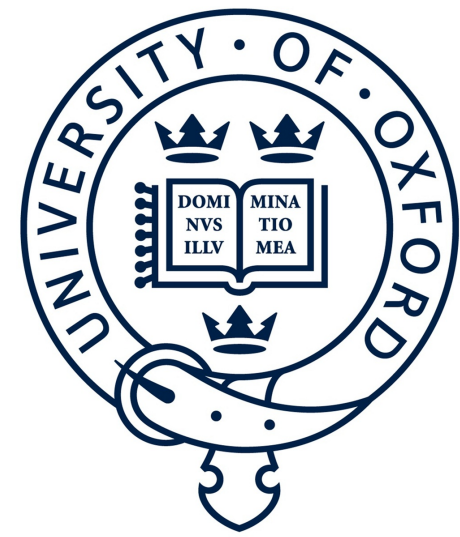

Subhash Muchala

St. Edmund Hall

University of Oxford

A thesis submitted for the degree of

Doctor of Philosophy

Trinity 2017 


\author{
Abstract \\ Impact of Tidal turbine support structures on realizable turbine farm power \\ A thesis submitted for the degree of Doctor of Philosophy \\ Subhash Muchala \\ St. Edmund Hall, Oxford \\ Trinity 2017
}

This thesis discusses the importance of tidal turbine support structures through analytical and computational modelling. A head-driven analytical channel model was first developed to determine the sensitivity of the flow to the presence and type of support structures. It showed that there was a significant potential reduction in farm power output even when only considering approximate force coefficients for rotor and support structure. To confirm these findings, computational simulations were performed on a full-scale turbine to obtain more accurate force coefficients considering full rotor-support structure interactions.

The flow interaction effects between the rotor and its support structure were studied using Computational Fluid Dynamics (CFD) for different support structure shapes for a range of tidal velocities including the power-capping zone. The integrated rotor force coefficients were higher in the presence of the cylindrical support structure than the elliptical support due to the higher opposing thrust from the cylinder in the channel redirecting the flow and increasing the flow velocity over the top half of the rotor. The presence of rotor caused a drop in the stream-wise forces on the support structure. The amplitude of the stream-wise sectional forces along the support structure height was lower in the case of an elliptical than a circular cylinder due to more streamlined shape of the ellipse.

At device scale, the computational model was used to study the turbine performance in the power-capping zone by pitching the blades to feather. The influence of pitch-tofeather power-capping strategy was examined by studying the forces and angle of attack on the turbine blades, and the wake at three different blade pitch angles. Increasing blade pitch angle resulted in a significant drop in the average load on the blade. Also since the tidal channel flow has a shear in its velocity profile, the influence of shear on turbine performance was studied by comparing it to the same turbine in a uniform flow.

The analytical channel flow model was used to investigate the characteristics of tidal stream energy extraction for large tidal farms deployed in tidal channels with specific focus on the limitations to realizable farm power due to turbine support structure drag and constraints on volume flow rate reduction. The force coefficients dataset from computational modelling was used to obtain a better estimate of the farm power output. Support structures were seen to contribute significantly to the overall resistive force in the channel and thus reduce the overall flow rates in the channel, leading to losses in realizable power. Over a wide range of channel characteristics, realistic levels of support structure drag lead to up to a $10 \%$ reduction in realizable power, and an associated reduction in the number of turbines that can be economically installed. 


\section{Acknowledgements}

I would like to thank my supervisor Prof. Richard Willden for his guidance and encouragement throughout my DPhil and also for his suggestions about the thesis.

Many thanks to my colleagues in the Civil Engineering group with whom I had many interesting discussions during my DPhil, and particularly Chris and Aidan for their valuable inputs to this thesis.

I would like to thank the Oxford Martin School for their support towards my DPhil and to St Edmund Hall for providing financial support to visit the OpenFOAM worshop in Portugal. I would like to thank the Advanced Research Computing group for letting me use the ARCUS super computer which helped in reducing the simulation time. I would also like to thank the IT department and the Engineering department for providing me various teaching opportunities in Oxford.

I am grateful to my parents and my sister for all the help and my friends, Varshita, for motivating me especially towards the final stages of my thesis, Meenakshi, Pranoy and Madhav who have been great friends and made my life at Oxford an enjoyable one.

It was a great experience to be part of the Civil Engineering group where I made many new friends, with whom I shared many meals at St. Johns in the last few years. 


\section{Contents}

LIST OF TABLES vi v vi

LIST OF FIGURES vii

CHAPTER 1: Introduction 1

1.1 Tides and tidal energy extraction . . . . . . . . . . . . . 3

1.2 Modelling of tidal farms . . . . . . . . . . . . . . . . . 7

1.3 Numerical modelling of rotors . . . . . . . . . . . . . . . . 11

1.3.1 Actuator Disc Model . . . . . . . . . . . . . . . . . . 11

1.3 .2 RANS-BEM . . . . . . . . . . . . . . 12

1.3.3 Actuator Line Model . . . . . . . . . . . . . . . . . . . . . . . . . 13

1.3.4 Blade resolved model . . . . . . . . . . . . . . . . 15

1.4 Free stream turbulence and shear flow . . . . . . . . . . . . . . 17

1.5 Support structures . . . . . . . . . . . . . . . . . . . . . . . . . . . . . . . . .

1.5.1 Support structure shapes . . . . . . . . . . . . 20

1.5.1.1 Circular cylinder . . . . . . . . . . . 20

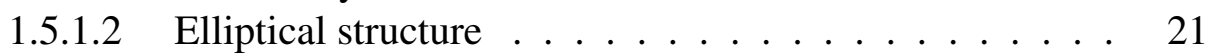

1.5.2 Effects of support structure on turbine system . . . . . . . 23

1.5.2.1 Thrust on the turbine system . . . . . . . . . 23

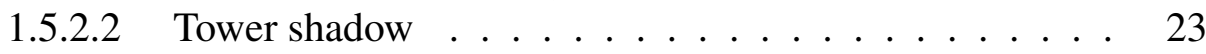

1.5.2.3 Flow velocity recovery ................ 24

1.6 Aim and Objectives . . . . . . . . . . . . . . . . . . 25

1.7 Thesis Outline . . . . . . . . . . . . . . . 26

CHAPTER 2: Farm power output analysis 29

2.1 Turbine Performance . . . . . . . . . . . . . . . . . . . . 29

2.2 Channel Dynamics . . . . . . . . . . . . . . . . . . 33

2.3 Power vs thrust . . . . . . . . . . . . . . . . . . 39

2.4 Available power in a volume flow rate reduction constrained flow . . . . . 44

2.5 Influence of support structure drag coefficient . . . . . . . . . . . . . 46

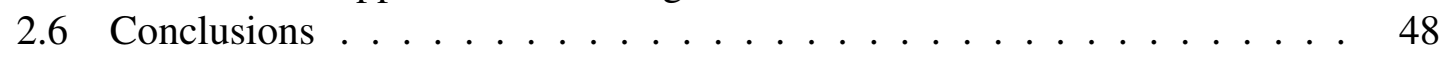


CHAPTER 3: Numerical Methods

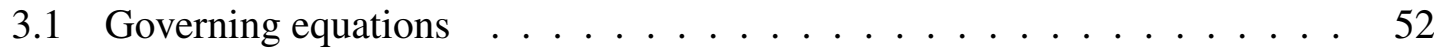

3.2 Discretization of the transport equation . . . . . . . . . . . 53

3.2.1 Spatial Discretization . . . . . . . . . . . . . . 54

3.2.1.1 Convection term .............. 54

3.2.1.2 First order schemes . . . . . . . . . . . . 54

3.2.1.3 Second order schemes ............ . . 55

3.2.1.4 Diffusion term . . . . . . . . . . . . 58

3.2.2 Temporal Discretization ................ 59

3.2.2.1 Euler Implicit scheme . . . . . . . . . . . . . . . . 60

3.2.2.2 Backward time difference scheme . . . . . . . . . 60

3.3 Turbulence Modelling . . . . . . . . . . . . . . . . . . 61

3.4 Wall Functions . . . . . . . . . . . . . . . . . . 67

3.5 Simulating Rotor motion . . . . . . . . . . . . . . 68

3.5.1 Mesh motion . . . . . . . . . . . . . . 68

3.5.2 Multiple Reference Frame . . . . . . . . . . . . . . . . . 69

3.6 Boundary Conditions . . . . . . . . . . . . . . . . . 70

CHAPTER 4: Model rotor and support structure validation 72

4.1 Cylindrical monopile . . . . . . . . . . . . . . . . . . 73

4.1.1 Cylinder 2D ....................... 74

4.1.2 Cylinder 3D . . . . . . . . . . . . . . . 78

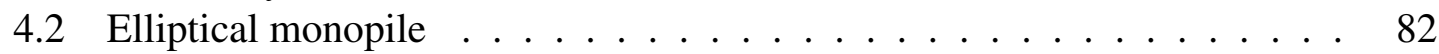

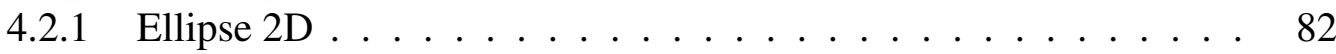

4.2.2 Comparison between circular cylinder and ellipse . . . . . . . . 84

4.2 .3 Ellipse 3D . . . . . . . . . . . . . . . . . . . . . 86

4.3 Model scale rotor . . . . . . . . . . . . . . . . . . 88

4.3 .1 Airfoil 2D . . . . . . . . . . . . . . . 89

4.3.2 Turbine Rotor - Multiple Reference Frame approach . . . . . . . 96

4.3 .3 Geometry .......................... 97

4.3.4 Computational domain and boundary conditions . . . . . . . . . . 99

4.3.5 Blocking strategy . . . . . . . . . . . . . . . . 100

4.3.6 Inlet parameters . . . . . . . . . . . . . . . 101

4.3.7 Mesh independence study . . . . . . . . . . . . . . . . 102

4.3.8 Inlet turbulence dependency . . . . . . . . . . . . . . . . . 104

4.3.9 Effect of tip speed ratio . . . . . . . . . . . . . . . . 106

4.3 .10 Comparison with experiment . . . . . . . . . . . . . . 109

4.3.11 Unsteady mesh rotation comparison . . . . . . . . . . . . . . 113

4.4 Summary ... . . . . . . . . . . . . . . . . . 114 
CHAPTER 5: Blade pitching in the power-capping zone 117

5.1 Introduction . . . . . . . . . . . . . . . . . . 117

5.2 Turbine rotor description . . . . . . . . . . . . . . . . 118

5.2.1 Geometry and domain . . . . . . . . . . . . . 118

5.2 .2 Mesh ....................... 119

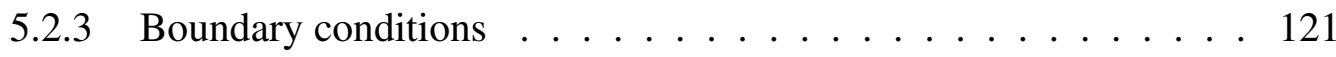

5.2.3.1 Uniform flow . . . . . . . . . . . . . . . 121

5.2.3.2 Shear flow ................. 121

5.3 Flow physics in uniform and shear flow ............ 123

5.3.1 Power and thrust coefficients of the rotor . . . . . . . . . . 123

5.3.2 Blade forces . . . . . . . . . . . . . . . 125

5.3 .3 Wake comparison ........................ 130

5.3.3.1 Velocity .................. 130

5.3.3.2 Turbulence intensity . . . . . . . . . . . 130

5.4 Blade pitching in the power-capping zone . . . . . . . . . . . . 135

5.4.1 Simulation strategy . . . . . . . . . . . . . 136

5.4.2 Power and thrust coefficients of the rotor . . . . . . . . . . . . 138

5.4 .3 Blade forces . . . . . . . . . . . . . . . . 139

5.4 Wake comparison ...................... 147

5.5 Conclusions . . . . . . . . . . . . . . . . . 152

CHAPTER 6: Influence of support structures 154

6.1 Introduction . . . . . . . . . . . . . . . . . . . . . . . . . . . . . 154

6.2 Turbine rotor description . . . . . . . . . . . . . . . 155

6.2.1 Computational domain . . . . . . . . . . . . 155

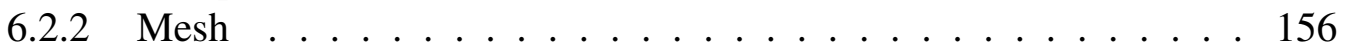

6.2 .3 Boundary conditions . . . . . . . . . . . . . 157

6.3 Flow physics in the presence of support structure . . . . . . . . . . 158

6.3.1 Power and thrust coefficients of the rotor . . . . . . . . . . . . 158

6.3 .2 Sectional blade forces . . . . . . . . . . . . . . . . . . . . 162

6.4 Forces on support structure . . . . . . . . . . . . . . . . . 167

6.4.1 Support structure sectional force variation . . . . . . . . . . . 168

6.4.2 Mean sectional force along length of the support structure . . . . 171

6.5 Wake comparison . . . . . . . . . . . . . . . . 172

6.6 Conclusions .......................... 175

CHAPTER 7: Reassessment of farm power output 177

7.1 Introduction . . . . . . . . . . . . . . . . . 177

7.2 Turbine performance . . . . . . . . . . . . . . 178

7.3 Channel power output . . . . . . . . . . . . . . . . . . 180

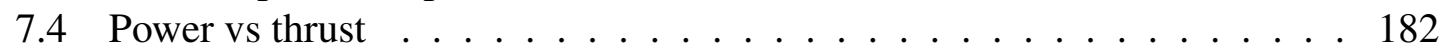

7.5 Available power in a volume flux reduction constrained flow . . . . . . 186 
7.6 Influence of support structure drag . . . . . . . . . . . . . . 190

7.7 Conclusion . . . . . . . . . . . . . . . . . . . . 191

CHAPTER 8: Conclusions 194

8.1 Conclusions ... . . . . . . . . . . . . . 195

8.1.1 Influence of shear flow and blade pitching on turbine performance 195

8.1.2 Rotor-support structure interaction . . . . . . . . . . . 196

8.1.3 Tidal farm power output . . . . . . . . . . . . . . . . 198

8.2 Future Work . . . . . . . . . . . . . . . . . . . . . . . . 199 


\section{List of Tables}

2.1 Farm power output dependency on $F r_{w} \ldots \ldots \ldots \ldots \ldots \ldots$

2.2 Farm power output dependency on $\mathrm{L} / \mathrm{h} \ldots \ldots \ldots \ldots \ldots$

4.1 Spanwise mesh dependency . . . . . . . . . . . . . . . . . . . 79

4.2 Comparison with experimental and CFD data $\ldots \ldots \ldots \ldots$. . . . 81

4.3 Influence of Aspect Ratio on Ellipse . . . . . . . . . . . . . . 83

4.4 Comparison with experiment $\ldots \ldots \ldots \ldots$. . . . . . . . . 87

4.5 Turbine blade spanwise distribution $\ldots \ldots \ldots \ldots \ldots$

4.6 Mesh Independence study at TSR $=6 \ldots \ldots \ldots$

4.7 Free stream turbulence . . . . . . . . . . . . . . . . . . . . . . 104

5.1 Rotor force coefficients for three different blade pitch angles and flow speeds 138

6.1 Mesh cell count . . . . . . . . . . . . . . . . . . . . . . . . . 157

6.2 Time-averaged rotor force coefficients in the presence of different support structures at $10^{\circ}$ blade pitch angle . . . . . . . . . . . . . 159

6.3 Values of the mean stream-wise force coefficients of different support structures behind the rotor at $10^{\circ}$ blade pitch angle . . . . . . . . 167

7.1 Force coefficients obtained from CFD for three different tip speed ratios in the presence of different support structures _ . . . . . . . . . 178

7.2 Farm power output dependency on $F r_{w} \ldots \ldots \ldots \ldots \ldots$

7.3 Farm power output dependency on $\mathrm{L} / \mathrm{h} \ldots \ldots \ldots$

7.4 Influence of support structure drag on $C_{P_{c}}$ with a $10 \%$ volume flow rate reduction constraint for different $F r_{w} \ldots \ldots \ldots \ldots$

7.5 Influence of support structure drag on $C_{P c}$ with a $10 \%$ volume flow rate reduction constraint for different $\mathrm{L} / \mathrm{h} \ldots \ldots \ldots \ldots 1$ 


\section{List of Figures}

1.1 Tides generated due to gravitational forces involving sun, moon and earth

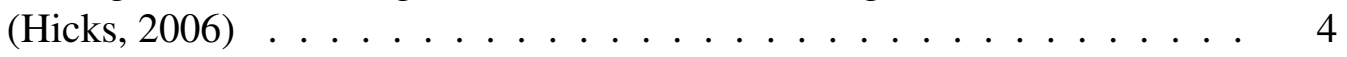

1.2 Types of tidal current turbines . . . . . . . . . . . . . 6

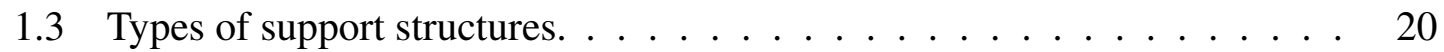

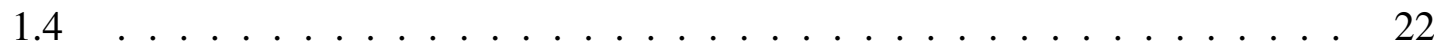

2.1 Power, $\mathrm{P}$, curve and Thrust, $\mathrm{T}$, curves for a turbine with different support structure drag coefficients, $0 \leq C_{D} \leq 1.8$, as a function of channel velocity, $U_{A}$; area ratio $\chi=0.2 . \ldots \ldots \ldots \ldots$

2.2 Hydrodynamic efficiency, $\eta$, curves for a turbine with different support structure drag coefficients, $0 \leq C_{D} \leq 1.8$, and area ratio $\chi=0.2$. The theoretical curve from LMADT is also plotted for comparison. Dashed lines indicate channel velocities. . . . . . . . . . . . . .

2.3 Volume flow rate, $\mathrm{Q}$, power, $\mathrm{P}$, and thrust, $\mathrm{T}$, curves plotted over one tidal cycle $\left(\chi=0.2, n=34, C_{D}=1.2, F r_{w}=0.478, L / h=125, C_{f}=\right.$

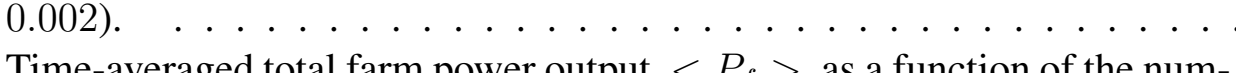

2.4 Time-averaged total farm power output, $\left\langle P_{f}\right\rangle$, as a function of the number of turbines installed and support structure drag coefficient. The dashed lines indicate the maximum channel velocity $\left(U_{A \max }\right)$ attained through the tidal cycle $\left(\chi=0.2, F r_{w}=0.478, L / h=125, C_{f}=0.002\right) \ldots \ldots$.

2.5 Farm performance, power-to-thrust, for various tidal amplitudes, and an unconstrained case for which turbine cut-in and rated speeds are neglected such that $P \propto U_{A}^{3}\left(L / h=125, \chi=0.2, C_{D}=1.2, C_{f}=0.002\right) \ldots \ldots$.

2.6 Channel power coefficient, $C_{P c}$, as a function of channel thrust coefficient, $C_{T c}$, for (a) $F r_{w}=0.673(a=0.125 m)$, (b) $F r_{w}=0.478(a=0.25 m)$, (c) $F r_{w}=0.338(a=0.5 m)$. The dashed lines indicate constraints on change in the maximum volume flow rate in the channel. $(L / h=$ $\left.125, C_{f}=0.002, \chi=0.2\right) \ldots \ldots \ldots \ldots \ldots \ldots$

2.7 Channel power coefficient, $C_{P c}$, as a function of channel thrust coefficient, $C_{T c}$, for (a) $L / h=75$, (b) $L / h=125$, (c) $L / h=175$. The dashed lines indicate constraints on change in the maximum volume flow rate in the channel. $\left(F r_{w}=0.478, C_{f}=0.002, \chi=0.2\right) \ldots \ldots \ldots \ldots \ldots$ 
2.8 The influence of maximum undisturbed channel velocity (due to different $F r_{w}$ ) on the time-averaged power output of the tidal farm, $\left\langle P_{f}\right\rangle$, for different support structure drag coefficients. $\left(L / h=125, C_{f}=0.002, \chi=\right.$

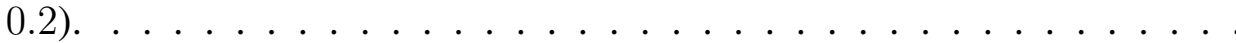

2.9 The influence of maximum undisturbed channel velocity (due to different $L / h$ ) on the time averaged power output of the tidal farm, $\left\langle P_{f}\right\rangle$, for different support structure drag coefficients. $\left(F r_{w}=0.478, C_{f}=0.002, \chi=\right.$

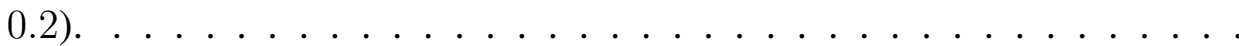

2.10 The influence of $F r_{w}$ on channel power coefficient, $C_{P c}$, as a function of the drag coefficient of the support structure with a $10 \%$ volume flow rate reduction constraint imposed $\left(\chi=0.2, L / h=125, C_{f}=0.002\right) . \quad$. . . .

2.11 The influence of $L / h$ on channel power coefficient, $C_{P c}$, as a function of the drag coefficient of the support structure with a $10 \%$ volume flow rate reduction constraint imposed $\left(\chi=0.2, F r_{w}=0.478, C_{f}=0.002\right) . \quad$. .

3.1 A simple representation of neighbouring cells, $\mathrm{P}$ and $\mathrm{N}$, in 2D. S is an area normal of cell P. $r_{1}$ and $r_{2}$ are the distances from cell centres to face (f) centre. . . . . . . . . . . . . . . . .

3.2 Variation of flux limiter $\Psi(r)$ with $r$ for various second order TVD schemes (Versteeg and Malalasekera, 2007). . . . . . . . . . . . . . . 57

4.1 Computational domain for simulating flow past a cylinder . . . . . . . . . 74

4.2 Mesh around the 2 D cylinder. . . . . . . . . . . . . . . . . 76

4.3 Profile of mean pressure coefficient on the cylinder surface using different meshes. . . . . . . . . . . . . . . . . 77

4.4 Visualization of pressure (a) and vorticity (b) distributions in unsteady, uni-directional flow past a cylinder at $R e=2 \times 10^{6}$. . . . . . . . . . . .

4.5 Comparison of pressure coefficient; CFD (Present work) at $\operatorname{Re}=2 \times 10^{6}$, CFD (URANS) at $\operatorname{Re}=1 \times 10^{6}$ by Catalano et al. (2003), Experimental at $\operatorname{Re}=3.6 \times 10^{6}$ by Achenbach (1968), Experimental at $\operatorname{Re}=1.2 \times 10^{6}$ by Warschauer and Leene (1971). . . . . . . . . . . . . . . . .

4.6 Comparison of skin friction coefficient; CFD (Present work) at $\operatorname{Re}=2 \times$ $10^{6}, \mathrm{CFD}(\mathrm{LES})$ at $\mathrm{Re}=1 \times 10^{6}$ by Catalano et al. (2003), Experimental at $\mathrm{Re}=3.6 \times 10^{6}$ by Achenbach (1968). . . . . . . . . . . . . . . 81

4.7 Mean pressure coefficient on the surface of ellipses of different aspect ratios. 83

4.8 Mean pressure coefficients at various angle of attack for elliptical cylinders of different elliptic aspect ratios. . . . . . . . . . . . . . . . . . . 85

4.9 Influence of angle of attack (AOA) on the mean drag and lift coefficients of various support structure shapes. . . . . . . . . . . . . . . 86

4.10 Comparison of Pressure coefficient (a) and Skin friction coefficient (b) between a cylinder and an ellipse. . . . . . . . . . . . . . . . 87

4.11 Thickened trailing edge (TE) of a NACA 63-821 airfoil . . . . . . . . . . 90 
4.12 Mesh around the airfoil . . . . . . . . . . . . . . . . . . . 91

4.13 Pressure coefficient comparison on different meshes at $8^{\circ}$ angle of attack. 92

4.14 Comparison between pressure coefficients $\left(C_{p}\right)$ predicted by CFD and XFOIL at different angles of attack (AOA). . . . . . . . . . . . . . . 94

4.15 Lift and drag coefficients of the airfoil for different angles of attack (AOA). 95

4.16 Computation domain with inner domain highlighted. . . . . . . . . . 99

4.17 Blocking for a $120^{\circ}$ wedge of the Inner Domain. . . . . . . . . . . . . . 100

$4.18 \mathrm{C}$ grid around the blade section at $r / R=0.8 \ldots \ldots . . \ldots 101$

4.19 Pressure coefficients at various sections for different mesh count. . . . . . 103

4.20 Pressure coefficients at different sections for various TI. . . . . . . . . . . 105

4.21 Skin friction coefficients at different sections for various turbulence intensities (TI). . . . . . . . . . . . . . . . . . . 106

4.22 Normalized turbulent kinetic energy TKE, $k / U_{\infty}^{2}$, at $r / R=0.5$ for various turbulence intensities (TI) . . . . . . . . . . . . . . . . 107

4.23 Pressure coefficients at different sections along the span for various tip speed ratios (TSR). . . . . . . . . . . . . . . . . 108

4.24 Skin friction coefficients at different sections along the span for various tip speed ratios (TSR). . . . . . . . . . . . . . . . . . . 109

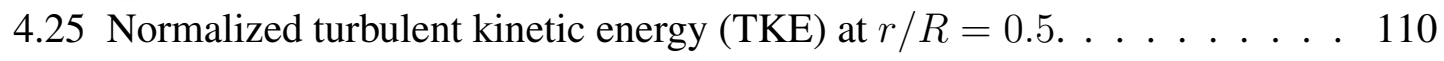

4.26 Blockage corrected thrust (a) and power (b) coefficients for various tip speed ratios. . . . . . . . . . . . . . . . . . . . . 112

4.27 Pressure coefficients at different sections of the blade obtained using different methods of modelling turbine rotation.

5.1 Dimensions of the computational domain. The flow direction is from left to right. . . . . . . . . . . . . . . . . . . . 118

5.2 Mesh distribution . . . . . . . . . . . . . . . . . . . 120

5.3 Velocity profiles in uniform and sheared flow. Both have a depth-averaged velocity of $2 \mathrm{~ms}^{-1}$. Rotor is located between $0.25 h$ and $0.75 h$ where $h$ is the depth of the channel. . . . . . . . . . . . . .

5.4 Power and thrust coefficients in uniform and shear flow at different tip speed ratios. The depth averaged velocity of $2 \mathrm{~ms}^{-1}$ is used for calculating the coefficients in both uniform and shear flow cases. . . . . . . . . . .

5.5 Blade force coefficients normalised by rotor force coefficients for uniform and shear flow over one turbine rotation; $0^{\circ}$ represents the top most point and $180^{\circ}$ represents the bottom most point. . . . . . . . . . .

5.6 Spanwise variation of approach angle, $\phi^{\circ}$, along the blade during a rotation cycle in uniform and shear flow. The depth averaged flow velocity is 2 $\mathrm{ms}^{-1}$ in both cases. Note the vertical asymmetry in the shear flow case. . . 126

5.7 Spanwise variation of angle of attack, $\alpha^{\circ}$, along the blade during a rotation cycle in uniform and shear flow. The depth averaged flow velocity is 2 $\mathrm{ms}^{-1}$ in both cases. . . . . . . . . . . . . . . . . . . . 127 
5.8 Angle of attack variation along the span of a turbine blade for uniform and shear flow at the top (solid) and bottom (dashed) position in a rotation cycle. 127

5.9 Spanwise axial force $\left(\mathrm{N} \mathrm{m}^{-1}\right)$ variation along the blade during a rotation cycle in uniform and shear flow. The depth averaged flow velocity is 2 $\mathrm{ms}^{-1}$ in both cases. . . . . . . . . . . . . . . . . . . . 128

5.10 Sectional force variation along the span of a turbine blade for uniform and shear flow, at the top and bottom position in a rotation cycle. . . . . . .

5.11 Normalised velocity deficit at different downstream locations in the near wake. $0.125 \mathrm{D}$ is the location of a potential support structure which will be discussed in the next chapter. In the shear flow case, the velocity is normalised based on the inlet shear profile. . . . . . . . . . . . .

5.12 Comparison of normalised axial velocity at lateral mid-section between the uniform flow case (a) and the shear flow case (b). In both the cases, the velocity is normalised with the depth averaged velocity of $2 \mathrm{~ms}^{-1}$. . .

5.13 Time-averaged cross-stream variation of axial velocity in the near wake for uniform (left) and shear (right) flow cases at three different locations downstream of the rotor plane. . . . . . . . . . . . . . .

5.14 Time-averaged cross-stream variation of turbulence intensity (TI) in the near wake for uniform (left) and shear (right) flow cases at three different locations downstream of the rotor plane. . . . . . . . . . .

$5.15 \mathrm{C}_{P}$ curves for four different blade pitch angles (blue lines), $\beta ; 0^{\circ}, 5^{\circ}, 10^{\circ}$ and $20^{\circ}$. The intersection points between the $\mathrm{C}_{P}$ curves and power control line (red) are the operating points. . . . . . . . . . . . .

5.16 Variation in power and thrust on the turbine rotor with channel flow speed. Each channel flow velocity corresponds to a different blade pitch angle of $0^{\circ}\left(2.37 \mathrm{~ms}^{-1}\right), 10^{\circ}\left(2.96 \mathrm{~ms}^{-1}\right)$ and $20^{\circ}\left(4.5 \mathrm{~ms}^{-1}\right)$. Also plotted is the axial root bending moment (BM) on a single blade for the three different blade pitch angles. . . . . . . . . . . . . . .

5.17 Blade power (a) and thrust (b) coefficients normalised with respect to the integrated rotor force coefficients for three different blade pitch angles during one rotation cycle. . . . . . . . . . . . . .

5.18 Spanwise variation (rotor swept area) of approach angle, $\phi^{\circ}$, on a turbine blade for three different blade pitch angles, (a) $0^{\circ}$, (b) $10^{\circ}$ and (c) $20^{\circ}$ during a rotation cycle. . . . . . . . . . . . . .

5.19 Spanwise variation (rotor swept area) of angle of attack, $\alpha^{\circ}$, on a turbine blade for three different blade pitch angles, (a) $0^{\circ}$, (b) $10^{\circ}$ and (c) $20^{\circ}$ during a rotation cycle. . . . . . . . . . . . . . .

5.20 Angle of attack variation along the span of a turbine blade for three different blade pitch angles at the top (solid) and bottom (dashed) position in a rotation cycle. . . . . . . . . . . . . . . . . . . 14 
5.21 Sectional plots of pressure distribution on the blade at $180^{\circ}$ position (bottom rotational position) at (a) $r / R=0.5$ and (b) $r / R=0.95$ to observe the location of stagnation point. Stagnation point moves from the lower surface to upper surface of the aerofoil indicating a negative angle of attack towards the tip of the blade. . . . . . . . . . . . . . .

5.22 Spanwise axial force, in N, variation on a blade for three different blade pitch angles, (a) $0^{\circ}$, (b) $10^{\circ}$ and (c) $20^{\circ}$ in shear flow. Notice that the contour range is different in each case. . . . . . . . . . . . . .

5.23 Sectional force variation along the span of a turbine blade for three different blade pitch angles, $0^{\circ}, 10^{\circ}$ and $20^{\circ}$, at the top and bottom position in a rotation cycle. . . . . . . . . . . . . . . . . . . . . .

5.24 Vertical variation in normalised velocity deficit at four different streamwise locations, $0.125 \mathrm{D}, 0.5 \mathrm{D}, 1 \mathrm{D}$ and $2 \mathrm{D}$ in the near wake for three different blade pitch angles, $0^{\circ}, 10^{\circ}$ and $20^{\circ}$. The velocities are normalised with respect to the inlet shear profile. $0.125 \mathrm{D}$ is the location of a potential support structure which will be discussed in the next chapter. . . . . . .

5.25 Variation in normalised axial velocity at lateral mid-section for two different blade pitch angles, $0^{\circ}$ and $10^{\circ}$. The velocities are normalised with depth averaged velocity. . . . . . . . . . . . . .

5.26 Cross-stream variation in turbulence intensity (TI) at three different streamwise locations, (a) $0.5 \mathrm{D}$, (b) $1 \mathrm{D}$ and (c) $2 \mathrm{D}$ in the wake downstream for $0^{\circ}$ pitch (left) and $10^{\circ}$ pitch (right) cases. . . . . . . . . . . . .

5.27 $\mathrm{Q}$ criterion for two different blade pitch angles, (a) $0^{\circ}$ and (b) $10^{\circ}$ in shear flow; $\mathrm{Q}=0.2 \ldots \ldots \ldots \ldots \ldots$

6.1 Dimensions of the computational domain. The flow direction is in the

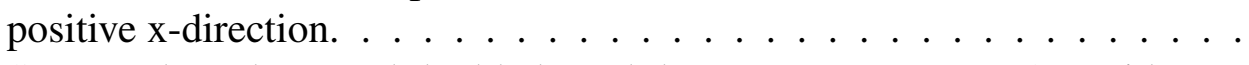

6.2 Structural mesh around the blade and the support structure. $\mathrm{O}$ - grid was used around the support structure in the outer domain. . . . . . . . . . . . 158

6.3 Vertical profile of mean axial velocity through the rotor normalized with the depth-averaged bulk flow velocity. . . . . . . . . . . . . . . . . . 160

6.4 Blade power (a) and thrust (b) coefficients normalised with respect to the rotor force coefficients for different support structures, cylinder and ellipse, at $10^{\circ}$ blade pitch angle during one rotation cycle. . . . . . . . . 161

6.5 Spanwise variation of angle of attack, $\alpha^{\circ}$, on a turbine blade for different support structures at $10^{\circ}$ blade pitch angle during a rotation cycle. . . . . 163

6.6 Angle of attack variation along the span of a turbine blade in the presence of different support structures for $10^{\circ}$ blade pitch angle, at the top (solid line) and bottom (dashed line) position in a rotation cycle. . . . . . . .

6.7 Spanwise axial force $\left(\mathrm{N} \mathrm{m}^{-1}\right)$ variation on a blade for different support structures, cylinder and ellipse, in shear flow. . . . . . . . . . . 165 
6.8 Sectional force variation along the span of a turbine blade for different support structures, cylinder and ellipse, at the top and bottom position in a rotation cycle. $\ldots \ldots \ldots \ldots \ldots$

6.9 Stagnation line on the frontal area of the cylindrical support structure. $\mathrm{D}_{S}$ represents the diameter of the cylinder and y represents the variation along the diameter in the direction transverse to the flow. The dashed line indicates the stagnation line in the absence of a rotor. The deviation can be observed behind the rotor. . . . . . . . . . . . . . . . . . . . . 168

6.10 Stream-wise sectional forces on support structures, cylinder (a) and ellipse (b) during one rotation cycle. . . . . . . . . . . . . . 166

6.11 Pressure slices at $t / T=0.15$. The leading edge of the blade section at $\mathrm{z} / \mathrm{R}$ $=-0.4$ (a) has just passed the centre line of the support structure while the blade section at $\mathrm{z} / \mathrm{R}=-0.8$ (b) is just approaching the centre line. . . . . .

6.12 Cross-stream sectional forces on support structures, circular (a) and ellipse (b) during one rotation cycle. . . . . . . . . . . . . .

6.13 Mean sectional force variation along the support structure height, $\mathrm{L} / \mathrm{D}=0$ at the hub and $\mathrm{L} / \mathrm{D}=1$ at the seabed. $\mathrm{F}_{x}$ is in the direction of the flow and $\mathrm{F}_{y}$ being in the cross-stream direction.

6.14 Vertical variation in normalised velocity deficit at three different streamwise locations, $\mathrm{x} / \mathrm{D}=0.125,1$ and 2 in the near wake for the $10^{\circ}$ blade pitch angle. The velocities are normalised with respect to the inlet shear profile. $0.125 \mathrm{D}$ is the location just ahead of the support structure. . . . . .

6.15 Variation in normalised axial velocity at lateral mid-section for different support structures, cylinder and ellipse. The velocities are normalised with depth-averaged velocity. . . . . . . . . . . . . .

7.1 Time-averaged total farm power output, $\left\langle P_{f}>\right.$, as a function of the number of turbines, n, installed and support structure drag coefficient. $\left(\chi=0.16, F r_{w}=0.478, L / h=125, C_{f}=0.002\right) \ldots \ldots \ldots$

7.2 Channel power coefficient, $C_{P c}$, as a function of channel thrust coefficient, $C_{T c}$, for (a) $F r_{w}=0.673(a=0.125 m)$, (b) $F r_{w}=0.478(a=0.25 m)$, (c) $F r_{w}=0.338(a=0.5 m)$. The dashed lines indicate constraints on change in the maximum volume flow rate in the channel. $(L / h=$ $\left.125, C_{f}=0.002, \chi=0.16\right) . \ldots \ldots \ldots \ldots$

7.3 Channel power coefficient, $C_{P c}$, as a function of channel thrust coefficient, $C_{T c}$, for (a) $L / h=75$, (b) $L / h=125$, (c) $L / h=175$. The dashed lines indicate constraints on change in the maximum volume flow rate in the channel $\left(F r_{w}=0.478, C_{f}=0.002, \chi=0.16\right)$. Note that figure $7.2 \mathrm{~b}$ is the same as $7.3 \mathrm{~b}$ because they have the same $F r_{w}$ and $L / h \ldots \ldots$. . .

7.4 Maximum undisturbed channel velocity (due to different $F r_{w}$ ) vs. the time averaged power output of the tidal farm, $\left\langle P_{f}\right\rangle$, for different support structure drag coefficients. $\left(L / h=125, C_{f}=0.002, \chi=0.16\right) . \ldots 188$ 
7.5 Maximum undisturbed channel velocity (due to different $L / h$ ) vs. the time averaged power output of the tidal farm, $\left\langle P_{f}\right\rangle$, for different support structure drag coefficients. $\left(F r_{w}=0.478, C_{f}=0.002, \chi=0.16\right) . \quad \ldots 188$ 


\section{Nomenclature}

$<P_{f}>$ Time-averaged farm power output

$\chi \quad$ Ratio of support structure frontal area to turbine rotor swept area

$\Delta t \quad$ Time step

$\eta \quad$ Turbine efficiency

$\omega \quad$ Frequency of the tide

$\rho \quad$ Density of fluid

$\xi \quad$ Ratio of total rotor frontal area to channel cross-sectional area

$\zeta_{0} \quad$ Head difference along the channel

$A_{b} \quad$ Seabed area

$a_{i} \quad$ Axial induction factor

$A_{s} \quad$ Frontal area of the support structure

$A_{t} \quad$ Turbine rotor swept area

$C_{D} \quad$ Support structure drag coefficient

$C_{f} \quad$ Seabed friction coefficient 
$C_{L} \quad$ Lift coefficient

$C_{P} \quad$ Turbine power coefficient

$C_{T} \quad$ Turbine rotor thrust coefficient

$C_{P c} \quad$ Channel power coefficient

$C_{T c} \quad$ Channel thrust coefficient

$C_{T T} \quad$ Total turbine thrust coefficient

$F_{S} \quad$ Drag on the support structure

$F r_{w} \quad$ Similar to Froude number, $\omega L / \sqrt{g a}$

$g \quad$ Acceleration due to gravity, $\mathrm{ms}^{-2}$

$h \quad$ Depth of the channel

$L \quad$ Length of the channel

$Q \quad$ Channel volume flow rate

$Q_{0} \quad$ Magnitude of the volume flux in the absence of flow resistance

$T \quad$ Turbine thrust

$U_{A} \quad$ Channel velocity 


\section{Chapter 1}

\section{Introduction}

Tidal energy is one of the most exciting emerging forms of renewable energy, especially when the UK government is committed to reducing the greenhouse gas emissions by $80 \%$ by 2050 and to meeting $15 \%$ of the energy demands from renewable sources by 2020 (Severn Tidal Power, 2010).

The UK has one of the largest tidal energy resources in the world. The total tidal power estimation from all the UK sites is around $30 \mathrm{TWh} / \mathrm{year}$ with the highest resource at Pentland Firth Deep, which alone can contribute up to $10 \mathrm{TWh} / \mathrm{year}$ (Carbon Trust, 2011).

In addition to helping reduce carbon emissions, the tidal energy sector can bring significant economic benefits. Tidal energy has the potential to power 15 million homes, save 70 million tonnes of carbon and create 16,000 jobs in the UK alone i.e. tidal power could provide up to $15 \%$ of the current electricity generation from an indigenous renewable source, and bring new employment opportunity both locally and nationally (Marine Current Turbines, 2011).

The last few years have seen an immense amount of activity in this sector. There have been more than 12 large-scale prototype devices deployed or installed around the UK and 
seabed leases awarded for over $1.8 G W$ of power production, including the first Northern Ireland leasing round. Major engineering firms such as Siemens, Andritz, Voith, Alstom and $\mathrm{ABB}$ are working together with major utilities such as SSE, E.ON, RWE Innogy, Vattenfall, EDF and ScottishPower Renewables to take the industry forward (RenewableUK, 2013).

Between 2010 and 2012, The Crown Estate licensed almost 40 wave and tidal sites throughout the UK. While the vast majority are in Scottish waters, sites are also being developed as far and wide as Falmouth in the south west of England and Torr Head in Northern Ireland. These sites mean that there is now a substantial pipeline of potential capacity, with likely deployment of 100 to $200 \mathrm{MW}$ of devices expected by the end of 2020 (RenewableUK, 2013).

Although different design configurations of tidal turbines have been proposed by various researchers, it is commonly agreed that a large number of turbines need to be installed in a candidate channel for tidal power generation to be viable. A number of issues need to be addressed before installing a large number of turbines in the channel, amongst them: i) The large opposing thrust presented to the channel due to the presence of these turbines which can reduce farm power potential and ii) The influence of these turbines on the environment particularly due to channel flow rate reduction.

The aim of this project is to reduce the drag force on the turbine system, or more precisely the support structure thrust per MW of power generated, which would reduce the opposing thrust presented to the channel. This would result in an increased productivity from the turbine farm. The most common configuration of a turbine is to install them on the seabed by means of a support structure which is usually in cylindrical shape. These support structures can experience a significant drag force and hence predicting and designing for these forces is of great importance to the integrity of any turbine installation. It is 
particularly important for the support structures of tidal turbines as the force on the support structure acts to parasitically reduce the power available to the turbines. This project will examine the hydrodynamics of bluff support structures for tidal turbines with the goal of minimizing the forces on them, over a range of operating points. The research objectives are discussed in detail in the next section.

This chapter is structured such that a basic introduction to tides and tidal energy extraction is given in the first section. The main research objectives of this thesis are discussed in the second section. The next two sections discuss the literature on modelling tidal turbine farms and turbine rotors in detail. The next section discusses about the environmental conditions in a tidal channel such as the turbulence and the shear velocity profile, and the numerical modelling of these conditions. Later, the different support structure shapes and their influence on the turbine system is discussed in detail by presenting the previous work in this area. The last section outlines all the chapters of this thesis.

\subsection{Tides and tidal energy extraction}

In simple terms, tides are the rise and fall of sea levels due to the gravitational force between the Earth and the Sun, and the Earth and the Moon. The complete theory behind the tides is more complex.

The study of tides has been a fascinating concept for the scientific community since ancient Greece to the modern era with Galileo, Newton, Euler, Laplace and many others trying to explain the physics behind the tides (Deparis et al., 2013). Laplace (1782) developed a dynamic theory of tides which was a landmark study in understanding the tides. By neglecting the vertical velocity, Laplace established an analytical model, which remains the basis of tidal flow theory.

As the Earth revolves around the Sun, the gravitational force $\left(\mathrm{F}_{2}\right)$ is experienced by the 


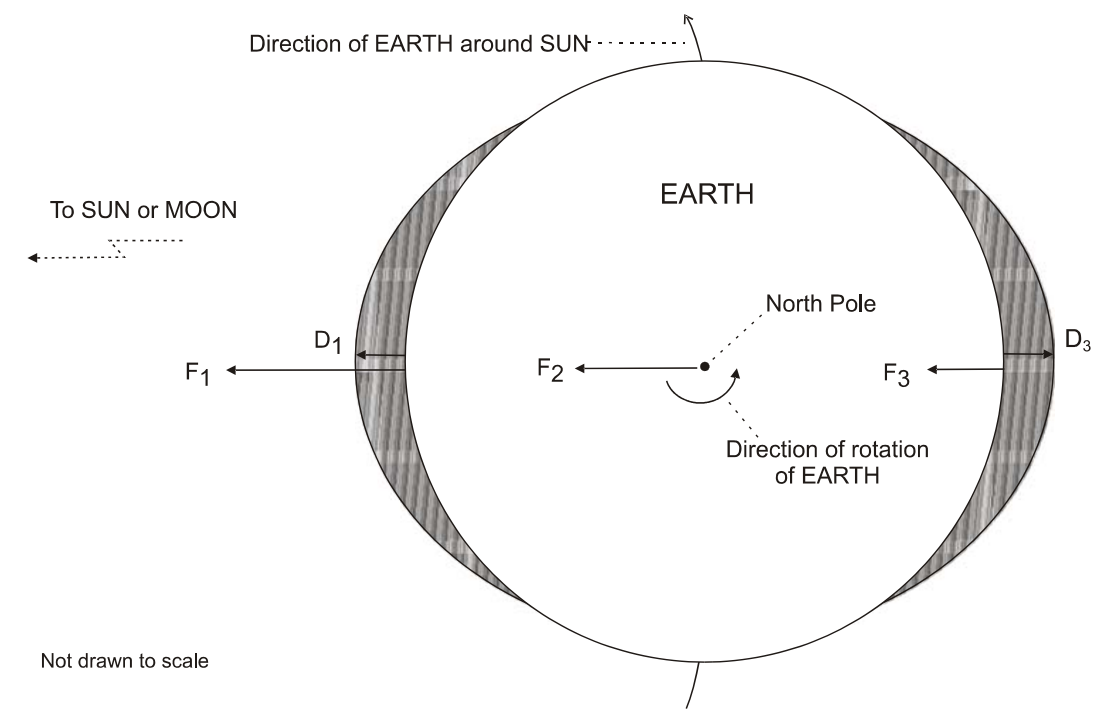

Figure 1.1: Tides generated due to gravitational forces involving sun, moon and earth (Hicks, 2006)

Earth, acting towards the Sun; see figure 1.1. This force is higher at the point on Earth's surface closest to the Sun and lower at the point farthest away from the Sun, $F_{1}>F_{2}>F_{3}$. The tide generating forces are the differences in the gravitational forces at the centre of the Earth and the point of interest (Hicks, 2006). $D_{1}=F_{1}-F_{2}$ is the force directed towards the Sun and $D_{3}=F_{2}-F_{3}$ is the force directed away from the Sun. This causes a bulge in the water distribution which is further affected by the presence of continents, turbulence, viscosity and many other factors.

A similar phenomenon occurs due to the gravitational force between the Earth and the Moon. Although the Moon is much smaller than the Sun, it is much closer to the Earth than the Sun and the lunar tidal forces are infact stronger than those due to the Sun; the tides due to the Sun are approximately $27 / 59$ of the strength of the lunar tides (Komar, 1998).

Each of the tide generating motions can be represented by a simple harmonic cosine curve which is known as a tidal constituent. This is useful in studying the influence due to a 
particular tidal generating mechanism or a combination of few of them. A tidal constituent is represented by a letter followed by a subscript such as $\mathrm{S}_{2}$. S indicates that this is due to the Sun and the subscript '2' states that there are two tidal cycles for each astronomical cycle. $\mathrm{S}_{2}$ is called the Principal Solar semi-diurnal constituent with a time period of 12 hours. Similarly $\mathrm{M}_{2}$ is called the Principal Lunar semi-diurnal constituent with a time period of 12.42 hours.

There are a large number of tidal constituents but only few of them would be of particular importance depending on the tidal site under investigation. Some of the other important tidal constituents include the Larger Lunar Elliptic semi-diurnal constituent, $\mathrm{N}_{2}$, the Smaller Lunar Elliptic semi-diurnal constituent, $\mathrm{L}_{2}$, Principal Lunar Declinational diurnal constituent, $\mathrm{O}_{1}$, Principal Solar Declinational diurnal constituent, $\mathrm{P}_{1}$ among others. $\mathrm{M}_{2}$ is usually the most dominant constituent and hence, it is the only one considered in this thesis.

The free flow of the tides around the globe is obstructed by the presence of the continents. The tides and tidal elevation is hence a result of the interaction between the tides generated in the deep ocean and the continental shelf. The higher tidal elevations are observed at the coast are centred on regions of smaller tidal range, amphidromic points. The tidal amplitude near the coast may become larger either due to the narrowing of the channel, thus increasing the energy flux, or due to the constructive interference between incoming and reflected components of the tide (Plumb and Marshall, 2007). Also the presence of continents results in different responses to tide generating forces as each basin has its own mode of oscillation. The Coriolis acceleration due to the rotation of the Earth also influences the wave propagation (Pugh, 2004)

Tidal energy extraction has gained particular importance in the recent years with the world moving towards renewable energy. Tidal energy extraction devices can be broadly 


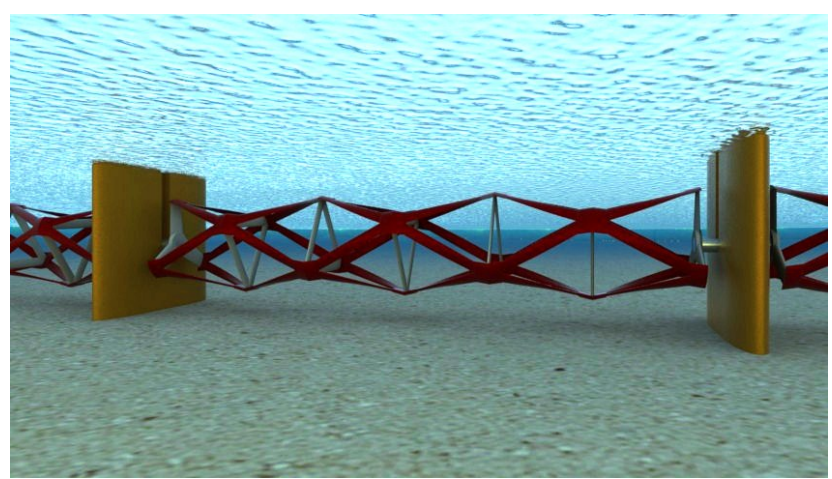

(a) Transverse Horizontal Axis Water Turbine (THAWT) $^{1}$

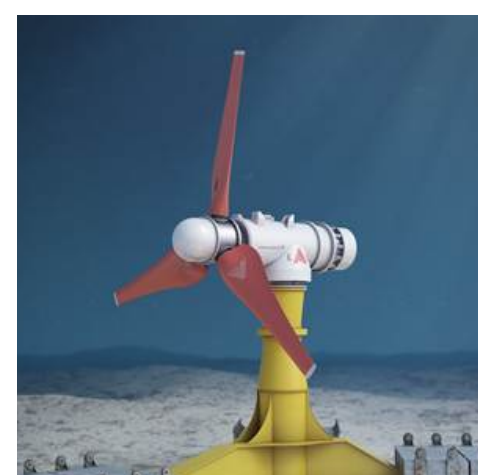

(b) Horizontal Axis Water Turbine $\left(\mathrm{HAWT}^{2}\right.$

Figure 1.2: Types of tidal current turbines

classified into two types - tidal barrages and tidal current turbines. In a tidal barrage, an estuary or a bay is enclosed by a barrage or dam, letting the water in during the high tide and later using its potential energy to generate electricity.

The tidal current turbines can further be classified into two main types - Transverse Horizontal Axis Water Turbine (THAWT) and Horizontal Axis Water Turbine (HAWT); see figure 1.2. The motivation behind the THAWT models is to maximise the crosssectional area occupied by the turbine in the channel which would result in larger power output. But these devices might increase the opposing drag in the channel which would reduce the flow velocity and hence reduce the basin efficiency; basin efficiency is defined as the ratio of power generated in a channel to the power removed from the channel which is due to the opposing thrust presented by the turbines. HAWT are similar to wind turbine rotors mounted either on monopiles or different support structure mechanisms. Most of the tidal turbine devices under consideration for deployment are horizontal axis type turbines.

There are a few other types of turbines in conceptual stage such as ducted turbines, oscillating hydrofoils and tidal kites. In ducted flow turbines, ducts or diffusers are added

\footnotetext{
${ }^{1}$ http://www.keplerenergy.co.uk

${ }^{2} \mathrm{http}: / / \mathrm{www}$.windpowerengineering.com
} 
around the turbine rotors to increase the mass flow rate around the rotor and hence an increased power output. Although the ducted turbines may increase the power output, the higher drag on the turbine system due to the presence of ducts reduces the overall efficiency (Fleming, 2014).

An oscillating hydrofoil consists of a hydrofoil which is free to oscillate the vertical direction due to the lift generated by the tidal currents. The hydrofoil is fixed to a lever which allows it to oscillate freely in the vertical direction and the lever itself is connected to the seabed. A tidal kite is a small turbine with a hydrofoil wing at the top and a rudder at the back, and the whole set-up is tethered to the seabed. Using the tension in the tethered string and the rudder, the kite can be directed to move along a given trajectory through the water column. The mechanical energy of the turbine is then converted to electrical energy for power output (Roberts et al., 2016). There are also some designs for plausible floating tidal turbines (Elghali et al., 2007) but most of them are still in the conceptual stage and hence this thesis is confined to horizontal axis tidal turbines.

\subsection{Modelling of tidal farms}

Lanchester (1915) and Betz (1920) were the pioneers in the field of wind turbine aerodynamics and they presented (independently) an analytical model for a wind turbine in a flow, to estimate the maximum power that can be generated independent of the turbine design, which is now commonly known as the Betz limit. This model assumes a turbine is an idealized actuator disc which extracts energy from the incoming flow. The model is suitable for a single turbine in an unconstrained flow but in the presence of a large number of turbines in a channel, new models have been and continue to be developed.

Various analytical methods have been proposed to model the turbine arrays in a channel in the recent past. Most of these models are inviscid models because the key processes 
in the flow are primarily inviscid mechanisms. The analytical methods have many other simplifying assumptions but are useful in providing an estimate of the power potential of a tidal channel at very low computational costs.

It was initially considered that the kinetic energy flux in a channel is a measure of the power potential but Garrett and Cummins (2005) have shown that this assumption is incorrect. The most important distinction in this model from previous models is that the volume flow rate in the channel was considered a variable which would change as the opposing thrust presented to the channel is changed. The power was calculated as a product of the opposing force due to turbines in the channel and the volume flux through the channel (This has to be multiplied by the turbine efficiency to get the average electrical power output). It was also discussed that as opposing force in the channel increases, there is a reduction in the channel flow rate which indicates a presence of peak in the power output as shown in the paper. This opposing force may be due to bed friction as well as turbine drag and in the case of turbine drag, it indicates that installing too many turbines reduces the power output and hence an optimum number of turbines need to be installed to achieve maximum obtainable power. Garrett and Cummins (2005) postulated that the maximum average power in a channel is between $20 \%$ and $24 \%$ of the product of the peak tidal head between the ends of the channel, and the peak undisturbed mass flux through the channel, depending on whether the flow is dominated by bed friction or no bed friction.

The impact of support structures was not mentioned in Garrett and Cummins (2005) but Garrett and Cummins (2008) discussed that the support structures play a significant role in reducing the optimum number of turbines required for maximum obtainable power. According to them, in the presence of support structures, the maximum power estimates must be reduced by a factor of $F_{t}\left(F_{S}+F_{t}\right)^{-1}$ where $F_{t}$ and $F_{S}$ are drag on turbine rotor and support structure respectively. This is because the drag on the support structure 
reduces the flow rate without generating any power. It also discusses about the reduction in flow rate due to increasing opposing drag and mentions that if the flow rate reduction is limited to $10 \%$, the power coefficient drops by more than $50 \%$. This is an important observation considering that there might be a constraint on reduction in channel flow rate for environmental reasons.

Vennell (2010) extended the work of Garrett and Cummins (2005) to show that the internal configuration of a turbine farm, such as the number of turbines per row, number of rows affect the power production. The analysis is based on 1D shallow water momentum balance equations wherein the opposing forces are due to the channel bed friction and turbines in the farm. This work was extended in Vennell (2012) which includes the impact of support structures. In this work, the total power lost to drag was divided into three parts, namely power lost to background friction, power used for electricity production and power lost to drag on turbine support structures. It discusses about how the support structure drag limits the maximum number of turbines installed in the channel for optimum power output.

Smeaton et al. (2016) used a 1D model to study the power output from tidal channels with narrow constrictions which have energy dense, high velocity flows due to the constrictions. It was shown that the presence of turbines reduces the flow velocity and the reduction in flow velocity needed to achieve the peak farm power was independent of channel constriction. Also an approximate estimate of power potential in a constricted channel was presented based on the head difference across the channel and the flow velocity.

Adcock et al. (2013) have used a depth-averaged numerical model to estimate the power output from Pentland Firth. They comment on an upper limit on the number of rows of turbines that can be installed in a channel due to diminishing returns on power if too many turbines are installed. Another important aspect of their discussion was the 
impact of installing turbines on the environment, particularly channel flow rate, and they report that to obtain the upper bound on power extraction requires a $30 \%$ reduction in the tidal current velocity. The influence of bed friction coefficient was also considered and the role it plays in decreasing the farm power output was discussed.

Funke et al. (2016) modelled the tidal turbine farm as a mathematical optimisation problem constrained by the depth-averaged shallow water equations. In this model, the turbine farm is represented by a turbine density function which defines the number of turbines per unit area and it is optimised for maximum economic profit. Murray and Gallego (2017) used a three-dimensional hydrodynamic ocean model to investigate the tidal stream resource and its impact on the environment in Pentland Firth. Their upper bound estimates of power output were approximately 25\%-30\% higher than the previous studies involving 2D models. Although the 2D and 3D farm models are useful to estimate the tidal resource for site specific investigations, since the channel considered in this thesis was a generalised channel, a simpler 1D model was used which will be introduced in Chapter 2, also leading to lower computational cost.

The work discussed so far has presented various models of flow past turbine arrays and indicated the significance of support structures but none have extensively considered the impact of various support structure shapes and the environmental impact (constraint on flow rate reduction). This thesis investigates the impact of various support structure shapes on the farm power output along with the volume flow rate constraint. 


\subsection{Numerical modelling of rotors}

\subsubsection{Actuator Disc Model}

An actuator line model is an analytical model and is one of the earliest representation of turbine or propeller. Lanchester (1915), Betz (1920) and Joukowsky (1920) have independently developed this analytical model to simulate a turbine in an unconstrained flow (van Kuik, 2007).

An actuator Disc Model involves the analysis of a turbine without particular specification of the turbine blades. This is a basic model in which the turbine is replaced by a circular disc and a pressure difference is applied across the disc. This model can provide a reasonable estimate of the efficiency of the turbine and is usually used for design of large array of turbines to reduce the computational costs. This model cannot particularly predict the wake pattern and the swirl behind a rotor because there are no data on actual turbine blades involved in the model.

This model is physically analogous to a porous disc which is used in the model-scale experiments on tidal arrays as discussed in Graham (1976), Draper et al. (2013). Whelan et al. (2009) used the actuator disc model to study the free surface effects and blockage effects in the presence of a tidal turbine array and compared them with experimental results with good accuracy in specific cases. Hence these models are a quick approximation with minimal computational cost.

In the recent past, advanced models based on actuator disc theory have been proposed such as Shives and Crawford (2017), wherein a computational efficient CFD method was used along with actuator disc theory to predict the wake interaction and blockage effects. This method accurately predicted the rotor performance of turbines located in wake of other turbines. The velocity in the far wake is reasonably predicted using this method, but 
it fails to predict the near wake velocity which is needed to study the rotor-support structure interaction. Hence this method would be a computationally efficient way to model large tidal arrays but is not fit to simulate complex device-scale interaction problems.

It is also a steady state model which makes it unsuitable to study the interaction effects of a turbine and its support structure which is inherently unsteady.

\subsubsection{RANS-BEM}

In the Blade Element Momentum (BEM) model, the turbine blade is analyzed as a number of independent elements along the span-wise direction. The velocity induced at each element is obtained by performing the momentum balance for an annular control volume containing the blade element. Then the aerodynamic lift and drag forces on each element are calculated using the coefficients from the empirical two-dimensional wind tunnel test data at the geometric angle of attack of the blade element relative to the local flow velocity (Kulunk, 2011).

CFD models such as Reynolds averaged Navier Stokes (RANS) model have been used in the past to simulate the flow past the turbines, using BEM to obtain the impact forces of turbine on the flow. This way the turbine itself need not be computationally modelled which would lead to high computational costs.

The RANS-BEM approach was originally applied to wind turbines (Ammara et al., 2002) which was later adopted to tidal turbine simulations. Turnock et al. (2011) demonstrated that the rotor-generated turbulence and swirl can have a big impact on wake evolution by coupling the BEM method with the RANS model. Belloni (2013) used the RANS-BEM approach to perform a computationally efficient design study of ducted and open-centre turbines. Schluntz and Willden (2015a) used the RANS-BEM approach to study the effect of blockage on tidal turbine performance by varying the lateral spacing of 
turbine rotors.

Olczak et al. (2016) have implemented a RANS-BEM model in the commercial software STARCCM+ to model tidal turbine arrays, and compared the wake velocity and rotor thrust with laboratory measurements. The basic RANS-BEM model predicted reasonably the wake velocity behind a single rotor at distances greater than $4 \mathrm{D}$, and between $0.5 \mathrm{D}$ and 4D, the wake velocity was reasonably predicted in the outer half of the rotor. The model was further developed by including the turbulent kinetic energy generated by the tip which was predicted by the blade resolved RANS CFD. This enhanced model improved the prediction of wake velocity and wake rotation within 4D downstream. The average thrust on a rotor in the first row of rotors in the array has been predicted within $10 \%$ accuracy which is a reasonable trade-off for the lower computational cost.

Hence these models have been known to reasonably predict the wake of the turbines including swirl effects but these studies were done considering only the turbine rotor without the support structures. This model is a steady state model and when the support structures are included behind the turbines, the flow past the bluff support structures which is inherently unsteady will not be captured by this model. The lower computational cost of this model makes it suitable to simulate large arrays of turbines with reasonable accuracy but may not be applicable at device scale to study the interaction between the rotor and its support structure.

\subsubsection{Actuator Line Model}

To overcome the limitations of the steady state models, an unsteady model, the actuator line model was developed by Sorensen and Shen (2002) for simulating wind turbines. In this method, the flow is modelled by using 3D Navier-Stokes equations with the turbines being replaced by actuator lines. These actuator lines are supplied with the airfoil data 
to represent the loading on each blade. Hence the turbine blades are not computationally modelled but instead their influence on the flow field is obtained as integrated quantities from the airfoil data. An iterative process is used to determine the subsequent loading by computing local angles of attack from the blade movement and local flow field. Hence the flow characteristics including the unsteady wake can be modelled at reasonable computational costs.

Another advantage of the actuator line model is that the actuator lines can rotate in a fixed plane that can be coned, yawed or tilted in any direction relative to the inflow direction (Mikkelsen (2003)).

Sorensen and Shen (2002) have performed simulations on wind turbines and compared their predictions with experimental data, and reported reasonably accurate results but the results are not consistent across different turbine geometries.

The Sorensen and Shen (2002) model was applied to tidal turbines by Churchfield et al. (2013) using Large Eddy Simulation (LES) to resolve the flow field. The forces on the rotor were consistent but the authors suggest an improvement in the model to accurately capture the wake of the rotor.

A few other models have been developed on similar lines, one such method being used by Schluntz and Willden (2015b) wherein a new velocity sampling technique was used to bypass the requirement of smearing techniques as in other actuator line models. The forces on the rotor were predicted to good accuracy but there was still some discrepancy with the experimental results. Also the authors suggest improving the spanwise force coefficients with the addition of a stall-delay model.

Wimshurst and Willden (2016) have simulated an actuator line model using the data from polars extracted from a fully resolved steady simulation. This method showed an improvement in the results over the simulation performed using $2 \mathrm{D}$ aerodynamic data. 
The overall force coefficients from the actuator line method were in reasonable agreement with the fully resolved blade model but the comparison of blade inboard sections were found to show discrepancies.

The previous work suggests that the actuator line model might be a good approximation to calculate the turbine forces, saving considerable time, but it may not be reliable in analysing the wake and thus the interaction effects between the rotor and its support structure.

\subsubsection{Blade resolved model}

To model the interaction between the rotor and the support structure accurately, it is necessary to fully resolve the turbine blades using a fine grid around the blade as the models discussed earlier such as the BEM-RANS, actuator line are based on the lift and drag measurements of two-dimensional aerofoils and the 3D effects are not completely captured. The blade-resolved models are computationally expensive due to the high velocity gradients around the blades and to resolve them requires a fine mesh.

The blade-resolved model can be applied in two different strategies - Multiple Reference Frame (MRF) model and mesh rotation model. In the MRF model, the mesh around the rotor remains stationary but the flow equations are solved in a rotating reference frame, thus simulating rotor rotation, leading to lower computation time. In the mesh rotation model, the mesh is physically rotated around the rotor which results in higher computational cost. Both these strategies are employed in this thesis.

The MRF model was originally applied to wind turbines by Sorensen et al. (2002) to predict the turbine performance at a range of inlet velocities. It was observed that the rotor torque and thrust were in good agreement until pre-stall conditions using this model. The same approach was later used by Hu et al. (2006) to study stall delay, and by Ferrer and 
Munduate (2007) to study the effect of blade tip geometry on turbine performance.

O'Doherty et al. (2009) adopted the MRF model to simulate tidal turbines using different turbulence models to predict the power and thrust coefficients of a rotor. The MRF model provides a good approximation to simulate steady state flows in a uniform flow but in a shear flow, the MRF model does not apply because the blades are stationary and each blade would experience only a particular portion of the flow. Hence a mesh rotation model is used to simulate unsteady shear flows.

McSherry et al. (2011) employed the unsteady mesh rotation strategy to simulate a model turbine to compare with the experiments of Bahaj et al. (2007b). It was reported that the power and thrust coefficient were in good agreement with experimental results at lower tip speed ratios and the discrepancies at higher tip speed ratios was attributed to the occurrence of cavitation.

Afgan et al. (2013) simulated a tidal turbine using the RANS model and compared the influence of two different turbulence models, k- $\omega$ SST and Reynolds Stress Model, on the prediction of power and thrust coefficients. Later, LES simulation was also carried out on the same turbine and it was reported that LES resolves the blade tip vortices better than the RANS model but has a much higher computational cost. Also it was observed that the nacelle and the support structure have a strong effect on the wake behaviour.

Mason-Jones et al. (2013) have shown the influence of shear profile and support structures on a tidal turbine using a fully resolved blade model. Since it was a fully resolved model, it was possible to study the interaction effects between the rotor and the support structure, and the variation in force coefficients as a blade completes one rotation is presented to demonstrate this.

McNaughton (2013) implemented a new sliding mesh method with a fully bladeresolved turbine inside the sliding mesh. The method was validated by simulating the 
experiments of Bahaj et al. (2007b), which were shown to be in good agreement. Later a full-scale rotor was simulated in a sheared flow using the fully resolved blade model to study the influence of shear on the turbine characteristics. Along with shear, the influence of free-stream turbulence and yaw in the flow on turbine performance was also discussed.

Ahmed et al. (2017) simulated a full scale tidal turbine installed at the EMEC site, and compared the fluctuating power and thrust coefficients with real site data. Both RANS and LES simulations were performed with different inflow turbulence parameters. It was reported that at lower turbulence levels, both RANS and LES predict similar blade pressures and phase-averaged loads. Hence the RANS model would be sufficient to determine the mean loads in this case. It was shown that LES resolves blade-generated turbulence as well but it only contributes a small fraction of the overall load fluctuation. Since the RANS model is computationally less intensive but still able to predict the flow features under most operating conditions, it is used in the current thesis.

\subsection{Free stream turbulence and shear flow}

There have been numerous studies on turbulence and shear flow in a tidal channel in the past. Lu and Lueck (1999b) have discussed the effects of shear velocity profile through ADCP measurements that reveal secondary circulation, shear reversals and transverse velocity shear in a tidal flow. In a further paper, $\mathrm{Lu}$ and Lueck (1999a) analyze the turbulence in the channel by using ADCP measurements to calculate the turbulent kinetic energy and vertical eddy viscosity in the channel.

Ralston and Stacey (2006) discuss the complexity of shear and turbulence in a tidal flow which may vary due to bathymetry, flow angle and the strength of the longitudinal salinity gradient. They report that the turbulence which is produced closer to the channel banks locally exceeds dissipation, and any excess energy either diffuses vertically or is 
lost to buoyancy.

Mathis et al. (2013) developed a model for predicting the fluctuations of the wall shearstress in turbulent boundary layers and applied it to tidal flows in Mathis et al. (2014). This model was validated with the experimental data from a shallow tidal channel at Suisun Slough in North San Francisco Bay and reasonably predicted the correct spectral content and the probability density function. But there was a large disparity in the prediction of the bed shear stress compared to the conventional methods. Since there is no concrete method to predict the bed shear-stress, several assumptions had to be made to simulate the shear in the flow and the bed shear-stress. Gunn and Stock-Williams (2013) present a comprehensive study of hydrodynamic models to simulate tidal flow which indicates the complexity of the shear flow and the free stream turbulence.

The majority of tidal turbine device scale simulations in the past have been performed in a uniform flow but in the recent past, the importance of shear flow on tidal turbine performance has been discussed ( Fleming (2014), McNaughton (2013)). In models such as Mason-Jones et al. (2013), the inlet velocity was simplified based on $1 / 7^{\text {th }}$ power law whereas more advanced methods were used in Fleming (2014) and McNaughton (2013) to specify the inlet conditions for shear and turbulence. McNaughton (2013) used real site data to generate polynomial profiles for inlet velocity but the shear profile at the inlet was not sustained by the time it reached the turbine rotor plane. Batten et al. (2013) tried to overcome this issue by specifying high turbulence at the inlet but McNaughton (2013) showed that this can alter the shear velocity profile by the time it reaches the rotor plane.

Fleming (2014) developed a model wherein along with the shear velocity profile at the inlet, a turbulence inlet profile and wall shear-stress at the bed are also specified and it was observed that the shear velocity profile was maintained by the time the flow reaches the rotor plane. This approach was used in the current work to simulate the shear flow. 
A different approach was proposed by Gant and Stallard (2008) wherein an unsteady inlet boundary condition was specified to generate large-scale structures in the flow past a porous disc. This resulted in a turbulent flow which sustained the shear profile well downstream. It was reported that the wake mixing behind the rotor was also enhanced compared to a steady state inflow condition. Although it models the shear flow accurately, due to the already high computational cost associated with simulating the rotor and its support structure in the current work, this method was not considered further herein.

\subsection{Support structures}

In this section, different support structure shapes and their effects on turbine systems are discussed. Orme and Masters (2006) presented the different types of potential support structures which can be used for horizontal axis tidal turbines. This classification was based on functional specification, profitability and cost estimations. Among these there are two turbine systems which are currently used in many designs. These are the sheath type and top-mounted type; see figure 1.3.

In the sheath type support structure, the support structure is protruding through the water surface so that the position of the tidal turbine rotor can be varied along the channel depth. This can also facilitate in easier maintenance since its easier to access the rotor. But the presence of the tower above the water surface can cause an issue with the ships travelling in that region and hence may not be possible in all tidal channels. The top-mounted monopile structures are below the water level and there is enough clearance between the tip of the blade and the channel surface so as to prevent disruption to navigation in the channel. The scope of this thesis is limited to these top-mounted monopile structures. 


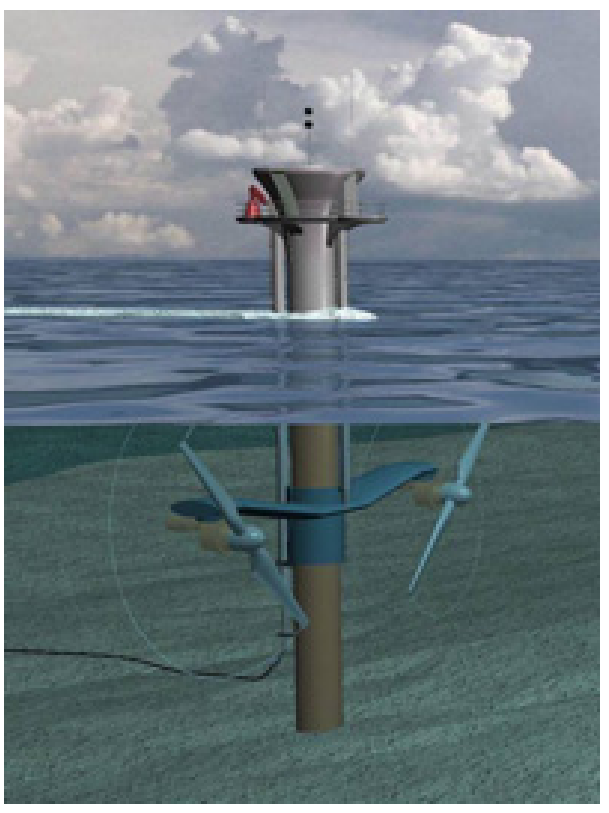

(a) Sheath type ${ }^{3}$

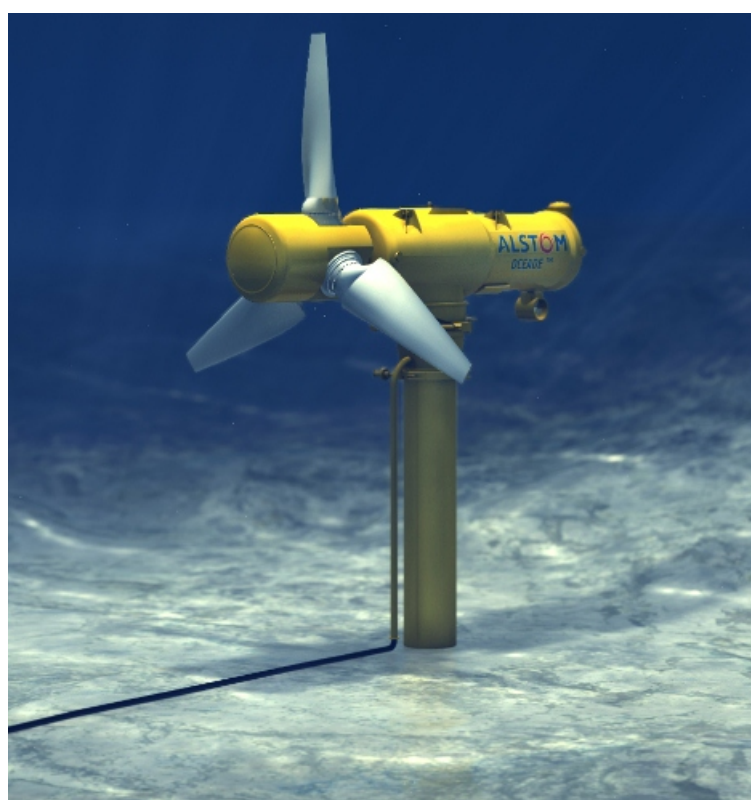

(b) Top mounted monopile ${ }^{4}$

Figure 1.3: Types of support structures.

\subsubsection{Support structure shapes}

The major reason for the drag on the support structures is due to flow separation behind the bluff bodies causing vortex shedding in their wakes. This results in pressure drag on these structures which changes with the Reynolds number of the flow. Two different support structure cross-section shapes - circular and elliptical - will be discussed in the context of their drag.

\subsubsection{Circular cylinder}

A circular cylindrical support structure is the most commonly used support structure for wind turbines and most conceptual designs of tidal turbines. The circular cylinders are only one of the most widely studied bluff body flows as discussed in Sarpkaya and Isaacson (1981) and Blevins (1990).

\footnotetext{
${ }^{3} \mathrm{http}: / / \mathrm{www}$.alternative-energy.com/tidal-energy/tidal-stream.html

${ }^{4} \mathrm{http}: / /$ www.energybc.ca/tidal.html
} 
The drag on a circular cylinder is dependant on the boundary layers and the flow separation behind the cylinder, the physics of which changes with the Reynolds number. As the Reynolds number increases, the flow separation behind the cylinder changes from laminar regime to turbulent regime which affects the drag coefficient. The laminar flow separation has insufficient momentum in the boundary layer to flow against the adverse pressure gradient. Turbulent boundary layers have higher momentum than laminar boundary layers and can sustain higher adverse pressure gradients (Zdravkovich, 1997). This causes a delay in flow separation and a change in the drag coefficient of the cylinder. The flow regime between the laminar and turbulent regime is the transition regime when there is a sudden drop in the drag coefficient of the cylinder and is known as Drag crisis; see fig 6.7a. This is caused due to the sudden transition of boundary layer from laminar to turbulent. The advantage of drag crisis is utilized in certain applications, but in the case of tidal turbines, as the channel velocity varies during the tidal cycle, the drag coefficient of the cylinder also varies. Also the roughness on the cylinder surface due to the marine environment negates the advantage of drag crisis to a large extent. The roughness of a cylinder is measured as the ratio of the thickness of the marine growth on the cylinder surface to the cylinder diameter $(r / D)$. As the roughness on the cylinder increases, the drag coefficient almost reaches its original value; see fig $6.7 \mathrm{~b}$. This makes it considerably harder to reduce the drag coefficient of the cylindrical support structure.

\subsubsection{Elliptical structure}

An ellipse has a considerably lower drag coefficient than that of a circle. An ellipse has a drag coefficient of 0.6 but similar to a circle, this changes with Reynolds number resulting in drag crisis; see fig 6.7a. Unlike a cylinder wherein the angle of attack does not really affect the flow, for an ellipse, a change in the angle of attack can significantly increase the 


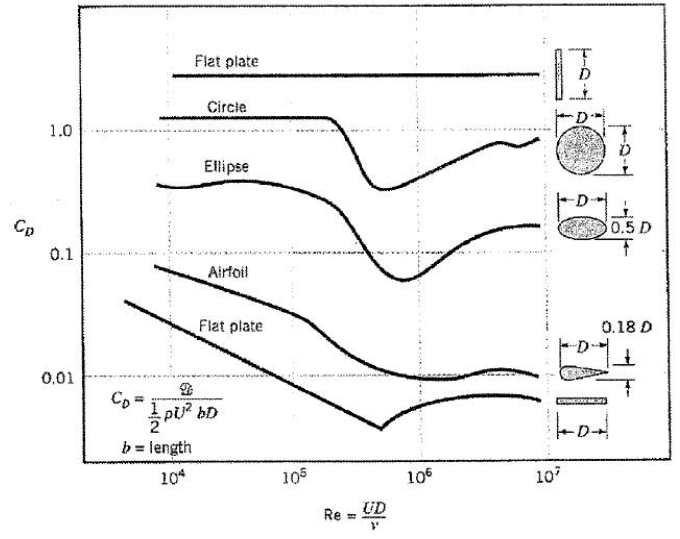

(a) Drag coefficients of various support structure shapes as a function of Reynolds number compared with a flat plate (Munson et al., 2002).

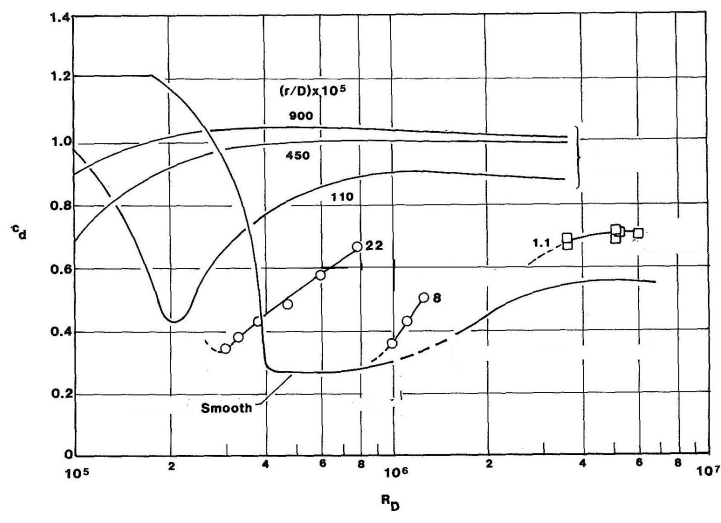

(b) Effect of surface roughness on cylinder drag coefficient as a function of Reynolds number (Polhamus, 1984)

Figure 1.4

drag on the structure. This may not be a major problem in most tidal flows where the flow is bi-directional but may be a concern when the flow direction is more irregular. Also with an ellipse, the eccentricity of the ellipse is another factor to be considered while designing an elliptical structure. Dikshit (1970) has done an experimental study on flow past an ellipse for various angles of attack and eccentricities, and has shown that these factors can cause a significant change in the drag coefficient. In terms of drag, the support structure needs to be as slender as possible but from a structural point of view, it should be able to support the turbine and withstand the forces from the currents, and hence an optimum thickness must be determined.

Even though the support structures are aligned to the flow for a zero angle of attack, since they are in the wake of the turbine blades, there is a swirling flow onto them which can change the angle of attack on the support structures (ellipse) causing an increase in drag and lift. But this swirling flow is expected to change the angle of attack by only about $5^{\circ}$ which may not be as significant as ellipse has a considerably lower drag coefficient compared to that of a circle. This will be examined in detail in this thesis. 


\subsubsection{Effects of support structure on turbine system}

\subsubsection{Thrust on the turbine system}

Walker (2014) discussed the variation in turbine performance in the presence of a support structure and the influence of support structure diameter on power output. He considers different configurations of the support structure but all of them have the same cylindrical cross-section.

Mason-Jones et al. (2013) have shown the influence of support structure on the torque, power output and axial thrust on a turbine system using CFD models. They discuss about the significant losses in power output due to the presence of support structures and even though different support structure shapes were considered in a uniform flow, only a circular

cylindrical support structure was considered in sheared flow. Also the support structure was considered to be present both above and below the nacelle (i.e. the turbine is fixed at the vertical centre of the support structure).

In this thesis, different support structure shapes were considered and their effect on the axial thrust, torque and power output were studied using CFD.

\subsubsection{Tower shadow}

The presence of support structure disrupts the flow around it whose influence is also felt by the turbine blades. This is known as tower shadow and it has a major influence on turbines (Reiso et al., 2011). Most of the work done on tower shadow so far has been for wind turbines. In the present thesis, tower shadow is analyzed in the case of tidal turbines.

Fruh et al. (2008) have shown that in the immediate vicinity upwind of the support structure, the presence of support structure changes the effective angle of attack of the airfoil cross-section of the turbine blades and also causes a decrease in the flow velocity. This results in a sudden dip in the torque when a turbine blade is in that region which 
in-turn results in a dip in the power output (Dolan and Lehn, 2006). For a three bladed turbine, these fluctuations (dips) occur at three times the rotor frequency which is commonly known as $3 p$ frequency.

Sescu and Andersen (2011) have shown the influence of tower shadow using CFD for three cases, free rotor without support structure, an upwind rotor and a downwind rotor for a cylindrical support structure. The overall fluctuations are analyzed and as expected these fluctuations are highest for a downwind turbine but still quite significant for an upwind turbine compared to a free rotor. Streamlining the support structure might result in a lower reduction in flow speed around the structure and also the effective angle of attack would not vary as much as that for a cylindrical support structure. This would reduce the range of decrease in torque and the overall loss in power output.

The shear profile of the flow is also known to cause torque fluctuations but Dolan and Lehn (2006) have shown that the tower shadow effect has greater influence on these fluctuations than shear profile in the case of wind turbines.

\subsubsection{Flow velocity recovery}

The flow past a turbine rotor and its support structure results in a decrease in flow velocity in their wake. This flow velocity deficit must recover before the flow reaches the next row of turbines in a turbine array and hence this is an important factor in spacing between turbines. Ideally the spacing between turbines should be minimized to accommodate the maximum number of turbines and hence streamlined support structures might be an option.

Myers and Bahaj (2009) have studied experimentally, the flow past a turbine rotor and its support structure, both when the rotor is operational to get the combined wake and when the rotor is constrained to rotate to obtain just the support structure wake. The visualization 
of wake indicates the significant role played by support structure in the velocity deficit behind the turbine system.

Malki et al. (2011) have studied the influence of inflow velocity on velocity deficit behind a turbine and its support structure in the downstream and lateral directions using a BEM-CFD model. In the current work, the velocity deficit in the case of various turbine systems with different support structures was analyzed which would be useful in determining the optimum array spacing in turbine farms.

\subsection{Aim and Objectives}

This thesis is confined to horizontal axis tidal turbines which are mounted on different monopile support structures. The main aim of this thesis is to study the influence of support structure drag on the overall power reduction from a tidal farm. The impact of installing too many turbines, and particularly those with high support structure drag, that may have a potentially unacceptable adverse effect on the flow rate will be discussed. Further, the effects of introducing an environmental constraint on flow rate reduction will be analysed.

The first objective of this thesis is to develop an analytical model to obtain the power output from a tidal farm, wherein the turbine rotors are mounted on difference support structure cross-sectional shapes. This model includes the power-capping mechanism used by turbines but does not take into consideration the viscous interaction effects between a rotor and its support structure.

The second object is to set-up a computational model to study the turbine performance in the power-capping zone by pitching the blades to feather. Also since the tidal channel flow has a shear in its velocity profile, the influence of shear on turbine performance is studied by comparing it to the same turbine in a uniform flow. 
The third objective is to study the flow interaction effects between the rotor and its support structure using Computational Fluid Dynamics (CFD) for different support structure shapes for a range of tidal velocities including the power capping zone.

The final objective of this thesis is to reassess the farm power output using the initial analytical model based on the new data obtained from CFD.

\subsection{Thesis Outline}

The main objective of this work is to indicate the importance of support structures in the reduction of the power output from a tidal farm. An analytical model is used to obtain an estimate of the power output from a tidal channel. In this model, the power and thrust coefficients of the rotor, and the support structure drag coefficient are approximated without considering any interaction effects between the rotor and the support structure. Hence CFD is used to study the flow field and the interaction effects between the rotor and support structure, and the resulting force coefficients from CFD are used to re-estimate the power output from the tidal farm to study the change in power output, with and without support structures. The rest of the thesis is organised into seven chapters.

The analytical model is introduced in Chapter 2 which gives an initial estimate of the power output from a tidal farm. The impact of support structures is presented by analyzing the reduction in the farm power output in the presence of support structures. Also the high opposing thrust due to turbine rotors and their support structures can decrease the channel flow velocity considerably which might have an adverse affect on the environment which is also discussed in this chapter. Since the analytical model cannot predict the interaction effects between the rotor and support structure, CFD needed to be used to obtain a better estimate of the rotor power and thrust coefficients, and the support structure drag coefficient. 
The numerical methods used in CFD are discussed in Chapter 3, particularly the solvers used in OpenFOAM (2015), which was used to simulate the rotor-support structure interactions.

The experimental data on interaction effects between tidal turbines and their support structures are limited and hence a building block approach was used in Chapter 4 to validate the results. In a Building block approach, the complexity of a system is reduced by decomposing it into smaller components and validating these components. In the present study, the entire turbine system is decomposed into support structure and rotor, and each of them are validated separately.

In Chapter 5, a full-scale turbine rotor in a tidal channel is simulated using CFD, and the flow and loading characteristics in the power capping zone due to blade pitching are discussed without including a support structure. A real tidal channel has a shear profile in velocity instead of a uniform profile. Hence before analyzing blade pitching, the effect of shear velocity profile on the turbine is also studied.

In Chapter 6, two different monopile support structures, with cylindrical and elliptical cross-sections, are considered and their influence on the flow is studied. The influence of the support structure on the rotor is studied by analysing the forces and the angle of attack distribution along the rotor blades, and then the influence of the rotor on the support structure is discussed by investigating the forces on the support structure. The CFD simulations provide a better estimate of the rotor power and thrust coefficients along with the support structure drag coefficient which were approximated previously in Chapter 2 without considering the interaction effects.

In Chapter 7, the new force coefficients obtained from CFD were used in the reassessment of the farm power output using the analytical model of Chapter 2 in order to obtain a better farm power estimate in the presence of different support structures. 
The conclusions of this work are presented in Chapter 8 along with possible future work. 


\section{Chapter 2}

\section{Farm power output analysis}

The farm power output of a tidal channel in the presence of various number of turbines and different support structures is analyzed in this chapter. The influence of three major factors on the opposing thrust presented to the channel is considered: 1) thrust from the turbine, 2) drag from the support structure and 3) seabed friction. In the next section, the influence of turbine rotor and support structure thrust on Power-Thrust curves and turbine efficiency curves is analyzed. Later, the influence of these factors on the number of turbines that can be installed in a channel and the total power output from the farm are studied for different channel characteristics. Also, it is shown that installing too many turbines can have a potentially unacceptable adverse effect on the channel flow rate and the consequence of introducing a flow rate reduction constraint is explored.

\subsection{Turbine Performance}

Consider a typical tidal turbine rotor of $20 \mathrm{~m}$ diameter. At very low channel velocities below the cut-in speed, here $0.5 \mathrm{~ms}^{-1}$, there is not enough torque on the rotor and there is no power output. Above the cut-in speed, the turbine power varies cubically with the 


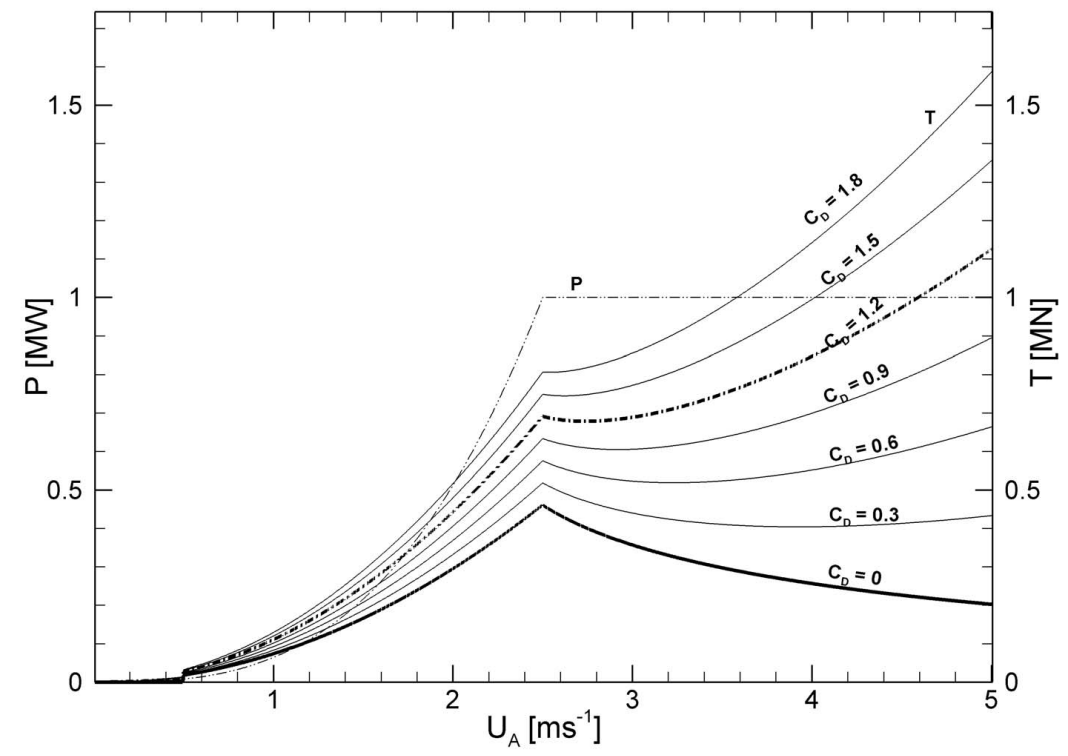

Figure 2.1: Power, P, curve and Thrust, $\mathrm{T}$, curves for a turbine with different support structure drag coefficients, $0 \leq C_{D} \leq 1.8$, as a function of channel velocity, $U_{A}$; area ratio $\chi=0.2$.

channel velocity and turbines with a power coefficient, $C_{P}=0.4$ are considered. As the channel velocity increases, the turbine power output increases until it reaches its rated power, here $1 \mathrm{MW}$, at a rated flow speed of $2.5 \mathrm{~ms}^{-1}$, and at higher flow speeds the turbine power is limited to this rated power in order to protect it from damage at high flow speed loadings, see figure 2.1 .

The turbine exerts an opposing thrust, $F_{T}$, on the flow which may be found from

$$
C_{T T}=\frac{F_{T}}{\frac{1}{2} \rho A_{t} U_{A}^{2}}
$$

where $C_{T T}$ is the total turbine thrust coefficient, $\rho$ is the density of water $\left(1025 \mathrm{kgm}^{-3}\right)$, $A_{t}$ is the rotor swept area and $U_{A}$ is the channel flow velocity. To evaluate the turbine thrust, $C_{T T}$ must first be determined for which Linear Momentum Actuator Disk Theory (LMADT) is used to relate the power coefficient, $C_{P}$, and total thrust coefficient, $C_{T T}$, of 
a turbine to its axial induction factor, $a_{i}$ :

$$
\begin{aligned}
& C_{T T}=4 a_{i}\left(1-a_{i}\right) \\
& C_{P}=4 a_{i}\left(1-a_{i}\right)^{2} ;
\end{aligned}
$$

Given the power curve of the turbine, figure 2.1, the power coefficient can be evaluated for the entire range of velocities using

$$
C_{P}=\frac{P}{\frac{1}{2} \rho A_{t} U_{A}^{3}}
$$

and thus $C_{T T}$, and hence the thrust of the turbine, can be derived from $C_{P}$ through equations (2.1) to (2.3).

The turbines are assumed to be fixed to the seabed by means of a support structure. Hence, along with the turbine thrust, the drag force from the support structure of the turbine must be included to study the overall effect of the turbine system on the flow. The thrust from the support structure is given by:

$$
F_{S}=C_{D} \frac{1}{2} \rho A_{s} U_{A}^{2}
$$

where $C_{D}$ is the drag coefficient of the support structure and $A_{s}$ is the frontal area of the support structure which can be related to the rotor frontal area $A_{t}$ through an area ratio, $\chi$,

$$
\chi=\frac{A_{s}}{A_{t}}
$$

A representative rotor of diameter, $d=20 \mathrm{~m}$, supported on a cylindrical tower of diameter $3 \mathrm{~m}$ and height $20 \mathrm{~m}$ is considered, leading to $\chi=0.2$. The most commonly used shape 
for support structures will likely be a cylinder for which $C_{D}=1.2$ across a wide range of Reynolds numbers. However, to study other support structures, $C_{D}$ is varied over a range of values to see its effect on the overall thrust of the turbine system, $T$; see figure 2.1. It should be noted that in this study, $\chi$ is maintained constant even when the drag coefficient of the support structure is varied. Further, it is the product $\chi C_{D}$ that is important, and hence varying $C_{D}$ may be considered as equivalently varying the structure frontal area, $\chi$.

The efficiency of a turbine is another important factor to be taken into consideration when designing a turbine. All of the energy removed from the flow cannot be converted into useful power as some power is lost in overcoming the drag of the support structure and there are necessary mixing losses required to mix the turbine core and bypass flows further downstream. The efficiency of a turbine is given by

$$
\eta=\frac{U \text { seful Power }}{\text { Power removed from the flow }}=\frac{P}{T U_{A}}
$$

where the algebraic expression for $\eta$ assumes that the free surface does not deform. The efficiency curves are derived from the power and thrust curves of the turbine; see figure 2.2. The theoretical curve is obtained from LMADT. For $U_{A} \leq 2.5 \mathrm{~ms}^{-1}$, power is below rated power and $C_{P}=0.4$ is held constant and thus efficiency remains constant over this velocity range. From figure 2.1, it can be observed that the overall thrust for the $C_{D}=0$ case (i.e in the absence of support structure) decreases for $U_{A}>2.5 \mathrm{~ms}^{-1}$, whilst output power remains constant. This indicates that the efficiency of the turbine is always increasing for velocity higher than $2.5 \mathrm{~ms}^{-1}$ as seen in figure 2.2. For all the other curves, $C_{D}>0$, the overall thrust decreases until a certain velocity is reached after which the increasing support structure drag leads to a rise in the overall thrust as shown in figure 2.1. This effect can also be observed in the efficiency curves where the efficiency initially increases through power capping before decreasing again at higher flow speeds for which 


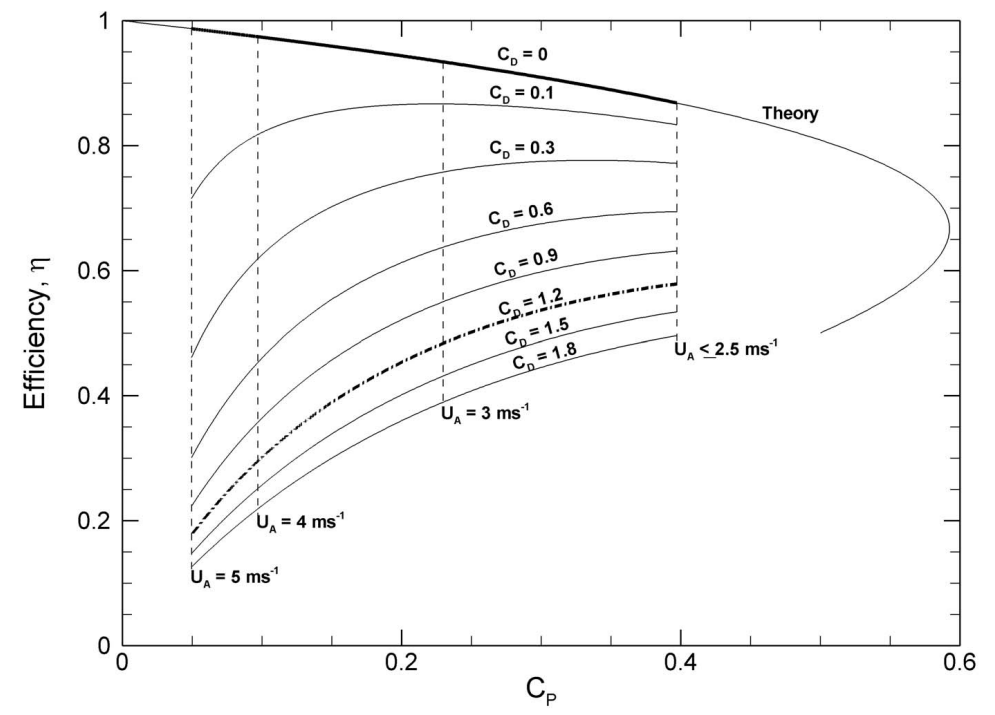

Figure 2.2: Hydrodynamic efficiency, $\eta$, curves for a turbine with different support structure drag coefficients, $0 \leq C_{D} \leq 1.8$, and area ratio $\chi=0.2$. The theoretical curve from LMADT is also plotted for comparison. Dashed lines indicate channel velocities.

the support drag becomes more important; see figure 2.2.

From the efficiency curves, it can be observed that a small change in the drag coefficient of the support structure has a large influence on the efficiency of energy extraction. For $C_{D}=0$ efficiency remains above $85 \%$ for all flow speeds, whilst for $C_{D}=1.2$, at $U_{A}=2.5 \mathrm{~ms}^{-1}$, the efficiency is around $60 \%$ and at $U_{A}=5 \mathrm{~ms}^{-1}$, the efficiency falls as low as $20 \%$. This is a significant drop in efficiency and it underlines why reducing the support structure drag is important in order to improve the overall efficiency of the turbine, noting, as will be shown in this chapter, that it is the overall thrust imposed on a channel flow, and therefore the power that can be removed (not generated) from the flow is limited.

\subsection{Channel Dynamics}

A simplified channel of rectangular cross section area, $A_{c}$, of width, $W=1 \mathrm{~km}$, depth, $h=40 \mathrm{~m}$ and length, $L=5 \mathrm{~km}$ is considered. The 1D Euler equation for flow in the 
channel is given by

$$
\frac{\partial u}{\partial t}+u \frac{\partial u}{\partial x}+g \frac{\partial \zeta}{\partial x}=-F
$$

where $u$ is the flow velocity, $\zeta$ and $F$ are the driving head and resistive force per unit mass respectively. The term $F$ represents all the resistive forces which oppose the flow, and includes the thrust of the turbines, drag from turbine support structures and sea bed friction.

Following Garrett and Cummins (2005), the flow velocity is re-written in terms of the volume flow rate $\left(Q=A_{c} u\right)$, and integrating equation (2.8) along the channel yields

$$
c \frac{d Q}{d t}-g \zeta_{0}=-\int_{0}^{L} F d x-\frac{1}{2} u_{e}\left|u_{e}\right|
$$

where $c$ is the coefficient given by $\int_{0}^{L} A_{c}^{-1} d x, \zeta_{0}=\zeta(L)-\zeta(0)$ is the head difference along the channel and $u_{e}$ is the channel exit flow velocity. As in Garrett and Cummins (2005) the flow is assumed to be drawn into the channel through a smooth entry.

The opposing force due to sea bed friction is given by

$$
F_{b}=C_{f} \frac{1}{2} \rho A_{b} U_{A}\left|U_{A}\right|
$$

where $C_{f}$ is the bed-friction coefficient and $A_{b}$ is the sea bed area.

The total opposing force, $\bar{F}=\rho L A_{c} F$, in the channel is given by

$$
\bar{F}=n\left[C_{T} \frac{1}{2} \rho A_{t} U_{A}\left|U_{A}\right|+C_{D} \frac{1}{2} \rho A_{s} U_{A}\left|U_{A}\right|\right]+C_{f} \frac{1}{2} \rho A_{b} U_{A}\left|U_{A}\right|
$$

where $n$ represents the number of turbines installed in the channel. The first two terms on the right hand side are due to the presence of turbines and their support structures respectively and the third is due to seabed friction. 
In equation (2.9), it was assumed that the opposing forces $(F)$ from the channel were acting uniformly throughout the channel and hence independent of the position along the length of the channel. Also as the flow exits the channel smoothly, the kinetic energy at exit would be small and hence the last term in the equation was neglected. Representing the driving head as a sinusoidal tide with amplitude $a$ and frequency $\omega$,

$$
c \frac{d Q}{d t}-g a \cos (\omega t)=-F L
$$

The term on right hand side of equation (2.12) is represented by $\widehat{F}=F L=\frac{\bar{F}}{\rho A_{c}}$, and from equation (2.11),

$$
\widehat{F}=\frac{1}{2} U_{A}\left|U_{A}\right|\left[\frac{n}{A_{c}}\left[C_{T} A_{t}+C_{D} A_{s}\right]+C_{f} \frac{L}{h}\right]
$$

Introducing the total area ratio, $\xi=n A_{t} / A_{c}$, which represents the ratio of total rotor frontal area to channel cross-sectional area (note that this is not the same as the global blockage ratio as the rotors are not constrained to lie in the same plane) and substituting for area ratio, $\chi$, equation (2.12) is rewritten non-dimensionalizing the flow equation with $t^{\prime}=\omega t, Q^{\prime}=Q / Q_{0}$ (where $Q_{0}=g a / \omega c$ is the magnitude of the volume flux in the absence of flow resistance) yields

$$
\frac{d Q^{\prime}}{d t^{\prime}}-\cos t^{\prime}=-\frac{1}{2} Q^{\prime}\left|Q^{\prime}\right| \frac{1}{F r_{w}^{2}}\left(\xi\left(C_{T}+\chi C_{D}\right)+C_{f} \frac{L}{h}\right)
$$

where $F r_{w}=\omega L / \sqrt{g a}$ is a non-dimensional grouping taking a similar form to the Froude number. The solution to the above equation is obtained through Runge-kutta time marching. The time step is chosen such that one tidal cycle time period is divided into thousand times. The model was run until the difference between two consecutive cycles 
was less than the set tolerance of $10^{-7}$. The values over the last cycle were averaged for the power and thrust analysis. For all cases investigated in this work, a representative seabed friction coefficient of $C_{f}=0.002$ (Massey, 1998) is considered. A 50\% change in $C_{f}$ would change the peak power by about $10 \%$.

Whilst $C_{T}, C_{D}, \xi$ and $\chi$ describe the tidal energy farm, $F r_{w}, C_{f}$ and $L / h$ characterize the underlying channel flow. In this chapter, tidal energy extraction will be explored as a function of the channel parameters, $F r_{w}$ and $L / h$. Both $F r_{w}$ and $L / h$ have a dependency on $\mathrm{L}$, and hence $F r_{w}$ is varied by changing tidal amplitude, $a$, and maintaining $\mathrm{L}$ constant, so that $L / h$ remains constant. $L / h$ is varied by changing $\mathrm{L}$, which would also change $F r_{w}$ and hence tidal amplitude, $a$, is also varied in this case such that $F r_{w}$ remains constant. $F r_{w}$ and $L / h$ may be viewed as indicators of the volume flow rate (or velocity) through the channel. A lower $F r_{w}$ corresponds to a higher tidal amplitude, $a$, or a shorter channel, $L$, both of which imply a larger relative head to volume of water within the channel which results in higher flow velocity. Similarly a higher $L / h$ implies a relatively shallow channel and hence the same head trying to drive a smaller volume of water resulting in a higher flow velocity.

Based on the dimensions of the channel described earlier, $L / h=125$ and $F r_{w}=$ 0.478 corresponding to a tidal amplitude, $a=0.25 \mathrm{~m}$. For these values, the equations are evolved in time for $Q^{\prime}$ to obtain the volume flow rate, $Q$, and thus the farm power and thrust may be determined. Figure 2.3 shows the volume flow rate, power and thrust curves depicted for one tidal cycle for the case, $\chi=0.1$ and $n=34$ (or $\xi=0.27$ ). The physical quantities are plotted here for clarity of interpretation and non-dimensional representation will be addressed later in the chapter. The two thrust curves represent the thrust of the turbine alone $\left(F_{T}\right)$, and the combined thrust of turbine and support structure $\left(F_{T}+F_{S}\right)$. The volume flow rate is almost sinusoidal whereas the power curve exhibits 


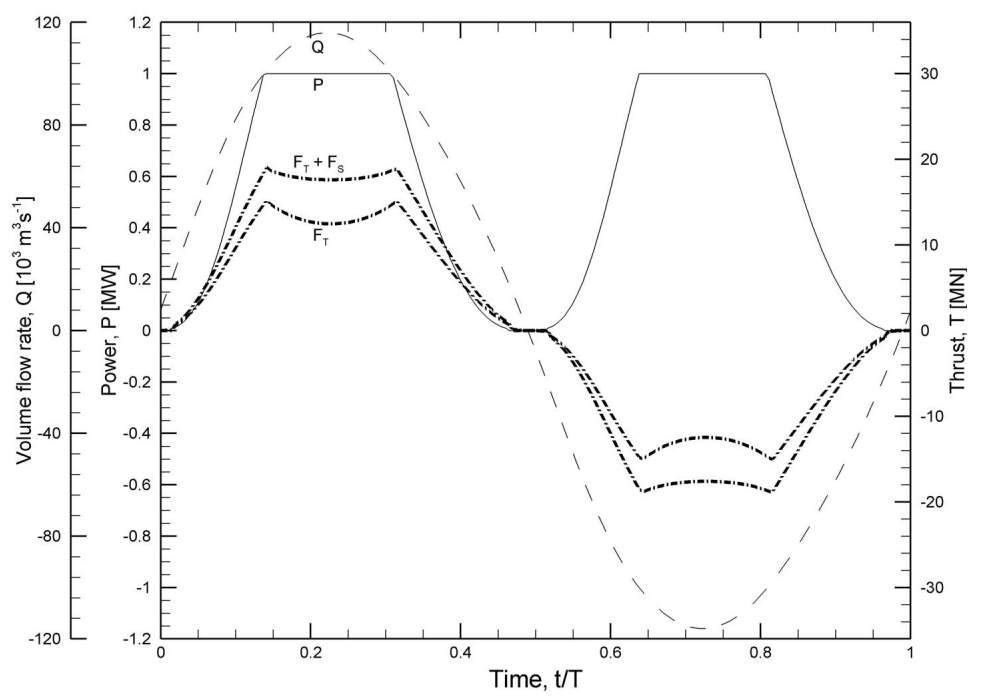

Figure 2.3: Volume flow rate ,Q, power, $\mathrm{P}$, and thrust, T, curves plotted over one tidal cycle $\left(\chi=0.2, n=34, C_{D}=1.2, F r_{w}=0.478, L / h=125, C_{f}=0.002\right)$.

a capped behaviour due to the rated power constraint at high flow speeds. Similarly the thrust curves also exhibit non-monotonic behaviour above rated flow speeds. The thrust of the turbine decreases in this power capping zone due to blade feathering whereas the thrust from the support structure increases due to increase in flow velocity. Hence, the sum of turbine and support structure thrust may increase or decrease depending on the relative influence of the turbine thrust and the support structure drag. It remains almost constant for a support structure drag coefficient of 1.2 as shown in figure 2.3. From these curves, the time averaged farm power and thrust can be determined.

For the channel considered, $F r_{w}=0.478$ and $L / h=125$, the time averaged total farm power output over one tidal cycle, $\left\langle P_{f}\right\rangle$, is then computed for a range of number of turbines and support structure drag coefficients; see figure 2.4. These power curves exhibit a non-linear behaviour which is expected due to the non-linearity of the opposing force term in the governing equation. The opposing thrust force in the channel reduces the channel flow rate from its initial level. As the number of turbines installed in the channel increases, the opposing thrust force increases and the maximum channel velocity $\left(U_{A \max }\right)$ 
observed through the cycle decreases; see figure 2.4 . The total farm power output initially increases with increasing number of turbines but the decreasing channel flow velocity leads to a relatively smaller increase in power delivered per turbine added, and eventually, even larger reduction in flow rate results in reduced farm power. Hence, a peak in the farm power output from the channel was observed at a certain number of turbines.

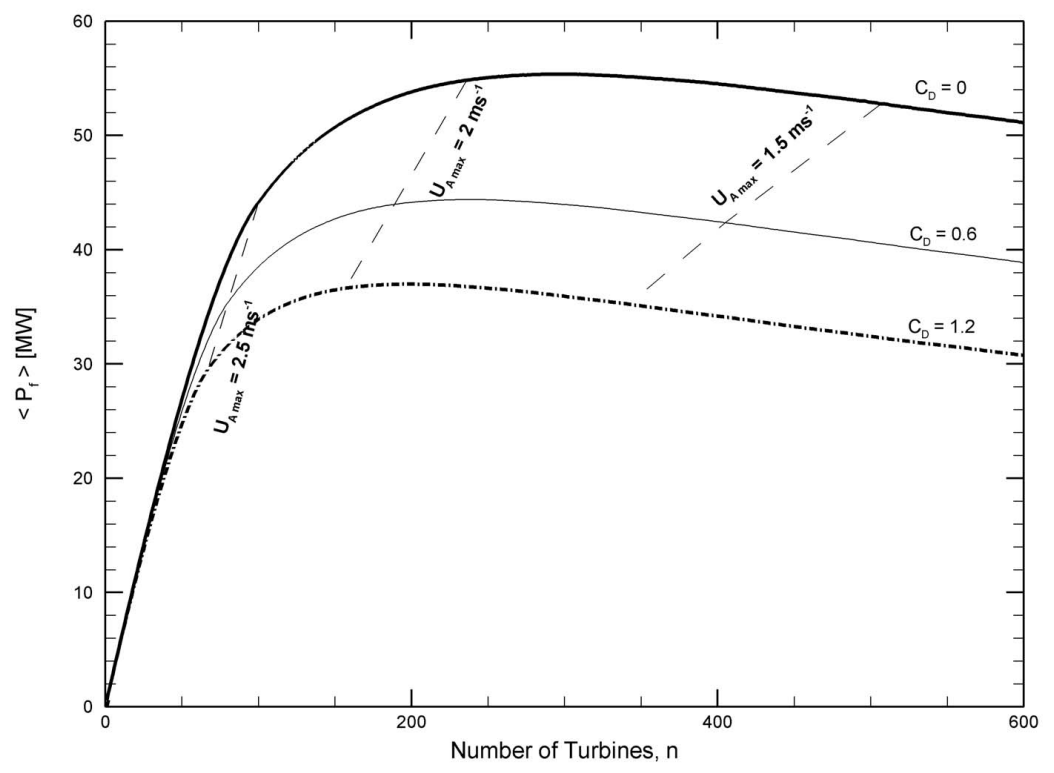

Figure 2.4: Time-averaged total farm power output, $\left\langle P_{f}\right\rangle$, as a function of the number of turbines installed and support structure drag coefficient. The dashed lines indicate the maximum channel velocity $\left(U_{A \max }\right)$ attained through the tidal cycle $\left(\chi=0.2, F r_{w}=\right.$ $\left.0.478, L / h=125, C_{f}=0.002\right)$

From figure 2.4, it can also be observed that there is a drastic reduction in the total farm power output when the support structure drag is included and this drop is higher with larger drag coefficients. The peak attainable power output drops by almost $40 \%$ in the presence of a typical cylindrical support structure $\left(C_{D}=1.2\right.$ and $\left.\chi=0.2\right)$ compared to that without any support structure. Also as the drag coefficient of the support structure increases, the peak power output is obtained using fewer turbines. This infers that the optimum number of turbines that can be installed in a channel is strongly a function of 
the support structure drag, and that the capacity of a channel, in terms of the maximum number of turbines that can be installed before reducing farm output, is dependent on careful design of support structures.

\subsection{Power vs thrust}

The analysis of the previous section is discussed in terms of the time averaged absolute value of the opposing thrust, $\left\langle\left|T_{c}\right|>\right.$, from the channel as opposed to the number of turbines installed. Two non-dimensional parameters are introduced to describe the total power output and the opposing thrust in the channel

$$
\begin{gathered}
C_{P c}=\frac{<P_{f}>}{\rho g a Q_{0}} \\
C_{T c}=\frac{<\left|T_{c}\right|>}{\rho g a A_{c}}
\end{gathered}
$$

$C_{P c}$ and $C_{T c}$ are the non-dimensional channel power and thrust coefficients. The term $\rho g a Q_{0}$ is an indicator of the power available in the channel. Garrett and Cummins (2005) showed that in the case of linear flow resistance the maximum power that can be generated from a tidal channel is $\frac{1}{4} \rho g a Q_{0}$ so that $C_{P c}$ becomes a measure of the power that can be generated from the channel and takes a maximum value of $1 / 4$.

The variation of $C_{P c}$ with $C_{T c}$ for various tidal amplitudes, $a=0.125 \mathrm{~m}, 0.25 \mathrm{~m}, 0.5$ $\mathrm{m}$ is shown in figure 2.5. If cut-in and rated flow speed constraints are neglected, i.e. $P \propto U_{A}^{3}$, then there is a unique relation between $C_{P c}$ and $C_{T c}$ for all $a$; the curve marked 'unconstrained'. Once the non-linearities of cut-in and rated power constraints are introduced, the farm performance is observed to be a function of tidal amplitude because the 


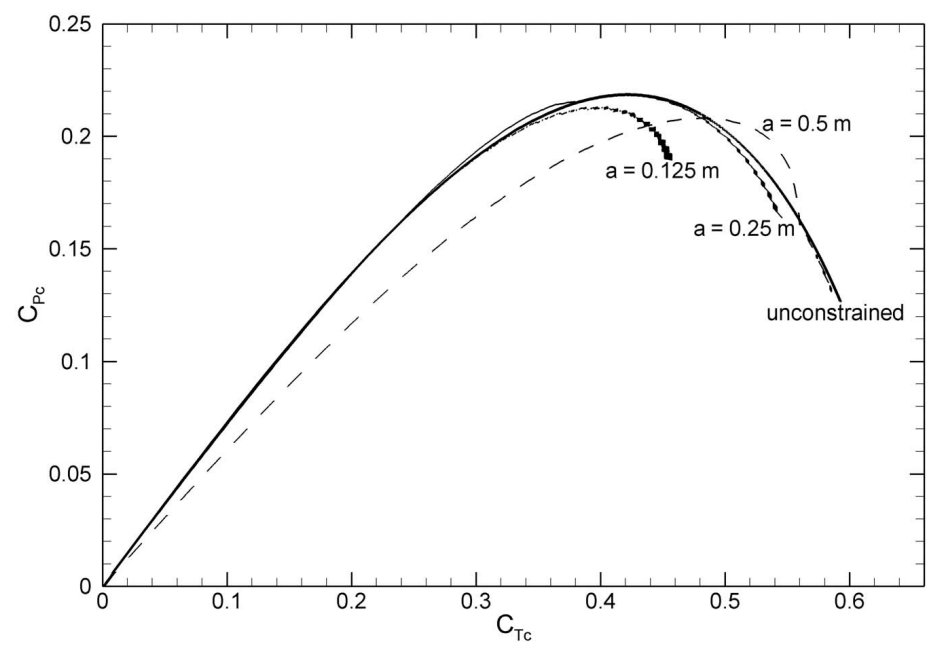

Figure 2.5: Farm performance, power-to-thrust, for various tidal amplitudes, and an unconstrained case for which turbine cut-in and rated speeds are neglected such that $P \propto U_{A}^{3}$ $\left(L / h=125, \chi=0.2, C_{D}=1.2, C_{f}=0.002\right)$.

range of flow speeds seen by each turbine and hence the proportion of the tidal cycle spent above cut-in and rated flow speeds is different.

The influence of the two non-dimensional parameters, $F r_{w}$ and $L / h$, on farm performance, $C_{P c}$ and $C_{T c}$, is shown in figures 2.6 and 2.7 respectively. The influence of tidal amplitude on channel power coefficient $C_{P c}$ through varying $F r_{w}$ is shown in figure 2.6. Initially when there are no turbines installed, $C_{P c}=0$, the opposing thrust from the channel is due to seabed friction alone, and hence $C_{T c}$ is non-zero even when $C_{P c}$ is zero. This thrust can be determined from the value of $C_{T c}$ at $C_{P c}=0$; see figure 2.6. $C_{T c}$ due to seabed friction increases as $F r_{w}$ decreases (due to an increase in the corresponding channel flow velocity).

As identified previously the total power output, $C_{P c}$ is seen to decrease with an increase in support structure drag coefficient, inferring that the parasitic power loss is higher in the presence of support structures.

As the number of turbines installed increases, the maximum channel velocity is re- 


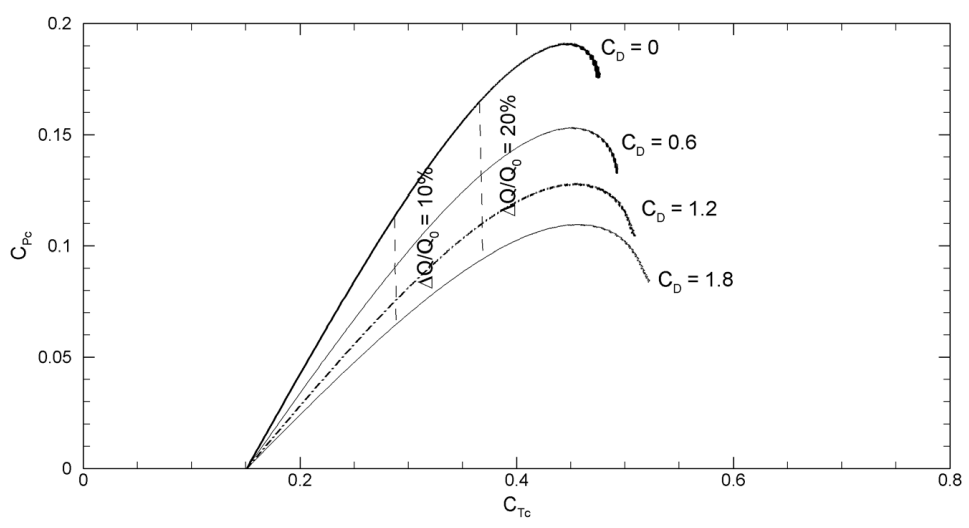

(a)

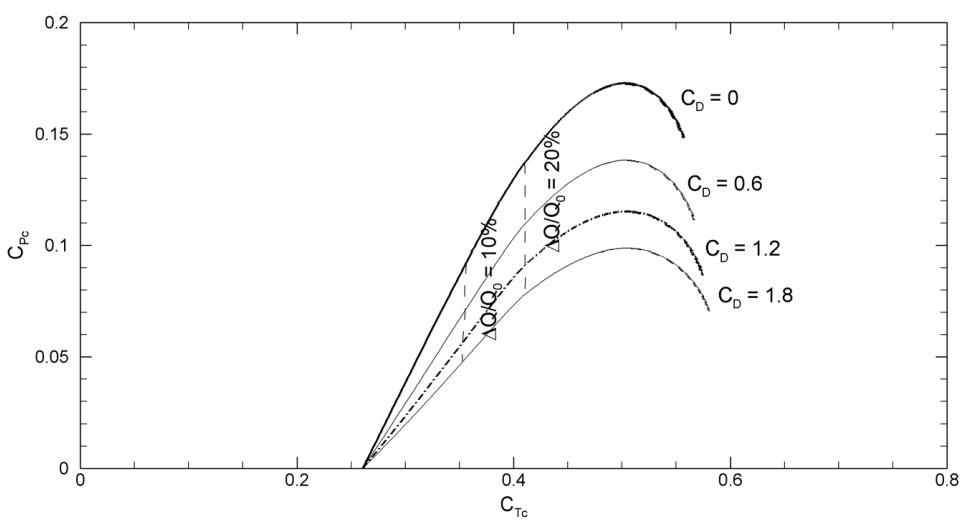

(b)

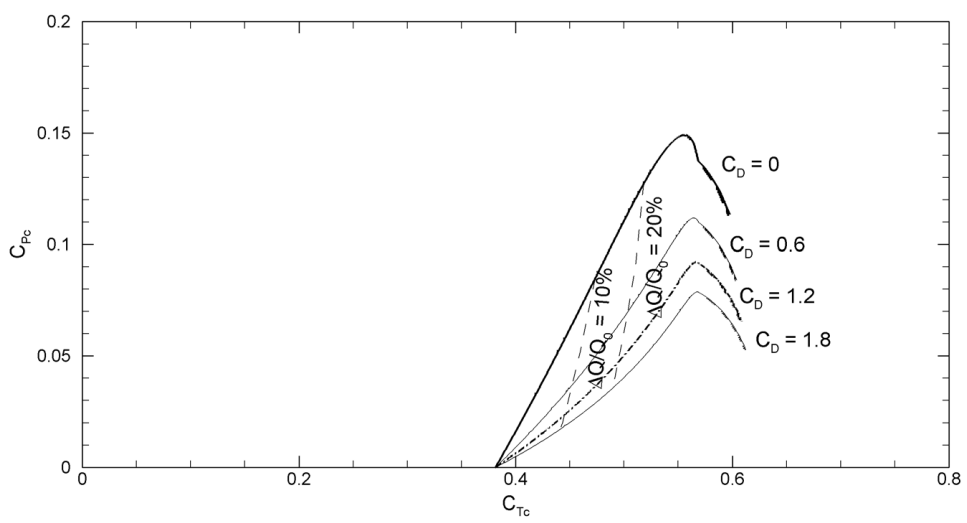

(c)

Figure 2.6: Channel power coefficient, $C_{P c}$, as a function of channel thrust coefficient, $C_{T c}$, for (a) $F r_{w}=0.673(a=0.125 m)$, (b) $F r_{w}=0.478(a=0.25 m)$, (c) $F r_{w}=0.338$ $(a=0.5 \mathrm{~m})$. The dashed lines indicate constraints on change in the maximum volume flow rate in the channel. $\left(L / h=125, C_{f}=0.002, \chi=0.2\right)$. 
duced and this reduction in channel velocities can have a negative environmental impact. Hence the imposition of flow rate reduction constraints to not reduce the peak flow rate by more than ten and twenty percent is investigated by studying its effect on $C_{P c}$. In figure 2.6b, the two dashed lines with $\Delta Q / Q_{0}=10 \%$ and $20 \%$ indicate the permissible levels of reduction in peak volume flow rate. Even for these large permissible flow rate reductions, maximum channel power is significantly reduced below the peak achievable power.

In figure 2.6a, the lines of volume flow rate constraint (10\% and 20\%) are almost vertical and the slope of these lines changes with $F r_{w}$ as shown in figures 2.6b and 2.6c. Vertical constraint lines indicate that reducing parasitic support structure drag leads to an increase in farm time-averaged power with no increase in the overall time-averaged thrust. This situation occurs for higher $F r_{w}$ (lower flow velocity) because rated flow speeds are not reached and hence the ratio $C_{T} / C_{D}$ remains constant for each turbine. However at lower $F r_{w}$ (higher flow speeds), $C_{T} / C_{D}$ is not constant as rated flow speeds are exceeded and hence the lines of constant flow rate reduction are non-linear in $C_{P}-C_{T}$ space. The non-linearity is such that increasing structural drag more rapidly reduces generatable power at lower $F r_{w}$ (higher flow speeds) than at higher $F r_{w}$ (lower flow speeds). Indeed for the case $F r_{w}=0.338(a=0.5 m)$ a flow rate reduction constraint of $\Delta Q / Q_{0}=10 \%$ reduces maximum deliverable power by $75 \%$ as the drag coefficient increases from 0 to 1.2 .

The other non-dimensional parameter is $L / h$ and based on the dimensions of the base case channel, $L / h=125$. As can be seen from the governing equation, a higher $L / h$ indicates a higher energy flow and an increase in the flow velocity. Hence, $C_{T c}$ increases with increasing $L / h$ in the absence of turbines $\left(C_{P c}=0\right)$, i.e. due to bed friction alone; see figure 2.7. It is observed that as $L / h$ is increased and greater energy is given up to overcome bed friction, less energy is available for turbine power generation or to overcome 


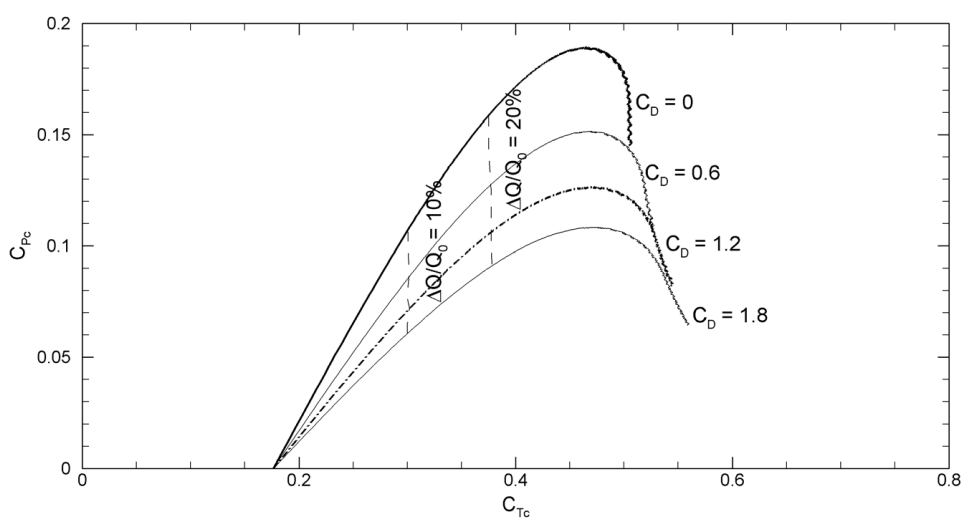

(a)

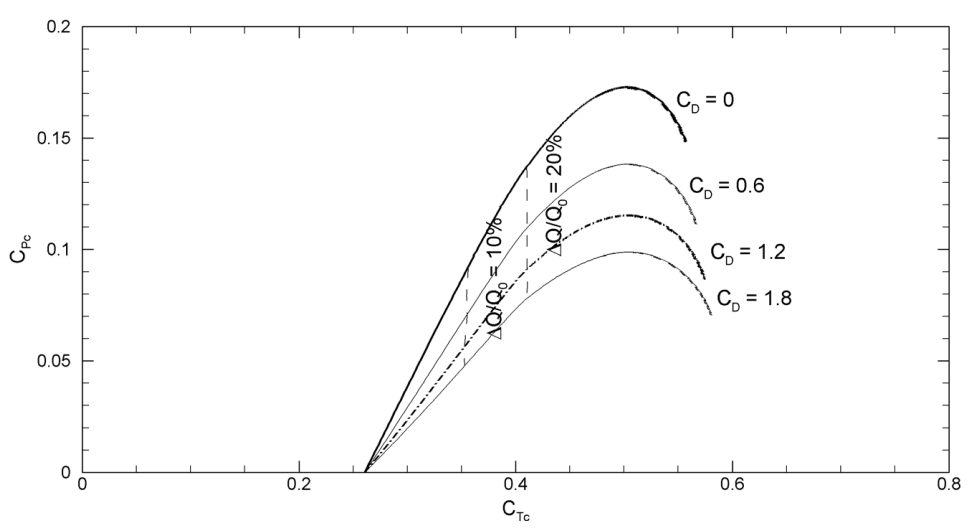

(b)

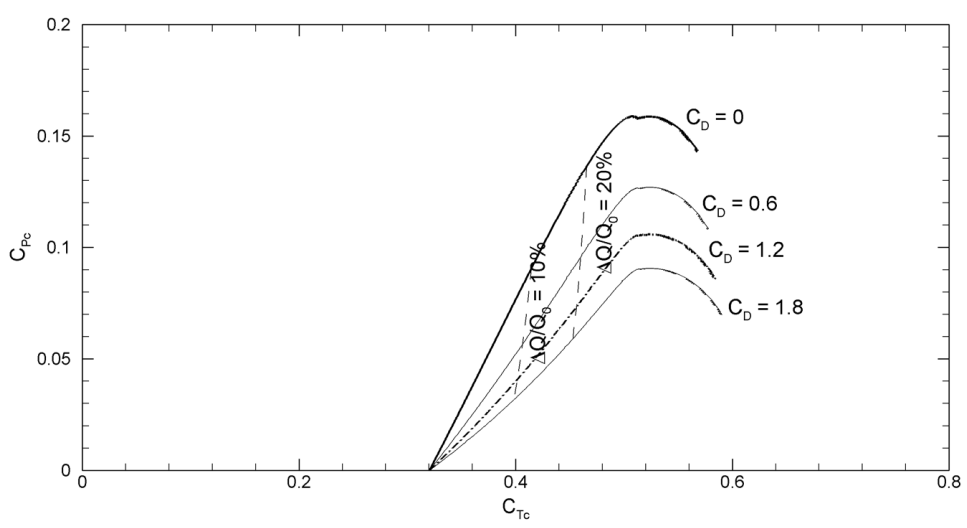

(c)

Figure 2.7: Channel power coefficient, $C_{P c}$, as a function of channel thrust coefficient, $C_{T c}$, for (a) $L / h=75$, (b) $L / h=125$, (c) $L / h=175$. The dashed lines indicate constraints on change in the maximum volume flow rate in the channel. $\left(F r_{w}=0.478, C_{f}=\right.$ $0.002, \chi=0.2)$. 
Table 2.1: Farm power output dependency on $F r_{w}$

\begin{tabular}{|l|l|l|l|l|l|l|l|l|l|}
\hline$C_{D}$ & $F r_{w}$ & $U_{A}$ & \multicolumn{2}{|c|}{$P_{f \max }$} & \multicolumn{2}{l|}{$P_{f \Delta Q / Q_{0}=10 \%}$} & \multicolumn{2}{l|}{$P_{f \Delta Q / Q_{0}=20 \%}$} \\
\hline & & $m s^{-1}$ & {$[\mathrm{MW}]$} & $k$ & {$[\mathrm{MW}]$} & $k$ & {$[\mathrm{MW}]$} & $k$ \\
\hline \multirow{3}{*}{$C_{D}=0$} & 0.673 & 1.65 & 15.5 & & 9.2 & & 13.3 & \\
& 0.478 & 3.27 & 55.5 & 1.8 & 29.7 & 1.8 & 44.1 & 1.8 \\
& 0.338 & 6.55 & 191.7 & & 109.3 & & 163.6 & \\
\hline \multirow{3}{*}{$C_{D}=0.6$} & 0.673 & 1.65 & 12.4 & & 7.3 & & 10.7 & \\
& 0.478 & 3.27 & 44.4 & 1.8 & 22.8 & 1.1 & 35.2 & 1.4 \\
& 0.338 & 6.55 & 143.7 & & 47.5 & & 89.9 & \\
\hline \multirow{3}{*}{$C_{D}=1.2$} & 0.673 & 1.65 & 10.4 & & 6.1 & & 8.9 & \\
& 0.478 & 3.27 & 37.0 & 1.8 & 18.1 & 0.9 & 29.4 & 1.1 \\
& 0.338 & 6.55 & 118.5 & & 30.2 & & 60.5 & \\
\hline
\end{tabular}

support structure drag, and hence peak $C_{P c}$ falls with increasing $L / h$ for all values of $C_{D}$.

\subsection{Available power in a volume flow rate reduction con- strained flow}

In this section, the data from the previous section are further studied in terms of dimensional parameters for a more practical interpretation. The variation of the total farm power output with $F r_{w}$ is shown in table 2.1, in which $P_{f \max }$ represents the peak power output for a particular $F r_{w}$ (with the corresponding undisturbed peak channel velocity, $U_{A}$ ) and support structure drag coefficient, $C_{D}$, and other columns represent the power output with volume flow rate constraints of $10 \%$ and $20 \%$. Also shown is the computed exponent $k$ which describes the variation of maximum power output with the undisturbed peak channel velocity; $P_{f} \propto U_{A}^{k}$.

In figure 2.8a, the peak power output is shown as a function of the peak channel velocity corresponding to the three tidal amplitudes discussed earlier, for different support structure drag coefficients. The variation of maximum power output is almost quadratic with respect to undisturbed peak flow speed (for $P_{f \max }, k \approx 1.8$; see table 2.1) but not exactly so due to sea bed friction (irrespective of the level of structural drag). This follows 


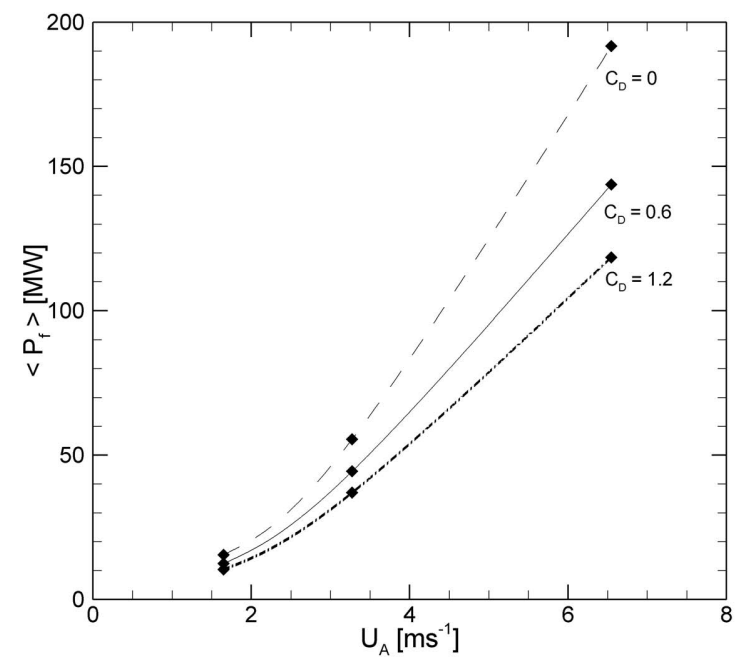

(a) Peak power

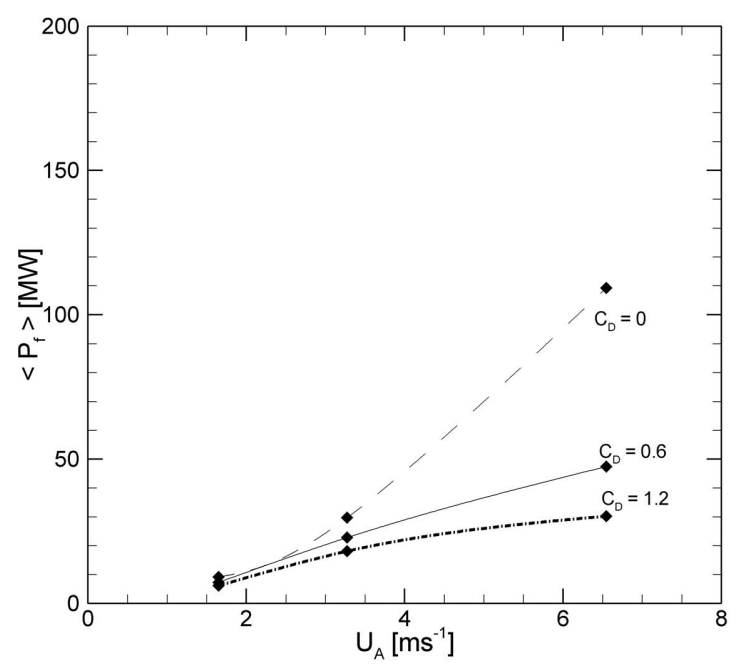

(b) Power with $10 \%$ volume flow rate constraint

Figure 2.8: The influence of maximum undisturbed channel velocity (due to different $F r_{w}$ ) on the time-averaged power output of the tidal farm, $\left\langle P_{f}\right\rangle$, for different support structure drag coefficients. $\left(L / h=125, C_{f}=0.002, \chi=0.2\right)$.

from the energy flux in the tidal wave being proportional to the square of wave amplitude and thus, in the absence of bed friction, to flow speed squared. Similarly in figure $2.8 \mathrm{~b}$, the maximum power output with a $10 \%$ volume flow rate constraint is shown and it can be observed that the behaviour of these curves is quite different from those of peak power. The power-velocity exponents for $C_{D}=0.6$ and 1.2 are markedly reduced to 1.1 and 0.9 respectively and for $\Delta Q / Q_{0}=20 \%$, the exponents are 1.4 and 1.1 for $C_{D}=0.6$ and 1.2 respectively (see table 2.1). Thus, for the channel considered, for a realistic flow rate reduction constraint (10\%) and support structure $\operatorname{drag}\left(C_{D}=1.2, \chi=0.2\right)$, the farm power is sub-linear in $U_{A}$, the undisturbed peak flow velocity, when this is controlled through altering $F r_{w}$. For a fixed geometry channel, different undisturbed peak flow velocities result from different amplitude tidal waves (and thus $F r_{w}$ ). For the channel considered, in the absence of support structures $\left(C_{D}=0\right)$, peak power output for $a=0.5 \mathrm{~m}$ is around $190 M W$ but once the effect of support structures $\left(C_{D}=1.2, \chi=0.2\right)$ and volume flow 
Table 2.2: Farm power output dependency on $\mathrm{L} / \mathrm{h}$

\begin{tabular}{|l|l|l|l|l|l|l|l|l|l|}
\hline$C_{D}$ & $\mathrm{~L} / \mathrm{h}$ & $U_{A}$ & \multicolumn{2}{|c|}{$P_{f \max }$} & \multicolumn{2}{l|}{$P_{f \Delta Q / Q_{0}=10 \%}$} & \multicolumn{2}{l|}{$P_{f \Delta Q / Q_{0}=20 \%}$} \\
\hline & & $m s^{-1}$ & {$[\mathrm{MW}]$} & $k$ & {$[\mathrm{MW}]$} & $k$ & {$[\mathrm{MW}]$} & $k$ \\
\hline \multirow{3}{*}{$C_{D}=0$} & 75 & 1.96 & 13.1 & & 7.4 & & 11.0 & \\
& 125 & 3.27 & 55.5 & 2.7 & 29.7 & 2.7 & 44.1 & 2.7 \\
& 175 & 4.58 & 139.8 & & 79.9 & & 120.1 & \\
\hline \multirow{3}{*}{$C_{D}=0.6$} & 75 & 1.96 & 10.5 & & 6.0 & & 8.9 & \\
& 125 & 3.27 & 44.4 & 2.7 & 22.8 & 2.5 & 35.2 & 2.6 \\
& 175 & 4.58 & 111.7 & & 49.8 & & 84.1 & \\
\hline \multirow{3}{*}{$C_{D}=1.2$} & 75 & 1.96 & 8.8 & & 5.0 & & 7.4 & \\
& 125 & 3.27 & 37.0 & 2.7 & 18.1 & 2.3 & 29.4 & 2.5 \\
& 175 & 4.58 & 93.1 & & 36.0 & & 64.7 & \\
\hline
\end{tabular}

rate constraint (10\%) are considered, the power output drops to $30 \mathrm{MW}$ which is more than an $80 \%$ reduction in the realizable power.

The variation of the total power output with $L / h$ is shown in table 2.2. Similar plots are studied for a range of $L / h$ values in dimensional space; see figure 2.9. The variation in peak power output is a little less than cubic in flow speed (due to bed friction), see table 2.2 , when flow speed is controlled through $L / h$. The impact of structural drag is to reduce the power-velocity exponent, but less markedly so than when $F r_{w}$ is used to control $U_{A}$. For realistic flow rate constraint (10\%), and structural drag $\left(C_{D}=1.2, \chi=0.2\right)$, the exponent is reduced to being a little more than quadratic.

\subsection{Influence of support structure drag coefficient}

In the previous section, the impact of imposing a volume flow rate constraint in the channel was observed to be significant. Hence, in this section, a $10 \%$ volume flow rate reduction constraint is imposed, and the influence of the support structure drag coefficient on $C_{P c}$ is studied. In figure $2.8 \mathrm{~b}$, it was observed that the power output was higher for higher flow speed (lower $F r_{w}$ ) and increases less than quadratically with flow speed. However, once non-dimensionalized, the power coefficient, $C_{P c}$, was observed to decrease with increas- 


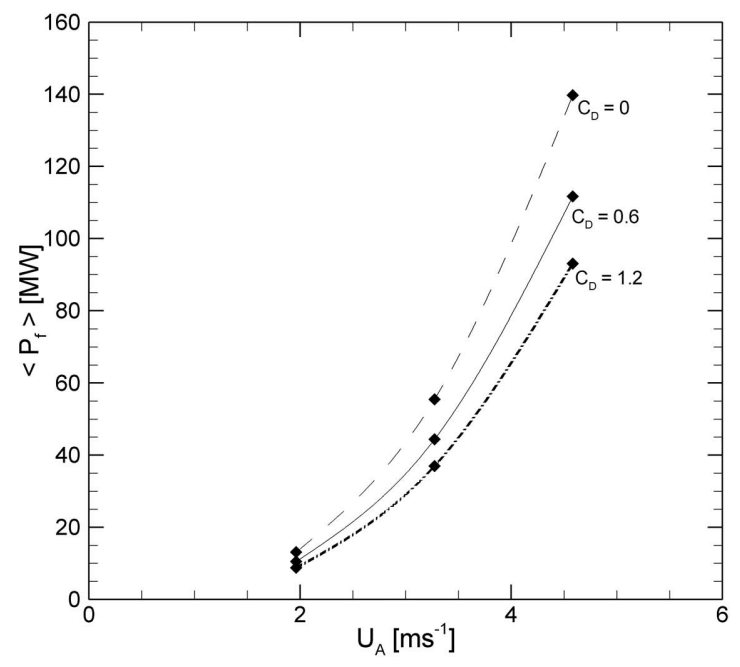

(a) Peak power

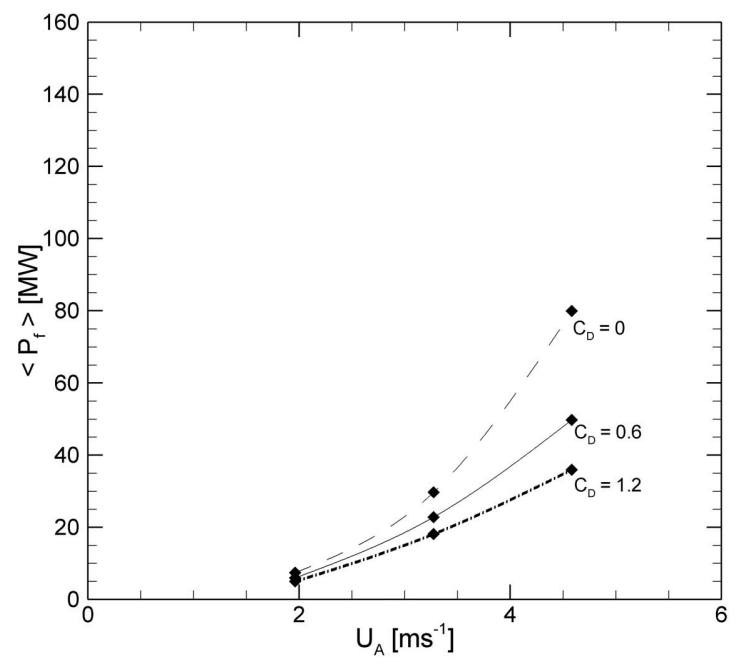

(b) Power with $10 \%$ volume flow rate constraint

Figure 2.9: The influence of maximum undisturbed channel velocity (due to different $L / h)$ on the time averaged power output of the tidal farm, $\left\langle P_{f}\right\rangle$, for different support structure drag coefficients. $\left(F r_{w}=0.478, C_{f}=0.002, \chi=0.2\right)$.

ing flow speed (lower $F r_{w}$ ); see figure 2.10. This indicates that the proportion of channel power that can be realised as useful power decreases as flow velocity increases, due to increasing bed friction losses. Also, as the support structure drag coefficient increases, there is a drop in $C_{P c}$ and the drop is more significant for lower $F r_{w}$ due to greater parasitic drag losses at higher flow speeds; see figure 2.10.

The influence of the non-dimensional parameter $L / h$ was studied in a similar manner by plotting $C_{P c}$ against $C_{D}$; see figure 2.11. As $L / h$ is increased (with $F r_{w}$ maintained at 0.478$), C_{P c}$ decreases. This decrease is due to the increased role that bed friction drag plays at higher flow speeds. Also, as the support structure drag coefficient increases, there is a drop in $C_{P c}$ and this drop is more significant for larger $L / h$ as this corresponds to higher flow speeds. 


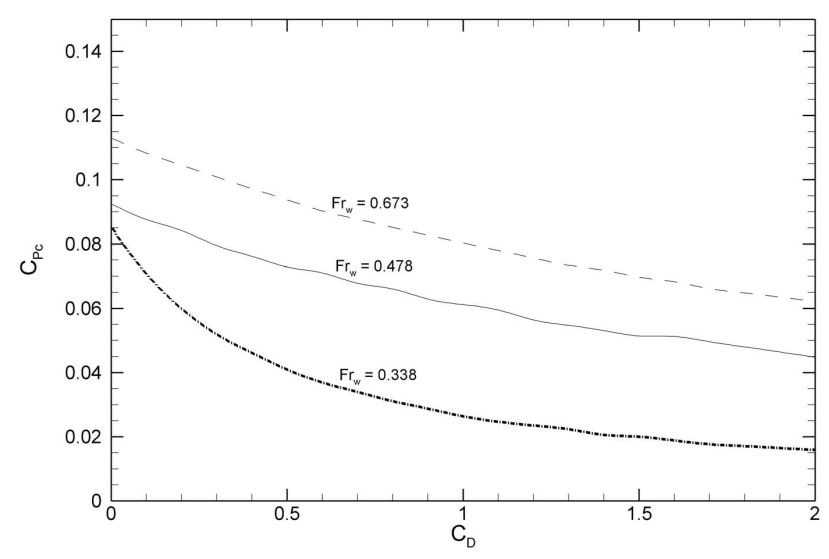

Figure 2.10: The influence of $F r_{w}$ on channel power coefficient, $C_{P c}$, as a function of the drag coefficient of the support structure with a $10 \%$ volume flow rate reduction constraint imposed ( $\left.\chi=0.2, L / h=125, C_{f}=0.002\right)$.

\subsection{Conclusions}

The influence of rotor thrust, support structure drag and seabed friction on the tidal farm power output have been analyzed in this chapter. At the device scale, it was observed that the hydrodynamic efficiency, $\eta$, of a turbine drops in the presence of its support structure. This drop is even greater at higher flow velocities due to the higher drag on the support structures. At the farm scale, the power output was studied for different number of turbines installed in a channel with different support structures, using a one-dimensional channel model. The maximum realizable power output decreased with an increase in the support structure drag coefficient due to increased total flow resistance.

The influence of channel characteristics on the farm power and thrust were studied with the non-dimensional parameters, $F r_{w}=\omega L / \sqrt{g a}$ and $L / h$. For various $F r_{w}$, the power output from the farm varied a little less than quadratically with channel undisturbed peak flow speed, $U_{A}$, due to the effects of seabed friction. The variation in power output for different $L / h$ was observed to be a little less than cubic. Support structure drag had a significant influence on reducing the maximum realizable power and across a range of 


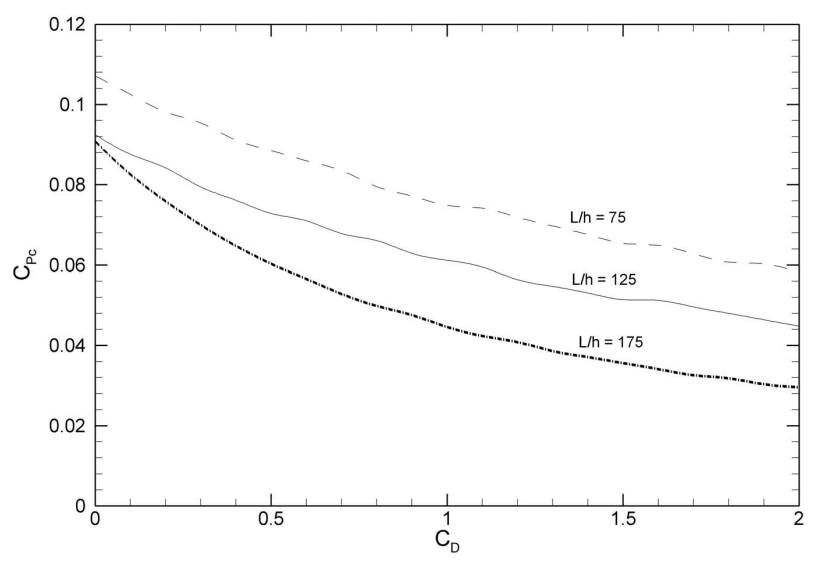

Figure 2.11: The influence of $L / h$ on channel power coefficient, $C_{P c}$, as a function of the drag coefficient of the support structure with a $10 \%$ volume flow rate reduction constraint imposed $\left(\chi=0.2, F r_{w}=0.478, C_{f}=0.002\right)$.

channel characteristics, $F r_{w}$ and $L / h$, reasonable levels of support structure drag reduced the maximum achievable power by up to $40 \%$.

A potential installation of a large number of turbines can significantly decrease the channel flow rate which may be undesirable for environmental impact. Hence a flow rate reduction constraint was imposed such that the peak channel flow rate does not decrease by more than $10 \%$. With this constraint, the realizable farm power varied approximately linearly with the undisturbed channel flow velocity, $U_{A}$, when altering $F r_{w}$ and a little more than quadratic when altering $L / h$. The order $(\mathrm{k})$ of these power output variations with $U_{A}$ is lower relative to the case without any flow rate constraint, indicating that the realizable power with flow rate constraint imposed is lower than the expected power without any constraints. Constraining the permissible flow rate reduction to $10 \%$ renders the structural drag to be even more important, reducing realizable power by up to $70 \%$ relative to the case without support structure drag.

The turbine force coefficients used in this chapter are approximate and computational modelling of a turbine at device scale is needed for a better estimate of force coefficients 
which will be discussed in later chapters. 


\section{Chapter 3}

\section{Numerical Methods}

The impact of different support structure shapes on the turbine efficiency and the overall farm power output was discussed in the previous chapter. It was observed that low drag support structures can significantly increase the overall power output from the farm. A possible low drag support structure shape would be an ellipse but the interaction of the flow from the turbine rotor with the support structure needs to be analyzed, for both circular and elliptical cylinders, to confirm this hypothesis. Hence the focus was shifted from farm scale to device scale, to study the interaction effects between the rotor and its support structure.

The farm-scale analysis was undertaken using an inviscid model but in studying the interaction between a turbine rotor and its support structure, the viscous effects play a significant role. Therefore, Computational Fluid Dynamics (CFD) was used, which is an effective tool for studying the viscous effects. The governing equations and numerical methods used for modelling the flow past a turbine system will be discussed in this chapter. 


\subsection{Governing equations}

The principle of modelling the fluid flow computationally is to predict the transport of different scalar properties, such as velocity, through the domain. The governing equation for the transport of an arbitrary scalar property, $\phi$, is

$$
\frac{\partial \rho \phi}{\partial t}+\nabla \cdot(\rho U \phi)=\nabla \cdot\left(\rho \Gamma_{\phi} \nabla \phi\right)+S_{\phi}(\phi)
$$

where $\phi$ is the fluid density, $t$ is time and $U$ is the flow velocity.

The first two terms on the left hand side are the temporal derivative and the convective term respectively. The first term on the right is the diffusion term, $\Gamma_{\phi}$ is the diffusivity, and the second term is the source term. This is a second order equation as the diffusion term includes a second order derivative of $\phi$.

Obtaining an analytical closed-form solution for equation 3.1 is not possible in most of the cases and therefore numerical methods are used. The basis of numerical methods is to discretize each term of the transport equation to obtain the solution. To ensure the accuracy of the solution, the order of the discretization scheme used, must be at least of the order of the equation (Jasak, 1996).

To understand discretization, consider an arbitrary first order differential equation:

$$
\frac{\partial \phi}{\partial \eta}=k
$$

Using Taylor's series expansion,

$$
\phi_{\eta}=\phi_{\eta-1}+\frac{\partial \phi}{\partial \eta} \Delta \eta+\mathcal{O}\left((\Delta \eta)^{2}\right)
$$

The terms of order two and higher are neglected in this expansion and therefore when this 
expansion is used in equation 3.2, it is a second order discretization resulting in

$$
\phi_{\eta}=\phi_{\eta-1}+k \Delta \eta
$$

Hence the order of discretization depends on the error term neglected in the expansion. A higher order discretization can lead to higher accuracy but increases the computational cost and could also lead to instabilities (Versteeg and Malalasekera, 2007).

\subsection{Discretization of the transport equation}

To solve the transport equation, the entire domain is divided into much smaller control volumes with the equations being solved in each of them. In the Finite Volume Method, each term of the transport equation is integrated through the control volume $(V)$ before employing the discretization procedure as shown in equation 3.5. This is the basis of Finite Volume Method which will be used throughout this work.

$$
\frac{\partial}{\partial t} \int_{V} \rho \phi d V+\int_{V} \nabla \cdot(\rho U \phi) d V=\int_{V} \nabla \cdot\left(\rho \Gamma_{\phi} \nabla \phi\right) d V+\int_{V} S_{\phi}(\phi) d V
$$

Discretization of each term is discussed separately in the next few sections. 


\subsubsection{Spatial Discretization}

\subsubsection{Convection term}

Using the Gauss theorem, the convection term can be rewritten as (Jasak, 1996)

$$
\int_{V} \nabla \cdot(\rho U \phi) d V=\sum_{f} S \cdot(\rho U \phi)_{f}=\sum_{f} F \phi_{f}
$$

where $S$ is the area vector of the control volume face and $F=S \cdot(\rho U)_{f}$, represents the mass flux through the faces; see figure 3.1. The value at the control volume face is calculated by interpolating the values from the neighbouring control volume cell centres. The interpolation schemes commonly used are of first or second order because higher order schemes have a high computational cost associated with them, and can also be quite unstable (Versteeg and Malalasekera, 2007).

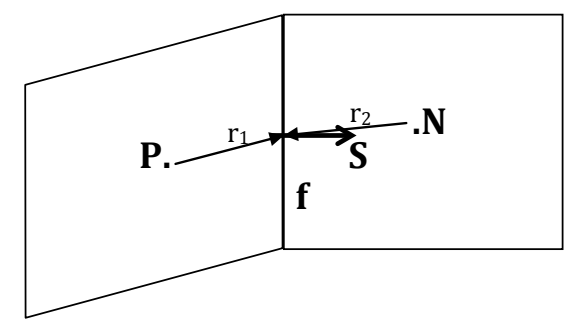

Figure 3.1: A simple representation of neighbouring cells, $\mathrm{P}$ and N, in 2D. $\mathrm{S}$ is an area normal of cell P. $r_{1}$ and $r_{2}$ are the distances from cell centres to face (f) centre.

\subsubsection{First order schemes}

\section{Upwind Scheme}

In the Upwind scheme (UD), the value of a scalar property at the cell face is assumed to be the same as that at the upwind cell centre. Therefore in a first order scheme, the variation 
of $\phi$ between the cell face and the cell centre is neglected; see figure 3.1.

$$
\phi_{f}= \begin{cases}\phi_{P}, & F \geq 0 \\ \phi_{N}, & F \leq 0\end{cases}
$$

In case of a large variation in a scalar property across a cell, Upwind scheme would not be able to predict the variation. Hence Upwind scheme is known to be highly dependent on the mesh and too diffusive in the case of a slightly lower mesh quality.

\subsubsection{Second order schemes}

\section{Second Order Linear Upwind scheme}

Second Order Linear Upwind scheme is similar to Upwind scheme wherein the properties at the face are derived from the properties at the upwind cell centre. As this is a second order scheme, error scales with $\nabla^{2} \phi$, an additional term $\nabla \phi$ is included in the discretization which improves the accuracy of the solution; see figure 3.1.

$$
\phi_{f}= \begin{cases}\phi_{P}+r_{1}(\nabla \phi)_{P}, & F \geq 0 \\ \phi_{N}+r_{2}(\nabla \phi)_{N}, & F \leq 0\end{cases}
$$

Although Second Order Linear Upwind scheme can be more accurate than Upwind scheme, it is known to produce spurious oscillations in the solution in some cases (Versteeg and Malalasekera, 2007). 


\section{Central difference scheme}

In a central difference scheme (CDS), a linear variation of the scalar property is assumed between two neighbouring cells and the interpolation at the face is given by (Jasak, 1996)

$$
\phi_{f}=f_{x} \phi_{P}+\left(1-f_{x}\right) \phi_{N}
$$

where $f_{x}$ is the weighting factor given by

$$
f_{x}=\frac{\overline{f N}}{\overline{P N}}
$$

All the distances are measured orthogonal to the cell face.

A key difference between the central difference scheme and both the upwind difference schemes discussed previously is that in the case of CDS, the direction of the flow is not considered in the discretization procedure. This is the main shortcoming of this scheme as this causes unphysical oscillations in the solution as shown by Patankar (1981), and therefore affects the boundedness of the solution.

\section{LimitedLinear scheme}

As discussed earlier, although second order schemes improve the accuracy of the the solution by limiting numerical diffusion, these schemes lead to oscillations in the solution. To overcome this limitation, a new class of schemes were proposed, known as Total Variation Diminishing (TVD) schemes (Sweby, 1984).

The basis of these schemes is to introduce some upwinding into the solution to mitigate the spurious oscillations. Hence the cell face properties are interpolated by using a combination of first order Upwind scheme (UD) and a higher second order scheme (HO) 
given by (Jasak, 1996)

$$
\phi_{f}=(\phi)_{U D}+\Psi(r)\left[(\phi)_{H O}-(\phi)_{U D}\right]
$$

where $\Psi(r)$ is the flux limiter which is a function of $r . r$ is dependent on the properties of the neighbouring cell centres given by

$$
r=\frac{\phi_{P}-\phi_{W}}{\phi_{N}-\phi_{W}}
$$

where $\phi_{W}$ is the value at the cell centre to the west (left) of $\mathrm{P}$ such that $\mathrm{W}, \mathrm{P}$ and $\mathrm{N}$ are three consecutive cells. It can be noticed that at $\Psi(r)=0$, this scheme turns into a first order upwind scheme and at $\Psi(r)=1$, the scheme is a purely higher order scheme.

Sweby (1984) showed that for a second order TVD scheme to be bounded without oscillations, the variation of the flux limiter, $\Psi(r)$ with $r$, has to lie in the shaded region shown in figure 3.2 .

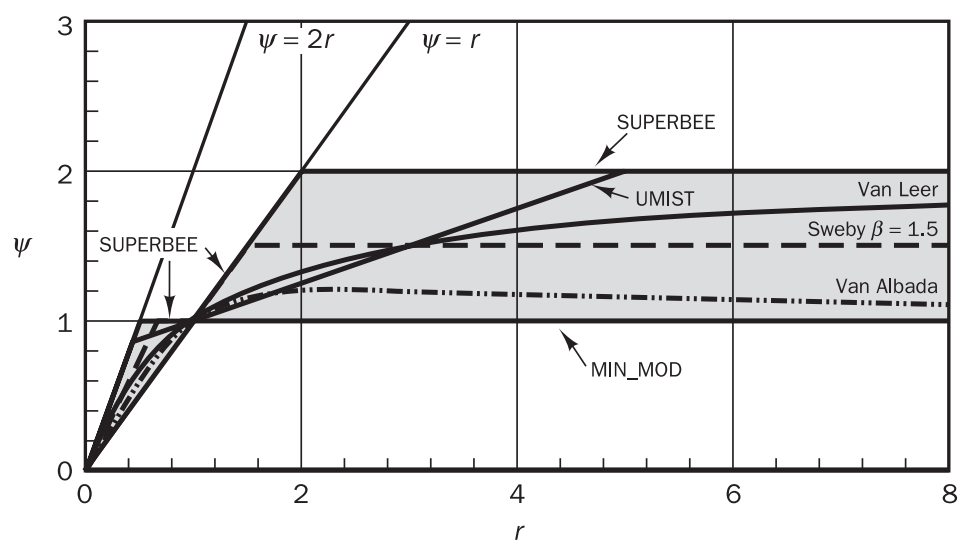

Figure 3.2: Variation of flux limiter $\Psi(r)$ with $r$ for various second order TVD schemes (Versteeg and Malalasekera, 2007).

LimitedLinear (OpenFOAM, 2015) is one of the schemes under the class of TVD schemes. In this method, the higher order scheme used is the central differencing scheme 
with the flux limiter function given by

$$
\Psi(r)= \begin{cases}2 r, & r \leq 0.5 \\ 1, & r \geq 0.5\end{cases}
$$

which lies on the border of the shaded region shown in figure 3.2.

\subsubsection{Diffusion term}

Using Gauss theorem, the diffusion term can be rewritten in the same manner as the convection term (Jasak, 1996).

$$
\int_{V} \nabla \cdot\left(\rho \Gamma_{\phi} \nabla \phi\right) d V=\sum_{f} S \cdot\left(\rho \Gamma_{\phi} \nabla \phi\right)_{f}=\sum_{f}\left(\rho \Gamma_{\phi}\right)_{f} S \cdot(\nabla \phi)_{f}
$$

Diffusion is a process which happens in all the directions unlike convection which depends mainly on the direction of the flow. Hence Upwinding is not necessary in the discretization procedure for the diffusion term. A second order scheme is usually used for the discretization of this term. The gradient term, $\nabla \phi$ is calculated from the properties at the neighbouring cell centres. The Central differencing scheme (Jasak, 1996) is used for the discretization of this term throughout this work.

$$
\begin{aligned}
& \phi_{P}=\phi_{f}-r_{1}(\nabla \phi)_{f}+\mathcal{O}\left(\nabla^{2} \phi\right) \\
& \phi_{N}=\phi_{f}+r_{2}(\nabla \phi)_{f}+\mathcal{O}\left(\nabla^{2} \phi\right)
\end{aligned}
$$

From equations 3.15 and 3.16 ,

$$
(\nabla \phi)_{f}=\frac{\phi_{N}-\phi_{P}}{\overline{P N}}
$$




\subsubsection{Temporal Discretization}

Having discussed the spatial discretization in the previous section, this section describes the temporal discretization and two of the commonly used schemes - Euler implicit scheme and second order backward time differencing scheme.

Consider the scalar transport equation with the time integration:

$$
\int_{t}^{t+\Delta t}\left[\frac{\partial}{\partial t} \int_{V} \rho \phi d V+\int_{V} \nabla \cdot(\rho U \phi) d V\right] d t=\int_{t}^{t+\Delta t}\left[\int_{V} \nabla \cdot\left(\rho \Gamma_{\phi} \nabla \phi\right) d V+\int_{V} S_{\phi}(\phi) d V\right] d t
$$

Rewriting the convection and diffusion terms in terms of the face fluxes from the previous sections,

$$
\int_{t}^{t+\Delta t}\left[\frac{\partial}{\partial t} \int_{V} \rho \phi d V+\sum_{f} F \phi_{f}\right] d t=\int_{t}^{t+\Delta t}\left[\sum_{f}\left(\rho \Gamma_{\phi}\right)_{f} S \cdot(\nabla \phi)_{f}+\int_{V} S_{\phi}(\phi) d V\right] d t
$$

For the temporal discretization of convection and diffusion terms, although $\phi_{f}$ and $(\nabla \phi)_{f}$ vary in time, traditionally their variation in time has been neglected in most of the methods (Patankar, 1981). Instead either the known value from the previous time step or the unknown value at the current time step are used in the discretization (Jasak, 1996).

$$
\int_{t}^{t+\Delta t} \phi(t) d t=\left\{\begin{array}{l}
\phi^{o} \Delta t, \text { explicit scheme } \\
\phi^{n} \Delta t, \text { implicit scheme }
\end{array}\right.
$$

where $\phi^{o}$ is the value at the previous time step which is known, $\phi^{n}$ is the value at the current time step which is to be determined and $\Delta t$ is the time-step. A similar procedure is employed for the diffusion term.

Hence in an explicit scheme, the equations are solved in an explicit manner where $\phi$ at 
the new time step is calculated from only the values at the previous time step whereas in an implicit scheme, $\phi$ at the new time step is calculated by solving a set of simultaneous equations. The time step in an explicit scheme is highly dependent on the the size of the mesh elements, and the scheme is very unstable for general purpose flow calculations (Versteeg and Malalasekera, 2007). The implicit scheme with better coupling between the equations, is a stable scheme and was used in this study.

The integrals in the temporal derivative term of equation 3.19 can be interchanged to obtain

$$
\int_{t}^{t+\Delta t}\left[\frac{\partial}{\partial t} \int_{V} \rho \phi d V\right] d t=\int_{V}\left[\int_{t}^{t+\Delta t} \frac{\partial}{\partial t}(\rho \phi) d t\right] d V
$$

The order of the implicit scheme depends on the discretization of the temporal derivative in equation 3.21. The two commonly used schemes, first order and second order, are described in the next section.

\subsubsection{Euler Implicit scheme}

This is the first order implicit scheme and the discretization of the temporal term is given by

$$
\frac{\partial}{\partial t}(\rho \phi)=\frac{\rho \phi^{n}-\rho \phi^{o}}{\Delta t}
$$

This is a bounded scheme with no limitation on the time step but a higher time step might compromise the accuracy of the solution due to its first order accuracy.

\subsubsection{Backward time difference scheme}

This is a second order implicit scheme and the discretization of the temporal term involves the $\phi$ values from two previous time steps. Using Taylor series expansion,

$$
\phi(t)=\phi^{o}=\phi^{n}-\frac{\partial \phi}{\partial t} \Delta t+\frac{1}{2} \frac{\partial^{2} \phi}{\partial t^{2}} \Delta t^{2}+\mathcal{O}\left(\Delta t^{3}\right)
$$


If the temporal derivative, $\frac{\partial \phi}{\partial t}$, is derived from the above equation, the error term will still scale with only $\Delta t$ and not $(\Delta t)^{2}$ as required for a second order scheme.

To achieve this, the value at time $(t-\Delta t), \phi^{o o}$, is also considered.

$$
\phi(t-\Delta t)=\phi^{o o}=\phi^{n}-2 \frac{\partial \phi}{\partial t} \Delta t+2 \frac{\partial^{2} \phi}{\partial t^{2}} \Delta t^{2}+\mathcal{O}\left(\Delta t^{3}\right)
$$

From equations 3.23 and 3.24, the temporal derivative of second order is derived as (Jasak, 1996)

$$
\frac{\partial \phi}{\partial t}=\frac{\frac{3}{2} \phi^{n}-2 \phi^{o}+\frac{1}{2} \phi^{o o}}{\Delta t}
$$

Similar to the second order spatial discretization schemes, the backward time difference scheme is also not bounded but more accurate than the first order Euler implicit scheme. Hence the backward time difference scheme is used for the current simulations.

\subsection{Turbulence Modelling}

Tidal flow is a turbulent flow, and the presence of turbines leads to more turbulence in the flow. To model the turbulence in a tidal channel, Navier-Stokes equations must be considered. The Navier-Stokes equations are obtained by substituting each component of velocity as the scalar property in the transport equation. The flow in a tidal channel may be treated as an incompressible flow and this study is confined to incompressible Navier-Stokes equations. The Navier-Stokes equations, written in tensorial notation, are respectively (Wilcox, 2004) :

$$
\begin{gathered}
\frac{\partial u_{i}}{\partial x_{i}}=0 \\
\rho \frac{\partial u_{i}}{\partial t}+\rho u_{j} \frac{\partial u_{i}}{\partial x_{j}}=-\frac{\partial p}{\partial x_{i}}+\frac{\partial T_{j i}}{\partial x_{j}}
\end{gathered}
$$


The variables $u, x, t, p$ and $\rho$ are velocity, position, time, pressure and density respectively. In the momentum equation, the terms on the left hand side represent the unsteady and convective acceleration. The pressure gradient term on the right hand side acts against the direction of acceleration and is thus represented by a negative sign. The last term on the right hand side, the viscous stress tensor (T), is defined by (Wilcox, 2004)

$$
T_{i j}=2 \mu s_{i j}
$$

where $\mu$ is molecular viscosity and $s_{i j}$ is the strain rate tensor,

$$
s_{i j}=\frac{1}{2}\left(\frac{\partial u_{i}}{\partial x_{j}}+\frac{\partial u_{j}}{\partial x_{i}}\right)
$$

Different strategies have been proposed to model turbulence. Some of the popular strategies are Large Eddy Simulation (LES), Reynolds Averaged Navier-Stokes model (RANS) and Reynolds Stress Model (RSM). Among these, LES models have the highest computational cost followed by RSM and finally RANS. The Reynolds number (Re), based on the turbine diameter, of tidal flows is much higher than $1 \times 10^{6}$ and to use LES at this Re is computationally infeasible. Both RANS and RSM are good alternatives to LES. Among these, McNaughton (2013) has shown that RANS modelling produces similar results to RSM for turbine simulations, at a cheaper computational cost. Hence RANS modelling was used throughout this study.

The basis of RANS modelling is the Reynolds decomposition wherein the flow variables are divided into a mean component and a fluctuating component (Wilcox, 2004).

$$
u_{i}=U_{i}+u_{i}^{\prime}
$$


where $U_{i}$ is the mean component and $u_{i}^{\prime}$ is the fluctuating component. These components obey certain rules :

$$
\begin{gathered}
\overline{U_{i}}=U_{i} \\
\overline{u_{i}^{\prime}}=0 \\
\overline{u_{i} u_{j}}=\overline{\left(U_{i}+u_{i}^{\prime}\right)\left(U_{j}+u_{j}^{\prime}\right)}=U_{i} U_{j}+\overline{u_{i}^{\prime} u_{j}^{\prime}}
\end{gathered}
$$

Substituting these into equation 3.27, yields the RANS equations (Wilcox, 2004)

$$
\begin{gathered}
\frac{\partial U_{i}}{\partial x_{i}}=0 \\
\rho \frac{\partial U_{i}}{\partial t}+\rho U_{j} \frac{\partial U_{i}}{\partial x_{j}}=-\frac{\partial P}{\partial x_{i}}+\frac{\partial}{\partial x_{j}}\left(2 \mu S_{j i}-\rho \overline{u_{j}^{\prime} u_{i}^{\prime}}\right)
\end{gathered}
$$

The quantity $-\rho \overline{u_{i}^{\prime} u_{j}^{\prime}}$ is known as the Reynolds Stress tensor and is usually denoted by $\rho \tau_{i j}$, so that the specific Reynolds Stress tensor $\left(\tau_{i j}\right)$ is given by

$$
\tau_{i j}=-\overline{u_{i}^{\prime} u_{j}^{\prime}}
$$

In the RANS equations, there are ten unknowns (four mean components and six Reynolds Stress components) but only four equations. To close these equations, the 'Boussinesq approximation' is used wherein the Reynolds Stresses are linked to the mean components of the flow (Tennekes and Lumley, 1972):

$$
\tau_{i j}=\nu_{t}\left(\frac{\partial U_{i}}{\partial x_{j}}+\frac{\partial U_{j}}{\partial x_{i}}\right)-\frac{2}{3} \rho k \delta_{i j}
$$

where $\nu_{t}$ is the eddy viscosity and $k$ is the specific kinetic energy of velocity fluctuations 
given by

$$
k=\frac{1}{2} \overline{u_{i} u_{i}}
$$

The presence of the Kronecker delta $\left(\delta_{i j}\right)$ is necessary to account for the correct normal Reynolds Stresses.

The Boussinesq approximation is the basis of RANS turbulence modelling. The RANS equations are closed by further solving for $k$ and $\nu_{t}$. This is done by solving zero, one or two equations based on the model.

The zero equation model is an algebraic model which does not involve solution of additional equations, but instead the values are calculated from the existing variables. Although this model is relatively simple, it is not known to predict the flow accurately in complex cases as it does not account for the history effects on turbulence (Wilcox, 2004).

One equation turbulence models usually solve the transport equation for kinetic energy. The original one-equation model is the Prandtl's one-equation model (Wilcox, 2004) but one of the more commonly used model is the Spalart and Allmaras (1992) model. Although these models are known to predict the flow in certain cases with less flow separation, they are unable to predict highly separated flows that occur past bluff bodies like a cylinder.

Two-equation turbulence models solve an additional transport equation along with the kinetic energy. Although there have been many models proposed regarding this second equation, there is no model which applies universally to all the turbulent flows. In external flows, two models are particularly popular, the $k-\varepsilon$ model and the $k-\omega$ model. In the $k-\varepsilon$ model, the second transport equation solved is for turbulent dissipation $(\varepsilon)$. This model has the advantage of freestream independence wherein the solution is not affected significantly by the inlet turbulence parameters but the major shortcoming of this model is in predicting the flow in the near-wall region. There have been many variants of this 
model, Jones and Launder (1972), Launder and Sharma (1974), but none have been able to accurately predict the near-wall gradients.

The $k-\omega$ model was later formulated by Wilcox (1988) which improved the prediction of near wall region. In this model, the second transport equation solved is for the specific turbulent dissipation $(\omega)$. The transport equations solved in this model are presented below:

$$
\begin{gathered}
\frac{\partial k}{\partial t}+U_{j} \frac{\partial k}{\partial x_{j}}=\tau_{i j} \frac{\partial U_{i}}{\partial x_{j}}-\beta^{*} k \omega+\frac{\partial}{\partial x_{j}}\left[\left(\nu+\sigma^{*} \nu_{t}\right) \frac{\partial k}{\partial x_{j}}\right] \\
\frac{\partial \omega}{\partial t}+U_{j} \frac{\partial \omega}{\partial x_{j}}=\frac{\alpha}{\nu_{t}} \tau_{i j} \frac{\partial U_{i}}{\partial x_{j}}-\beta \omega^{2}+\frac{\sigma_{d}}{\omega} \frac{\partial k}{\partial x_{j}} \frac{\partial \omega}{\partial x_{j}}+\frac{\partial}{\partial x_{j}}\left[\left(\nu+\sigma \nu_{t}\right) \frac{\partial \omega}{\partial x_{j}}\right]
\end{gathered}
$$

where $\alpha, \beta, \beta^{*}, \sigma, \sigma^{*}$ and $\sigma_{d}$ are closure coefficients presented in Wilcox (1988). Although the $k-\omega$ model provides a robust and accurate formulation in the near-wall region, it is not as freestream independent as the $k-\varepsilon$ model.

To overcome the freestream dependence of the $k-\omega$ model, a new Baseline (BSL) model was presented by Menter (1993) wherein the Wilcox (1988) model was used in the inner wall region, but gradually changes to the $k-\varepsilon$ model (Launder and Sharma, 1974) in the free shear layers. In deriving the transport equations for this BSL model, the $\varepsilon$ equation of the $k-\varepsilon$ model is written in terms of $\omega$ and then the two models are combined using a blending function. The blending function models the transition between $k-\omega$ and $k-\varepsilon$ models in the wake of the boundary layer. Hence the transport equation for the kinetic energy remains the same as equation 3.39 but the $\omega$ equation is modified:

$$
\frac{\partial \omega}{\partial t}+U_{j} \frac{\partial \omega}{\partial x_{j}}=\frac{\alpha_{s}}{\nu_{t}} \tau_{i j} \frac{\partial U_{i}}{\partial x_{j}}-\beta_{s} \omega^{2}+2\left(1-F_{1}\right) \frac{\sigma_{d}}{\omega} \frac{\partial k}{\partial x_{j}} \frac{\partial \omega}{\partial x_{j}}+\frac{\partial}{\partial x_{j}}\left[\left(\nu+\sigma_{s} \nu_{t}\right) \frac{\partial \omega}{\partial x_{j}}\right]
$$


where $F_{1}$ is the blending function with a value of unity in the near-wall region and zero away from the wall. It should be noted that the values of the closure coefficients in this model are different from both the $k-\omega$ and $k-\varepsilon$ models.

The BSL model was further extended in $k-\omega$ SST model (Menter, 1994) to better predict the separation in adverse pressure gradient cases. Menter (1994) realised that the main difference between eddy viscosity models and Reynolds Stress models in predicting the flow separation was due to the fact that the Reynolds Stress model (Launder et al., 1975) accounts for the transport of principal turbulent shear stress $(\tau) . k-\omega$ SST model extends Bradshaw's assumption (Menter, 1994), that the turbulent shear stress in a boundary layer is proportional to the turbulent kinetic energy $\left(\tau=\rho a_{1} k\right)$, by rewriting it as

$$
\tau=\rho \sqrt{\frac{\text { Production }_{k}}{\text { Dissipation }_{k}}} a_{1} k
$$

where $a_{1}$ is a constant. But in the case of adverse pressure gradient flows, the ratio of production to dissipation can be significantly greater than unity and hence the turbulence shear stress is over-predicted. On the other hand, the turbulent shear stress in RANS two equation models is calculated using the Boussinesq approximation, equation 3.37. Hence to limit the turbulent shear stress, the turbulent eddy viscosity, $\nu_{t}$, can be constrained. The $k-\omega$ SST model constrains the turbulent eddy viscosity by

$$
\nu_{t}=\frac{a_{1} k}{\max \left(a_{1} \omega ; \Omega F_{2}\right)}
$$

where $\Omega=\partial u_{i} / \partial x_{j}$ and $F_{2}$ is a function with a value of unity for boundary layer flows and zero for free shear layers (Menter, 1994). This constraint is the basis of the $k-\omega \mathrm{SST}$ model allowing it to better predict separated flows than other two-equation models. Hence $k-\omega$ SST model will be used throughout this study. 


\subsection{Wall Functions}

Modelling the flow near wall regions is complicated as the flow physics change within the turbulent boundary layer. In the inner layer, the flow can be divided into three layers based on the velocity variation with distance from the wall: viscous sublayer, buffer layer and log-law region.

The flow physics is described in the dimensionless form of velocity $\left(u^{+}\right)$and wall distance $\left(y^{+}\right)$:

$$
u_{\tau}=\sqrt{\frac{\tau_{w}}{\rho}}, \quad u^{+}=\frac{u}{u_{\tau}}, \quad y^{+}=\frac{y u_{\tau}}{\nu}
$$

where $\tau_{w}$ is the wall shear stress and $u_{\tau}$ is the friction velocity.

Based on various experimental studies (Tennekes and Lumley, 1972), it was observed that in the viscous sublayer $\left(y^{+}<5\right)$, the velocity varies with wall distance according to $u^{+}=y^{+}$. In the log-law region $\left(y^{+}>25\right)$, this relation is given by:

$$
u^{+}=\frac{1}{\kappa} \ln y^{+}+C^{+}
$$

where $\kappa(=0.41)$ is the von Kármán constant and $C^{+}$is an empirical constant. The buffer layer does not obey any particular relation and this poses a certain problem in resolving the mesh in the boundary layer.

To accurately resolve the boundary layer, two different approaches might be used. In the first method, the first cell centre adjacent to the wall lies in the log-layer and wall functions are used to model the flow in the other regions $\left(y^{+}<25\right)$. In the second approach, the first cell centre adjacent to the wall lies within the viscous sublayer $\left(y^{+}<5\right)$ and the entire boundary layer is resolved. Also, one needs to ensure that there are enough nodes in the boundary layer, which is usually a large number, to capture the flow phenomenon. 
Hence the second approach results in a mesh count significantly larger than the first approach and consequently a higher computation cost. Therefore, in cases where boundary layer separation is not significant, the first approach is usually used.

Both the methods discussed above were employed in the present work. In the case of turbine rotor blades, where there is less flow separation from the airfoil sections, the first approach with wall functions was used. In the case of support structures, where there is significant flow separation, wall functions may not be trusted to produce accurate results and hence the second approach was employed wherein the entire boundary layer is resolved.

\subsection{Simulating Rotor motion}

The flow past a turbine rotor involves complex physics with high computation load. There are two different approaches which were used to simulate the rotor motion, Mesh motion and Multiple Reference Frame (MRF) approach.

\subsubsection{Mesh motion}

In this approach, a coin shaped region is created around the turbine rotor which shares interfaces with the rest of the domain. The turbine rotor motion is simulated by physically rotating this mesh region enclosing the turbine. Hence the rotor mesh rotates at a specified rate while the mesh in the outer domain remains stationary.

As the rotor mesh rotates, the swirl flow in the inner region is propagated along with other flow variables through the interface into the outer domain. The interface data transfer is done using Arbitrary Mesh Interface mechanism (OpenFOAM, 2015).

Arbitrary Mesh Interface (AMI) is based on the algorithm presented by Farrell and 
Maddison (2011). In this approach, there are separate meshes for rotating and stationary regions which are coupled at their interfaces. This approach works even for nonconformal interfaces which is an improvement over the older approaches. In the case of non-conformal meshes, two or more cell surfaces of one interface might be in contact with a single cell surface on the other interface. Therefore, weight factors are assigned to each of the overlapping cell surfaces defining their contribution as a fraction of the intersecting area (OpenFOAM, 2015).

The mesh count in the rotor region is high and to physically rotate the mesh involves high computational cost but this would be necessary to study the unsteady wake characteristics of the turbine and its influence on the support structure.

\subsubsection{Multiple Reference Frame}

In the Multiple Reference Frame (MRF) approach, to reduce the high computational costs associated with the previous method, multiple reference frames are used for different regions in the domain. A coin shaped region is created around the turbine rotor similar to the previous method, but instead of physically rotating the mesh, the solution in this inner region is obtained by solving the Navier-Stokes equations in a rotating reference frame.

As there is no mesh motion involved in this approach, MRF can be used to solve the simulation in a steady (non-transient) state. This is another major advantage of this approach as it results in almost an order of magnitude reduction in the computational cost.

The domain in this approach consists of two zones - a rotating zone around the rotor where MRF is applied and a stationary outer zone. Using the concept of relative velocity, the relation between the velocity of fluid in the rotating frame of reference $\left(\mathbf{u}_{\mathbf{R}}\right)$ and its 
absolute velocity $\left(\mathbf{u}_{\mathbf{I}}\right)$ in the global stationary frame is given by

$$
\mathbf{u}_{\mathrm{I}}=\mathbf{u}_{\mathbf{R}}+\Omega \times \mathbf{r}
$$

where $\Omega$ is the angular velocity of the rotating region and $r$ is the position vector of a cell within this region.

Using equation 3.45, the momentum equations (steady state) are transformed in the rotating frame of reference (OpenFOAM, 2015)

$$
\nabla \cdot\left(u_{R} \otimes u_{R}\right)+2 \Omega \times u_{R}+\Omega \times(\Omega \times r)=-\nabla(p / \rho)+\nu \nabla \cdot \nabla\left(u_{R}\right)
$$

The momentum equations in the outer stationary region would remain the same as discussed earlier. To ensure that the velocity convected, at the interfaces between the inner and outer regions, is in terms of absolute velocity, equation 3.46 is modified to include absolute velocity $\left(u_{I}\right)$ using equation 3.45 .

$$
\nabla \cdot\left(u_{R} \otimes u_{I}\right)+\Omega \times u_{I}=-\nabla(p / \rho)+\nu \nabla \cdot \nabla\left(u_{I}\right)
$$

A key shortcoming of this approach is that as the fluid leaves the rotating region, the rotating component of the flow is lost and hence the wake is not as physically expected. Nevertheless, MRF is a good approximation for calculating the force and power coefficients of a turbine rotor.

\subsection{Boundary Conditions}

The specification of boundary conditions can be done in different ways - Neumann and Dirichlet. In the Neumann type condition, the gradient of the property is specified at the 
boundary, whereas in the Dirichlet type condition, the property value itself is specified . The different boundary conditions used in this study are discussed below:

\section{Inlet}

The inlet is a boundary condition to define the properties of the flow in the free stream. The velocity and turbulence parameters, $k$ and $\omega$, are defined as Dirichlet type boundary conditions whereas the pressure is defined as zero gradient, Neumann type.

\section{Outlet}

The outlet is the boundary condition used to define the flow leaving the domain. Here, the pressure is defined by the Dirichlet boundary condition whereas the velocity and turbulence parameters are defined by the Neumann type.

\section{Symmetry}

In the symmetry boundary condition, the pressure and tangential velocities are defined by Neumann type whereas the normal velocity components are set to zero.

\section{Wall}

In the wall boundary condition, the pressure is defined by Neumann type while the velocity is defined by the Dirichlet type, with all the components of velocity being zero at the wall. 


\section{Chapter 4}

\section{Model rotor and support structure}

\section{validation}

Before analyzing full-scale rotor-support structure interaction, a model-scale rotor and its support structure is studied in this chapter to validate the computational setup. The numerical methods discussed in the previous chapter will be used to simulate the modelscale turbine system using CFD and then compared with experimental data. One of the more comprehensive experiments on tidal turbines was performed by Bahaj et al. (2007b) for various tip speed ratios of the turbine. Although this study was extensive, the position of the support structure was too far away behind the rotor to capture the interaction effects, as discussed by the authors. Therefore, the validation of a turbine rotor and its support structure interaction, as a whole, was not possible due to the lack of experimental studies. Hence, a building block approach (Versteeg and Malalasekera, 2007) was used.

In a Building block approach, the complexity of a system is reduced by decomposing it into smaller components, and these components are validated. In the present study, the entire turbine system is decomposed into support structure and rotor, and each of them are validated separately. 
The finite-volume solver OpenFOAM (2015) was used for all the simulations. To reduce the run-time of the simulations, they were run on the ARCUS super computer at Oxford.

This chapter starts with the validation of the two support structures considered, circular and elliptical cylinders, followed by the rotor validation. In the last section, a comparison between the two approaches to rotor simulation, Multiple Reference Frame (MRF) and Mesh motion, is discussed.

\subsection{Cylindrical monopile}

A cylindrical monopile is a commonly used support structure for wind turbines and in the conceptual designs of tidal turbines. Therefore, this was the first support structure examined. There have been numerous studies in the past describing the flow past a cylindrical structure, both experimental and computational.

Roshko (1961) studied the flow past a circular cylinder in the Reynolds number range of $10^{6}-10^{7}$, and discussed the change in drag coefficient and Strouhal number at these high Reynolds numbers. Reynolds number is the ratio of inertial to viscous forces within a fluid given by $v L / \nu$, and Strouhal number describes the oscillating flow mechanisms such as the vortex shedding behind a cylinder given by $f L / v$; $v$ is the flow velocity, $f$ is the frequency of vortex shedding, $L$ is the characteristic length and $\nu$ is the kinematic viscosity of the fluid.

Later, Achenbach (1968) conducted experiments on a cylinder in the similar Reynolds number range, and obtained the pressure coefficient and skin friction coefficient along the circumference of the cylinder. More recently, Catalano et al. (2003) has performed numerical simulations on a 3D cylinder using RANS and LES, and compared their results with the experimental results, demonstrating the capability of CFD for studying these type 
of flows.

Due to computational constraints, the RANS method is used in the present work to simulate the flow past a cylinder. Using the same building block approach as before, a 2D section of the cylinder is initially studied to optimise the mesh, and then the 3D cylinder is simulated.

\subsubsection{Cylinder 2D}

The computational cost associated with simulating the 2D cylinder is much less than the 3D cylinder and hence the mesh parameters can be quickly optimised. The domain considered for simulating the flow past a $2 \mathrm{D}$ cylinder is shown in figure 4.1 .

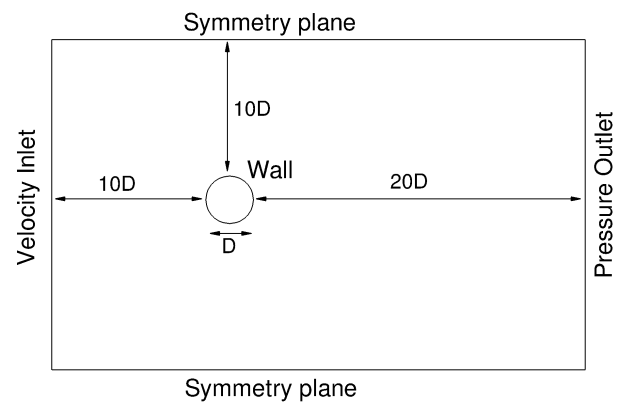

Figure 4.1: Computational domain for simulating flow past a cylinder

The inlet and outlet are designated as velocity inlet and pressure outlet respectively. The sides of the domain are considered symmetry planes. The cylinder itself has the wall boundary condition.

\section{Computational setup}

The flow past a support structure in a tidal channel is in the high Reynolds number turbulent regime. This turbulent regime for the flow past a cylinder starts around $2 \times 10^{6}$. Hence the inlet velocity for this simulation is chosen such that the Reynolds number is $2 \times 10^{6}$. 
The flow past a cylinder results in a highly separated unsteady flow. To model the turbulence in such separated flows, Reynolds Stress Model (RSM) and $k-\omega$ SST model are widely used (Wilcox, 2004). RSM model (Launder et al., 1975) solves seven transport equations whereas $k-\omega$ SST model (Menter, 1994) solves only two transport equations, thus significantly reducing the computational cost and for this reason, $k-\omega$ SST model is used throughout this study.

The spatial discretization was entirely second order, with a second-order backward scheme applied temporally.

The freestream turbulence parameters, $k$ and $\omega$, are calculated based on the inlet velocity $(U)$, turbulence intensity $(I)$ and the turbulent length scale $(l)$ given by

$$
\begin{gathered}
k=\frac{3}{2}(U I)^{2} \\
\epsilon=\frac{C_{\mu}^{\frac{3}{4}} k^{\frac{3}{2}}}{l} \\
\omega=\frac{\epsilon}{k}
\end{gathered}
$$

where $\epsilon$ is the turbulence dissipation rate and $C_{\mu}$ is an empirically calibrated constant in the $k-\omega$ SST model (Menter, 1994).

\section{Blocking strategy}

A structured mesh was created with an 'O'grid blocking strategy. A fine mesh was created close to the cylinder to capture the high gradients in the boundary layer; see figure $4.2 \mathrm{~b}$. The cell immediately adjacent to cylinder surface had dimensions such that wall $y^{+}<3$, thus fully resolving the boundary layer instead of using the wall functions. This is because the standard wall functions are known to produce inaccurate results in highly separated flows. The mesh size is gradually increased away from the cylinder but still sufficiently 


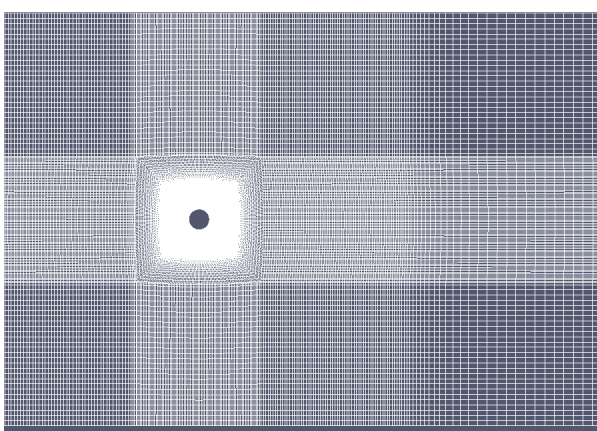

(a) Mesh in the domain

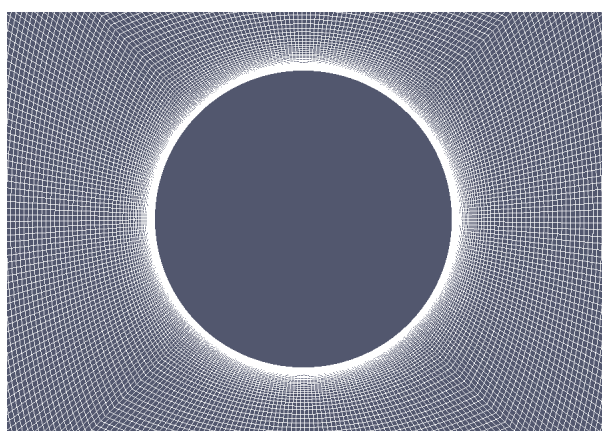

(b) Mesh close to the cylinder

Figure 4.2: Mesh around the 2D cylinder.

fine to capture the wake. Farther away from the cylinder, a relatively coarse mesh is used to reduce the computational cost; see figure 4.2a.

\section{Mesh independence study}

The number of elements on the cylinder surface and the growth ratio of the elements in the radial direction away from the cylinder can affect the predicted flow field around the cylinder, thus influencing the force coefficients. Hence three different meshes were studied with varying number of elements, and the pressure on the surface of the cylinder was analysed; see figure 4.3.

To study the pressure $(p)$ on the surface, it is non-dimensionalised as the pressure coefficient given by

$$
C_{p}=\frac{p-p_{\infty}}{\frac{1}{2} \rho U_{\infty}^{2}}
$$

where $p_{\infty}$ and $U_{\infty}$ are the freestream pressure and velocity respectively.

The pressure coefficient is similar using the medium and fine meshes but there is a significant difference with the coarse mesh; see figure 4.3. The pressure coefficient is similar until the point of flow separation, but here the coarse mesh predicts a higher pressure re- 


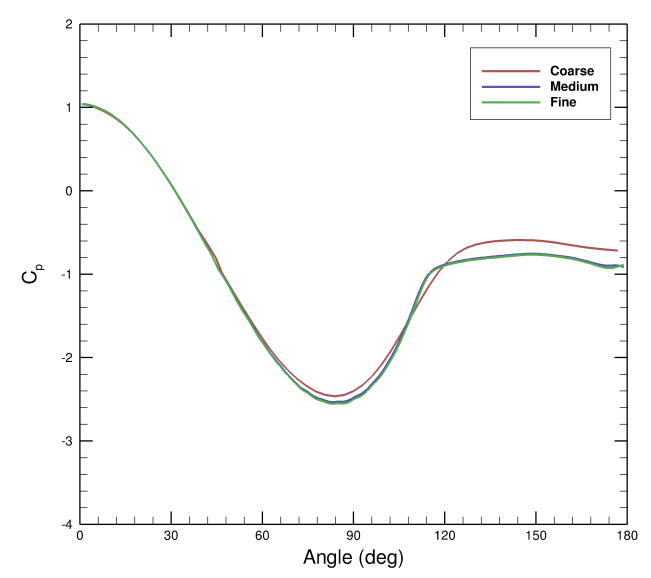

Figure 4.3: Profile of mean pressure coefficient on the cylinder surface using different meshes.

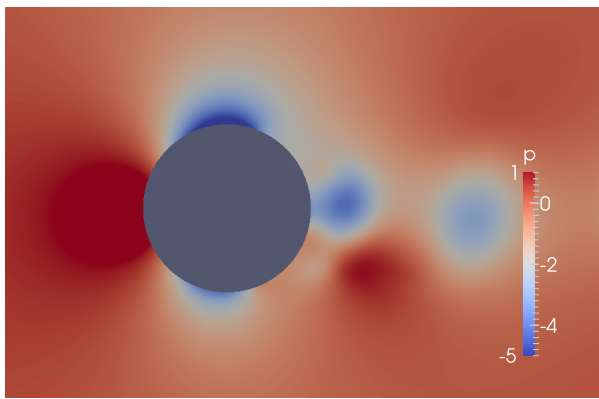

(a) Pressure

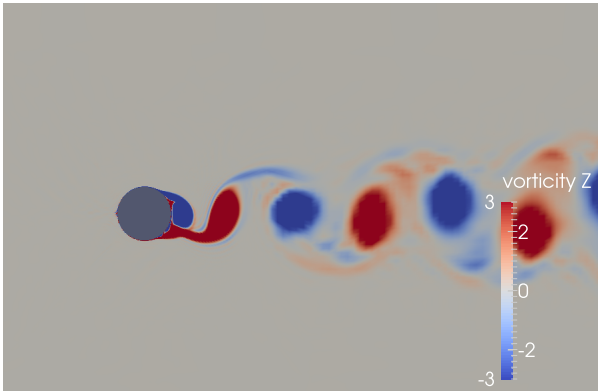

(b) Vorticity (Z)

Figure 4.4: Visualization of pressure (a) and vorticity (b) distributions in unsteady, unidirectional flow past a cylinder at $R e=2 \times 10^{6}$.

covery which would result in a lower prediction of the drag coefficient. Hence the medium mesh was chosen for the rest of the analysis.

The instantaneous pressure around the cylinder using this mesh is shown in figure 4.4. The high pressure stagnation point at the front of the cylinder can be clearly noticed. The vortex street behind the cylinder is shown in figure $4.4 \mathrm{~b}$ where the alternating vortex shedding from the rear of the cylinder surface can be noticed. 


\subsubsection{Cylinder 3D}

The flow physics around a cylinder in $3 \mathrm{D}$ is different from the $2 \mathrm{D}$ simulations due to the three dimensional nature of turbulence in the cylinder wake (Williamson, 1988). To simulate a 3D flow, the spanwise length can be of particular importance to emulate an infinitely long cylinder.

Lei et al. (2001) have shown that 2D CFD simulations were unable to predict the forces accurately and hence used a finite length cylinder for better prediction at a Reynolds number of 1000 . They observed that the $3 \mathrm{D}$ simulation might still produce inaccurate results if the spanwise length chosen is too small. To obtain the minimum spanwise length to predict accurate results, Labbe and Wilson (2007) simulated multiple spanwise lengths of $\pi D / 2, \pi D$ and $2 \pi D$, and demonstrated that a finite spanwise extension of $\pi D$ was sufficient to accurately predict the flow at a Reynolds number of 1000.

Norberg (2003) defined a correlation length based on the near cylinder velocity fluctuations in the outer parts of the separated shear layer, and used this correlation length as the minimum spanwise length of the cylinder required to simulate a 3D flow. This spanwise correlation length is higher at lower Reynolds number and decreases towards the higher Reynolds number regime. Based on Norberg (2003), a spanwise length of 4D was chosen to simulate the high Reynolds number 3D flow in the present work.

As the mesh independence in $2 \mathrm{D}$ was already studied, the mesh in this $3 \mathrm{D}$ simulation is optimised by varying the number of spanwise elements. The number of elements were varied from 10 to 30 in intervals of 10 ; see table 4.1 .

The coarse mesh predicts a higher separation angle and consequently lower drag coefficient. The remaining two meshes predicted similar results and hence 20 spanwise elements were chosen for the study. These results were then compared with the experimental data. 
Table 4.1: Spanwise mesh dependency

\begin{tabular}{|c|c|c|c|}
\hline Spanwise elements & $C_{\text {DMean }}$ & Separation angle & Strouhal Number \\
\hline 10 & 0.56 & $121^{\circ}$ & 0.18 \\
\hline 20 & 0.59 & $117^{\circ}$ & 0.2 \\
\hline 30 & 0.59 & $117^{\circ}$ & 0.2 \\
\hline
\end{tabular}

Warschauer and Leene (1971) have conducted experiments on a 3D cylinder and plotted the pressure coefficients at a Reynolds number of $1.2 \times 10^{6}$. Achenbach (1968) also performed experiments over a range of Reynolds numbers around $10^{6}$, and plotted pressure coefficients at each of these Reynolds numbers. Since the Reynolds number in the present study is $2 \times 10^{6}$, the experimental data at $3.6 \times 10^{6}$ in Achenbach (1968), which is the closest Reynolds number, was chosen for comparison along with Warschauer and Leene (1971).

Along with the experimental results, the CFD simulation performed by Catalano et al. (2003) using Unsteady Reynolds Averaged Navier-Stokes (URANS) at Reynolds number of $1 \times 10^{6}$ was also used for comparison.

The pressure coefficient of the present simulation follows a similar pattern as the experimental values until flow separation. The present work agrees better with the results of Achenbach (1968) at $\operatorname{Re}=3.6 \times 10^{6}$. This might be because the results of Achenbach (1968) were for the flow in a higher turbulent regime which the CFD code simulates, compared to a possible transition regime in the results of Warschauer and Leene (1971) at Re $=1.2 \times 10^{6}$. The other CFD results by Catalano et al. (2003) predicted a higher pressure recovery.

To study the separation on the cylinder, the wall shear stress needs to be analysed. The 


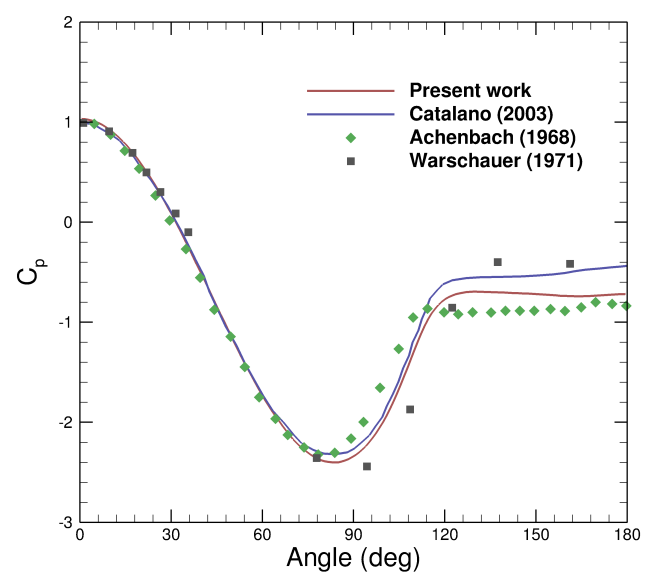

Figure 4.5: Comparison of pressure coefficient; CFD (Present work) at $\operatorname{Re}=2 \times 10^{6}$, CFD (URANS) at $\operatorname{Re}=1 \times 10^{6}$ by Catalano et al. (2003), Experimental at $\operatorname{Re}=3.6 \times 10^{6}$ by Achenbach (1968), Experimental at $\operatorname{Re}=1.2 \times 10^{6}$ by Warschauer and Leene (1971).

wall shear stress $\left(\tau_{w}\right)$ is studied in the non-dimensional form as the skin friction coefficient

$$
C_{f}=\frac{\tau_{w}}{\frac{1}{2} \rho U_{\infty}^{2}}
$$

The wall shear stress is also an indicator of the viscous force on the cylinder but as this is considerably lower than the pressure force, the magnitude of the wall shear stress (skin friction) would not make a large difference to the force coefficients. But the skin friction coefficient is useful in determining the separation point because the skin friction is zero at the point of flow separation.

The skin friction coefficient is over-predicted on the front surface of the cylinder compared to experimental results; see figure 4.6. This is because the turbulence models in CFD assume the entire boundary layer to be turbulent but there might be a laminar boundary layer on the front surface of the cylinder which has a lower skin-friction coefficient. But this does not affect the force coefficients in a large way as discussed earlier. Note that the other CFD study of Catalano et al. (2003) also over predicts the skin friction on the front 


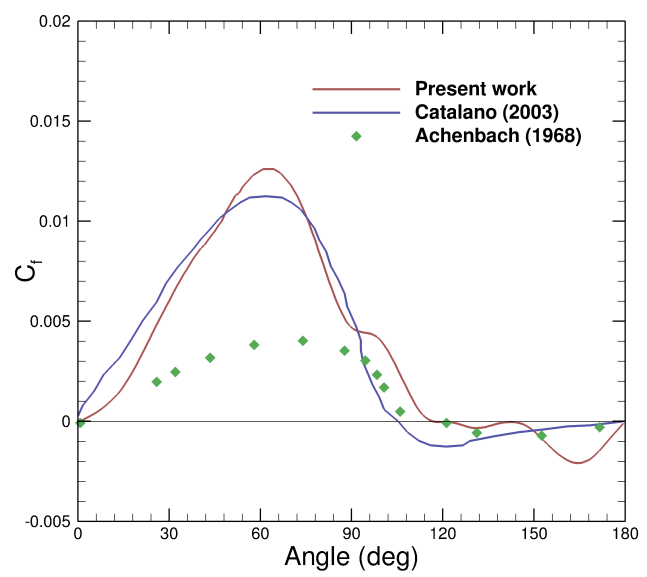

Figure 4.6: Comparison of skin friction coefficient; CFD (Present work) at $\operatorname{Re}=2 \times 10^{6}$, CFD (LES) at $\mathrm{Re}=1 \times 10^{6}$ by Catalano et al. (2003), Experimental at $\mathrm{Re}=3.6 \times 10^{6}$ by Achenbach (1968).

Table 4.2: Comparison with experimental and CFD data

\begin{tabular}{|c|c|c|c|}
\hline & $C_{D \text { Mean }}$ & Separation angle & Strouhal Number \\
\hline Present work & 0.59 & $117^{\circ}$ & 0.2 \\
\hline Experimental: Roshko (1961), Achenbach (1968) & $0.55-0.6$ & $116^{\circ}-120^{\circ}$ & $0.2-0.22$ \\
\hline CFD URANS: Catalano et al. (2003) & 0.4 & - & 0.31 \\
\hline
\end{tabular}

surface of the cylinder.

The separation point on the cylinder is predicted very close to the experimental results while the study by Catalano et al. (2003) predicts a slightly earlier separation.

The comparison of force coefficients is shown in table 4.2. The drag coefficient by Catalano et al. (2003) is slightly lower due to the higher predicted pressure recovery as discussed earlier. All the results of the present study are in the range of experimental data at this Reynolds number. 


\subsection{Elliptical monopile}

An elliptical monopile might be a good alternative to a circular monopile as it has a much lower drag coefficient than a circular cylinder. Also its symmetry is advantageous during the flood and ebb tide, when the flow reverses by $180^{\circ}$.

Although an ellipse is symmetric about its major and minor axes, it is not entirely symmetric about every rotational axis as a circular cylinder. Hence a change in the direction of the incoming flow, angle of attack, might have an adverse effect on the structure which will be discussed in this section.

To lower the computational cost, the influence of angle of attack on the drag and lift of an ellipse is studied on a 2D section of an ellipse.

\subsubsection{Ellipse 2D}

The domain used for this simulation was the same as that of the circular cylinder with the ellipse replacing the cylinder. The same 'O' grid blocking strategy was used to mesh the domain.

The flow past a circular cylinder and an ellipse have similar physics with the ellipse having less separated flow at $0^{\circ}$ angle of attack. Hence the mesh parameters used for this simulation were the same as that of the cylinder. The $k-\omega$ SST turbulence model and the same discretization schemes as that of the cylinder simulation were used.

The aspect ratio (AR) of an ellipse, ratio of major to minor axis, is another influencing factor on the drag coefficient. A higher aspect ratio ellipse will have a lower drag coefficient, and the drag coefficient decreases with increasing aspect ratio until it asymptotes to the value of drag coefficient for a flat plate with the least drag coefficient. But a change in the angle of attack will have a larger effect on the lift force on a higher aspect ratio ellipse than a lower aspect ratio ellipse as will be discussed in the next section. Hence in choosing 


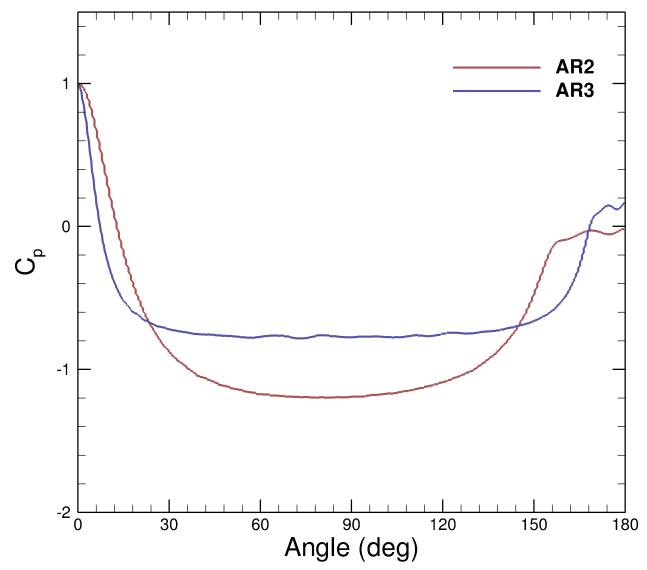

Figure 4.7: Mean pressure coefficient on the surface of ellipses of different aspect ratios.

an elliptical support structure, both these factors will be taken into consideration.

\section{Influence of aspect ratio}

As discussed earlier, the aspect ratio of an ellipse can have a large influence on its force coefficients. To study this influence, two different ellipses of the same frontal area with aspect ratios of 2 and 3 were analysed.

Table 4.3: Influence of Aspect Ratio on Ellipse

\begin{tabular}{|c|c|c|}
\hline Aspect ratio & $C_{D \text { Mean }}$ & Separation angle \\
\hline $2: 1$ & 0.085 & $154^{\circ}$ \\
\hline $3: 1$ & 0.04 & $168^{\circ}$ \\
\hline
\end{tabular}

The ellipse with AR 3 has a lower drag coefficient than AR 2 due to a delayed separation point and a smaller pressure difference; see table 4.3. Note that both these drag coefficients are considerably lower $(\sim 70 \%)$ than that of a circular cylinder. The comparison with circular cylinder is discussed in detail at the end of this section.

The AR3 ellipse has a lower pressure difference due to its more streamlined shape; see figure 4.7. Note that since this is a $0^{\circ}$ angle of attack case, the mean lift is still zero. The 
influence of angle of attack is analysed in the next section.

\section{Influence of Angle of attack}

An ellipse lacks the perfect symmetry of a circular cylinder and hence the angle of attack can have a significant influence on the transverse lift force on the ellipse, especially at high aspect ratios. This can be a major determining factor as the tidal flows at certain locations have a yaw angle of up to $20^{\circ}$ and also the swirling flow from the turbine rotor can cause a change in the angle of attack on the support structure.

As the angle of attack changes from $0^{\circ}$, the pressure distribution is not symmetric any more on the upper and lower surface of the ellipse; see figure 4.8. This asymmetry in the pressure distribution causes a mean lift on the ellipse. An increase in the angle of attack, causes an increase in the peak suction pressure $\left(C_{p}<0\right)$ increasing the mean lift on the ellipse; see figure 4.8a.

The positive pressure peak $\left(C_{p}>0\right)$ shifts slightly near the front of the ellipse with a change in the angle of attack indicating a change in the stagnation point; see figure 4.8a. Also the point of flow separation on the suction surface (the point where pressure remains constant) moves towards the centre of the ellipse with an increase in the angle of attack.

The peak suction pressure is even higher for the higher aspect ratio ellipse at high angles of attack; see figure $4.8 \mathrm{~b}$. This causes a significant increase in the drag and the mean lift on the higher aspect ratio ellipse which will be discussed in the next section.

\subsubsection{Comparison between circular cylinder and ellipse}

As discussed in the last section, the change in the angle of attack, increases the suction pressure on the ellipse and hence the lift increases. The change in the angle of attack has a larger influence on ellipse of AR 3. The lift on this ellipse is about $80 \%$ higher than the 


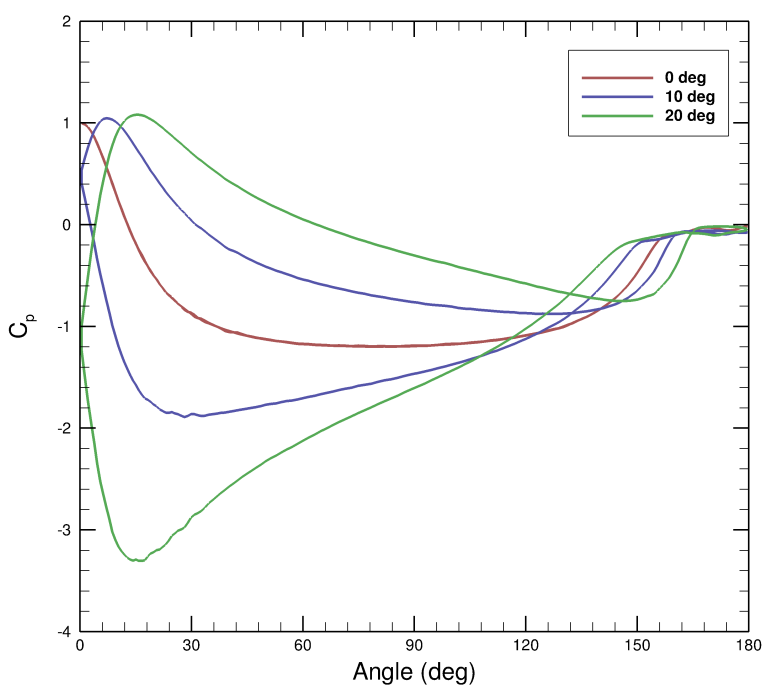

(a) AR 2

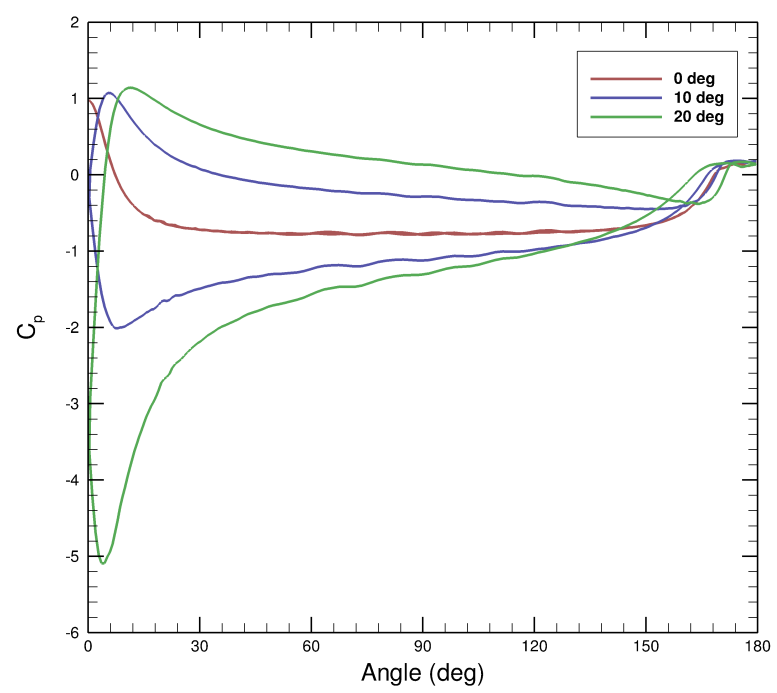

(b) AR 3

Figure 4.8: Mean pressure coefficients at various angle of attack for elliptical cylinders of different elliptic aspect ratios.

ellipse of AR 2 at $10^{\circ}$ angle of attack, and about $70 \%$ higher at $20^{\circ}$ angle of attack; see figure 4.9 .

The change in the angle of attack also influences the drag on the ellipse. At lower angles of attack, $0^{\circ}$ and $10^{\circ}$, the drag on the AR 3 ellipse is lower than the AR 2 ellipse but at higher angle of attack, the drag on the AR 3 ellipse if higher; see figure 4.9. Hence the AR 3 ellipse with a higher drag and a much higher lift at $20^{\circ}$ angle of attack might not be a suitable option for the support structure. Hence the AR 2 ellipse which seems a more plausible option will be analysed.

The mean drag on the circular cylinder is also shown for comparison; see figure 4.9. The drag coefficient of the ellipse is much lower than that of the cylinder. In the case of higher angles of attack, the mean lift force on the cylinder would still be zero but the direction in which lift and drag force act on the cylinder would vary. In this case, the mean drag would have a component in the direction transverse to the flow. Hence it 


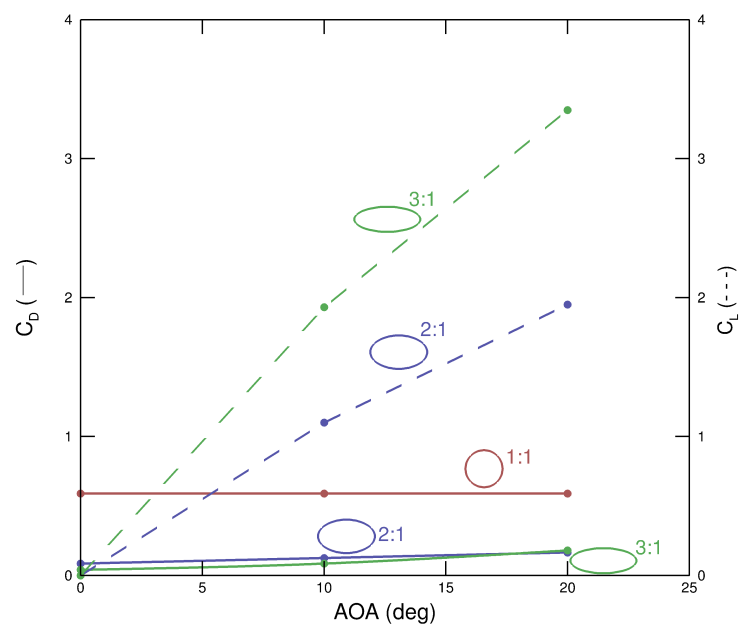

Figure 4.9: Influence of angle of attack (AOA) on the mean drag and lift coefficients of various support structure shapes.

is a combination of both the lift and drag forces on the support structure which would determine the optimum support structure. This will be discussed in Chapter 6 with the support structures in the wake of the turbine rotors.

\subsubsection{Ellipse 3D}

The experimental studies on a 3D ellipse have been considerably fewer compared to those on a cylinder at high Reynolds number. Dikshit (1970) has conducted experiments in the Reynolds number range of $2 \times 10^{4}-10^{5}$ for various aspect ratios of ellipse. In the higher Reynolds number range of $10^{6}$, Delany and Sorensen (1953) performed experiments on an ellipse of aspect ratio 2 and later Heddleson et al. (1957) presented an overview of the drag coefficients on elliptical cylinders of different aspect ratios at Reynolds number of $10^{6}$.

The predicted drag coefficient of the ellipse is slightly lower than the experimental value; see table 4.4. Due to the unavailability of the experimental pressure data on the ellipse, the exact reason for the lower prediction may not be identified. But based on the 
cylinder simulation, where in the pressure recovery was slightly over predicted, it can be inferred that due to the same reason, the drag coefficient is slightly under predicted.

The mean pressure coefficient on an ellipse and a cylinder at mid-section is shown in figure 4.10a. It can be observed that the pressure recovery is higher in the case of elliptical cylinder than in the circular cylinder resulting in a lower drag coefficient.

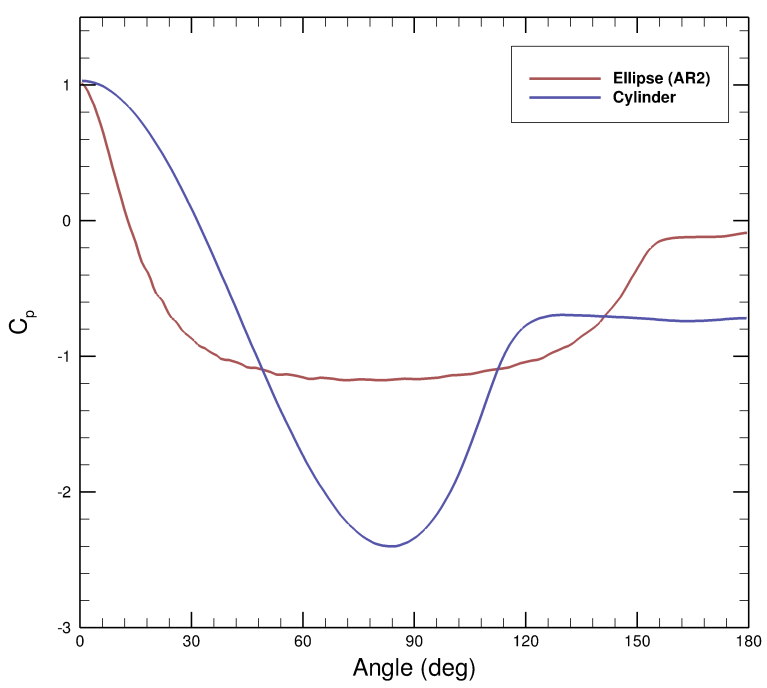

(a) Pressure coefficient

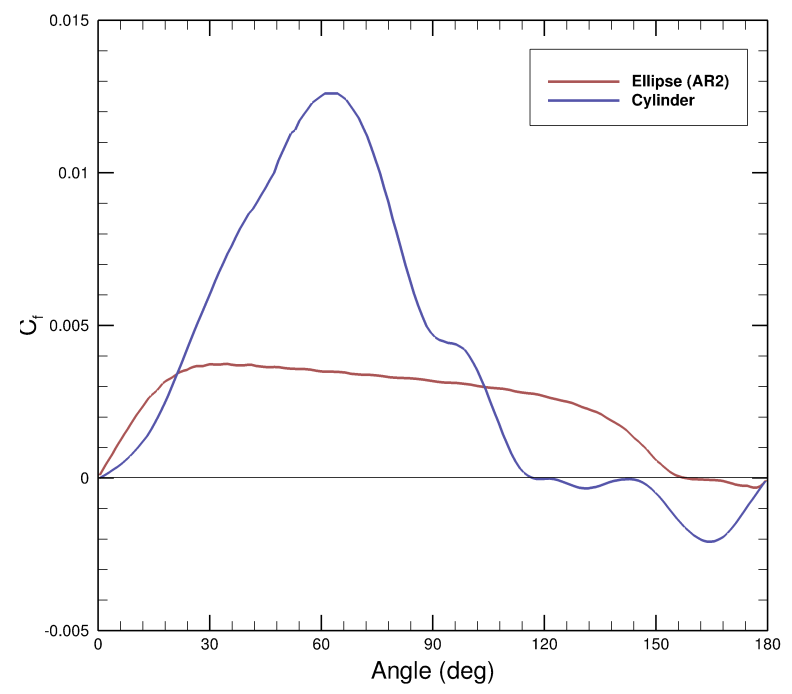

(b) Skin friction coefficient

Figure 4.10: Comparison of Pressure coefficient (a) and Skin friction coefficient (b) between a cylinder and an ellipse.

The pressure coefficient and the skin friction coefficient curves for the ellipse are steeper near the front possibly due to a more rapid change in its curvature than a cylinder; see figure 4.10b. Also the lower peak suction pressure and the lower peak skin friction

Table 4.4: Comparison with experiment

\begin{tabular}{|c|c|c|}
\hline Spanwise elements & $C_{D \text { Mean }}$ & Separation angle \\
\hline Ellipse experimental (Delany and Sorensen, 1953) & 0.16 & - \\
\hline Ellipse (Present work) & 0.15 & $156^{\circ}$ \\
\hline Cylinder (Present work) & 0.59 & $117^{\circ}$ \\
\hline
\end{tabular}


coefficient of the ellipse compared to the cylinder might be due to the smaller change in the curvature of the ellipse, halfway along the streamwise length. For the same reason, the separation point on the ellipse is much later than that of the cylinder.

\subsection{Model scale rotor}

The experimental data available for tidal turbines are limited; one of the more comprehensive experimental studies, which presents rotor force coefficients for a model turbine, was conducted by Bahaj et al. (2007b). This experimental data will be used in this study to validate the computational setup.

Bahaj et al. (2007a) compared the experimental results of Bahaj et al. (2007b) using the commercial code, GH-Tidal Bladed, and their academic in-house code, SERG-Tidal. Their predictions were in close agreement to the experimental results, especially the thrust coefficient $\left(C_{T}\right)$. The power coefficeint $\left(C_{P}\right)$ was predicted accurately at a tip speed ratio of 6 which is the design tip speed ratio of the turbine, with the highest power coefficient. At higher tip speed ratios, there was a slight over prediction by their in-house code but the pattern of decreasing $C_{P}$ still closely followed the experimental results.

Fan et al. (2010) performed a CFD study of this turbine using the commercial code ANSYS Fluent. Their numerical results were in close agreement to the experimental results but the detailed aspects of their study were not mentioned. McNaughton (2013) performed a detailed CFD analysis of this turbine rotor with excellent prediction of the experimental results for most of the tip speed ratios, except when the blade is in the stall region, which is difficult to predict using CFD. McNaughton (2013) presented wake profiles along with the force coefficient data for different turbulence models and different turbulence intensities.

In the present study, the wake profiles will not be studied due to the steady state MRF 
formulation used, which reduces the computational expense significantly compared to mesh rotation. Hence the main aim of this study is to validate the computational setup and the mesh around the turbine.

This section starts with the study of a $2 \mathrm{D}$ airfoil section and then proceeds to the $3 \mathrm{D}$ rotor simulation. The influence of mesh, inlet turbulence and tip speed ratio on rotor force coefficients is discussed in detail and compared with the experimental results of Bahaj et al. (2007b). Lastly, the section ends with a comparison of the steady and unsteady simulations of the turbine rotor.

\subsubsection{Airfoil 2D}

A turbine rotor blade is composed of a number of airfoil sections and hence prior to simulating the entire turbine rotor, the 2D airfoil sections are analysed to obtain an estimate of the intial mesh parameters around the airfoil.

The airfoil sections used in the experiments of Bahaj et al. (2007b) were from the NACA 63-8xx series, xx denoting the airfoil thickness. The experiments were conducted at a range of tip speed ratios with the chord Reynolds number reaching about $5 \times 10^{5}$ at higher tip speed ratios. Hence the 2D airfoil validation is carried out at this Reynolds number. The airfoil chosen for this study is the NACA 63-821 airfoil which is one of the airfoil sections near at the tip of the rotor used in the experiment (Bahaj et al., 2007b).

\section{Geometry}

In modelling the turbine rotor, the sharp trailing edges of the blade would pose a problem in meshing the geometry. Herrig et al. (1951) has described a method in which the trailing edge of an airfoil can be thickened without significantly affecting its characteristics, by closely maintaining its shape. According to this method, the trailing edge was thickened 


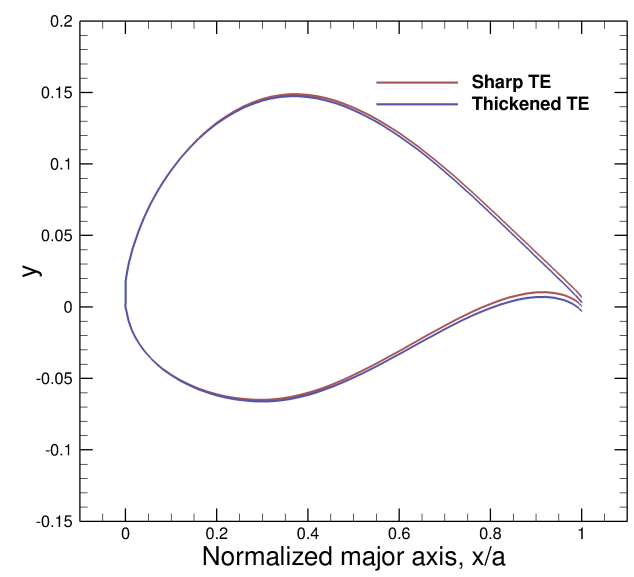

Figure 4.11: Thickened trailing edge (TE) of a NACA 63-821 airfoil

by increasing the thickness linearly along the chord:

$$
\Delta y=(0.0015 x)\left(\frac{t / c}{0.10}\right)
$$

where $t / c$ is the thickness to chord ratio of the airfoil with $x=0$ at the leading edge and $x=1$ at the trailing edge. The NACA 63-821 airfoil used for this analysis was thickened using this method; see figure 4.11. It can be noticed that the profiles are very similar near the leading edge and differ only towards the trailing edge.

\section{Computational setup}

A domain similar to that of the $2 \mathrm{D}$ cylinder was used for the simulation except that the dimensions of the domain were based on the airfoil chord instead of the cylinder diameter.

The inlet and outlet were assigned as a velocity inlet and a pressure outlet respectively. The simulations were carried out for a range of angles of attack, but instead of changing the airfoil orientation at each angle of attack, the boundary conditions were changed. Hence the bottom boundary is assigned as a velocity inlet and the top boundary as a pressure 


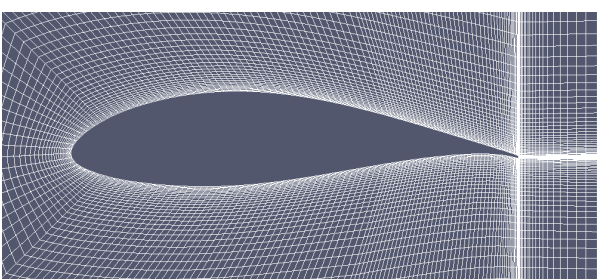

(a) Airfoil

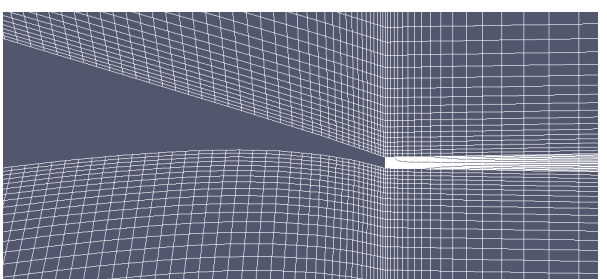

(b) Airfoil Trailing edge

Figure 4.12: Mesh around the airfoil

outlet.

The $k-\omega$ SST turbulence model was used for the simulation. The one-equation Spalart and Allmaras (1992) model is also known to produce good results for an airfoil at a lower computational cost, but the $k-\omega$ SST model was used in order to be consistent with other bluff body simulations performed so far, because all of them will be simulated together in the full-scale turbine model. In the case of the airfoil, the flow separation is expected to be low for most of the angles of attack and hence wall functions were used to reduce the mesh count and hence the computational cost.

The inlet velocity is specified such that the chord based Reynolds number is $5 \times 10^{5}$ as discussed earlier. The freestream turbulence parameters, $k$ and $\omega$ are specified in the same manner as in the previous sections.

\section{Blocking strategy}

A structured C-grid was used for meshing the airfoil; see figure 4.12a. At higher angles of attack, there might be flow separation towards the trailing edge and hence the number of mesh elements are increased here; see figure 4.12b. The thickened trailing edge can also be noticed in figure $4.12 \mathrm{~b}$.

The first cell height on the airfoil is chosen such that the minimum $y^{+}$is around 30 , as the wall function approach is being used. This is to reduce the cell count compared to 


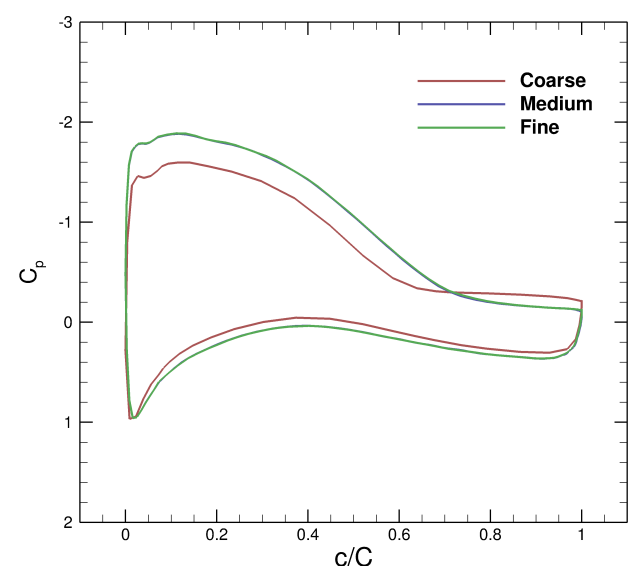

Figure 4.13: Pressure coefficient comparison on different meshes at $8^{\circ}$ angle of attack.

a fully resolved mesh, and the same approach is followed while simulating the $3 \mathrm{D}$ turbine rotor which will be discussed later.

\section{Mesh Independence}

The number of nodes along the airfoil chord and in the direction normal to the airfoil surface can affect the flow physics. The pressure coefficients $\left(C_{p}\right)$ are analysed for different number of mesh elements at $8^{\circ}$ angle of attack; see figure 4.13. This angle of attack was chosen as the lift to drag ratio is optimum at this angle for this airfoil. Note that the negative $C_{p}$ values are plotted on the upper Y-axis to represent the suction surface of the airfoil as per convention.

The coarse mesh predicts a lower pressure near the leading edge which would result in a lower prediction of the lift coefficient. Also the point of flow separation on the suction surface (the point where pressure remains constant) is predicted earlier using the coarse mesh. This might be due to higher diffusion because of the coarser elements, resulting in an earlier prediction of separation point. The results obtained on the medium and fine meshes had a difference of less than $1 \%$ and hence the medium mesh was chosen for the 
study.

\section{Comparison with Experiment}

The airfoil sections in the Bahaj et al. (2007b) rotor experiment belong to NACA 63-8xx series but there are no experimental data available for this series. Instead, a NACA 63-415 airfoil belonging to a similar family was simulated and compared with the available experimental results at the Reynolds number of $7 \times 10^{5}$ which is close to the design Reynolds number.

The experimental data (Abbott and von Doenhoff, 1959) had lift and drag coefficients at different angles of attack but no data about the pressure variation on the airfoil surface. Hence XFOIL (Drela, 1989), a panel based solver, was used to obtain the pressure on the airfoil surface. The results were compared for different angles of attack; see figure 4.14.

At 0 deg angle of attack, a small difference can be noticed between CFD and XFOIL results at $c / C=0.5$; see figure $4.14 \mathrm{a}$. This is possibly due to the transition of the flow from laminar to turbulent which is expected to happen at this Reynolds number. The CFD code assumes the flow to be entirely turbulent and is unable to predict the transition. This might not be a significant concern as in a 3D turbine simulation, the flow is mostly turbulent.

At higher angles of attack, the predicted flow physics starts to deviate between CFD and XFOIL; see figure 4.14b. In the stall region, the flow physics is entirely different with different predictions of peak pressure and flow separation where XFOIL predicts a higher peak and a delayed flow separation; see figure 4.14c. This would affect the lift and drag predictions for the airfoil using XFOIL and CFD.

The lift on the airfoil is in close agreement with the experimental results for lower angles of attack with a maximum error of about $4 \%$ until $8^{\circ}$; see figure $4.15 \mathrm{a}$. At higher 


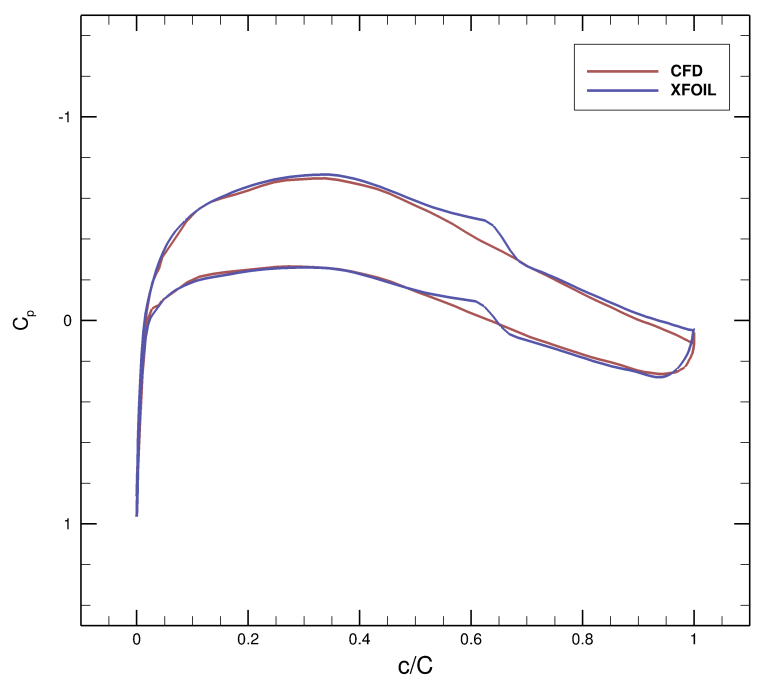

(a) $\mathrm{AOA}=0 \mathrm{deg}$

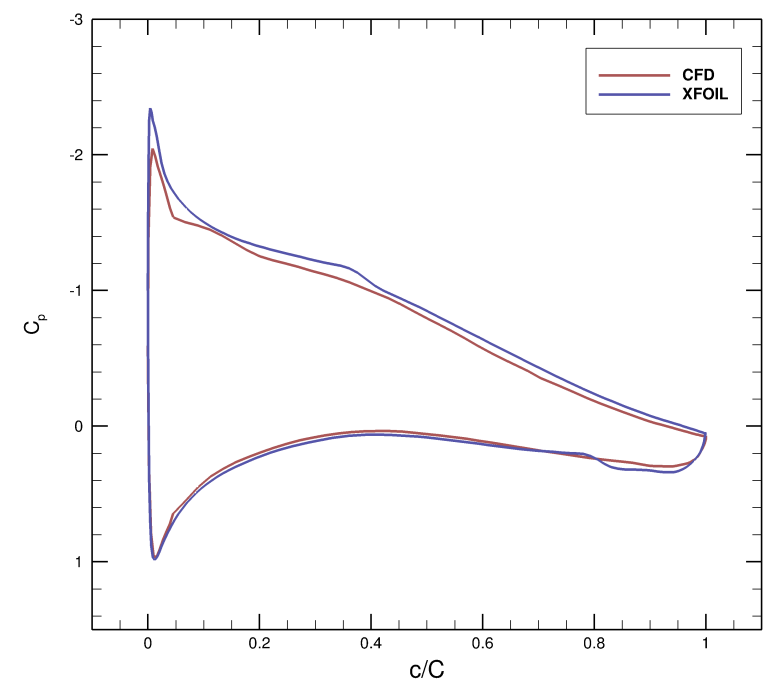

(b) $\mathrm{AOA}=6 \mathrm{deg}$

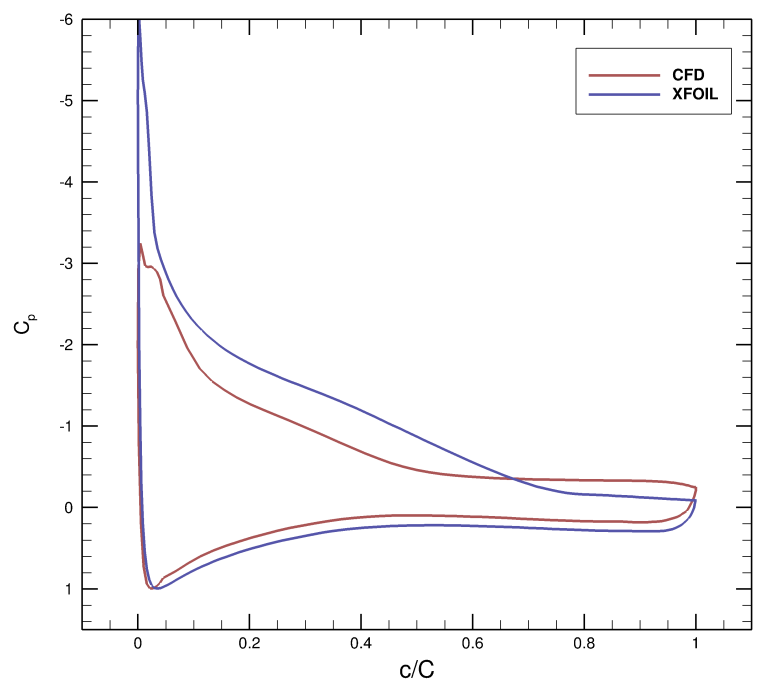

(b) $\mathrm{AOA}=12 \mathrm{deg}$

Figure 4.14: Comparison between pressure coefficients $\left(C_{p}\right)$ predicted by CFD and XFOIL at different angles of attack (AOA). 


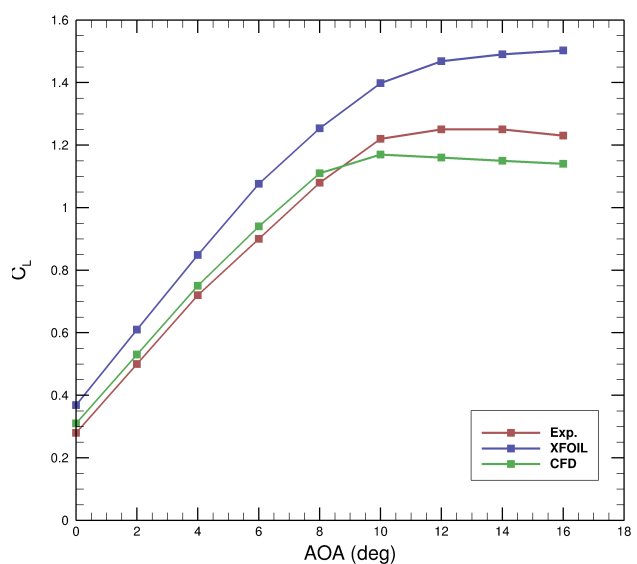

(a) $C_{L}$

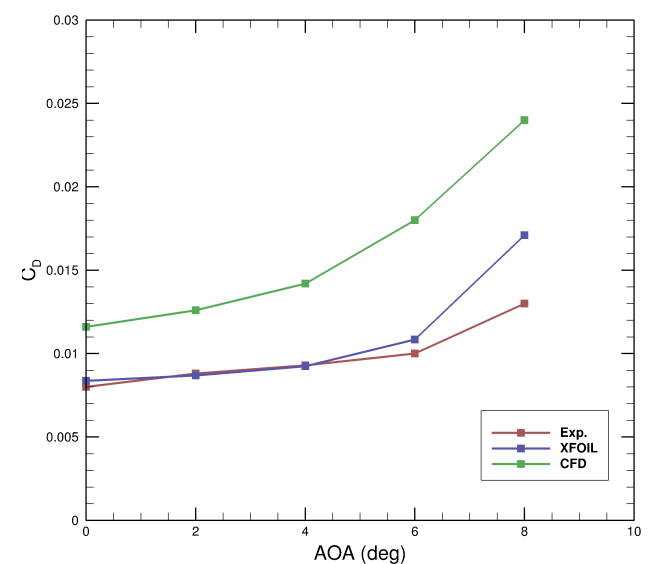

(b) $C_{D}$

Figure 4.15: Lift and drag coefficients of the airfoil for different angles of attack (AOA).

angles of attack, with the onset of stall, it is not possible to achieve an accurate prediction of lift coefficients. The prediction of lift coefficient is better than XFOIL, where XFOIL predicts higher lift due to its higher prediction of pressure on the surface as discussed before; see figure 4.14 .

The drag coefficient on the airfoil is clearly over-predicted using CFD. This is because there is a certain portion of the airfoil near the leading edge where the flow is laminar but the CFD model assumes the flow to be entirely turbulent as discussed earlier. The higher skin friction of a turbulent flow compared to a laminar flow results in the higher drag prediction on the airfoil.

Again the laminar influence on the airfoil might not be of much concern because the flow is mostly turbulent in the simulation of the $3 \mathrm{D}$ turbine rotor as discussed before. Overall the CFD results were in good agreement with the experimental results and this mesh will be used as an initial estimate for the 3D turbine rotor simulations discussed in the next section. 


\subsubsection{Turbine Rotor - Multiple Reference Frame approach}

The flow field in the immediate vicinity of the turbine rotor can be simulated by physically rotating the mesh around the turbine, but this is computationally expensive. Instead, the flow field around the turbine can be numerically modelled by solving the flow equations in a rotating reference frame while those of the far-field flow are solved in the inertial frame. Hence the flow in the domain is solved in multiple reference frames (MRF) in steady-state formulation. MRF can also be run in an unsteady formulation but the computational gain would not be as large. The computational expense of the MRF steady state model is almost an order of magnitude less than the mesh rotation model.

A key shortcoming of the MRF model is that the wake properties of the turbine such as the swirl in the wake cannot be captured in the presence of a support structure, as in an unsteady simulation. Also, if the angle of attack is high enough to produce stall on the turbine blades, an unsteady phenomenon, the MRF steady state model may not capture the physics accurately. Given that the stall phenomenon is difficult to predict even using unsteady CFD formulation, it should not hinder the possible use of MRF to reduce the computational expense drastically.

At angles of attack below stall, the flow is steady except near the root of the blade where the blade joins the hub and has a circular cross-section. However, the negligible influence of blade root on the rotor torque and therefore the power coefficient, means that this can be neglected. Another cause of unsteadiness in the flow arises due to the support structure. In the experimental study of Bahaj et al. (2007b), the support structure was located too far away from the rotor to have substantial effect on it (McNaughton, 2013). The support structure is therefore ignored in the present validation study of Bahaj et al. (2007b) experimental data, making the simulation entirely steady except near the root of the blade. 
This section starts with a description of the geometry and mesh generation. The influence of changing the number of mesh elements and other inlet parameters is then discussed. Subsequently a comparison of CFD results with experimental data is presented.

\subsubsection{Geometry}

The turbine blades are composed of a series of airfoils whose data were obtained from Bahaj et al. (2007b); see Table 4.5. These data were used to plot the airfoil coordinates at different spanwise sections of the blade, which were then used to create the blade and nacelle in the commercial meshing software ICEM-CFD (2010).

The airfoil sections are of NACA 63-8xx series, xx denoting the thickness-to-chord ratio. The thickness is greater at the blade root to support the larger shear force and the bending moment at the root, and decreases towards the tip. The pitch of the airfoil sections also varies along the span of the blade to maximise the lift by optimising the angle of attack at each section; see table 4.5.

The turbine blades used in the experiment have a sharp trailing edge, but this would prevent the construction of a block structured grid. Hence the trailing edge of each airfoil section was thickened, in the same manner as discussed earlier in the 2D airfoil analysis, except for the final section near the root of the blade. At this last section, to make a smooth transition from the circular root to the airfoil, the trailing edge was thickened to four times the usual thickness (McNaughton, 2013).

The particular case chosen for comparison with the experimental data (Bahaj et al., 2007b) was with $20^{\circ}$ hub pitch angle, and hence the entire blade was rotated by $5^{\circ}$ such that the root has a pitch of $20^{\circ}$ and the tip has a pitch of $5^{\circ}$, with the blade twist being $15^{\circ}$. 
Table 4.5: Turbine blade spanwise distribution

\begin{tabular}{|c|c|c|c|c|}
\hline $\mathrm{r} / \mathrm{R}$ & Radius $(\mathrm{mm})$ & $\mathrm{c} / \mathrm{R}$ & Pitch distribution(deg) & $\mathrm{t} / \mathrm{c}(\%)$ \\
\hline 0.2 & 80 & 0.125 & 15 & 24 \\
\hline 0.25 & 100 & 0.1203 & 12.1 & 22.5 \\
\hline 0.3 & 120 & 0.1156 & 9.5 & 20.7 \\
\hline 0.35 & 140 & 0.1109 & 7.6 & 19.5 \\
\hline 0.4 & 160 & 0.1063 & 6.1 & 18.7 \\
\hline 0.45 & 180 & 0.1016 & 4.9 & 18.1 \\
\hline 0.5 & 200 & 0.0969 & 3.9 & 17.6 \\
\hline 0.55 & 220 & 0.0922 & 3.1 & 17.1 \\
\hline 0.6 & 240 & 0.0875 & 2.4 & 16.6 \\
\hline 0.65 & 260 & 0.0828 & 1.9 & 16.1 \\
\hline 0.7 & 280 & 0.0781 & 1.5 & 15.6 \\
\hline 0.75 & 300 & 0.0734 & 1.2 & 15.1 \\
\hline 0.8 & 320 & 0.0688 & 0.9 & 14.6 \\
\hline 0.85 & 340 & 0.0641 & 0.6 & 14.1 \\
\hline 0.9 & 360 & 0.0594 & 0.4 & 13.6 \\
\hline 0.95 & 380 & 0.0547 & 0.2 & 13.1 \\
\hline 1 & 400 & 0.05 & 0 & 12.6 \\
\hline
\end{tabular}




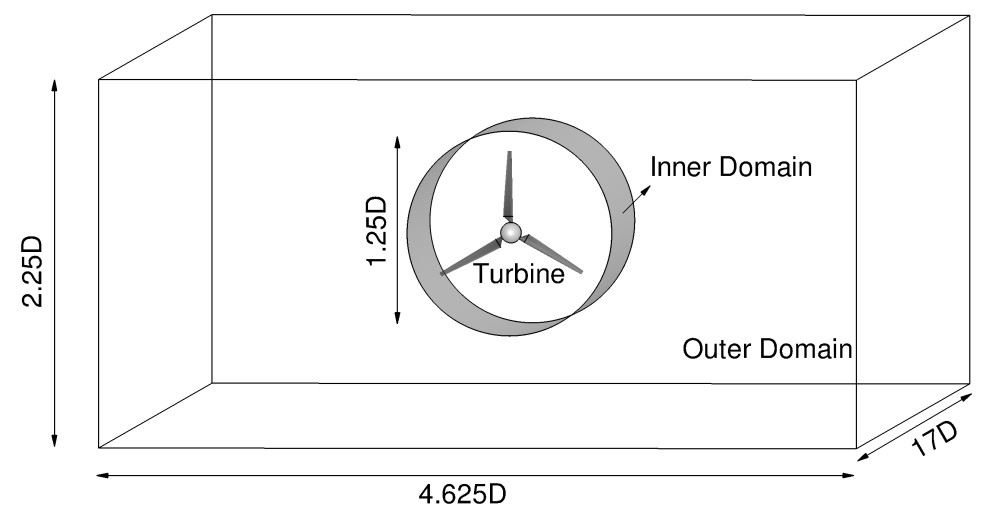

Figure 4.16: Computation domain with inner domain highlighted.

\subsubsection{Computational domain and boundary conditions}

The domain was constructed to achieve the same blockage as that used in the towing tank experiment; see figure 4.16. The inlet and outlet were 5D and 12D respectively from the turbine based on McNaughton (2013). The top and the bottom boundaries were such that the total depth was $2.25 \mathrm{D}$, which was the depth of the towing tank. The turbine was then placed in the domain such that the blade tip immersion depth was $0.55 \mathrm{D}$ (from the top boundary), to simulate the experimental setup. The width of the domain was $4.625 \mathrm{D}$, resulting in a blockage ratio of $7.55 \%$.

To simulate the turbine rotation, either using MRF or mesh motion, a small circular inner domain is constructed close to the turbine, so that the modified equations of MRF or mesh motion are applied only inside this inner domain and the inertial equations are solved in the outer domain. The dimensions of the inner domain need to be large enough to capture the immediate physics behind the rotor but not so large as to increase the computational expense. The circular inner domain has a diameter of 1.25D (McNaughton, 2013). The data transfer between the inner domain and the outer domain is done through the AMI interface as discussed earlier.

The outer domain inlet and outlet are defined as a velocity inlet and a pressure outlet 


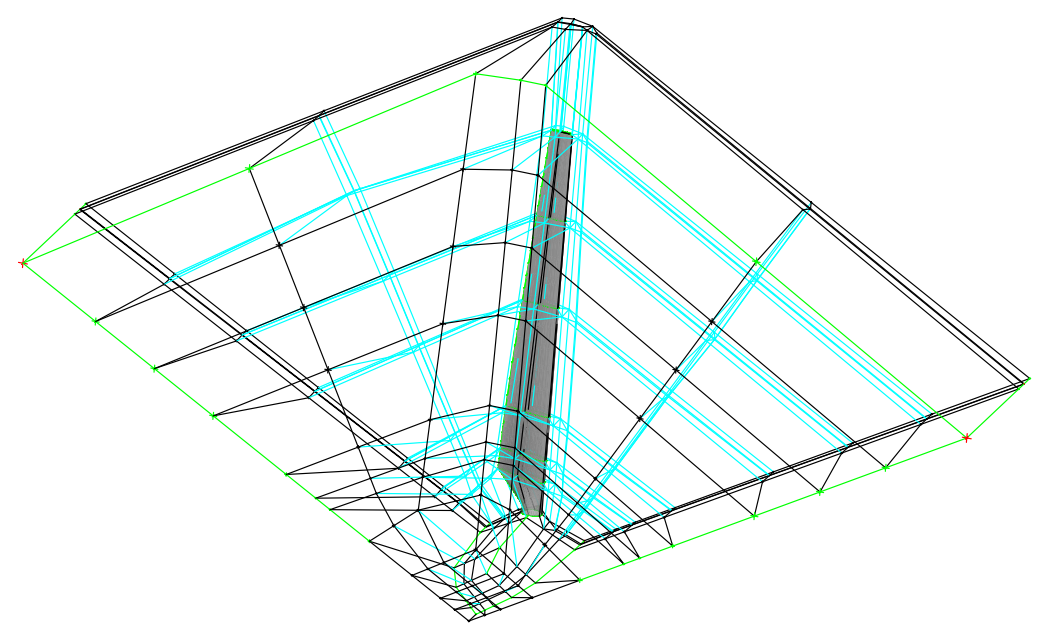

Figure 4.17: Blocking for a $120^{\circ}$ wedge of the Inner Domain.

respectively. In the experiment, the turbine was towed through still water and therefore the side and bottom wall effects are negligible. The free surface of the water is assumed to be fixed. Hence the remaining outer domain boundaries are defined as symmetry planes.

\subsubsection{Blocking strategy}

To create a structured mesh, the domain is first split into blocks such that the mesh is created by following these blocks. The meshing in the inner and outer domains is done separately. This results in a non-conformal interface between these domains where data transfer is carried out using the AMI approach of OpenFOAM (2015).

To mesh the circular inner domain, the symmetry of the rotor is utilised. Instead of meshing the entire circular domain, a $120^{\circ}$ wedge with one blade was used for creating the blocks; see figure 4.17. This blocking was then copied twice to create the entire blocking for the circular domain.

To mesh the blade, the $\mathrm{C}$ grid blocking strategy was used. The thickened trailing edge facilitates another block emerging from it. Also along the spanwise direction, the blade is 


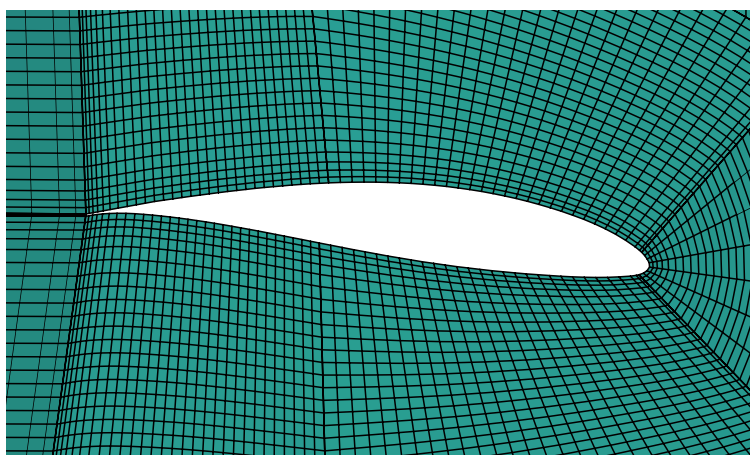

Figure 4.18: C grid around the blade section at $r / R=0.8$.

divided into a number of sections to have better control over the number of elements along the span. This facilitates in creating a fine mesh near the tip of the blade.

On a blade airfoil section, the complex physics associated with flow separation occurs towards the trailing edge of the blade. Hence in the chord direction, the elements were organised such that the mesh is finer towards the trailing edge; see figure 4.18.

\subsubsection{Inlet parameters}

To compare with the experimental results of Bahaj et al. (2007b), the inlet conditions were chosen to closely match the experimental data. The data comparison was undertaken for the $20^{\circ}$ hub pitch angle experiment at tip immersion of $0.55 \mathrm{D}$ with the carriage moving at $1.5 \mathrm{~ms}^{-1}$ in the towing tank. Hence the inlet velocity for the simulation was chosen as $1.5 \mathrm{~ms}^{-1}$.

The freestream turbulence parameters, $k$ and $\omega$, are dependent on the inlet velocity 
$(U)$, turbulence intensity $(I)$ and the turbulent length scale $(l)$ given by

$$
\begin{gathered}
k=\frac{3}{2}(U I)^{2} \\
\epsilon=\frac{C_{\mu}^{\frac{3}{4}} k^{\frac{3}{2}}}{l} \\
\omega=\frac{\epsilon}{k}
\end{gathered}
$$

where $\epsilon$ is the turbulence dissipation rate and $C_{\mu}$ is an empirically calibrated constant in the $k-\omega$ SST model.

The length scale was chosen as 0.7 times the hub height (Brown (2009), McNaughton (2013)). The inlet turbulent intensity should be relatively low as the rotor is moving through still water in the towing tank and hence it was chosen as $5 \%$. The influence of inlet turbulence will be discussed later in the chapter.

\subsubsection{Mesh independence study}

An initial estimate for the number of elements on the circumference of the airfoil section and the growth ratio were obtained from the $2 \mathrm{D}$ analysis of the airfoil section discussed earlier. The first cell height was chosen to maintain the minimum wall $y^{+}$close to 30 given that the wall functions were being used. The mesh count was now varied by changing the

Table 4.6: Mesh Independence study at TSR $=6$

\begin{tabular}{|c|c|c|c|c|}
\hline Mesh & $\begin{array}{l}\text { Inner Domain } \\
\text { mesh count }\left(\times 10^{6}\right)\end{array}$ & $\begin{array}{l}\text { Outer Domain } \\
\text { mesh count }\left(\times 10^{6}\right)\end{array}$ & $C_{P}$ & $C_{T}$ \\
\hline Coarse & 3.1 & 0.41 & 0.425 & 0.761 \\
\hline Medium & 4.5 & 0.41 & 0.445 & 0.772 \\
\hline Fine & 5.6 & 0.41 & 0.447 & 0.773 \\
\hline
\end{tabular}




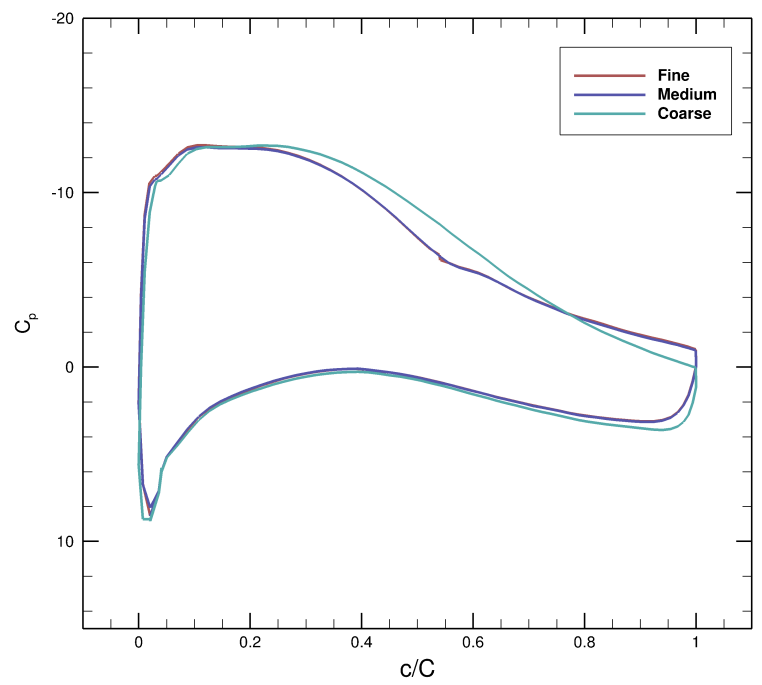

(a) $r / R=0.5$

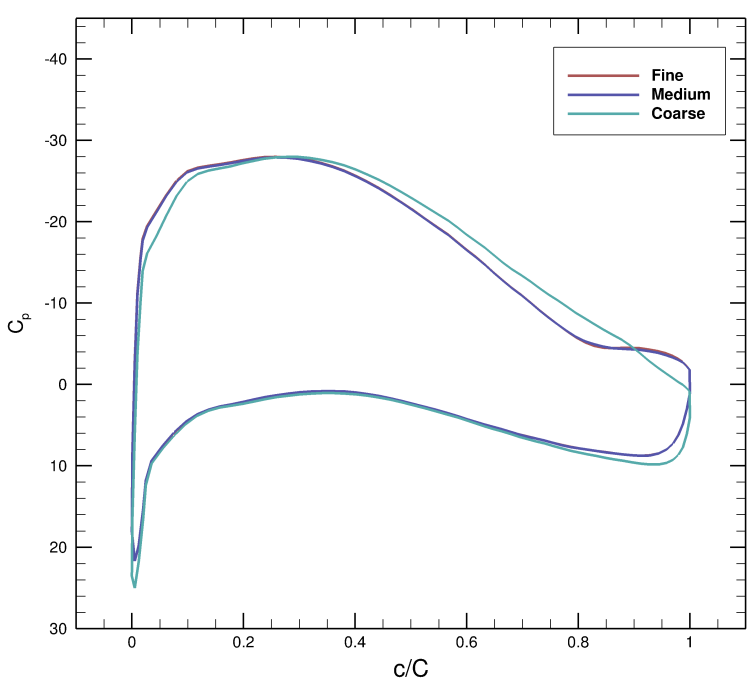

(b) $r / R=0.9$

Figure 4.19: Pressure coefficients at various sections for different mesh count.

number of elements along the span of the blade; see table 4.6. The mesh count in the outer domain was kept constant as it has less influence on the blade force coefficients.

From the experimental data (Bahaj et al., 2007b), the highest power coefficient, $C_{P}$, was at a tip speed ratio, TSR $=6$ and hence the mesh independence study was conducted at this tip speed ratio.

There was a significant difference in the force coefficients between the coarse mesh and medium mesh but the change in the power coefficient between medium and fine meshes was less than $1 \%$, and the change in the thrust coefficient was even smaller; see table 4.6. The pressure coefficients at the mid-section and towards the tip are shown in figure 4.19. Note that the negative Y-axis is upward to indicate the suction surface as per convention.

The pressure prediction on the pressure surface is similar for all the cases but the large difference is on the suction surface. This might be due to the flow separation on the suction surface causing the high pressure gradients which the coarse mesh is unable to resolve. As the mid-section has greater flow separation than at the tip, a larger difference in pressure 
coefficient is noticed at this section; see figure 4.19.

\subsubsection{Inlet turbulence dependency}

The inlet turbulence chosen before was an estimate and since there is no way to determine the inlet turbulence in the experiment, its influence on the results was analysed. Three turbulence intensity (TI) levels are tested in this study, 4\%,10\% and 20\%; see table 4.7. Note that as this is a uniform flow, there is no shear present in the flow to sustain the turbulent intensity. Hence the turbulence intensity close to the blade would be different from the value specified at the inlet.

Table 4.7: Free stream turbulence

\begin{tabular}{|c|c|c|c|}
\hline & TI & $C_{P}$ & $C_{T}$ \\
\hline Low TI & $4 \%$ & 0.442 & 0.767 \\
\hline Medium TI & $10 \%$ & 0.445 & 0.772 \\
\hline High TI & $20 \%$ & 0.497 & 0.851 \\
\hline
\end{tabular}

The change in the force coefficients between low and medium turbulence intensities is minor (about $0.5 \%$ ) but there is a significant difference with the high turbulence intensity case; see table 4.7. The pressure coefficients are plotted at different sections along the span in figure 4.20. The pressure coefficient is very similar for the turbulence intensity levels of $4 \%$ and $10 \%$, while the main difference is with the TI of $20 \%$. Again the larger difference is on the suction surface where there is flow separation.

To study flow separation, the skin friction coefficient at different sections is plotted in figure 4.21. The flow separation point, $C_{f}=0$, is very similar for the low and medium turbulence intensity levels while in the case of higher turbulence intensity, the flow separation is delayed due to the higher momentum flow because to turbulence. This is noticeable 


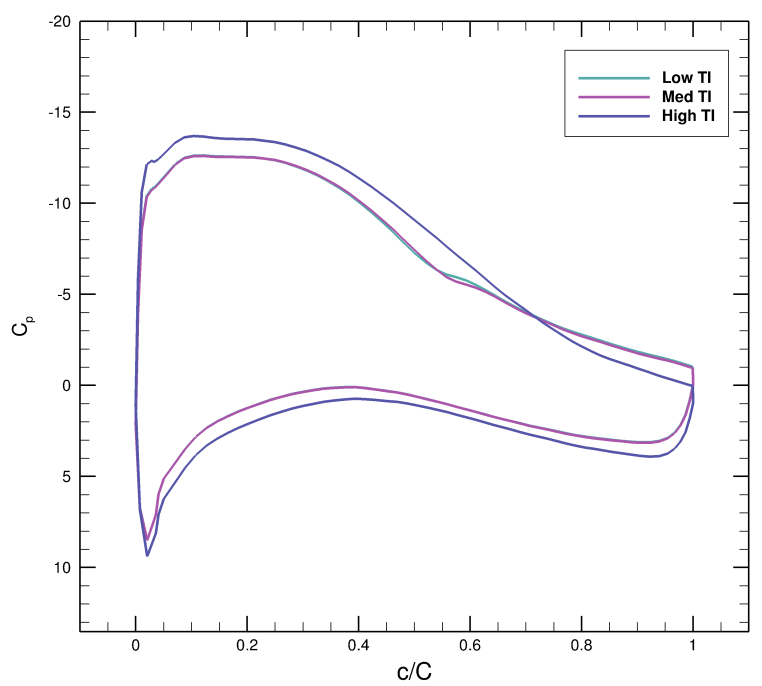

(a) $r / R=0.5$

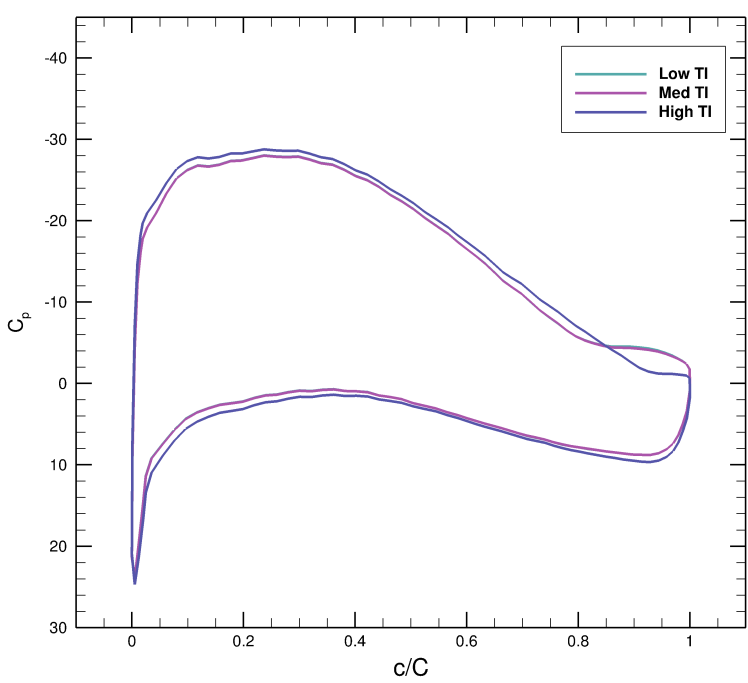

(b) $r / R=0.9$

Figure 4.20: Pressure coefficients at different sections for various TI.

at section, $r / R=0.5$. At section, $r / R=0.9$, due to less flow separation, the changes in the pressure and skin friction coefficient are not as large.

The sectional contours of normalized turbulent kinetic energy (TKE) at $r / R=0.5$ look similar for low and medium turbulence levels while the delayed separation for the higher turbulence case can be noticed in figure 4.22 .

The change in turbulence intensity not only affects the flow around the blades but also the flow in the wake. A high TI may facilitate rapid mixing of the flow and may reduce the velocity deficit as discussed in McNaughton (2013). The wake study is not considered here as the MRF applies only inside the inner domain and the swirl is not propagated into the outer domain to predict wake accurately.

Overall, the turbulence intensity had a minor effect on the force coefficients at low and medium turbulence levels which was likely the case in the towing tank. As long as the freestream turbulence is not so high as to delay separation and change the physics of the flow around the blade, the results would be consistent. Note that in the simulation of a full 


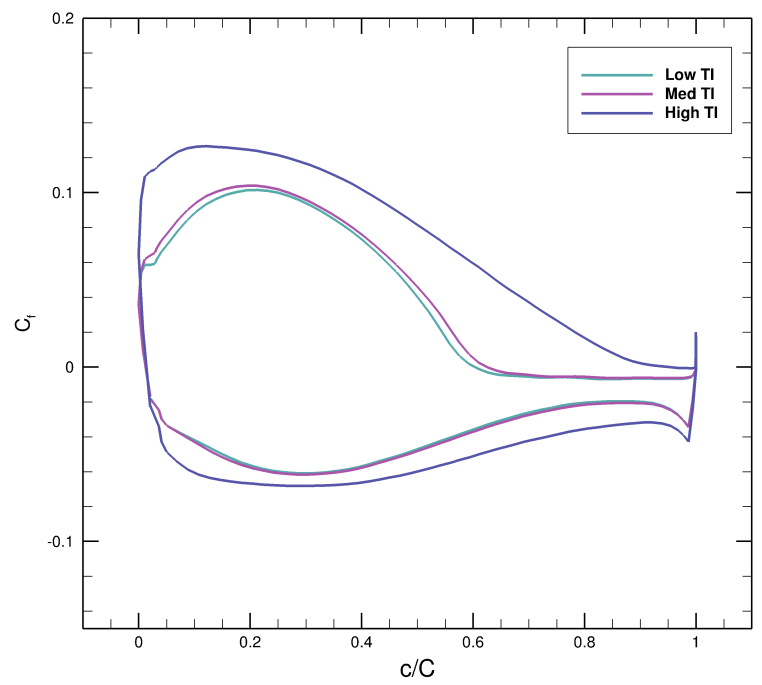

(a) $r / R=0.5$

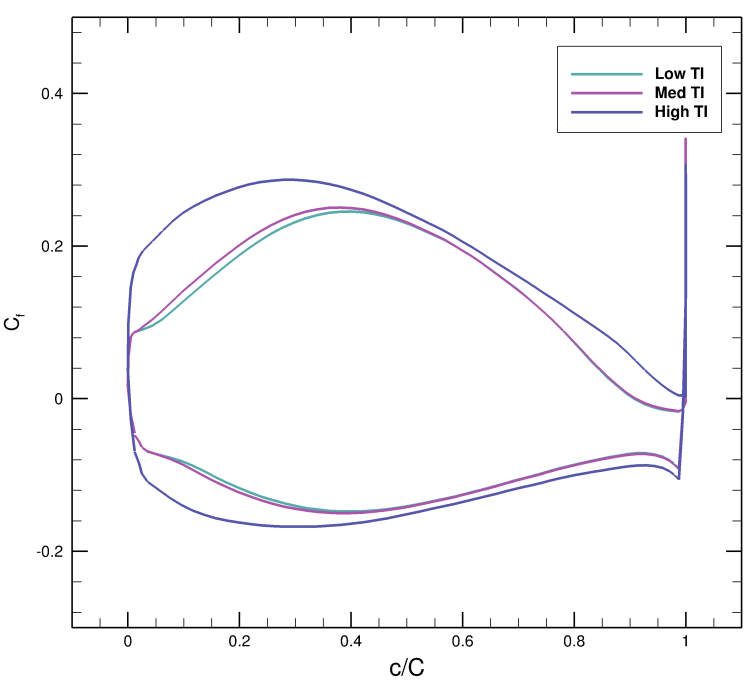

(b) $r / R=0.9$

Figure 4.21: Skin friction coefficients at different sections for various turbulence intensities (TI).

scale turbine, even lower turbulence intensity might play a larger role as it is sustained due to the shear flow in a tidal channel.

\subsubsection{Effect of tip speed ratio}

The experiments of Bahaj et al. (2007b) were performed over a range of tip speed ratios. The change in the tip speed ratio affects the solidity of the rotor, and hence the amount of fluid which passes through it, which in turn affects the power output from the rotor. Also as the tip speed ratio increases, the angle of attack on the blade decreases which affects the pressure distribution on the airfoil sections and hence the turbine force coefficients.

The pressure coefficient around the blade at different sections along the span $(r / R=$ $0.5,0.9)$ for various tip speed ratios is shown in figure 4.23 . The design of the turbine blades is such that a pressure peak at the leading edge of an airfoil section contributes to the torque but as the peak moves away from the leading edge, it contributes more to the thrust 


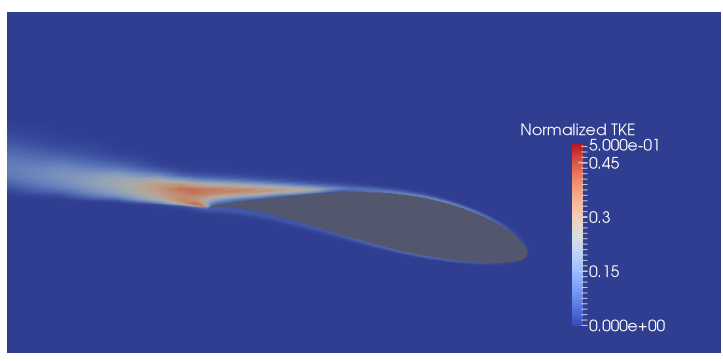

(a) Low TI

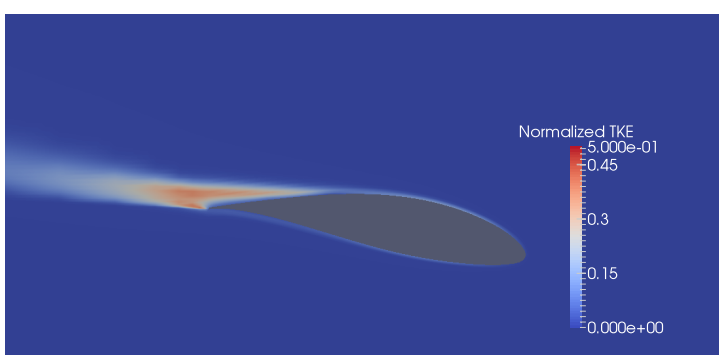

(b) Medium TI

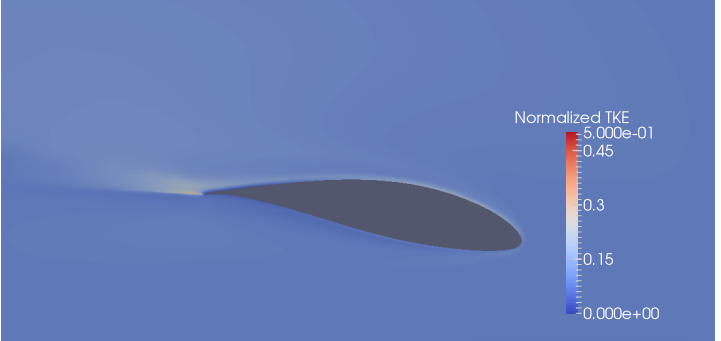

(c) High TI

Figure 4.22: Normalized turbulent kinetic energy TKE, $k / U_{\infty}^{2}$, at $r / R=0.5$ for various turbulence intensities (TI).

on the blade. For TSR 7 and 8, the pressure peak is closer to the centre of the chord which contributes more to the thrust than torque. Also for these tip speed ratios, the pressure on the lower (pressure) surface is negative at certain points which acts as an opposing force to the rotation; see figure 4.23. For these reasons, although the suction peak pressure is higher for higher tip speed ratios, the power coefficient is lower. The force coefficients are discussed in detail in the next section in comparison with experimental results.

The tip speed ratios of 6,7 and 8 follow a similar pressure pattern but the pressure curve for TSR 5 deviates from this behaviour. There is a sharp peak in pressure with a sudden drop compared to the gradual drop for other tip speed ratios. This is more prominent at the mid-section, figure 4.23a, than at the tip, figure 4.23b, due to the higher angle of attack at the mid section. This high angle of attack causes stall and the sudden drop in pressure is responsible for lower torque.

To investigate the stall phenomenon, it is necessary to observe the point of flow separa- 


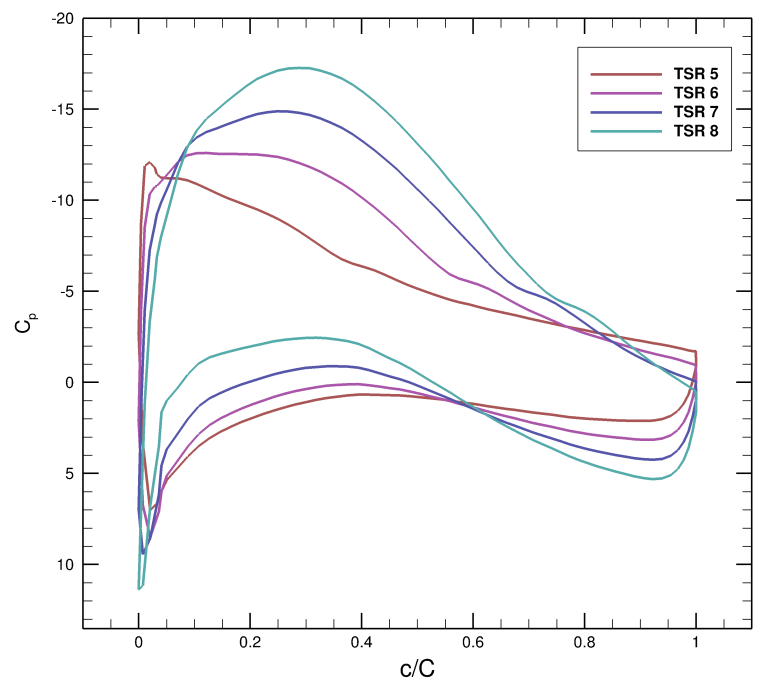

(a) $r / R=0.5$

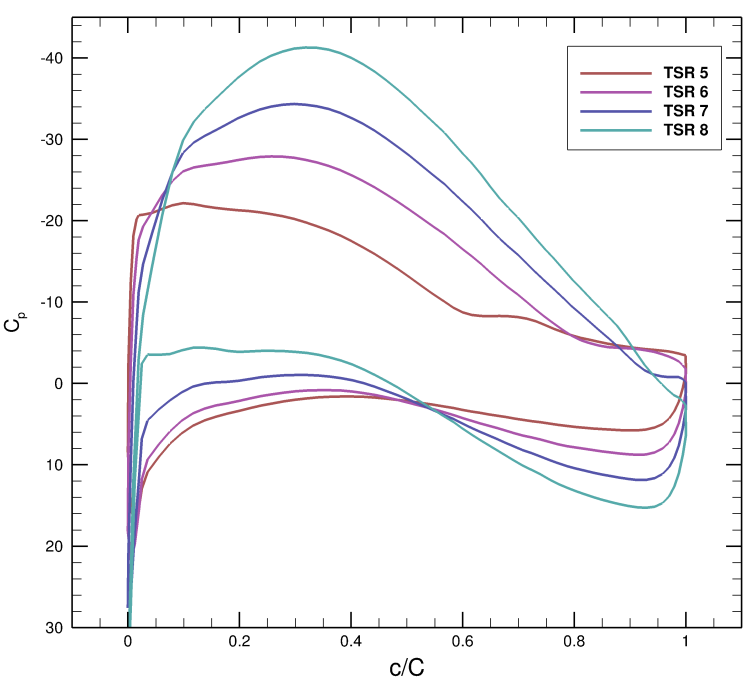

(b) $r / R=0.9$

Figure 4.23: Pressure coefficients at different sections along the span for various tip speed ratios (TSR).

tion, and the skin friction coefficient is a good indicator of flow separation from a surface. The skin friction coefficients for different blade sections over the range of tip speed ratios are shown in figure 4.24. For TSR 5, the flow separates even before half the chord length at the mid-section, $r / R=0.5$, due to stall. Hence it is hard to predict the force coefficients at this TSR. This can be observed in comparison with the experimental data which will be discussed in the next section.

The flow separation point moves towards the trailing edge with an increase in TSR due to the decrease in angle of attack; see figure 4.24a. The flow separation point moves further towards the trailing edge at the tip, $r / R=0.9$, with hardly any separation at higher TSR. This is due to the change in angle of attack experienced by different sections due to the twist in the blade.

The stall leads to high turbulence behind the blade airfoil section at TSR 5; see figure 4.25a. At higher TSR, there is less flow separation and lower turbulence levels around the 


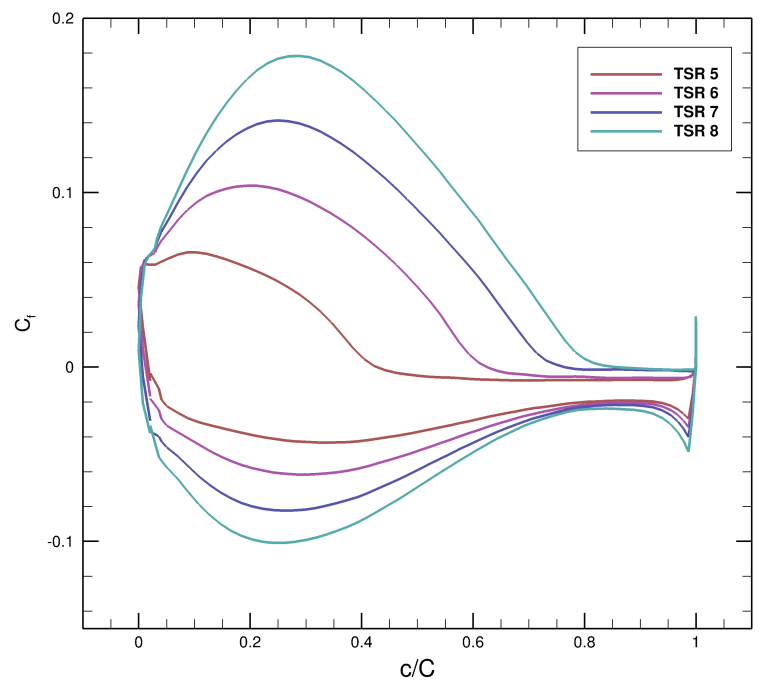

(a) $r / R=0.5$

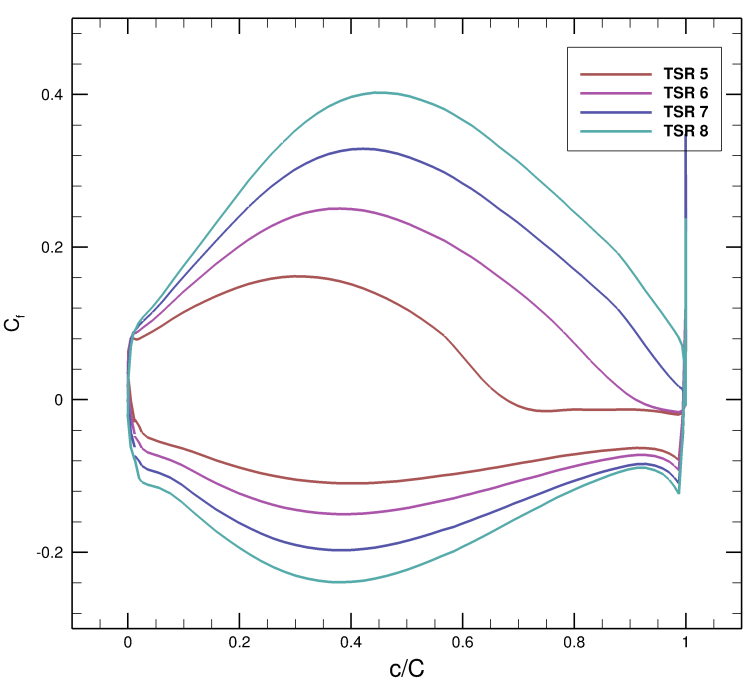

(b) $r / R=0.9$

Figure 4.24: Skin friction coefficients at different sections along the span for various tip speed ratios (TSR).

blade; see figure 4.25 .

\subsubsection{Comparison with experiment}

Bahaj et al. (2007b) present the power and thrust coefficients over the range of tip speed ratios from 5 to 8 . Although the experiments were conducted in a towing tank, the results were presented with blockage correction corresponding to a turbine in open water in an unbounded flow field. The method of blockage correction is briefly discussed below.

\section{Blockage correction}

To compare the CFD results with experiment, the same blockage correction as used in Bahaj et al. (2007b) was applied, which was based on the actuator disc model. The underlying principle of this model is that for the open water conditions, and the given tunnel (blocked) conditions, the flow around the turbine will be similar if the turbine in open wa- 


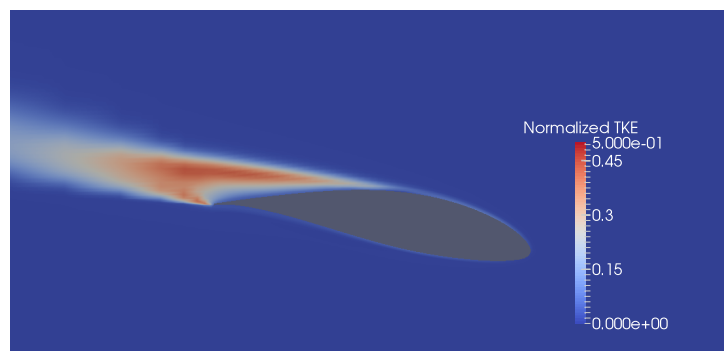

(a) $T S R=5$

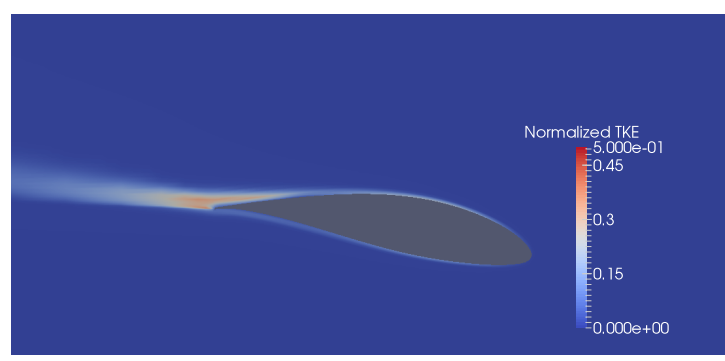

(b) $T S R=7$

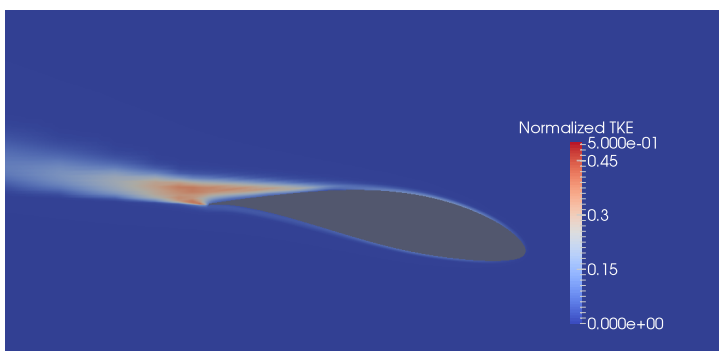

(b) $T S R=6$

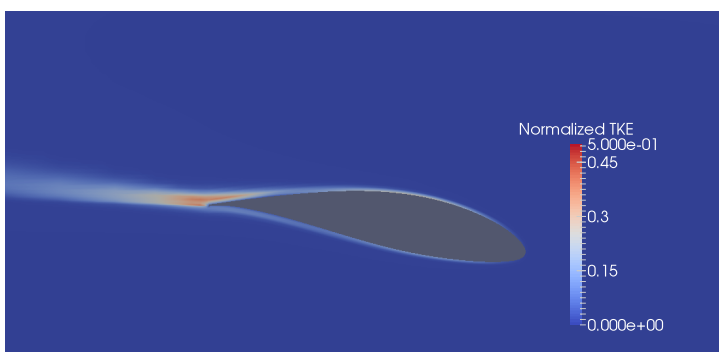

(b) $T S R=8$

Figure 4.25: Normalized turbulent kinetic energy (TKE) at $r / R=0.5$.

ter and tunnel are operating under the same disc flow speed $\left(U_{1}\right)$, turbine RPM and turbine thrust. By comparing these quantities, the blockage correction factor is defined by

$$
\frac{U_{T}}{U_{F}}=\frac{U_{1} / U_{T}}{\left(U_{1} / U_{T}\right)^{2}+C_{T_{T}} / 4}
$$

where $C_{T_{T}}$ is the turbine thrust under tunnel conditions, $U_{T}$ and $U_{F}$ are the inlet velocities in tunnel and open water respectively. Hence, for a given $C_{T_{T}}, U_{1} / U_{T}$ needs to be calculated to obtain $U_{T} / U_{F}$.

In an actuator disc model, the flow is assumed to be uniform across any cross-section of the streamtube enclosing the turbine disc. From Bahaj et al. (2007b), applying the 
continuity and momentum balance equations,

$$
\begin{aligned}
& \frac{U_{T}}{U_{2}}=\frac{U_{3}}{U_{2}}-\frac{A}{C} \frac{U_{1}}{U_{2}}\left[\frac{U_{3}}{U_{2}}-1\right] \\
& \frac{U_{T}}{U_{2}}=1 / \sqrt{\frac{C_{T_{T}}}{\left[\left(U_{3} / U_{2}\right)^{2}-1\right]}}
\end{aligned}
$$

where $A / C$, the ratio of disc area to tunnel area, is the blockage which in the present case is $7.55 \% . U_{2}$ is the wake velocity and $U_{3}$ is the bypass flow velocity. These two equations are solved iteratively to obtain $U_{1} / U_{T}$ and then $U_{T} / U_{F}$ is calculated from equation 4.10.

Based on the blockage correction factor, $\frac{U_{T}}{U_{F}}$, the corrected (open water) coefficients are calculated by

$$
\begin{aligned}
C_{P_{F}} & =C_{P_{T}}\left(\frac{U_{T}}{U_{F}}\right)^{3} \\
C_{T_{F}} & =C_{T_{T}}\left(\frac{U_{T}}{U_{F}}\right)^{2} \\
T S R_{F} & =T S R_{T}\left(\frac{U_{T}}{U_{F}}\right)
\end{aligned}
$$

where the subscripts $\mathrm{F}$ and $\mathrm{T}$ represent open water and tunnel conditions respectively.

The corrected force coefficients are plotted in figure 4.26. Along with the experimental data, the results of McNaughton (2013) are also shown. The predicted values of thrust are close to the experimental results, with an error of about $1 \%$ at the high tip speed ratio and a maximum error of about $6 \%$ at the lowest tip speed ratio (TSR $=5$ ). In comparison to the other CFD study, McNaughton (2013), the results are almost identical with a small difference of about $2 \%$ only at the lowest tip speed ratio. As discussed previously, the lowest tip ratio results in blade stall and hence it is not possible to accurately predict the force in this case. McNaughton (2013) may have achieved a slightly better prediction at this tip speed 


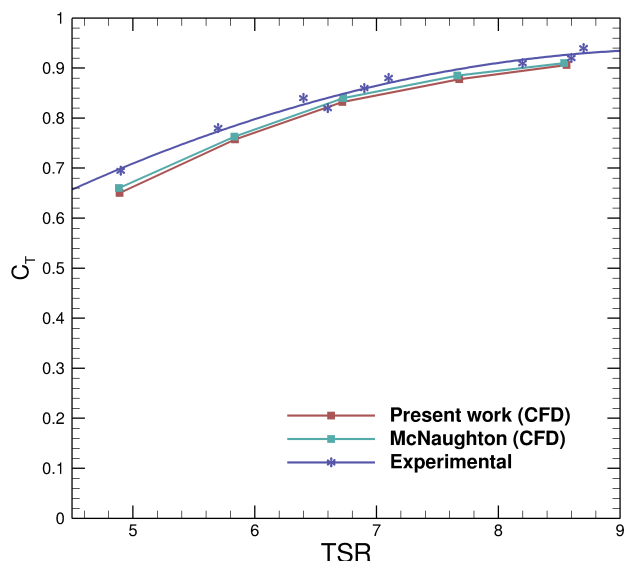

(a) Thrust coefficient, $C_{T}$

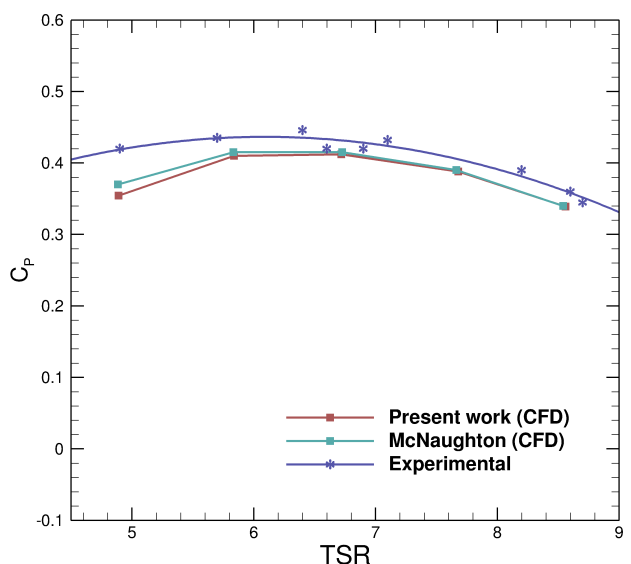

(b) Power coefficient, $C_{P}$

Figure 4.26: Blockage corrected thrust (a) and power (b) coefficients for various tip speed ratios.

ratio due to their unsteady formulation compared to the steady MRF formulation used in the present work. At higher tip speed ratios, the angle of attack on the blade is lower with less separation resulting in more accurate predictions of the experimental results.

As discussed earlier the sudden pressure drop on the suction surface at TSR 5 results in a much lower power coefficient; see figure 4.26b. Also the predicted power coefficient at this tip speed ratio differs from the experimental value by about $8 \%$ but at higher tip speed ratios, this error is reduced to $3 \%$. The error at low TSR is mainly due to the underprediction of lift on the airfoil in the stall region as discussed in the $2 \mathrm{D}$ airfoil analysis. At higher tip speed ratios, better lift prediction at low angles of attack results in accurate estimation of the power coefficient.

At higher tip speed ratios, there is no unsteady separation from the blade except at the root where it joins the nacelle as a circular cross-section. Due to its small lever arm, the blade root does not make a significant difference in the torque and hence power values, which may be the reason for the small difference $(<1 \%)$ in comparison with the results of McNaughton (2013). 


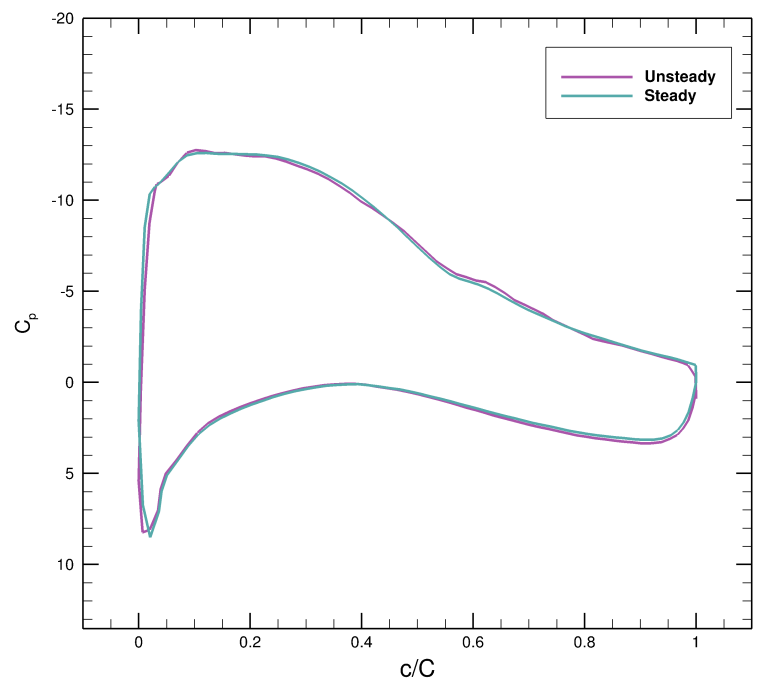

(a) $r / R=0.5$

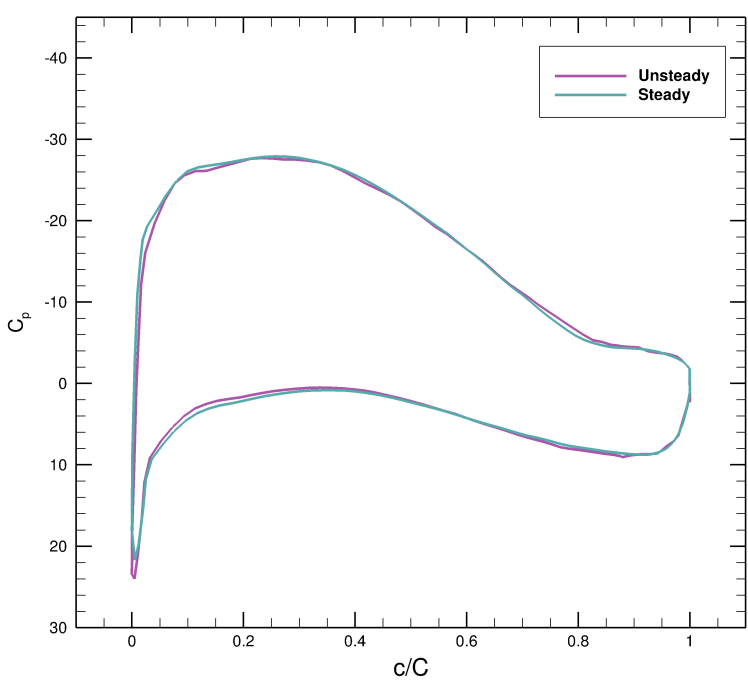

(b) $r / R=0.9$

Figure 4.27: Pressure coefficients at different sections of the blade obtained using different methods of modelling turbine rotation.

\subsubsection{Unsteady mesh rotation comparison}

The analysis so far has been undertaken using the steady MRF formulation. To check the validity of the MRF formulation, an unsteady mesh motion was simulated for one particular tip speed ratio and the results were compared between these two methods.

In this method, the mesh inside the inner domain is physically rotating at the specified angular velocity. This is computationally expensive, but would be essential if the wake effects are to be captured in the presence of a support structure. The present simulation was run for one particular tip speed ratio of 6 which was the design case in the experiments (Bahaj et al., 2007b). Note that the support structure is ignored in these simulations as it was too far away from the rotor and had negligible influence on the turbine force coefficients.

The pressure plots obtained using both the formulations are shown in figure 4.27. To plot the pressure for the unsteady case, the $0^{\circ}$ vertical position of the blade at the top was 
chosen to be consistent with MRF results. The pressure plots are similar at both sections except for a minor difference on the suction surface, where there is flow separation. This only resulted in a change in the force coefficients by about $1 \%$ which is quite small given that the computational expense of the steady MRF formulation is an order of magnitude lower than the unsteady formulation.

At the design tip speed ratio of 6 , there is no unsteady separation from the blade and hence the results from MRF and mesh motion are similar. The only unsteady separation at this TSR might be from the root of the blade where it joins the nacelle as a circular cross-section, but as previously discussed this only has a minor influence on the rotor torque.

At higher tip speed ratios, the angle of attack on the blade is even smaller and the results between MRF and mesh motion are expected to be even closer. This may not be the case at very low TSR.

At TSR of 5, the high angle of attack causes stall and unsteady separation from the blades. At this tip speed ratio, the results would be different from the experimental results as discussed previously. McNaughton (2013) was able to predict the forces better using unsteady formulation but still not close to experimental results; see figure 4.26.

The agreement in force coefficients between the steady and unsteady formulations justifies the use of steady MRF to validate the computational setup.

\subsection{Summary}

Experimental studies on the interaction between a turbine rotor and its support structure were not available to validate the CFD model. Therefore, a building block approach was used in which the support structures and the turbine rotor were separately investigated.

The possible shapes of the support structures, cylinder and ellipse, were analyzed and 
compared with the available experimental data. The prediction of forces and separation angle on the surface were in close agreement with the experimental results.

Among the ellipses of different aspect ratios, AR2 and AR3, the AR2 ellipse had a significantly lower mean lift coefficient overall and a lower drag coefficient at high angles of attack. As a change in the angle of attack on the support structure might ensue due to the yaw in the flow, and also from the swirl in the rotor wake, the AR2 ellipse was chosen for further analysis.

The turbine rotor model was validated using the model-scale experiments performed by Bahaj et al. (2007b). An initial analysis of the 2D airfoil sections was carried out comparing the lift and drag coefficients with the experimental data. The lift coefficients were accurately predicted, but the drag coefficients were over predicted because the turbulence models assume the flow to be entirely turbulent whereas in reality, there is a transition in the boundary layer from laminar to turbulent which was shown by XFOIL. The over prediction of the drag coefficient did not make a large difference in the predicted force coefficients in the 3D rotor simulations as the flow was mostly turbulent due to the turbine rotation.

Later the influence of freestream turbulence on rotor force coefficients was studied. As this was a uniform flow without any shear, the inlet turbulence did not have much influence on the force coefficients except at a very high value of turbulence wherein the flow separation was delayed on the blade.

The effect of tip speed ratio on the force coefficients was then discussed. The force coefficients at various tip speed ratios were analyzed by studying the pressure distribution on the blades. These results were corrected for blockage effects and then compared with the experimental data. The force coefficients were in good agreement with the experimental data except at the tip speed ratio of 5 which was due to the stall phenomenon at low 
TSR.

The steady state MRF formulation was then compared with the unsteady mesh motion for the design tip speed ratio of 6 . The force coefficients and the pressure distribution were in close agreement justifying the use of MRF to validate the computational setup. 


\section{Chapter 5}

\section{Blade pitching in the power-capping}

\section{zone}

\subsection{Introduction}

In the last chapter, the numerical methods were validated with the experimental results of Bahaj et al. (2007b) and were shown to be in good agreement. In this chapter, a fullscale turbine rotor in a tidal channel is simulated using CFD, and the flow and loading characteristics in the power-capping zone due to blade pitching are discussed.

A real tidal channel has a shear profile in velocity instead of a uniform profile. Hence before analyzing blade pitching, the effect of shear velocity profile on the turbine is also studied.

This chapter starts with a description of the full scale rotor and the computational domain used for the simulation, along with the meshing methodology. The influence of shear in the velocity profile on the turbine is then discussed, by comparison to the same turbine in uniform flow. The last section presents the effects of pitching the turbine blades to feather on the rotor force coefficients and on the wake. 


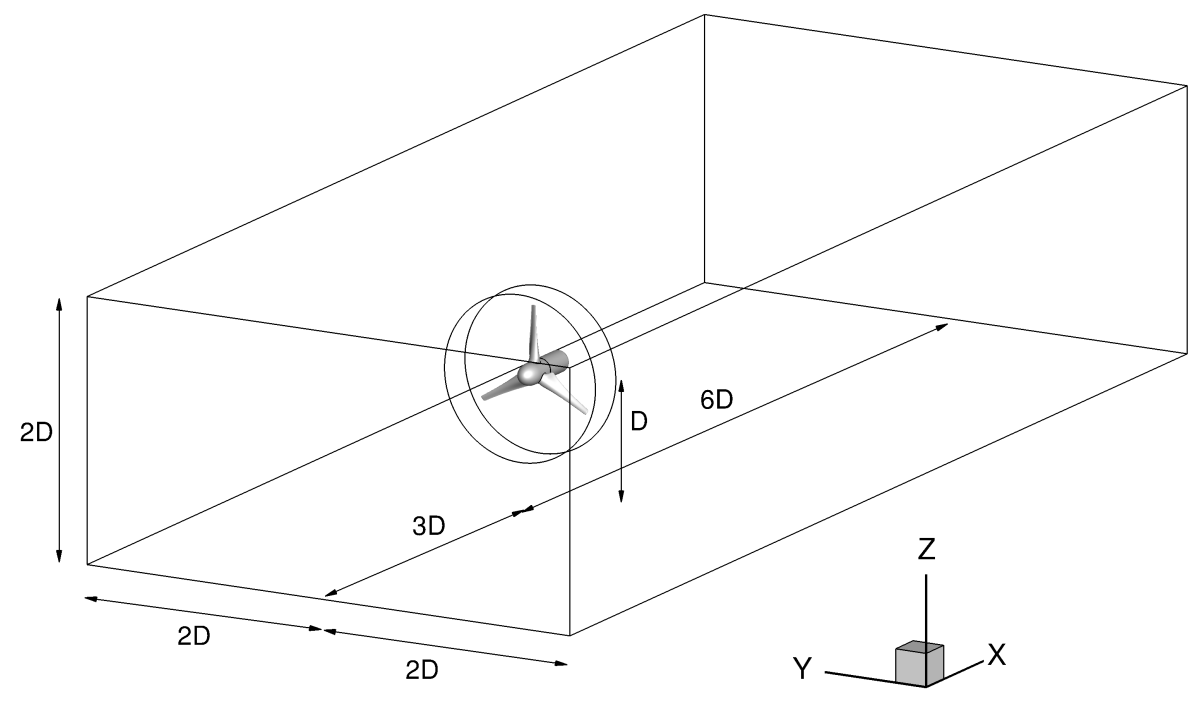

Figure 5.1: Dimensions of the computational domain. The flow direction is from left to right.

\subsection{Turbine rotor description}

\subsubsection{Geometry and domain}

The full-scale turbine rotor is $18 \mathrm{~m}$ in diameter (D). The turbine geometry is based on a proprietary design obtained through the PerAWaT project. The rotor blades are composed of NACA 63-4xx aerofoils, where the section thickness (xx) varies from $18 \%$ at the tip to $55 \%$ at the root. The root of the blades blend into the nacelle in a circular cross-section such that they can be easily pitched. The nacelle has a hemispherical cone at the front and a diameter of $3 \mathrm{~m}$. The rotor is placed in a channel of depth $36 \mathrm{~m}(2 \mathrm{D})$, and width $72 \mathrm{~m}$ (4D), centred both vertically and horizontally; see figure 5.1.

The domain inlet is $3 \mathrm{D}$ upstream of the turbine rotor to allow the flow to stabilise before reaching the turbine and the outlet is $6 \mathrm{D}$ behind the rotor to let the wake stabilise prior to outflow (Fleming, 2014). 
The turbine rotor is placed inside an inner domain in which Multiple Reference Frame (MRF) and mesh motion strategies can be applied as discussed in the previous chapter. This inner cylindrical domain is $0.33 \mathrm{D}$ long and has a diameter of 1.25D (Fleming, 2014). The inner domain size is limited as the cell count inside the inner domain is much higher than that in the outer domain.

\subsubsection{Mesh}

Before simulating the entire 3D rotor, the 2D aerofoils which compose the blade sections were analysed in a similar manner as in the previous chapter in order to optimise the mesh. These mesh parameters were then used in the 3D mesh.

The same blocking strategy as that of the model scale rotor, in section 4.3.5, was used for the full scale rotor. This blocking strategy is employed on a $120^{\circ}$ wedge of the inner domain, and is then replicated twice to mesh the entire inner cylindrical domain. A C-grid was used to mesh the blade with a blunt trailing edge. A number of sections were defined in the block along the blade span to control the spanwise distribution of the mesh. The mesh in the spanwise direction is finer near the tip of the blade than the centre to resolve the three dimensional tip effects of a turbine blade; see figure 5.2a.

The angle of attack on the blade sections along most of the blade span is less than $12^{\circ}$ for the design tip speed ratio. Relatively little flow separation occurs at these angles of attack along the blade except at the inboard sections of the blade, where higher thickness of the aerofoils causes some flow separation. But since the power contribution from the inboard sections is much lower than the rest of the blade, wall functions can be used to reduce the computational cost of resolving the boundary layer on the blade (McNaughton, 2013). Hence the mesh around the aerofoil section is specified such that the wall $y^{+}$is greater than 30; see figure 5.2b. Along the span of the blade, about 50 nodes were used 


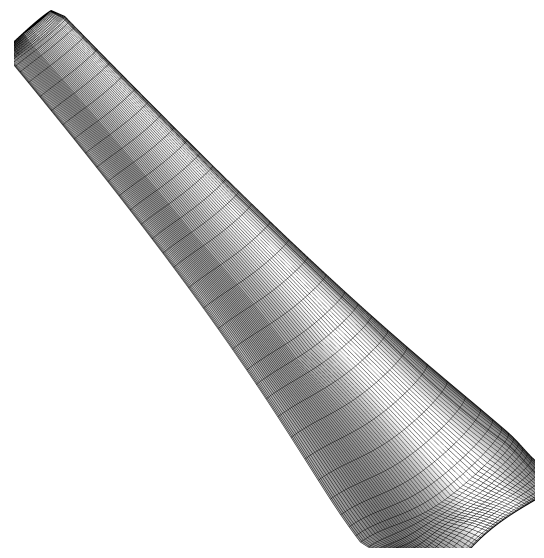

(a) Spanwise mesh distribution on the blade

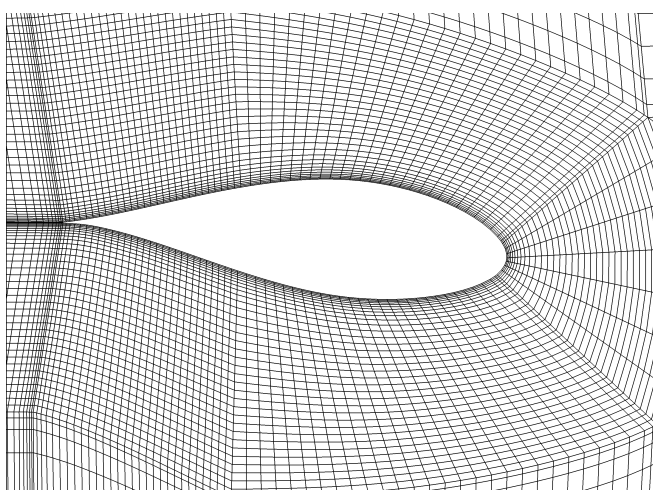

(b) Sectional view of the blade at $\mathrm{r} / \mathrm{R}$ $=0.8$

Figure 5.2: Mesh distribution

for the mesh with finer nodes closer to the tip of the blade; see figure 5.2a. Along the chord of the aerofoil section, about 60 nodes were used on the upper and lower surfaces of the aerofoil with finer nodes towards the trailing edge based on the $2 \mathrm{D}$ validation studies performed. The growth ratio of the cells normal to the boundary was set to 1.1 to capture the boundary effects. The overall mesh count in the inner domain is about 4.77 million and in the outer domain, it is about 1.05 million.

For the unsteady simulations, PimpleDymFOAM solver (OpenFOAM, 2015) was used which uses the PIMPLE algorithm. This removes the constraint of maintaining the Courant number below 1 and hence the time-step was chosen such that maximum Courant number in the domain was below 10. The simulations were carried out for 10 rotor revolutions such that the flow at inlet can reach sufficiently far downstream of the turbine in this time to study the wake properties. 


\subsubsection{Boundary conditions}

\subsubsection{Uniform flow}

The domain inlet and outlet are set as velocity inlet and pressure outlet respectively as discussed in the previous chapter. Both the side boundaries, and top and bottom boundaries are set as symmetry planes.

The freestream turbulence parameters, $k$ and $\omega$, are dependent on the inlet velocity $(U)$, turbulence intensity $(I)$ and the turbulent length scale $(l)$ :

$$
\begin{gathered}
k=\frac{3}{2}(U I)^{2} \\
\epsilon=\frac{C_{\mu}^{\frac{3}{4}} k^{\frac{3}{2}}}{l} \\
\omega=\frac{\epsilon}{k}
\end{gathered}
$$

where $\epsilon$ is the turbulence dissipation rate and $C_{\mu}=0.09$ is an empirically calibrated constant.

The length scale was chosen as 0.7 times the hub height based on the past CFD studies on turbines which were shown to closely match experimental results (Brown (2009), McNaughton (2013)). The inlet turbulent intensity was chosen as $6 \%$ for the uniform flow which is about the depth-averaged turbulence intensity in the shear flow which will be discussed in the next section (Fleming, 2014).

\subsubsection{Shear flow}

In a shear flow, most of the boundaries have the same conditions as that in a uniform flow except the domain inlet and bottom. The inlet boundary conditions are specified based on the shear flow model of Fleming et al. (2013), such that the depth-averaged inlet velocity 


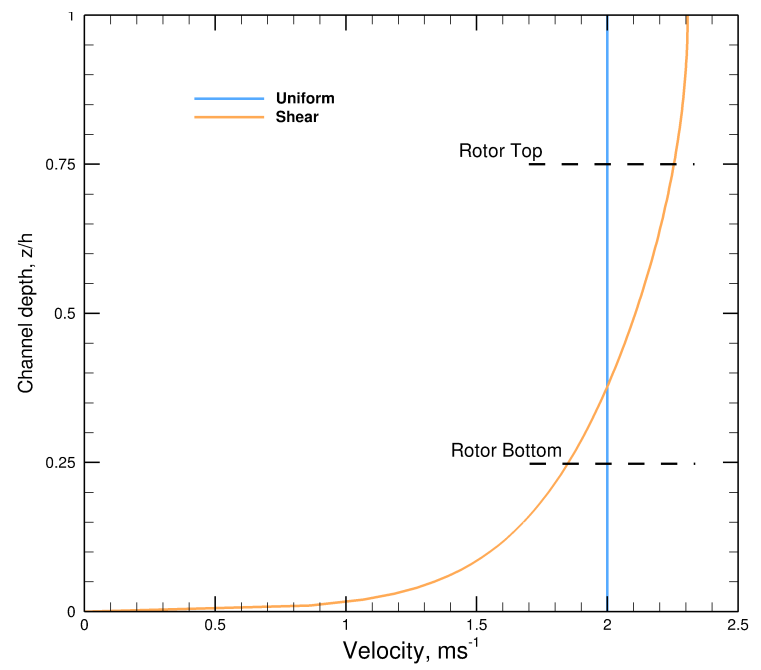

Figure 5.3: Velocity profiles in uniform and sheared flow. Both have a depth-averaged velocity of $2 \mathrm{~ms}^{-1}$. Rotor is located between $0.25 h$ and $0.75 h$ where $h$ is the depth of the channel.

is $2 \mathrm{~ms}^{-1}$ which is the same for the uniform flow; see figure 5.3.

Specifying a constant turbulence intensity at the inlet results in a loss in the shape of the shear velocity profile by the time the flow reaches the turbine (McNaughton, 2013). An alternative method proposed by Fleming et al. (2013) was used wherein the turbulence intensity was varied along the depth of the channel at the inlet to maintain the shear in the velocity profile. Hence the turbulence intensity at the inlet varies from $0 \%$ at the free surface to about $12 \%$ at the channel bottom. As part of their model, a bed shear had to be applied to sustain the shear profile in the flow velocity along the length of the domain which is done by applying a wall shear stress condition to the bottom boundary of the domain. This shear model was implented in OpenFOAM (2015), version 2.3.1, by creating custom libraries. 


\subsection{Flow physics in uniform and shear flow}

In an actual tidal channel, the flow velocity is not uniform through the channel depth but instead has a shear profile. The influence of shear flow profile on a turbine rotor is studied in this section by comparing it with the performance of the same rotor in a uniform flow profile.

The section starts with a discussion of the integrated power and thrust coefficients of the rotor followed by a more detailed analysis of the blade sectional forces along the span of the blade. The flow in the wake is then analysed along with the flow angle of attack along the blade span to investigate the physics governing the rotor behaviour.

\subsubsection{Power and thrust coefficients of the rotor}

The turbine rotor is simulated in uniform flow and shear flow at three different tip speed ratios, $\lambda$, and the time-averaged integrated thrust and power coefficients are presented in figure 5.4. A peak in the power coefficient can be identified at $\lambda=5.5$, which is the design tip speed ratio of the rotor; see figure 5.4a. At this tip speed ratio, the angle of attack on the turbines' blade aerofoil sections is in the optimum range of lift to drag ratio resulting in optimum power. The angle of attack is discussed further in the next section.

As the tip speed ratio increases, the turbine presents more resistance to the flow, thus increasing the thrust on the turbine. This is observed in figure $5.4 \mathrm{~b}$ with the thrust coefficient increasing with tip speed ratio. The higher thrust corresponds to increased axial blade forces and a lower velocity wake behind the rotor.

As the power and thrust on a turbine rotor vary with flow velocity, $U$, as $\mathrm{U}^{3}$ and $\mathrm{U}^{2}$ respectively, the power and thrust are higher for the shear flow case as compared to the uniform flow case due to the higher flow velocities in the rotor swept area in the shear flow; see figure 5.3. 


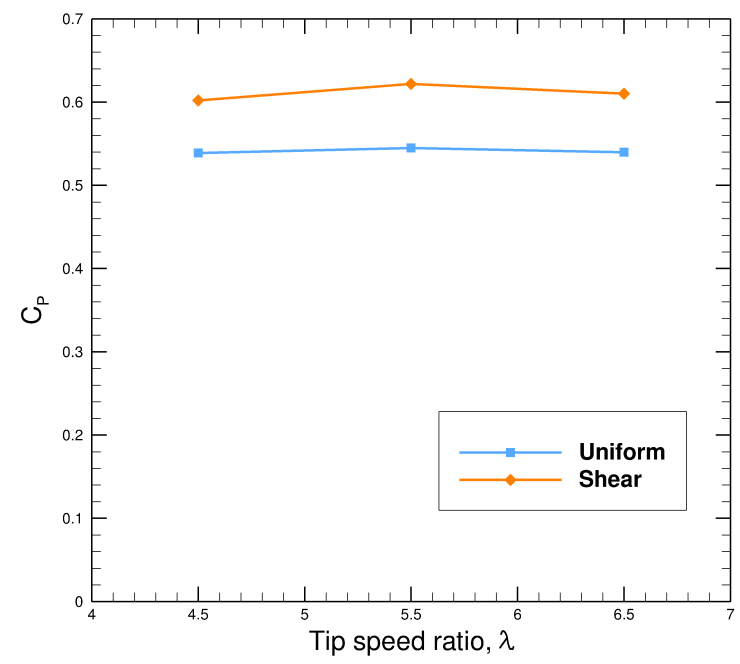

(a) Power coefficient, $\mathrm{C}_{P}$

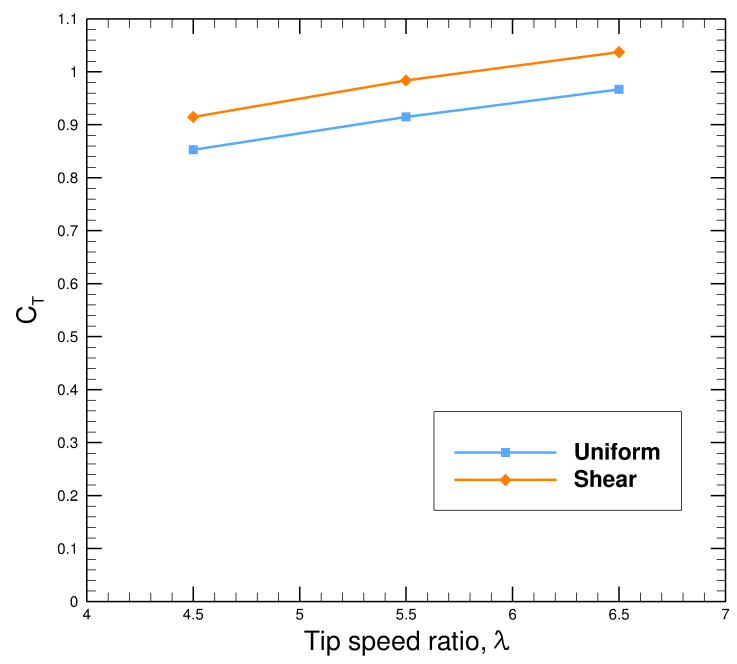

(b) Thrust coefficient, $\mathrm{C}_{T}$

Figure 5.4: Power and thrust coefficients in uniform and shear flow at different tip speed ratios. The depth averaged velocity of $2 \mathrm{~ms}^{-1}$ is used for calculating the coefficients in both uniform and shear flow cases.

In the shear flow case, a turbine blade rotates from a high velocity region to a low velocity region, which results in a change in the blade force coefficients during the rotation cycle. The values of blade force coefficients normalised by the time averaged rotor force coefficients for the design tip speed ratio of 5.5 are presented in figure 5.5. In the uniform flow case, the blade force coefficients are approximately constant as the blade rotates, with the maximum fluctuation being about $1 \%$ possibly due to the anisotropy of the rectangular domain.

The normalised blade force coefficients in shear flow have a much higher deviation about their mean value. The blade power coefficient shows greater sensitivity than the blade thrust coefficient. The maximum power coefficient variation about its mean value is $12 \%$ whereas that of the maximum thrust coefficient variation is about $4 \%$; see figure 5.5. The power coefficient variation is higher due to its $\mathrm{U}^{3}$ variation with flow velocity compared to the $\mathrm{U}^{2}$ variation of thrust. It is important to note that the minimum values 


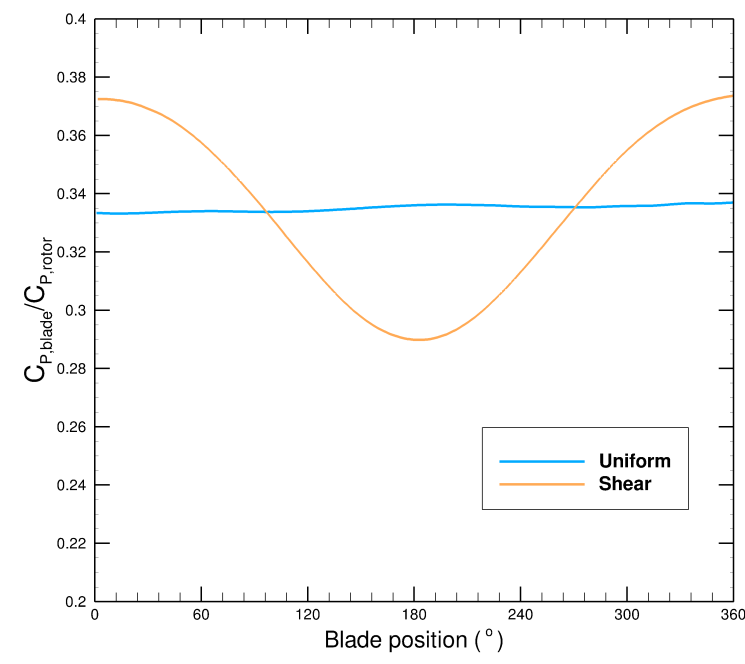

(a) Normalised blade power coefficient

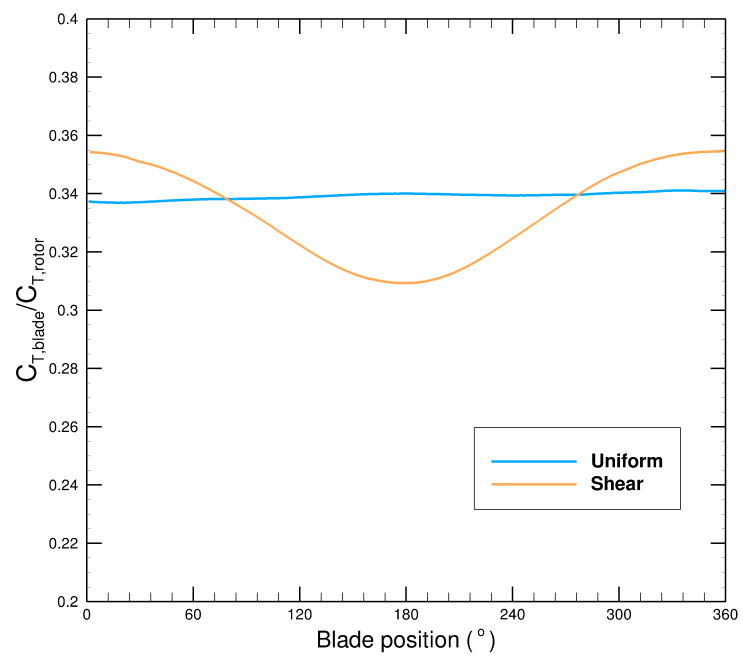

(b) Normalised blade thrust coefficient

Figure 5.5: Blade force coefficients normalised by rotor force coefficients for uniform and shear flow over one turbine rotation; $0^{\circ}$ represents the top most point and $180^{\circ}$ represents the bottom most point.

of the power and thrust coefficients occur at $180^{\circ}$ because the support structure is not included. In the presence of a support structure, the minimum is expected to occur slightly before $180^{\circ}$ due to the rotor interaction with the support structure (McNaughton, 2013) and will be discussed in the next chapter.

\subsubsection{Blade forces}

In the last section, the overall forces on a blade during its rotation cycle were analysed. In this section, the forces on a blade are studied in more detail by analysing the sectional force and angle of attack, $\alpha$, variation along the span of the blade during a rotation cycle.

The force on the aerofoil blade section depends on the approach angle of the flow, $\phi$, and the angle of attack, $\alpha$, on the blade sections which are presented in figures 5.6 and 5.7 respectively. The approach angle is derived based on the method outlined by Wimshurst and Willden (2016). According to this method, the angle of approach is obtained based 


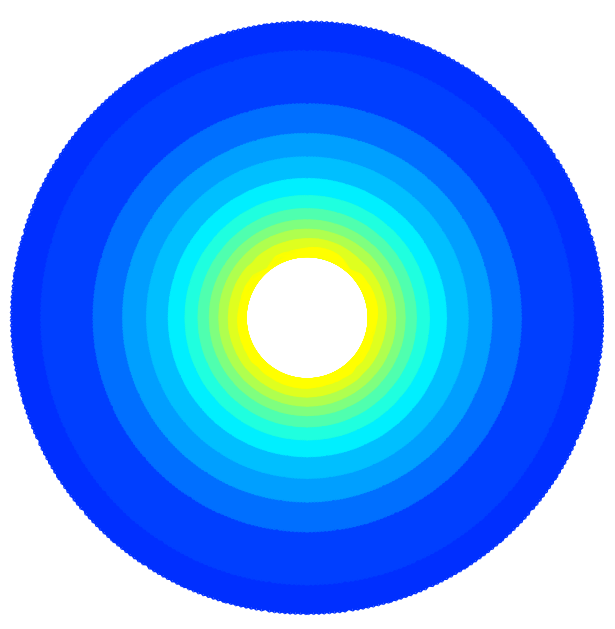

(a) Uniform Flow.

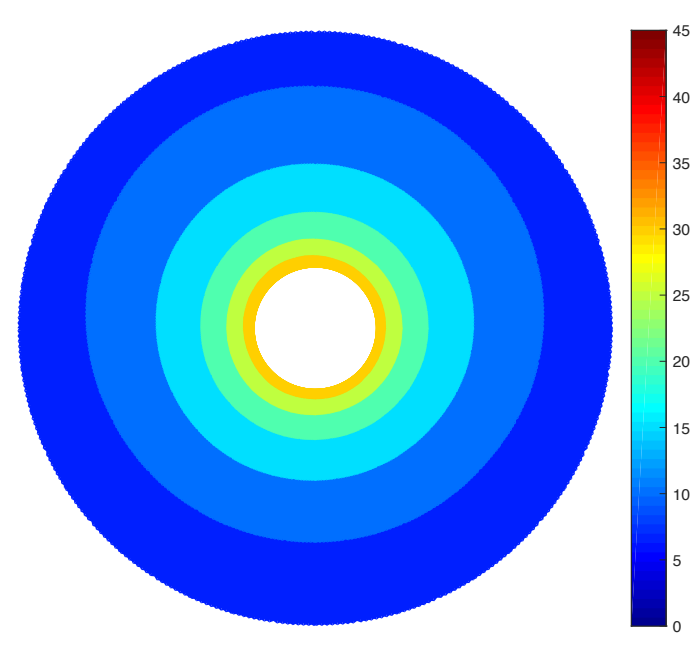

(b) Shear flow

Figure 5.6: Spanwise variation of approach angle, $\phi^{\circ}$, along the blade during a rotation cycle in uniform and shear flow. The depth averaged flow velocity is $2 \mathrm{~ms}^{-1}$ in both cases. Note the vertical asymmetry in the shear flow case.

on the axial flow velocity through the rotor plane and the azimuthal velocity at different points along the span of the rotor blade. The axial flow velocity at the turbine rotor plane is obtained by averaging the axial flow velocity slightly upstream and downstream of the rotor. The azimuthal rotor velocity obtained based on the rotor angular velocity is then used to determine the angle of approach onto the blades. The angle of attack is then attained by subtracting the twist of the blade along its span from the approach angle.

As expected the approach angle variation is azimuthally symmetric in the uniform flow case during one blade rotation whereas in the shear flow case, the approach angles are higher in the top half of the rotation due to the higher axial flow velocity in the shear flow case compared to the uniform flow case; see figure 5.6. This results in a higher angle of 


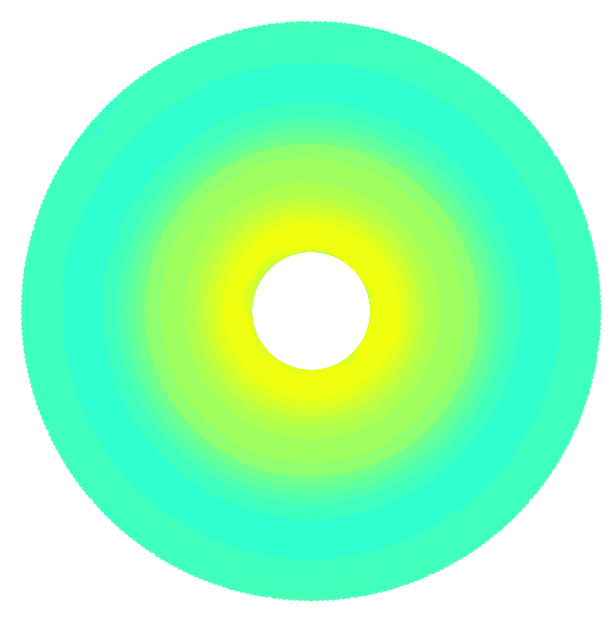

(a) Uniform Flow.

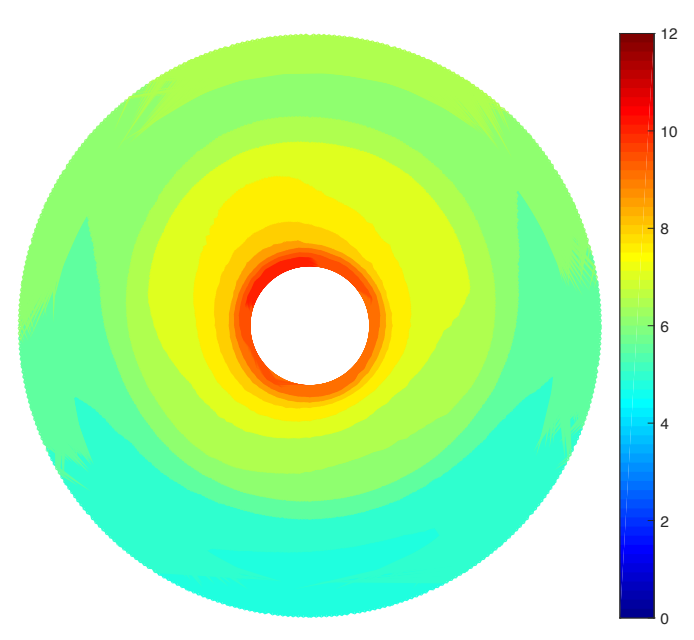

(b) Shear flow

Figure 5.7: Spanwise variation of angle of attack, $\alpha^{\circ}$, along the blade during a rotation cycle in uniform and shear flow. The depth averaged flow velocity is $2 \mathrm{~ms}^{-1}$ in both cases.

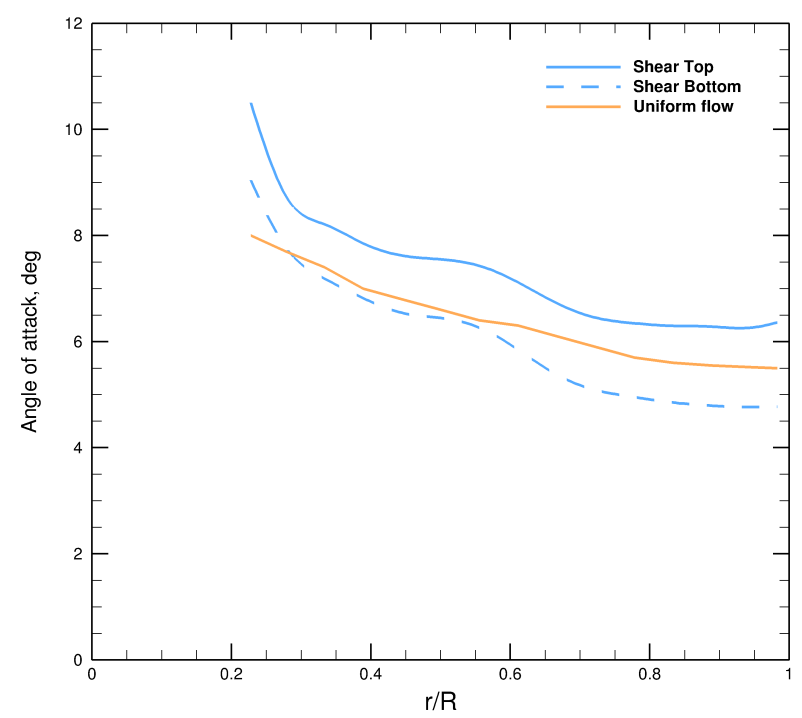

Figure 5.8: Angle of attack variation along the span of a turbine blade for uniform and shear flow at the top (solid) and bottom (dashed) position in a rotation cycle. 


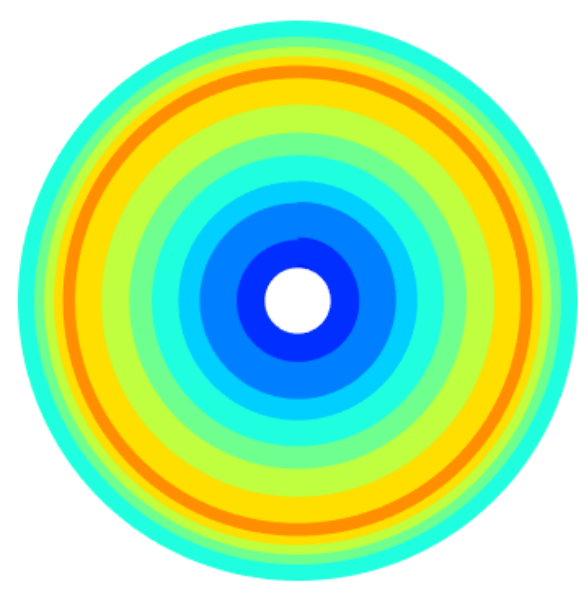

(a) Uniform flow.

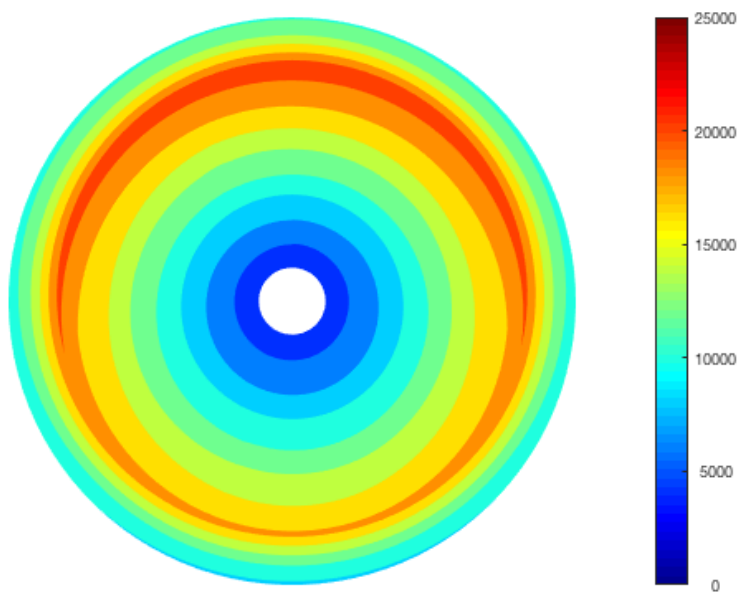

(b) Shear flow

Figure 5.9: Spanwise axial force $\left(\mathrm{N} \mathrm{m}^{-1}\right)$ variation along the blade during a rotation cycle in uniform and shear flow. The depth averaged flow velocity is $2 \mathrm{~ms}^{-1}$ in both cases.

attack on the blade in the top half of the rotation; see figure 5.7. Also the azimuthal asymmetry can be clearly identified with the angle of attack decreasing as the blade reaches the bottom of its rotation.

In both uniform and shear flow cases, the angle of attack along most of the span is in the range of $5^{\circ} \leq \alpha \leq 8^{\circ}$ which produces the optimum lift to drag ratio for the aerofoils used along the blade section; see figure 5.8. In the case of uniform flow, the angle of attack is azimuthally constant and hence only one line is shown which represents both top and bottom. In the case of shear flow, the angle of attack near the root is higher at both top and bottom positions compared to the uniform flow case due to higher axial flow velocity at this point; see figure 5.3.

The axial forces acting on the blade in uniform and shear flow have a similar magnitude 


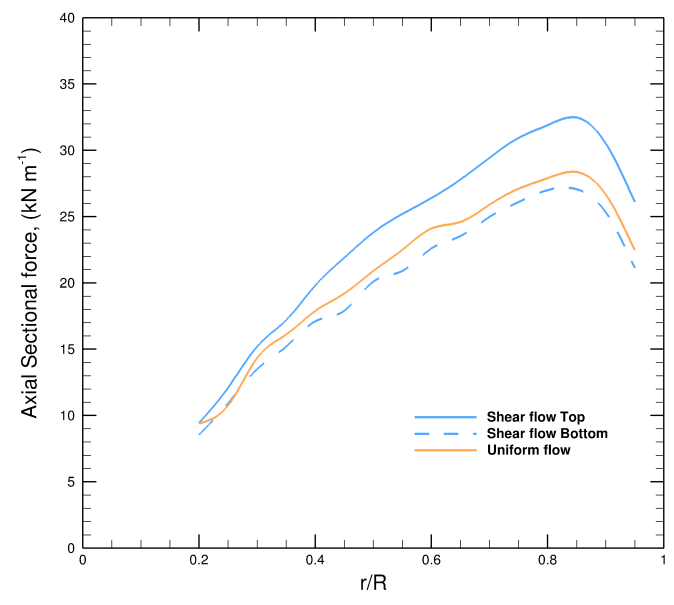

(a) Axial force

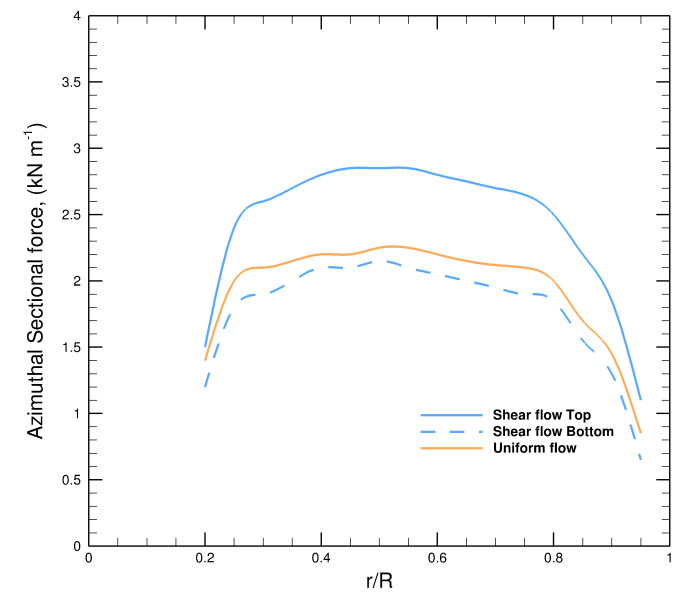

(b) Azimuthal force

Figure 5.10: Sectional force variation along the span of a turbine blade for uniform and shear flow, at the top and bottom position in a rotation cycle.

range but different spanwise distribution; see figure 5.9. The spanwise axial force variation is azimuthally constant in the uniform flow case, whereas there is an azimuthal asymmetry in the shear flow case, wherein the spanwise axial forces are higher in the top half of the blade rotation due to the higher flow speeds in that region. The higher forces correspond to the higher angles of attack observed in figure 5.8.

In both the uniform and shear flow cases, the axial force increases from the blade root towards the tip with the maximum force acting on the blade occurring at about $0.85 \mathrm{R}$, and then decreasing as the blade tip is approached; see figure 5.10a. The axial force at the blade top position is higher than that in the uniform flow whereas at the bottom position, it is lower than the uniform flow corresponding to the difference in vertical velocities in shear and uniform flow. The decrease in axial force at the blade tip is due to the three dimensional effects of the flow around the blade tip.

In the case of uniform flow, the azimuthal force is constant at top and bottom and hence a single line is shown in figure 5.10b. In the case of shear flow, the azimuthal force 
is higher at the top position than the bottom position resulting in fluctuations in power during a rotation cycle as observed in the previous section. Also it can be observed that the azimuthal force is nearly constant along the entire span, thus maximising the power obtained at this tip speed ratio.

\subsubsection{Wake comparison}

\subsubsection{Velocity}

The thrust on the rotor blades influences the stream-wise evolution of the axial flow velocity in the turbine wake. In the uniform flow case, the wake recovery is symmetric about the mid-height centre line while in the shear flow case, the wake recovery is faster in the lower region as compared to the upper half of the domain due to the lower forces experienced by the rotor in the bottom part of its rotation; see figure 5.11. The velocity deficit is negative in the bypass flow region since the bypass flow velocity is higher than the corresponding inlet velocity due to the presence of rotor.

The higher axial flow velocity in the upper half of the shear flow case results in a higher bypass flow in the wake in the top half compared to the bottom half; see figure 5.12.

The cross-stream variation in axial flow velocity at different locations in the near wake is presented in figure 5.13. A symmetric wake develops in the uniform flow case, whereas in the shear flow case, the wake has a vertical asymmetry as well as lateral asymmetry. The asymmetry is due to the interaction of the swirl, induced by the rotor, with the vertical shear in the flow.

\subsubsection{Turbulence intensity}

As discussed in the previous section, there is a higher bypass flow velocity at the top of the channel which results in a higher level of shear between the bypass flow and the wake. The 

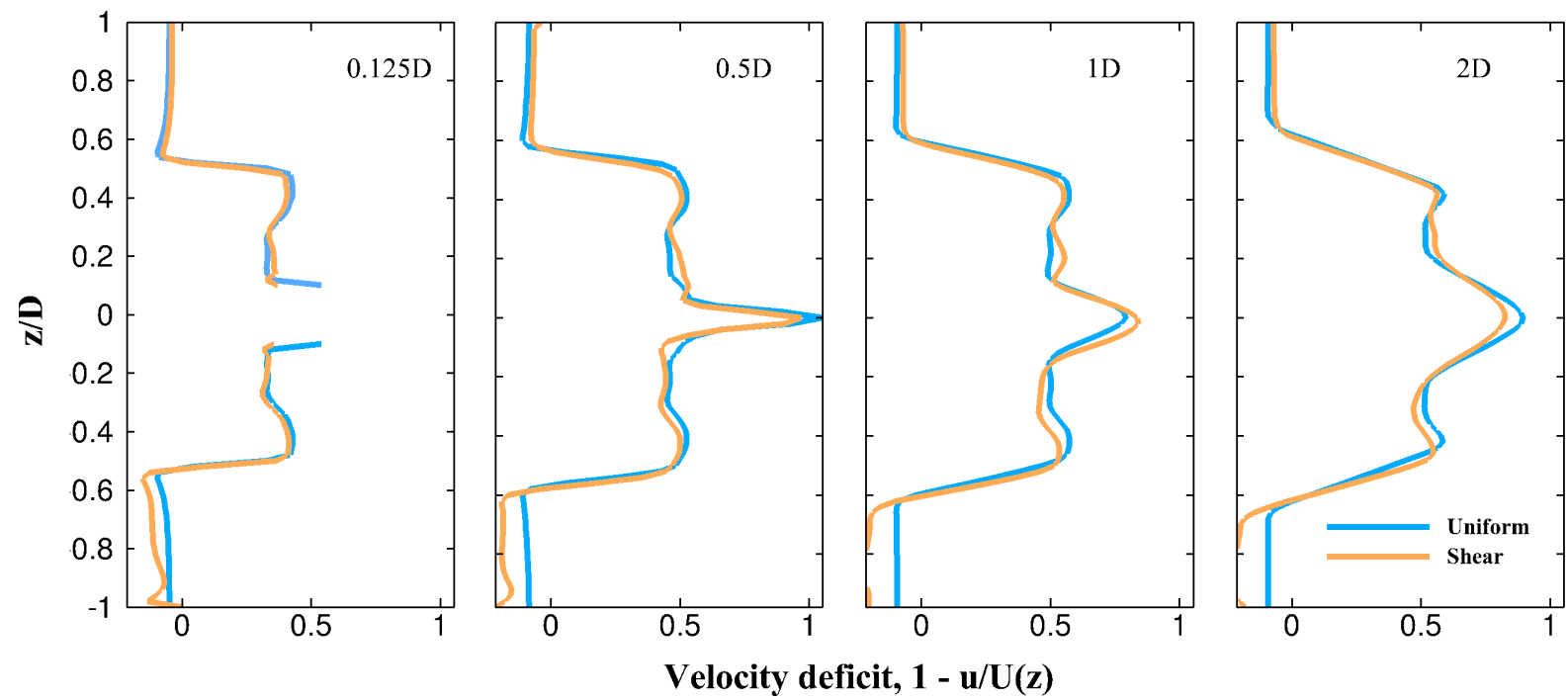

Figure 5.11: Normalised velocity deficit at different downstream locations in the near wake. $0.125 \mathrm{D}$ is the location of a potential support structure which will be discussed in the next chapter. In the shear flow case, the velocity is normalised based on the inlet shear profile.

higher shear leads to more mixing and more turbulence and thus leads to a higher level of turbulence intensity in the upper half than in the lower half of the channel in the shear flow case; see figure 5.14. The uniform flow case has a symmetric turbulence intensity about the rotor mid-plane.

The turbulence intensity is higher at the blade tips because the lower flow velocity immediately behind the rotor, and the higher bypass flow velocity cause mixing in the flow leading to higher turbulence. Hence closer to the rotor, $x / D=0.5$, the higher turbulence intensity zone is more concentrated as the mixing happens in a smaller zone due to high gradient in velocity whereas farther away from the rotor, $x / D=2$, the higher turbulence intensity zone is more dispersed as the mixing is more distributed by this point. 


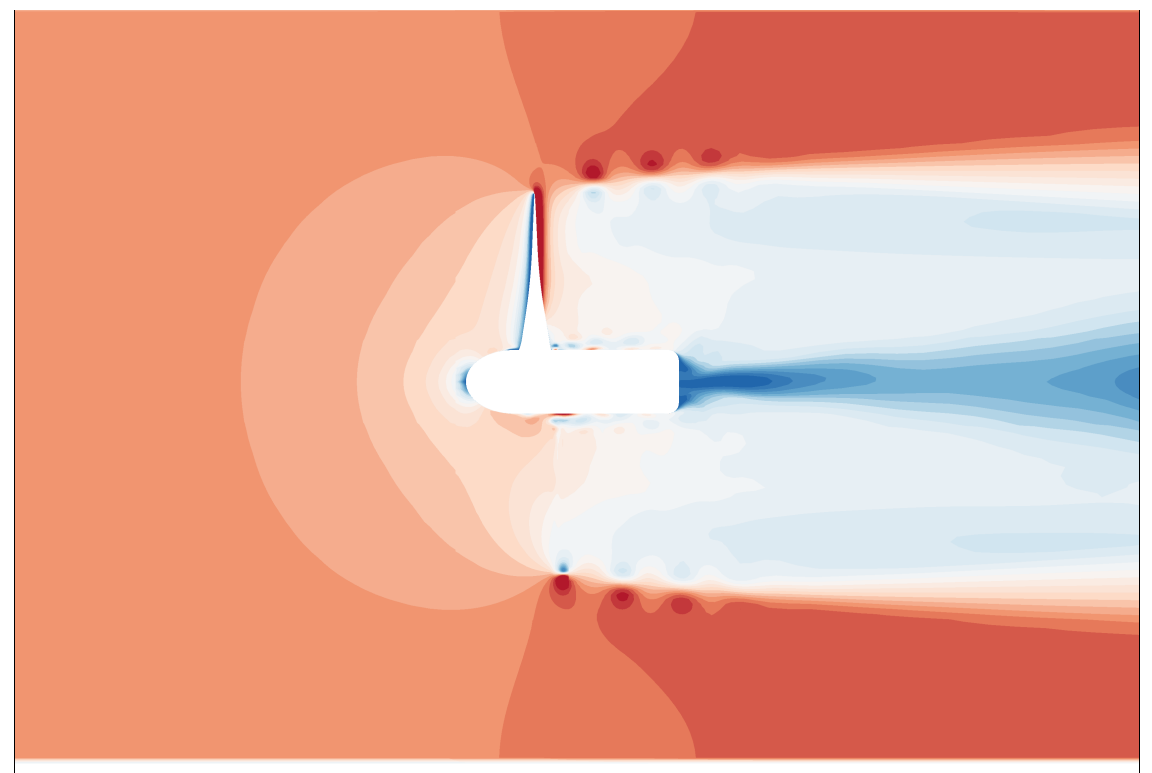

(a) Uniform flow

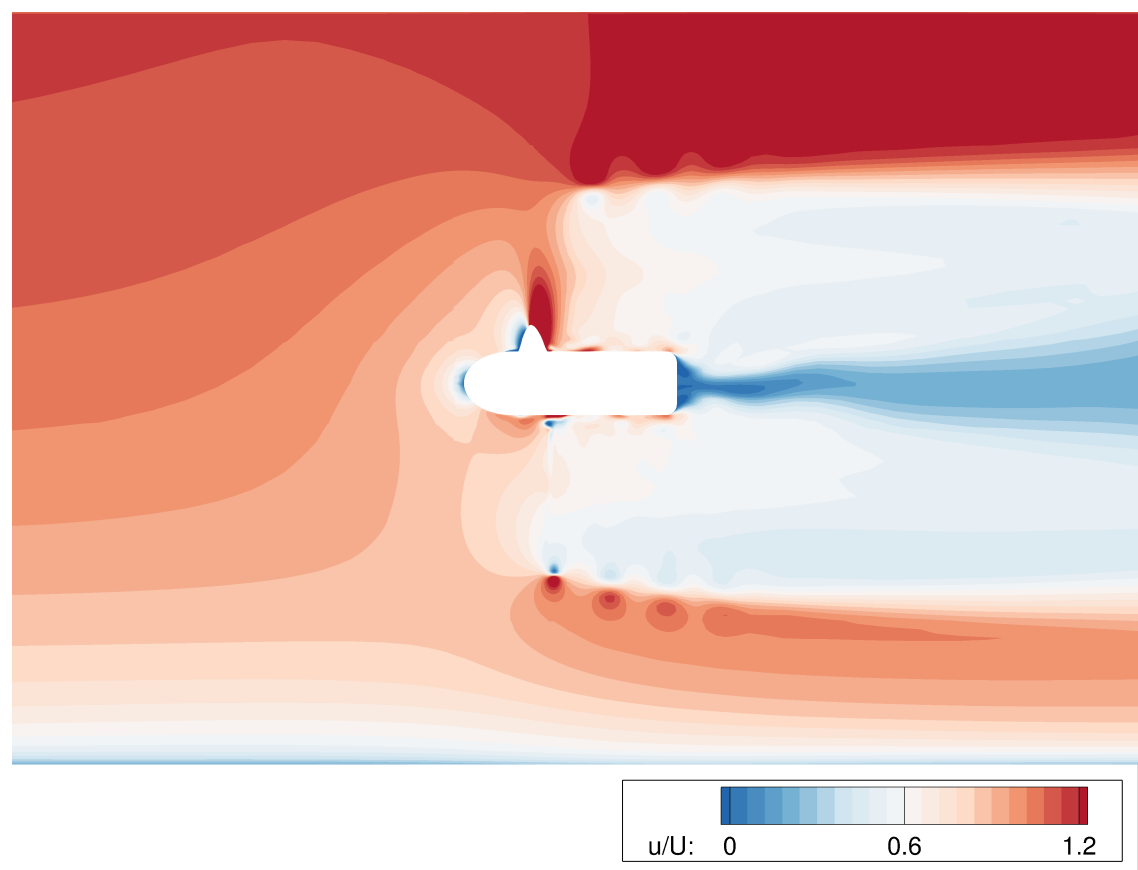

(b) Shear flow

Figure 5.12: Comparison of normalised axial velocity at lateral mid-section between the uniform flow case (a) and the shear flow case (b). In both the cases, the velocity is normalised with the depth averaged velocity of $2 \mathrm{~ms}^{-1}$. 

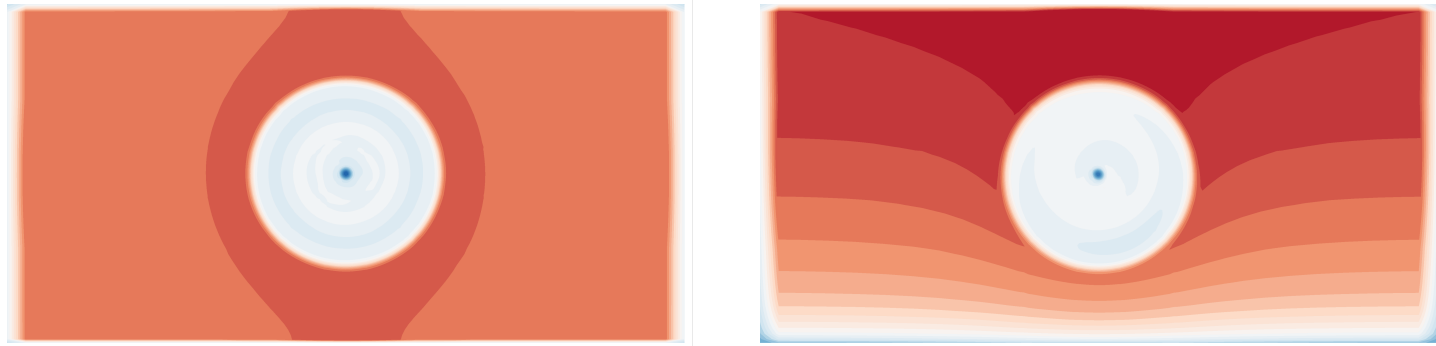

(a) $x=0.5 \mathrm{D}$
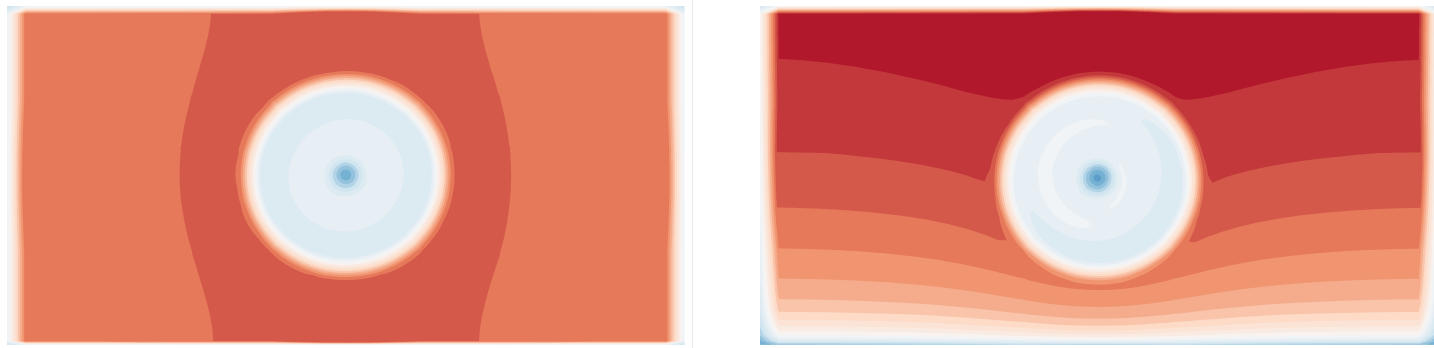

(b) $x=1 \mathrm{D}$
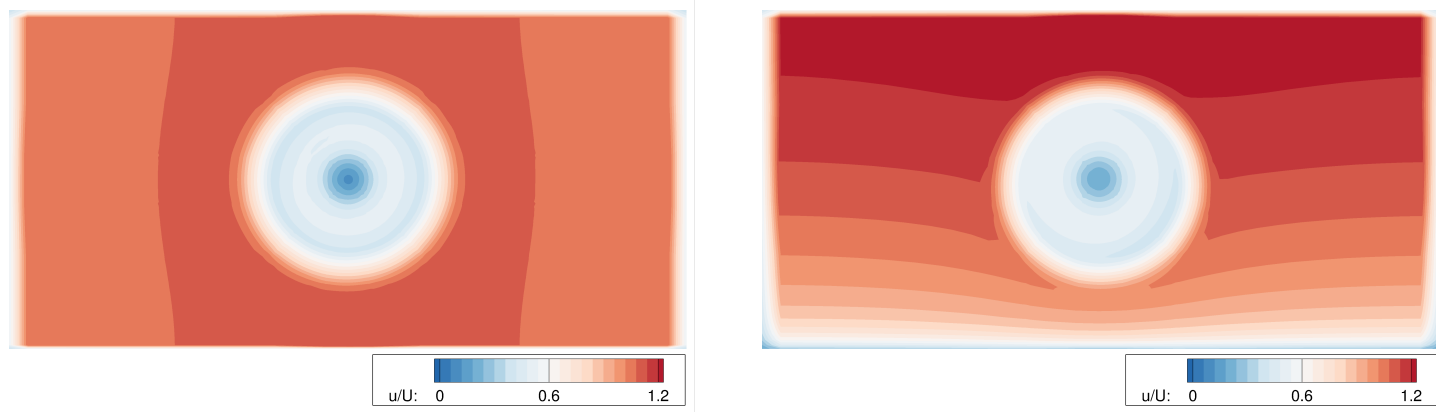

(c) $x=2 D$

Figure 5.13: Time-averaged cross-stream variation of axial velocity in the near wake for uniform (left) and shear (right) flow cases at three different locations downstream of the rotor plane. 


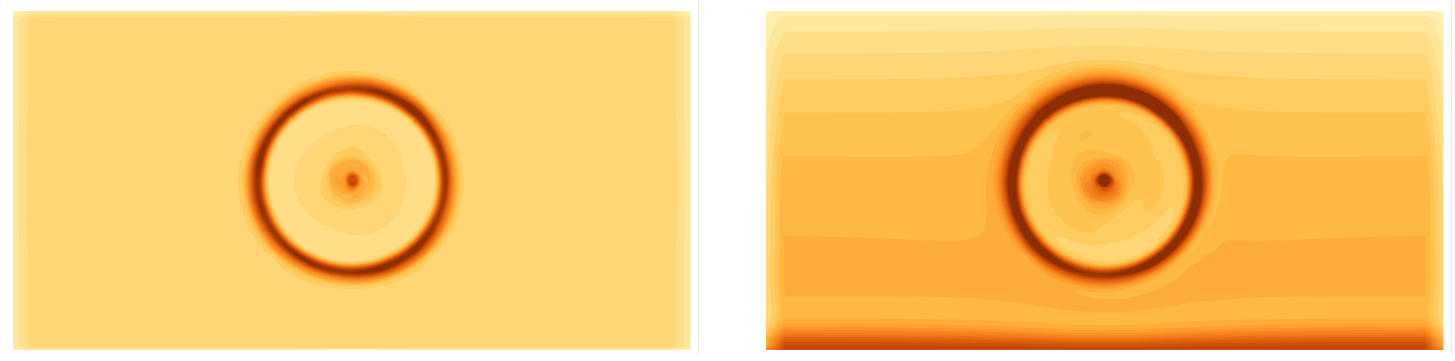

(a) $x=0.5 \mathrm{D}$
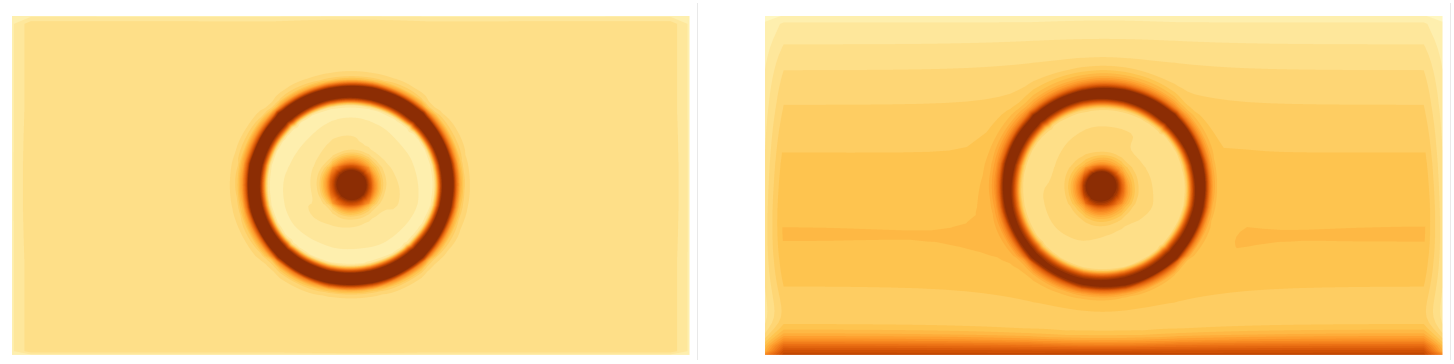

(b) $x=1 \mathrm{D}$
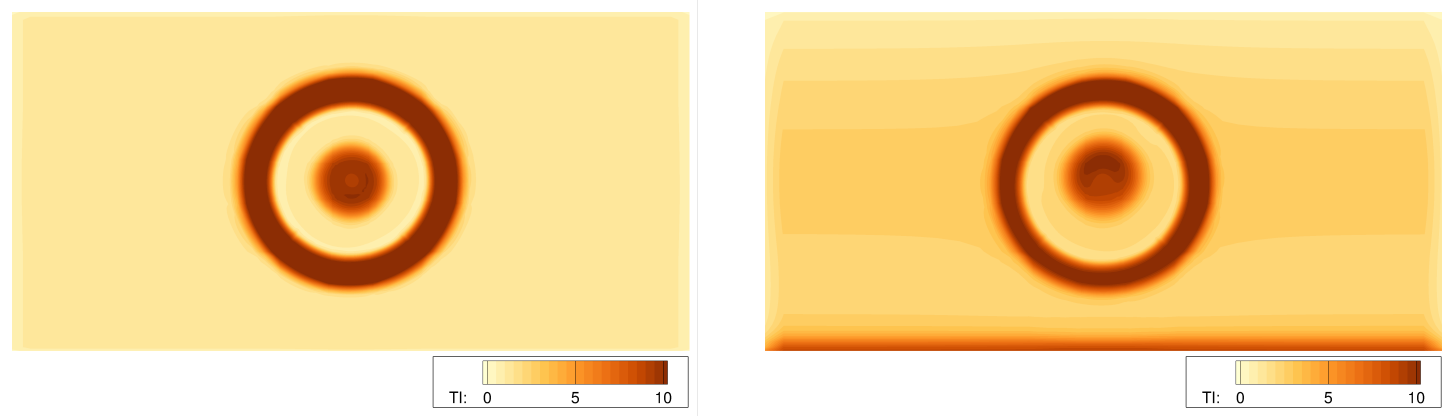

(c) $x=2 D$

Figure 5.14: Time-averaged cross-stream variation of turbulence intensity (TI) in the near wake for uniform (left) and shear (right) flow cases at three different locations downstream of the rotor plane. 


\subsection{Blade pitching in the power-capping zone}

The angular velocity of the rotor blades increases as the channel flow speed increases, resulting in an increase in the power output from the turbine. However, the generator inside a turbine is typically designed to operate up to a maximum angular velocity. Hence, beyond a certain flow velocity, the power output from the turbine is limited by maintaining a constant angular velocity and changing the blade pitch angle to feather. This strategy is known as pitch-to-feather power-capping. The maximum turbine power output is called the rated power and the threshold velocity is called the rated flow speed.

This commonly used pitch-to-feather strategy reduces the angle of attack on the turbine blades, thereby reducing the loads, and therefore the torque of the rotor. In this section, the influence of blade pitching on the turbine rotor forces and its wake is discussed. As an actual tidal channel has a sheared flow velocity profile, and so all the simulations in this section are performed using a shear flow profile.

Bahaj et al. (2007b) conducted towing tank experiments for different blade pitch angles and presented the variation in force coefficients with change in pitch angle. The data shows how the force coefficients peak at each tip speed ratio and a corresponding blade pitch angle. Vogel (2014) has studied the power control strategy using Blade Element Momentum (BEM) techniques. In this work, the change in power coefficients with tip speed ratio was studied for different blade pitch angles along with the power-control curve, thus obtaining the data points for the amount by which a blade should pitch at a corresponding tip speed ratio. There have been experimental and BEM studies on the influence of blade pitching but little research on the flow physics using blade-resolved CFD simulations. In this section, the loading on the blade due to blade pitching and its influence on the wake is studied in more detail. 


\subsubsection{Simulation strategy}

Above the rated flow speed, the turbine does not operate at the highest power coefficient $\left(\mathrm{C}_{P}\right)$ but instead follows a power control curve defined by the power-capping control strategy (Vogel, 2014). For pitch-to-feather this requires non-linearly increasing the pitch angle as the flow speed is increased above rated flow speed.

As discussed earlier, there are two approaches to simulating turbine rotation in blade resolved CFD simulations; through the Multiple Reference Frame (MRF) model and through mesh rotation. In the mesh rotation method, an inner local rotor mesh is physically rotated within a fixed outer domain, whereas in the MRF model, a Coriolis term is added to the Navier-Stokes equations in the inner domain, the rotor is held stationary and the approach flow rotated at the turbine. The MRF approach is steady and cannot capture the entire wake (unless the MRF exit plane extends to the outflow boundary which precludes the inclusion of a support structure).

Designing a pitch-to-feather strategy requires the determination of the pitch angle as a function of flow speed, that is required to achieve a particular power coefficient at a designated tip-speed-ratio, $\lambda$. The procedure to get the operating points in the powercapping region involves simulating multiple tip speed ratios at each blade pitch angle. The intersection points between the turbine power control curve and the $C_{P}$ curves define the turbine operating locus; see figure 5.15. To achieve this computationally, the initial $C_{P}$ curves were first obtained at discrete tip speed ratios using the MRF model which provides a quick estimate of the force coefficients. The intersection point between the power control curve and the $C_{P}$ curves was then determined from the slope, $\mathrm{d} C_{P} / \mathrm{d} \lambda$, of the closest points on either side of the power control curve.

At each of the operating points, one for each feather angle, the mesh motion CFD model was then used to conduct unsteady simulations which allowed analysis of the wake 


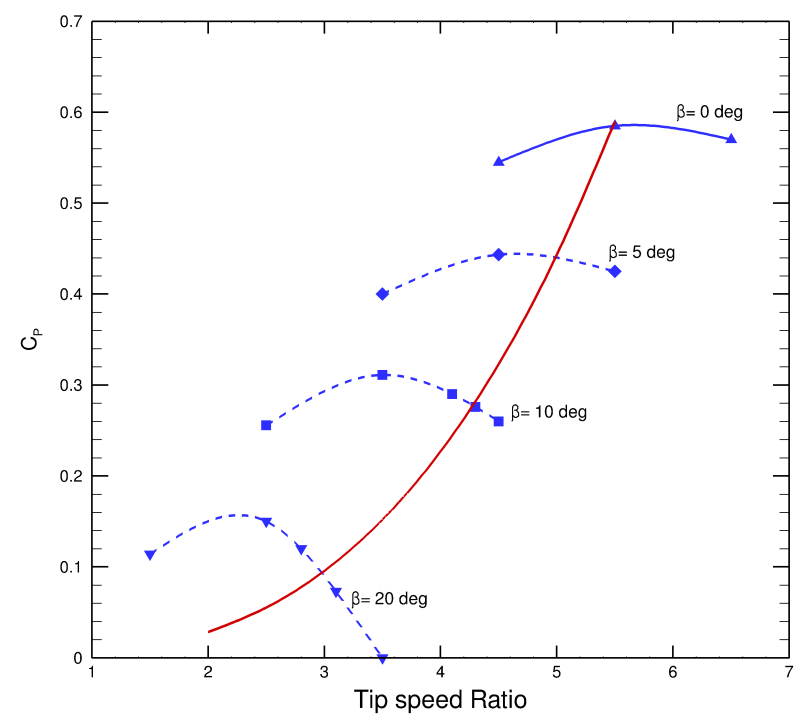

Figure 5.15: $\mathrm{C}_{P}$ curves for four different blade pitch angles (blue lines), $\beta ; 0^{\circ}, 5^{\circ}, 10^{\circ}$ and $20^{\circ}$. The intersection points between the $\mathrm{C}_{P}$ curves and power control line (red) are the operating points.

properties as well as the unsteady forces on the turbine. The power and thrust coefficients at these different operating points were determined and their correlation with the velocity deficit in the wake was studied. The sectional forces along the length of the blade were evaluated and the radial position at which the maximum load occurs was studied for different blade pitch angles. The velocity profiles at different locations in near wake were determined at different blade pitch angles, and the velocity profile near a hypothetical support structure was also determined. This gives an estimate of the velocity and pressure distribution just ahead of the support structure. This analysis provides valuable results for the design of a turbine blade as the force distribution along the blade changes for different blade pitch angles. These results are presented in the following sections. 
Table 5.1: Rotor force coefficients for three different blade pitch angles and flow speeds

\begin{tabular}{|c|c|c|l|l|}
\hline Pitch angle & TSR & Channel Velocity, $\mathrm{ms}^{-1}$ & $C_{P}$ & $C_{T}$ \\
\hline $0^{\circ}$ & 5.5 & 2.37 & 0.622 & 0.984 \\
\hline $10^{\circ}$ & 4.3 & 2.96 & 0.308 & 0.372 \\
\hline $20^{\circ}$ & 3.1 & 4.5 & 0.085 & 0.112 \\
\hline
\end{tabular}

\subsubsection{Power and thrust coefficients of the rotor}

Three different points were analysed in the power-capping regime as representative of the channel velocities in the turbine operating regime. Unsteady flow simulations were carried out at these corresponding tip speed ratios. These three tip speed ratios correspond to the pitch angles of $0^{\circ}, 10^{\circ}$ and $20^{\circ}$, and the power and thrust coefficients at these blade pitch angles are presented in table 5.1.

The power coefficient drops by about $50 \%$ between $0^{\circ}$ and $10^{\circ}$ cases, and by about $85 \%$ between $0^{\circ}$ and $20^{\circ}$ cases. There is a non-linear drop in power coefficient such that the turbine power output remains approximately at the rated power of $1 \mathrm{MW}$ in the powercapping zone; see figure 5.16. The thrust coefficient drops by about $60 \%$ between $0^{\circ}$ and $10^{\circ}$ cases, and by about $88 \%$ between $0^{\circ}$ and $20^{\circ}$ cases. The drop in the thrust coefficient is non-linear with velocity due to the non-linear change in the angle of attack on the blades when they are pitched which is explained in detail in the next section. The rotor thrust itself drops by about $40 \%$ between $0^{\circ}$ and $10^{\circ}$ cases, and by about $57 \%$ between $0^{\circ}$ and $20^{\circ}$ cases; see figure 5.16 .

The axial root blade bending moment on a single blade drops by about $64 \%$ between $0^{\circ}$ and $10^{\circ}$ cases, and by about $90 \%$ between $0^{\circ}$ and $20^{\circ}$ cases; see figure 5.16 , thus experiencing significantly lower stress on the blades in the power-capping zone.

During the rotation of a turbine blade in a shear flow, the blade moves through different 


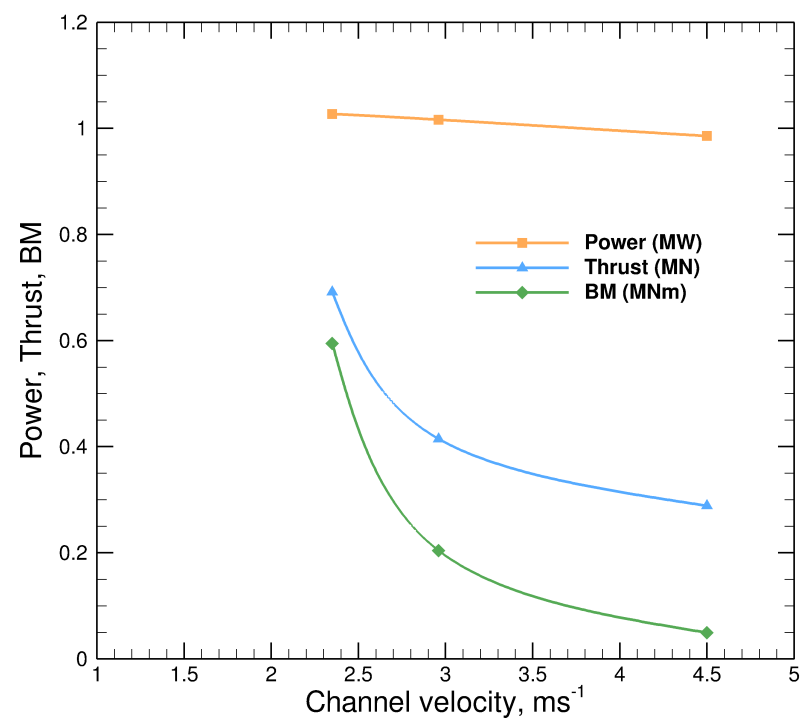

Figure 5.16: Variation in power and thrust on the turbine rotor with channel flow speed. Each channel flow velocity corresponds to a different blade pitch angle of $0^{\circ}\left(2.37 \mathrm{~ms}^{-1}\right)$, $10^{\circ}\left(2.96 \mathrm{~ms}^{-1}\right)$ and $20^{\circ}\left(4.5 \mathrm{~ms}^{-1}\right)$. Also plotted is the axial root bending moment (BM) on a single blade for the three different blade pitch angles.

velocity zones resulting in periodic forces on the blade; see figure 5.17. The relative fluctuations in the normalised blade force coefficients increase with increasing blade pitch angle as the angle of attack on the blade varies more during a rotation in the higher blade pitch angle case which will be discussed in the next section.

\subsubsection{Blade forces}

In the previous section, the variation in the integrated forces on a blade during its rotation was discussed. In this section, the force variation along the span of the blade and the angle of attack on the blade are analysed.

The total approach angle, $\phi$, along the span of the blade during its rotation cycle is presented in figure 5.18. Vertical asymmetry can be observed with higher approach angles in the top half compared to the lower half of the cycle. As the blade pitch angle, $\beta$, is 


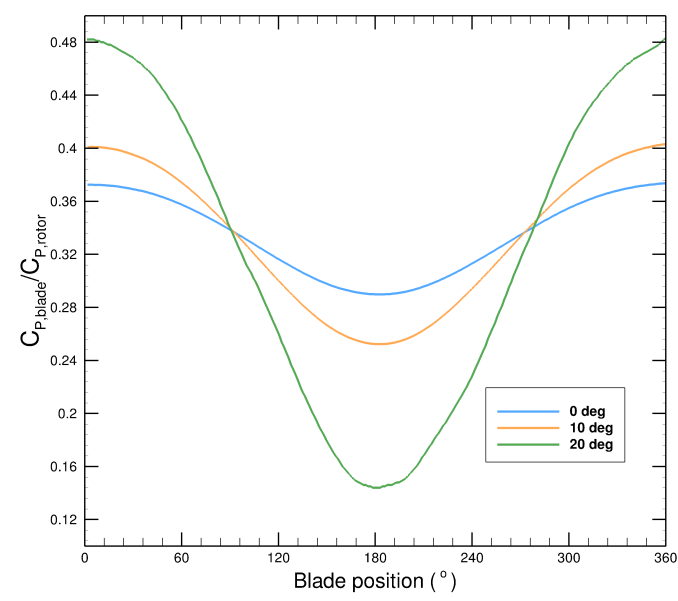

(a) Normalised power coefficient

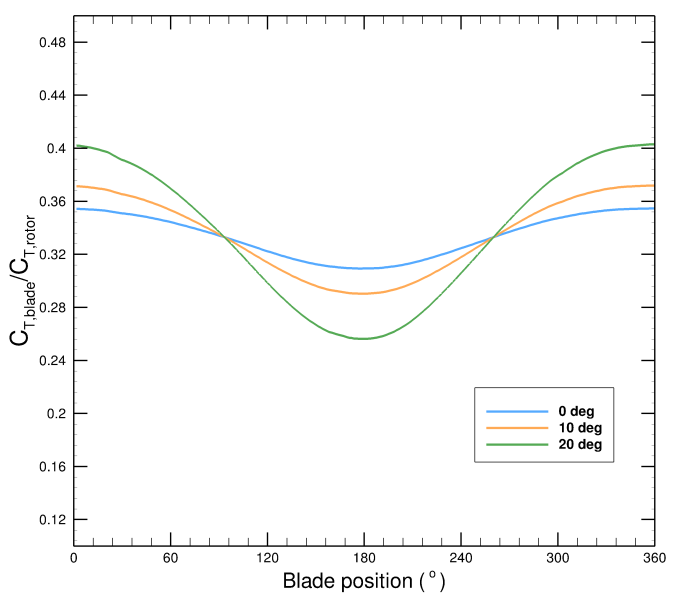

(b) Normalised thrust coefficient

Figure 5.17: Blade power (a) and thrust (b) coefficients normalised with respect to the integrated rotor force coefficients for three different blade pitch angles during one rotation cycle.

increased at higher flow velocities while maintaining the blade angular velocity constant, there is a decrease in the rotor thrust as discussed in the previous section, which results in an increase in the axial velocity through the rotor. This results in an increase in the approach angle with increasing blade pitch angle.

The angle of attack, $\alpha(=\phi-\beta)$, on the blade for the three different blade pitch angles is presented in figure 5.19. In the case of $0^{\circ}$ blade pitch angle, angle of attack is generally in the range of $5^{\circ}-8^{\circ}$ which corresponds to the optimum lift to drag ratio for the aerofoil sections used in the blade; see figure 5.20. The angle of attack is higher at the top position than the bottom position due to the higher axial flow speed at the top. Also the variation in angle of attack along the span is much lower compared to the other two blade pitch angles. This results in the blade with $0^{\circ}$ pitch, achieving an optimum torque along most of the blade span as per the design requirement.

As the blades are pitched at higher flow velocities, the angle of attack on the blade decreases even though the approach angle on the blade increases as discussed earlier; see 


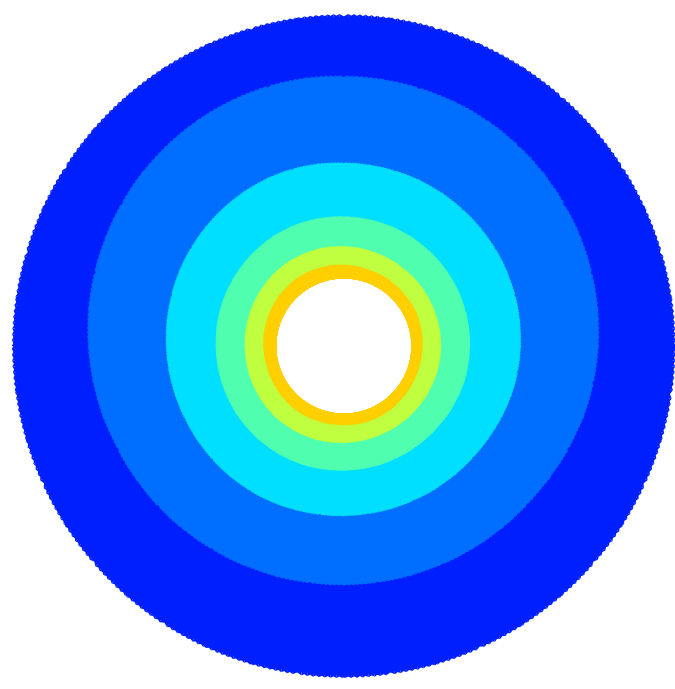

(a) $0^{\circ}$ pitch

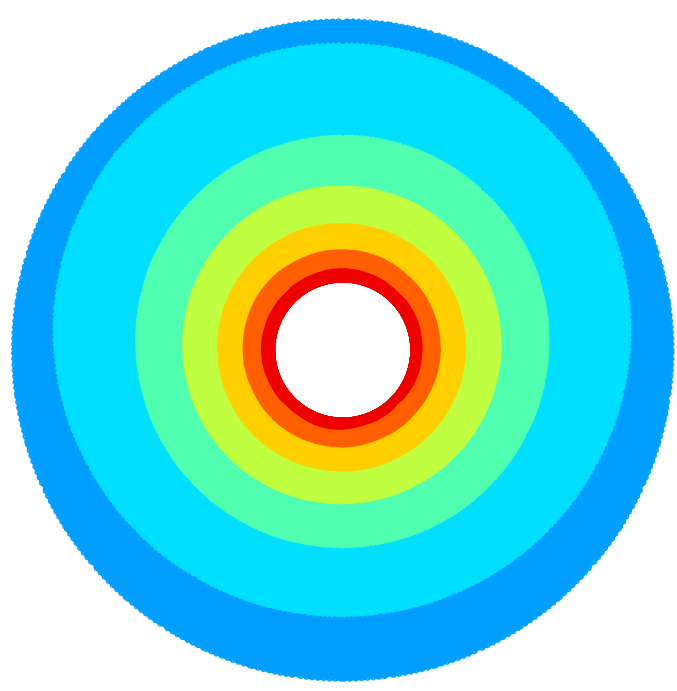

(b) $10^{\circ}$ pitch

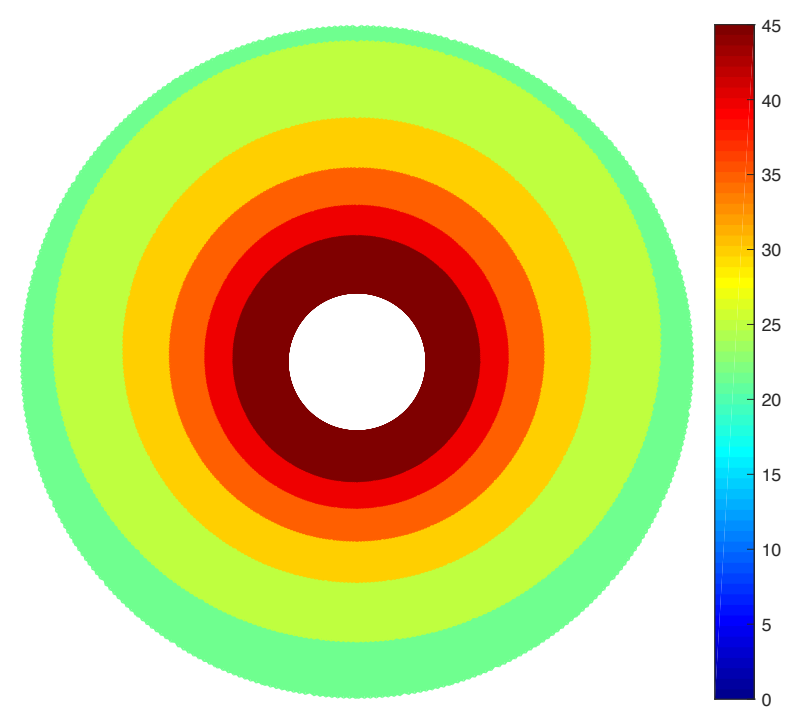

(c) $20^{\circ}$ pitch

Figure 5.18: Spanwise variation (rotor swept area) of approach angle, $\phi^{\circ}$, on a turbine blade for three different blade pitch angles, (a) $0^{\circ}$, (b) $10^{\circ}$ and (c) $20^{\circ}$ during a rotation cycle. 


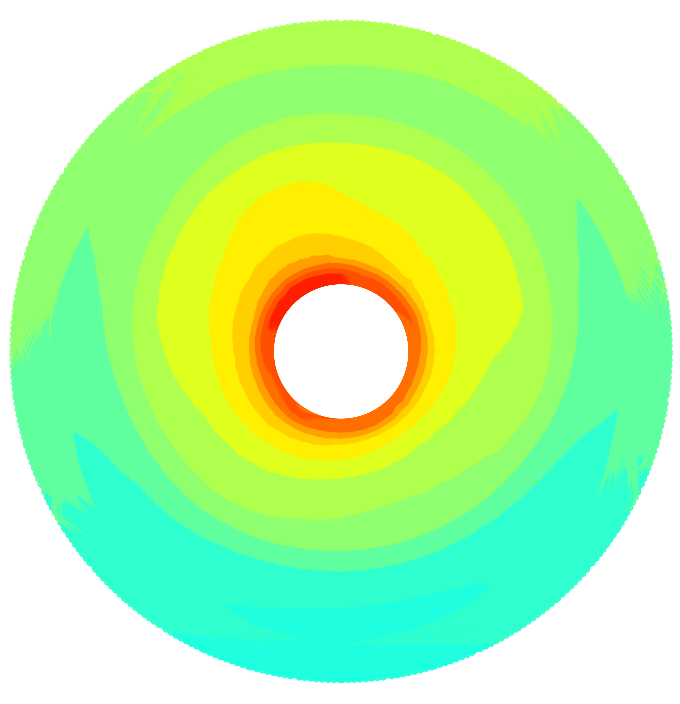

(a) $0^{\circ}$ pitch.

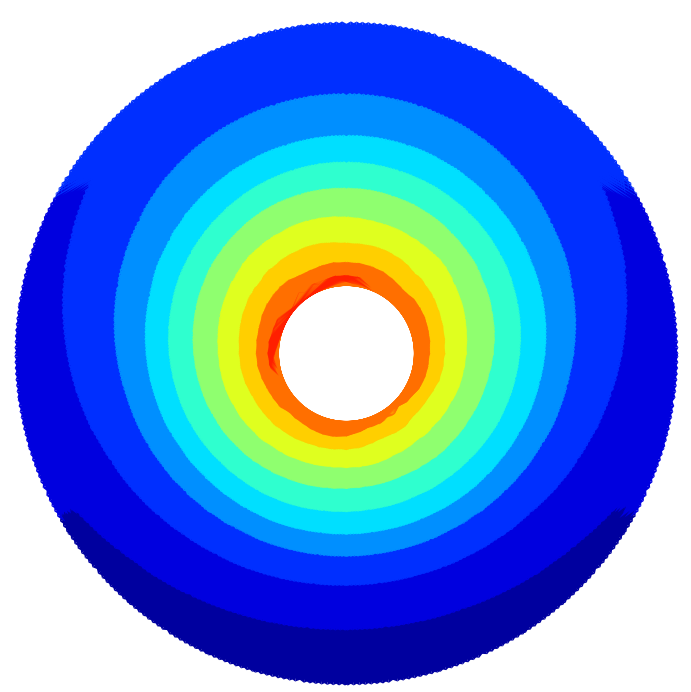

(b) $10^{\circ}$ pitch

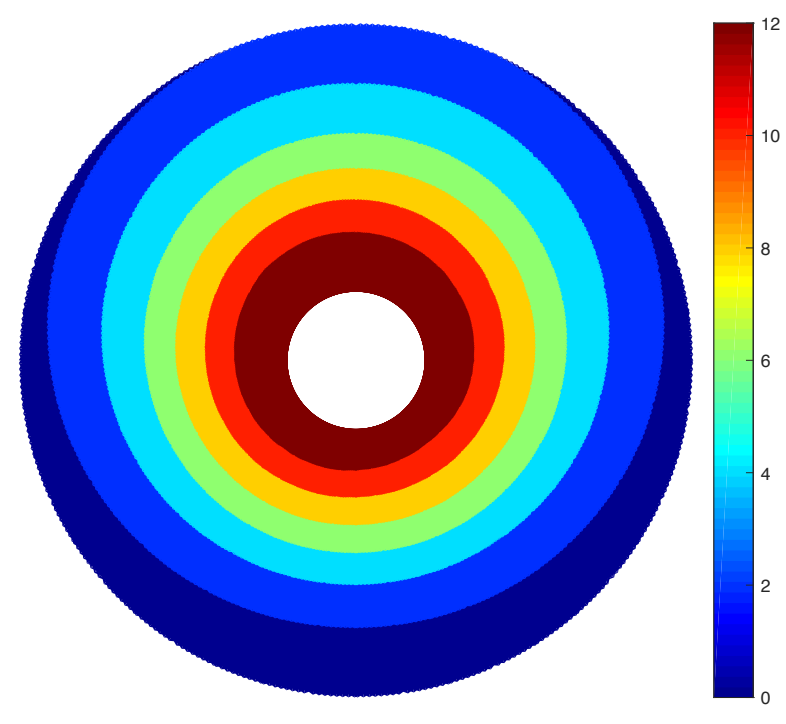

(c) $20^{\circ}$ pitch

Figure 5.19: Spanwise variation (rotor swept area) of angle of attack, $\alpha^{\circ}$, on a turbine blade for three different blade pitch angles, (a) $0^{\circ}$, (b) $10^{\circ}$ and (c) $20^{\circ}$ during a rotation cycle. 


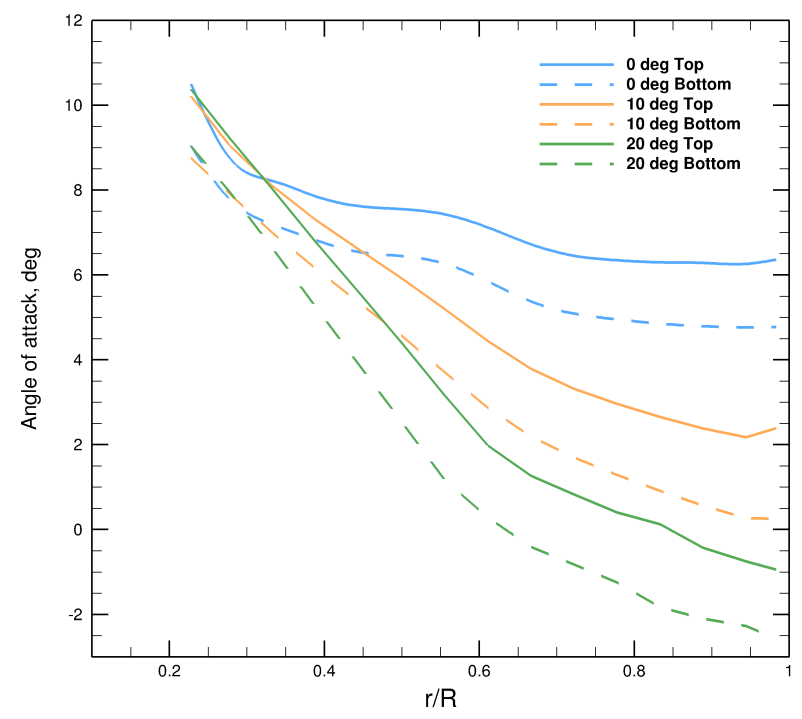

Figure 5.20: Angle of attack variation along the span of a turbine blade for three different blade pitch angles at the top (solid) and bottom (dashed) position in a rotation cycle.

figure 5.20. The drop in the angle of attack along the blade span is greater as the blade pitch angle is increased so that the rotor power coefficient decreases to maintain constant power in the power-capping zone.

In the case of $20^{\circ}$ blade pitch angle the angle of attack is negative at the tip of the blade when it is at the bottom position in its rotation cycle; see figure 5.20. The sectional pressure plot confirms this as the stagnation point moves towards the suction surface of the aerofoil indicating a negative angle of attack; see figure 5.21b.

The axial force variation on a blade for three different blade pitch angles is presented in figure 5.22. The axial force is higher in the top half of the cycle than the bottom half due to the higher angles of attack near the top resulting from the higher shear flow velocity as discussed earlier.

The axial force drops quite significantly with change in the blade pitch angle; see figure 5.22. Note that the contour levels are different for the three figures. Also the point of maximum axial force along the blade span moves from the blade tip towards the root 


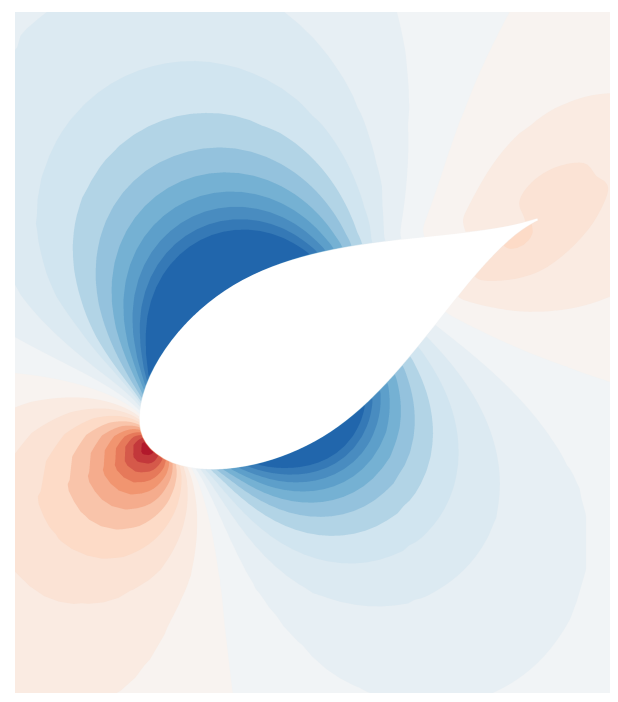

(a) Section at $0.5 \mathrm{R}$

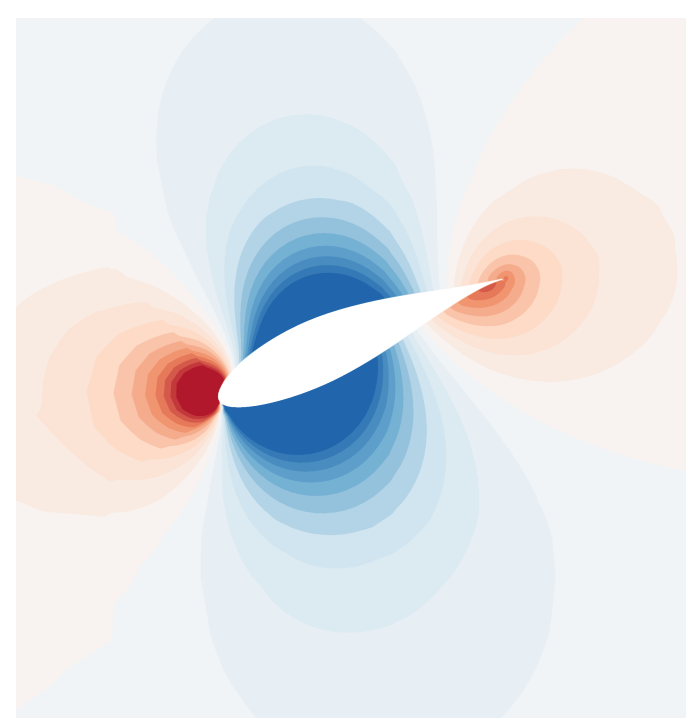

(b) Section at $0.95 \mathrm{R}$

Figure 5.21: Sectional plots of pressure distribution on the blade at $180^{\circ}$ position (bottom rotational position) at (a) $r / R=0.5$ and (b) $r / R=0.95$ to observe the location of stagnation point. Stagnation point moves from the lower surface to upper surface of the aerofoil indicating a negative angle of attack towards the tip of the blade. 


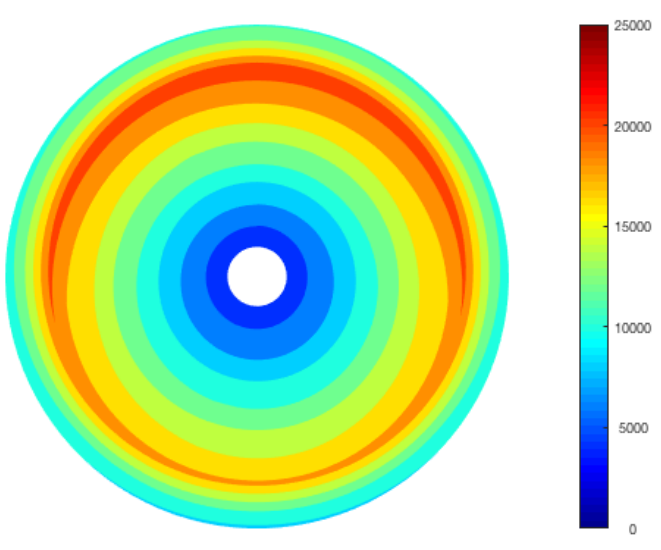

(a) $0^{\circ}$ pitch.

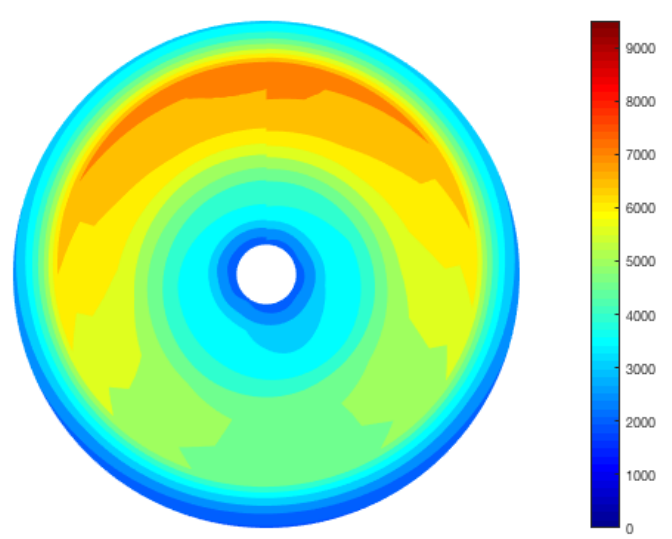

(b) $10^{\circ}$ pitch

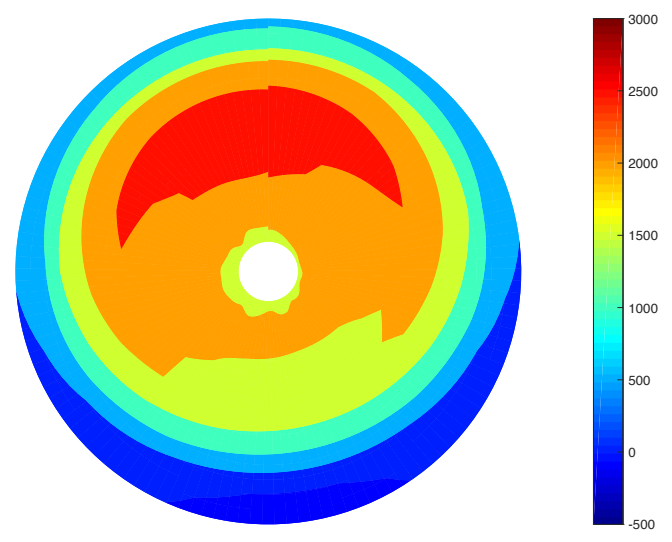

(c) $20^{\circ}$ pitch

Figure 5.22: Spanwise axial force, in $\mathrm{N}$, variation on a blade for three different blade pitch angles, (a) $0^{\circ}$, (b) $10^{\circ}$ and (c) $20^{\circ}$ in shear flow. Notice that the contour range is different in each case. 


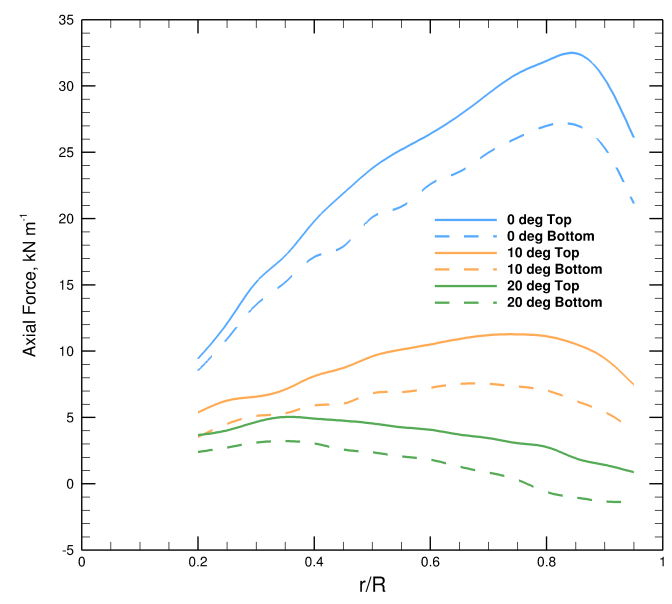

(a) Axial force

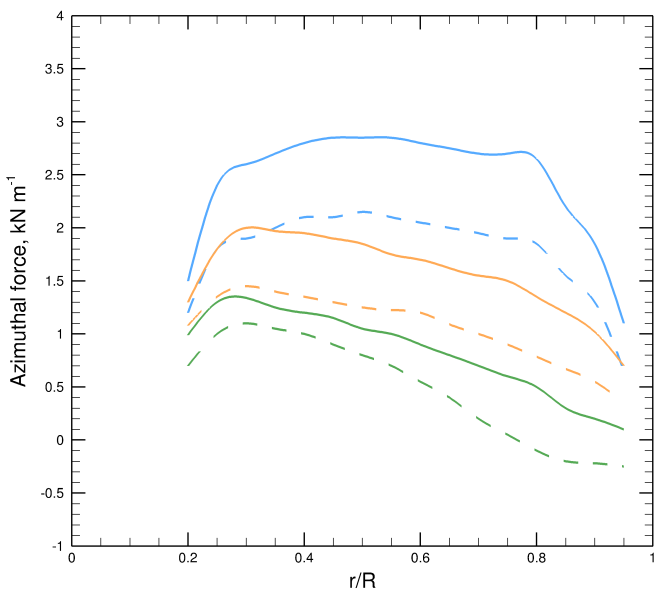

(b) Azimuthal force

Figure 5.23: Sectional force variation along the span of a turbine blade for three different blade pitch angles, $0^{\circ}, 10^{\circ}$ and $20^{\circ}$, at the top and bottom position in a rotation cycle.

with increasing blade pitch angle; see figure 5.23a. In the case of $0^{\circ}$ blade pitch angle, the axial force along the span increases gradually until $r / R=0.85$, and then drops towards the tip; see figure 5.23a. In contrast as the blade is pitched to $10^{\circ}$, the force distribution is more uniform towards the central portion of the blade before dropping off towards the tip. In the case of $20^{\circ}$ blade pitch angle, the axial force is higher near the root at $r / R=0.33$ and gradually drops off over the span of the blade. This corresponds to the change in the angle of attack observed earlier wherein the angle of attack drops off faster towards the blade tip at higher blade pitch angles thus causing a reduction in forces.

In the case of sectional azimuthal force for $0^{\circ}$ blade pitch angle, it is almost constant at the mid-section of the blade thus attaining maximum torque and power at the design tip speed ratio; see figure 5.23b. As the blade pitch angle increases, the decrease in the angle of attack causes a decrease in the azimuthal force and hence the torque such that the power output remains constant in the power-capping zone.

In the $20^{\circ}$ blade pitch angle case, the axial and azimuthal forces near the tip of the 
blade are negative at the bottom of the blade rotation. This corresponds to the negative angle of attack on the aerofoil section near the tip of the blade when it is at the bottom position, as discussed earlier.

\subsubsection{Wake comparison}

In the previous section, the variation of turbine rotor thrust with varying blade pitch angle was discussed. The turbine thrust has a direct effect on the flow velocity in the wake which will be discussed in this section.

The velocity deficit at different locations in the near wake for different blade pitch angles is presented in figure 5.24. In all the cases, the velocity deficit is negative in the bypass flow zone as the thrust from the rotor increases the bypass flow velocity. Hence the $0^{\circ}$ blade pitch angle case with higher thrust has higher bypass flow velocity resulting in higher negative velocity deficit. The velocity recovery is faster as the blade pitch angle is increased, as the rotor presents less opposing thrust to the flow which results in a smaller reduction in flow velocity as it passes through the rotor; see figure 5.25.

At a blade pitch angle of $0^{\circ}$, the thrust on the turbine is higher which leads to a lower wake velocity and higher bypass flow velocity as compared to the $10^{\circ}$ blade pitch angle case. This leads to a higher shear between the wake and bypass flow in the $0^{\circ}$ case as compared to the $10^{\circ}$ case which results in higher turbulence intensity in the $0^{\circ}$ case; see figure 5.26.

The wake width is also reduced with increasing blade pitch angle, as reduced rotor thrust results in a higher mass flow through the rotor swept area, which in-turn results in a narrower streamtube around the rotor.

To identify the blade tip vortices, the $Q$ criterion (Hunt et al., 1988) is used. The $Q$ 

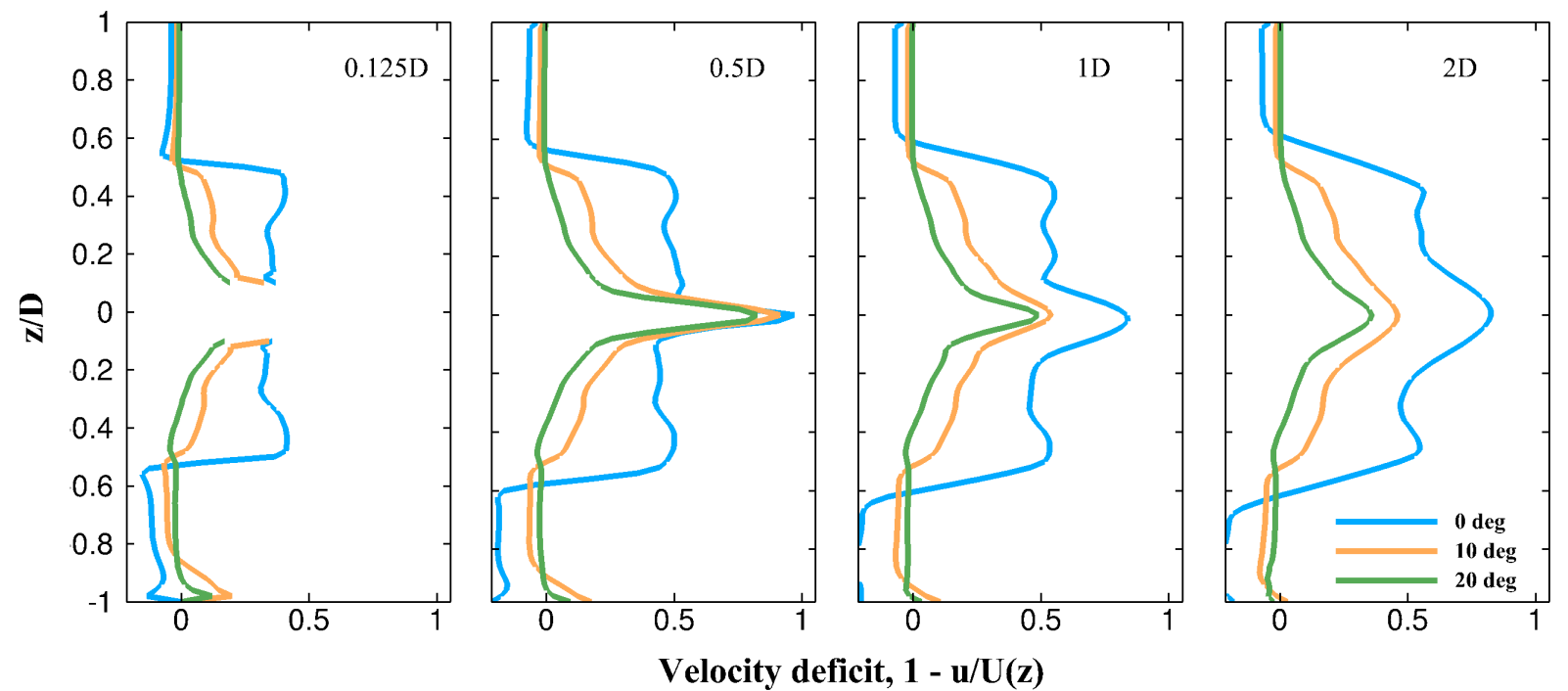

Figure 5.24: Vertical variation in normalised velocity deficit at four different streamwise locations, $0.125 \mathrm{D}, 0.5 \mathrm{D}, 1 \mathrm{D}$ and $2 \mathrm{D}$ in the near wake for three different blade pitch angles, $0^{\circ}, 10^{\circ}$ and $20^{\circ}$. The velocities are normalised with respect to the inlet shear profile. $0.125 \mathrm{D}$ is the location of a potential support structure which will be discussed in the next chapter. 


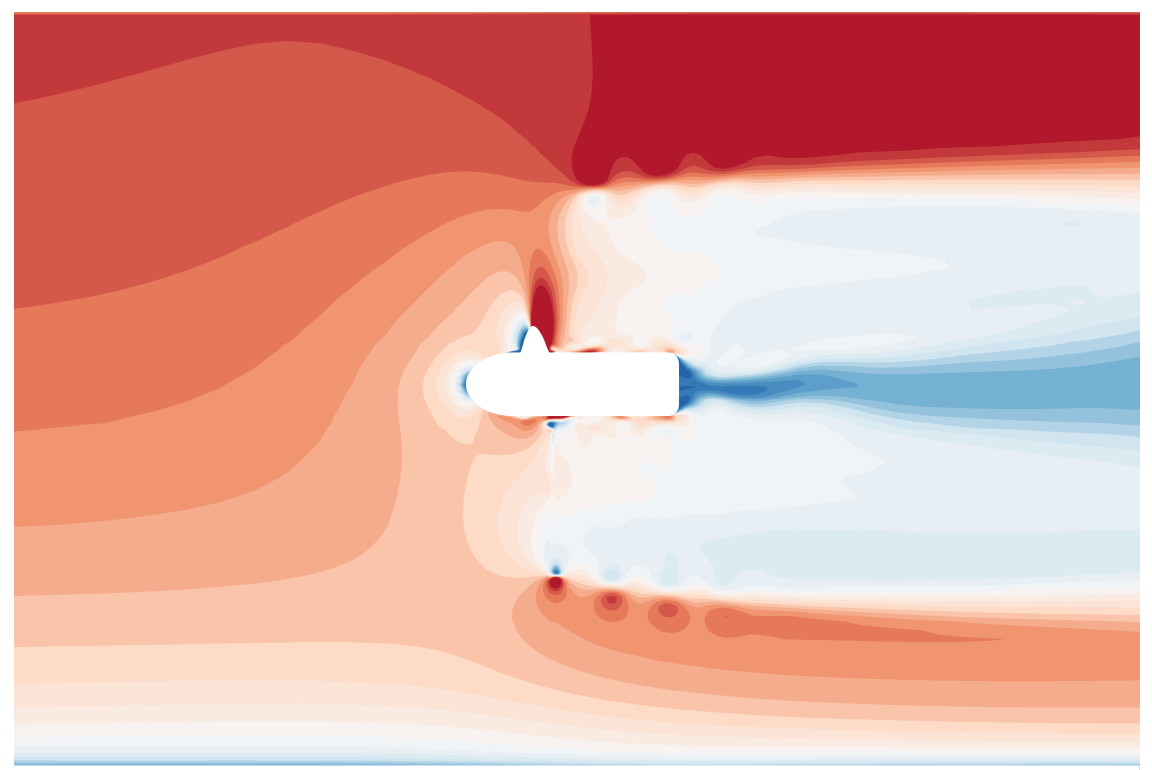

(a) $0^{\circ}$ pitch

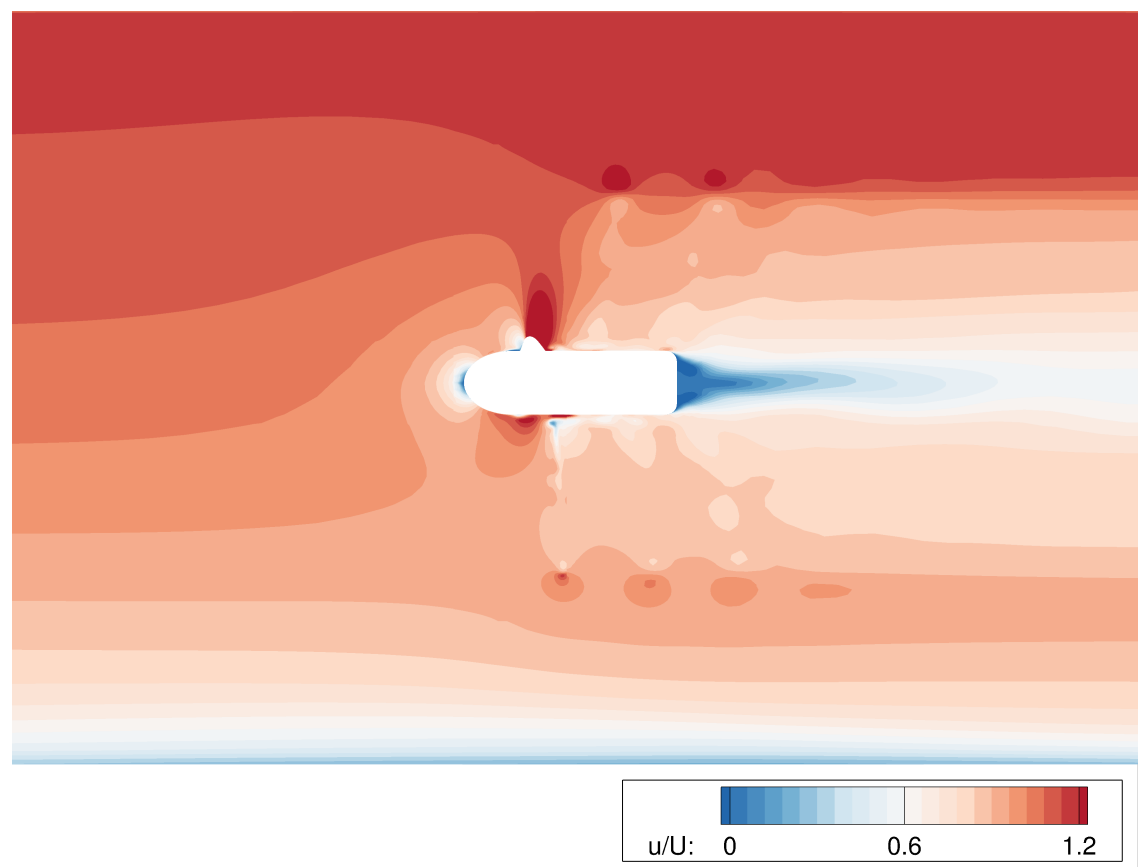

(b) $10^{\circ}$ pitch

Figure 5.25: Variation in normalised axial velocity at lateral mid-section for two different blade pitch angles, $0^{\circ}$ and $10^{\circ}$. The velocities are normalised with depth averaged velocity. 

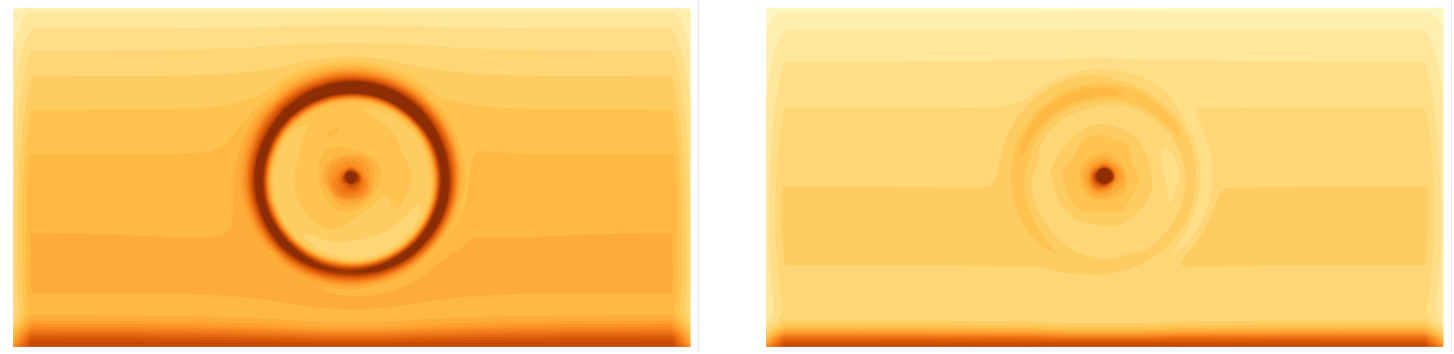

(a) Section at $0.5 \mathrm{D}$ downstream
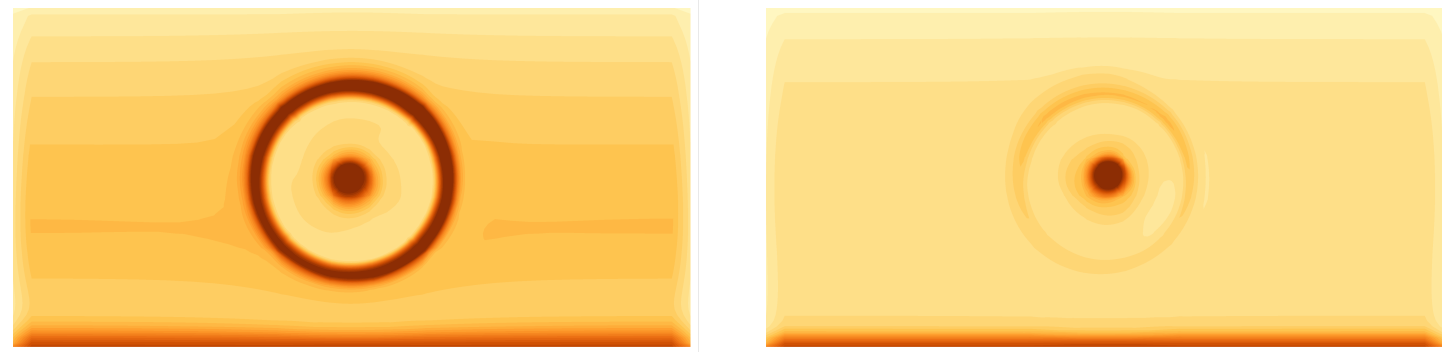

(b)Section at 1D downstream
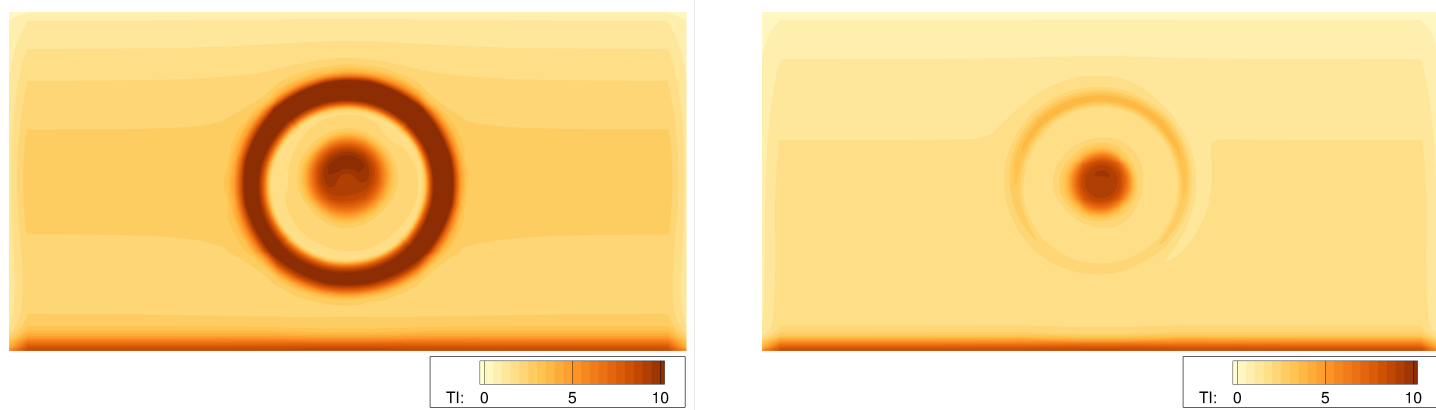

(c) Section at 2D downstream

Figure 5.26: Cross-stream variation in turbulence intensity (TI) at three different streamwise locations, (a) $0.5 \mathrm{D}$, (b) $1 \mathrm{D}$ and (c) $2 \mathrm{D}$ in the wake downstream for $0^{\circ}$ pitch (left) and $10^{\circ}$ pitch (right) cases. 


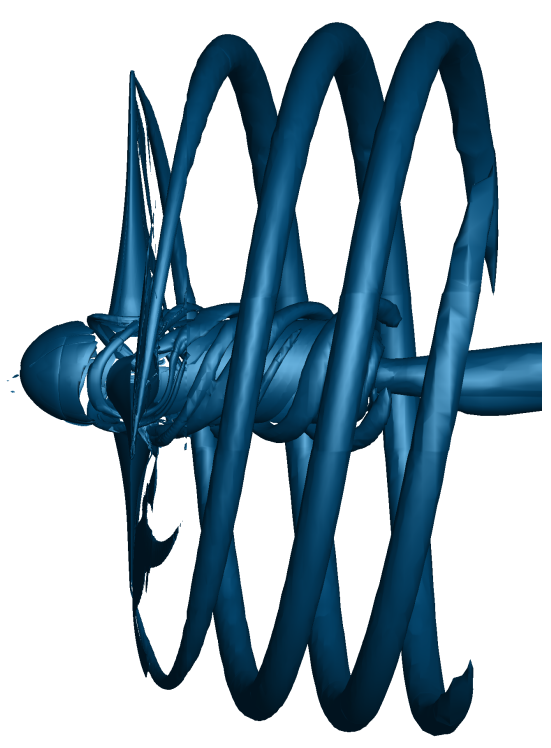

(a) $0^{\circ}$ pitch.

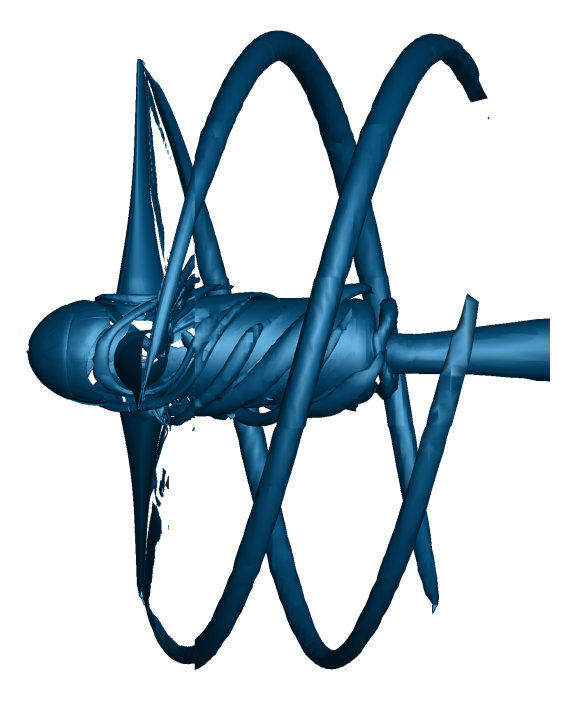

(b) $10^{\circ}$ pitch

Figure 5.27: Q criterion for two different blade pitch angles, (a) $0^{\circ}$ and (b) $10^{\circ}$ in shear flow; $\mathrm{Q}=0.2$.

criterion is defined by

$$
Q=\frac{1}{2}\left(\|\underline{\Omega}\|^{2}-\|\underline{S}\|^{2}\right)
$$

where $\Omega$ is the vorticity tensor and $S$ is the strain rate tensor. The $Q$ criterion is used over the vorticity magnitude to isolate vortex structures from the velocity shear. Hence a positive $Q$ denotes regions where the rotation rate dominates the strain rate. The $Q$ criterion for blade pitch angles of $0^{\circ}$ and $10^{\circ}$ is presented in figure 5.27. The lower wake velocity in the $0^{\circ}$ blade pitch angle case leads to slower transport of the tip vortex in the wake which results in a shorter helix pitch compared to the $10^{\circ}$ case. The width of the wake in the $10^{\circ}$ case can be noticed to be smaller than that in the $0^{\circ}$ case as discussed earlier. It can also be observed that the strength of tip vortices is much lower as blade pitch angle is increased. 


\subsection{Conclusions}

A full-scale tidal turbine was simulated using blade-resolved CFD to analyse the influence of shear velocity profile and blade pitching in power-capping zone on rotor power and thrust, and on wake evolution.

The force coefficients in a shear flow were found to be higher due to the higher velocity flow in the upper portion of the turbine swept area compared to the uniform flow. The loading on the rotor blade during a rotation in a shear flow is azimuthally asymmetric as compared to the symmetric loading in a uniform flow. This unsteady loading in a shear flow will likely reduce the fatigue life of the blades.

The angle of attack along the span of the blade varied from $5^{\circ}-8^{\circ}$ at the design tip speed ratio of 5.5 which produces the optimum lift to drag ratio for the aerofoil sections used in the blade.

The influence of the pitch-to-feather power-capping strategy was examined by studying the forces and angle of attack on the turbine blade, and the wake at three different blade pitch angles. The approach angle on the blade increases as the blade pitch angle is increased due to the decreasing rotor thrust causing an increase in the axial flow velocity. However, due to blade pitching, the angle of attack on the blades decreases even though the approach angle increases, thereby decreasing the power coefficient and maintaining constant power in the power-capping zone.

The decreasing angle of attack on the blade at higher blade pitch angles results in a significant drop in the average load on the blade. The loading distribution on the blade is quite different in all three cases with the point of maximum load along the blade span shifting from the blade tip towards the root with increasing pitch angle.

The decrease in thrust on the rotor has a direct influence on the wake evolution behind the rotor. An increase in blade pitch angle increases the rate of velocity recovery in the 
wake due to lower turbine rotor thrust. This also resulted in a decrease in the wake width and a decrease in the tip vortex strength. Furthermore, the turbulence intensity in the wake decreases with increasing blade pitch angle due to the relatively lower shear between the bypass flow and the flow in the wake.

In this chapter, the support structure was neglected to analyse the influence of other parameters. In the next chapter, two different support structures, a circular cylinder and an elliptical cylinder, will be considered and their influence on the flow will be examined. 


\section{Chapter 6}

\section{Influence of support structures}

\subsection{Introduction}

In the previous chapter, the influence of the shear velocity profile and blade pitching in the power-capping zone was analysed without including a support structure. In this chapter, two different monopile support structures, with circular and elliptical cross-sections, are considered and their influence on the flow is studied.

This chapter starts with the description of the domain and the mesh used for the rotor along with the support structure. The influence of the support structure on the rotor is studied by analysing the forces and the angle of attack distribution along the rotor blades. Later the influence of the rotor on the support structure is discussed by investigating the forces on the support structure. The last section presents the effect of rotor and support structure interaction on the downstream wake. 


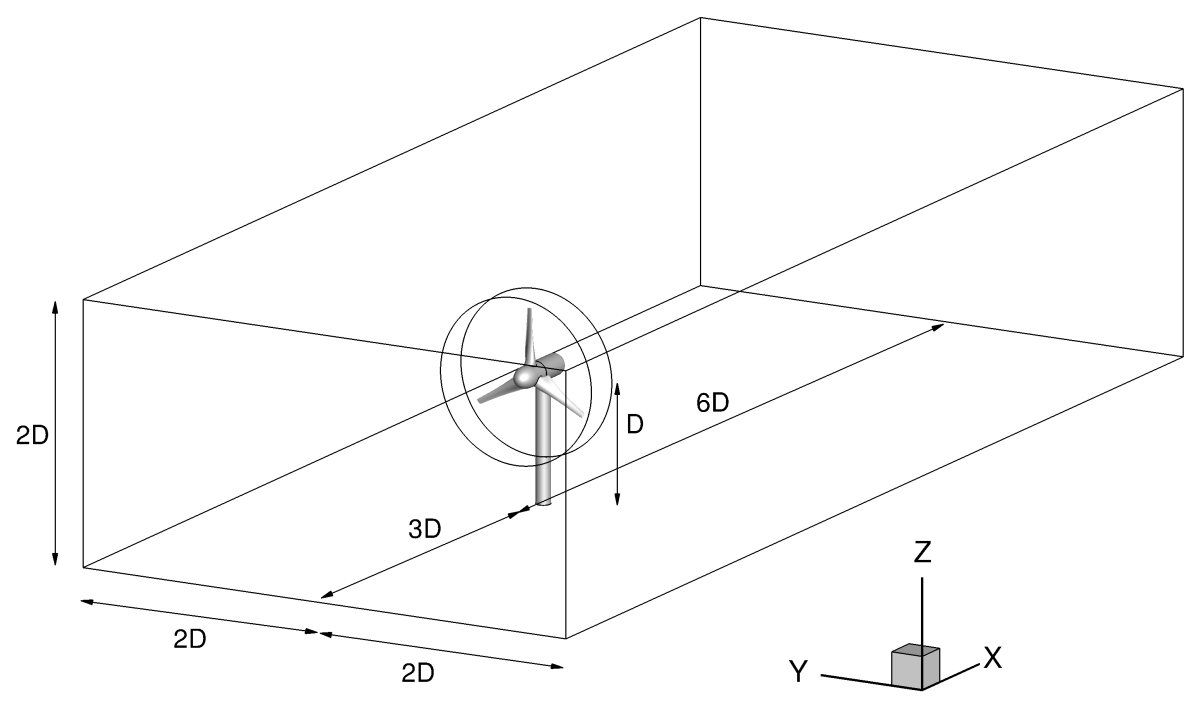

Figure 6.1: Dimensions of the computational domain. The flow direction is in the positive $\mathrm{x}$-direction.

\subsection{Turbine rotor description}

\subsubsection{Computational domain}

The same turbine rotor of $18 \mathrm{~m}$ diameter (D) used in the previous chapter was used for the current simulations. The support structure was $18 \mathrm{~m}$ (D) in height. The cylindrical support structure considered was $2 \mathrm{~m}(\mathrm{D} / 9)$ in diameter and the front of the support structure is located $2.5 \mathrm{~m}$ behind the rotor based on a proprietary design obtained through the PerAWaT project. An elliptical support structure of aspect ratio 2:1 was examined for the other case such that its cross-stream width, the minor axis, is the same as the circular cylinder diameter, $2 \mathrm{~m}(\mathrm{D} / 9)$ and its major axis length in the flow direction is $4 \mathrm{~m}$. The aspect ratio of 2:1 for the ellipse was chosen based on the optimum lift to drag ratio for different aspect ratios of the ellipse as discussed earlier in Chapter 4.

The computational domain has two sub-domains, the inner domain and the outer do- 
main. The inner domain consists of the rotor and a cylindrical coin, 0.33D long and a diameter of 1.25D surrounding it, excluding the support structure; see figure 6.1. The outer domain contains part of the nacelle, the support structure and the remaining computational domain. To simulate turbine rotation, the mesh in the inner domain is physically rotated whereas the outer domain remains stationary and the data transfer occurs through the interface between the two domains. The details of data transfer through the interface were discussed in Chapter 4. Hence an unsteady simulation is carried out to capture the unsteady nature of the rotor - support structure interaction.

\subsubsection{Mesh}

The same meshing strategy used for the inner domain in the previous chapter was used in this simulation. A C-grid was used around the blade with sections defined along the span of the blade to have better control over the span-wise distribution of mesh elements. The outer domain has part of the nacelle and the support structure and hence a new strategy was adopted for meshing the outer domain. An O-grid was used around the support structure to capture the boundary layer and the first cell wall height on the support structure was chosen such that the wall $\mathrm{y}^{+}$is less than 5 , thus fully resolving the boundary layer; see figure 6.2. The mesh distribution in the $\mathrm{O}$ - grid was based on the validation studies discussed in Chapter 4 and the total number of nodes around the circumference of the $\mathrm{O}$ - grid was set to 120 . The span-wise mesh distribution on the support structure was such that the portion of the support structure which would interact with the tip vortices from the blade was resolved finer than the rest of the structure. The rotor blade-tip at its bottom most point is at half the support structure length (L) and hence the span-wise mesh on the support structure around this region is finer than the rest of the support structure. Note that as the wake expands, the portion of the support structure that interacts with it is lower than 
$\mathrm{L} / 2$ which is taken into consideration for the finer mesh.

The overall mesh count in the inner domain is 4.77 million which is the same as in the previous chapter without support structure. In the outer domain, the overall mesh count is about 2.1 million compared to 1.05 million in the previous chapter due to the presence of the support structure and much finer mesh around it.

Table 6.1: Mesh cell count

\begin{tabular}{|c|c|}
\hline Region & Cell count \\
\hline Inner domain & 4.77 million \\
\hline Outer domain & 2.1 million \\
\hline Blade chord & 60 \\
\hline Blade span & 50 \\
\hline Support structure circumference & 120 \\
\hline Support structure span & 52 \\
\hline
\end{tabular}

\subsubsection{Boundary conditions}

The domain inlet and outlet are set as velocity inlet and pressure outlet respectively. Both the side boundaries, and the top boundary are set as symmetry planes. The inlet boundary conditions are specified similarly as in the previous chapter based on the shear flow model of Fleming et al. (2013), such that the depth-averaged inlet velocity is $2 \mathrm{~ms}^{-1}$. To sustain 


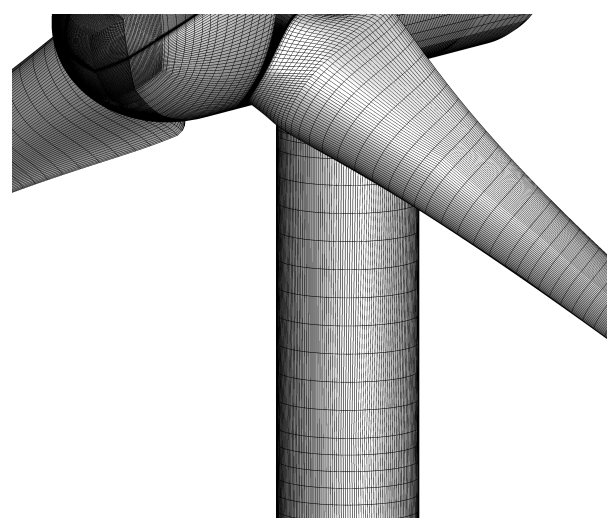

(a) Circular cylinder mesh

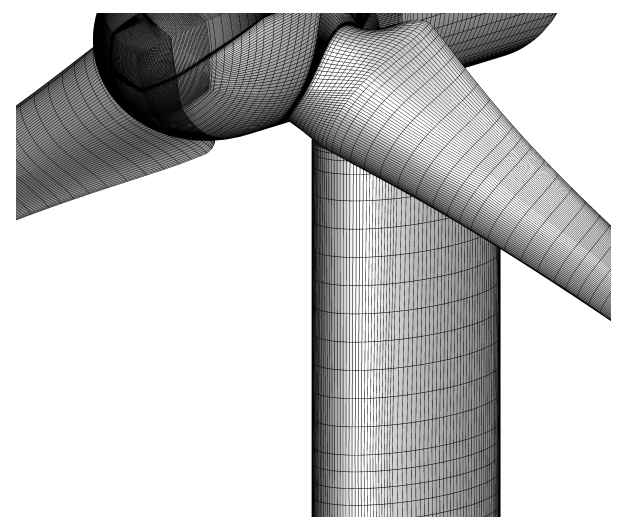

(b) Elliptical cylinder mesh

Figure 6.2: Structural mesh around the blade and the support structure. $\mathrm{O}$ - grid was used around the support structure in the outer domain.

the shear profile in the flow velocity along the length of the domain, a wall shear stress is applied at the bottom of the domain (Fleming et al., 2013). The shear in the velocity profile changes the velocity of approach along the span of the support structure, the effect of which is discussed later in this chapter. The inlet turbulence intensity was specified based on the Fleming et al. (2013) model as discussed in the previous chapter. The turbulence intensity at the inlet varies from $0 \%$ at the free surface to about $12 \%$ at the channel bottom.

The blade surface was defined as a wall boundary and wall functions were used to account for the boundary layer as discussed in the previous chapter. The support structure was specified as a wall boundary condition with the velocity and turbulent kinetic energy being zero at the wall. Since the mesh is fully resolved, the wall functions are not needed.

\subsection{Flow physics in the presence of support structure}

\subsubsection{Power and thrust coefficients of the rotor}

The turbine rotor is simulated in the presence of two different support structures, ellipse and cylinder. To study the influence of the support structures over the tidal velocity range, 
each support structure case is simulated at three different points, at the turbine rated speed, near the rotor cut-out speed and one point in between. The turbine rated speed corresponds to the $0^{\circ}$ blade pitch angle whereas the cut-out speed corresponds to the $20^{\circ}$ blade pitch angle. The third point in-between these is simulated at $10^{\circ}$ blade pitch angle. The results from these three data points will be used in the reassessment of the analytical channel model, discussed earlier in Chapter 2, which will be discussed in the next chapter. The results of the $10^{\circ}$ blade pitch angle are presented in this chapter as it is might be a good representative case, being the median of the range of pitch angles considered. The flow features in the presence of support structures for the $10^{\circ}$ blade pitch angle are compared to the simulation without any support structure from the previous chapter.

Table 6.2: Time-averaged rotor force coefficients in the presence of different support structures at $10^{\circ}$ blade pitch angle

\begin{tabular}{|c|c|c|c|}
\hline Support type & TSR & $\overline{C_{P}}$ & $\overline{C_{T}}$ \\
\hline No Support & 4.3 & 0.308 & 0.372 \\
\hline Circular cylinder & 4.3 & 0.325 & 0.392 \\
\hline Elliptical cylinder & 4.3 & 0.315 & 0.385 \\
\hline
\end{tabular}

The presence of support structures increases the blockage in the domain which results in higher opposing thrust in the domain causing an increase in the local flow velocity near the rotor; see figure 6.3. Below the nacelle, the presence of support structure can be clearly observed with the cylinder case having the lowest axial flow velocity through the rotor followed by ellipse case. Above the nacelle, the local flow velocity through the rotor is slightly higher for the cylinder case followed by ellipse case compared to the no support 


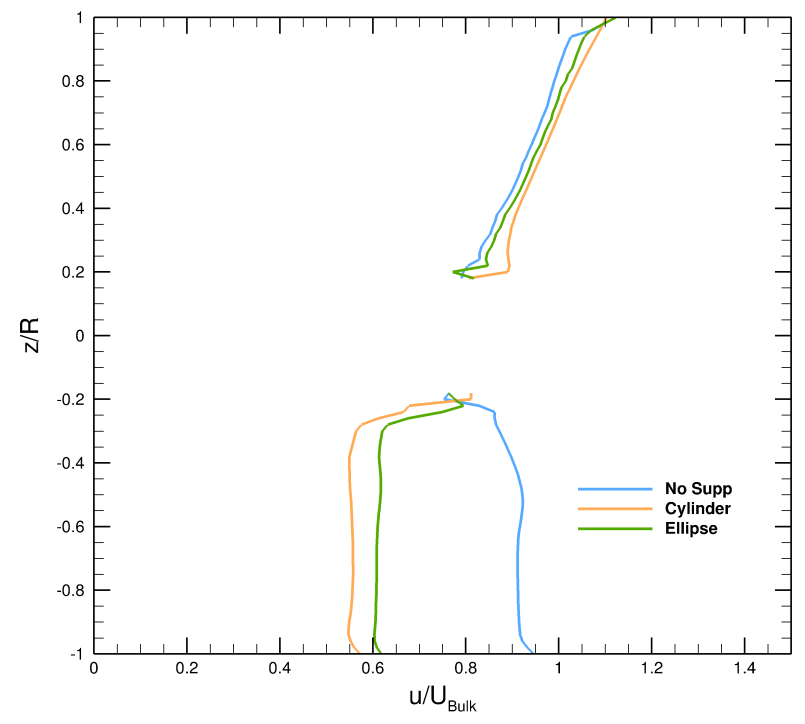

Figure 6.3: Vertical profile of mean axial velocity through the rotor normalized with the depth-averaged bulk flow velocity.

structure case due to the higher blockage effect of the cylinder compared to the ellipse.

As the rotor blades are away from the support structure most of the time during a rotation, they experience the slightly higher local flow velocity causing an increase in the rotor power and thrust coefficients. In the case of a cylindrical support structure, the timeaveraged rotor power and thrust coefficients increased by 5.5\% and 5.3\% respectively whereas in the case of an elliptical support structure, the same coefficients increased by $2.3 \%$ and $3.4 \%$ compared to the case without any support structure; see table 6.2 . The higher drag coefficient of the circular cylinder causes higher opposing thrust to the flow increasing the local flow velocity and hence a larger increase in the power and thrust coefficients compared to an ellipse with a lower drag coefficient. Since the simulation is in a constrained domain with constant inflow velocity, there is an increase in the rotor power and thrust coefficients but in the case of a head-driven channel flow, the increased opposing thrust might have a feedback effect on the inflow velocity which will be discussed using the analytical channel model in the next chapter. 


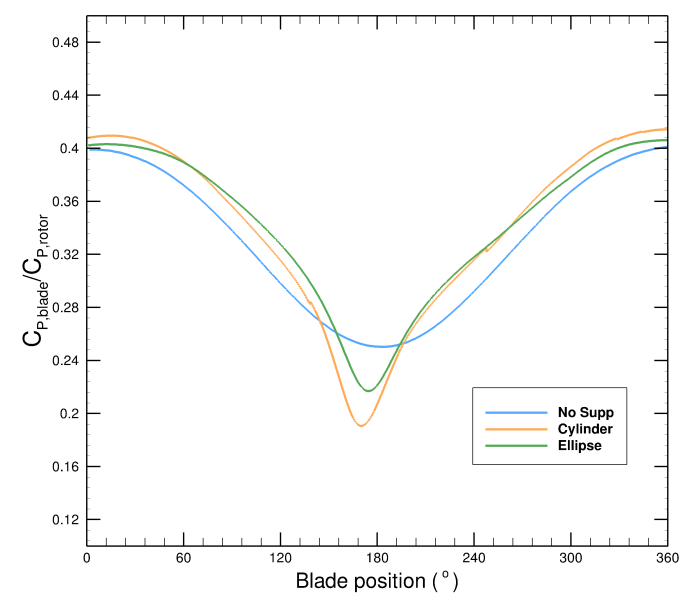

(a) Normalised power coefficient

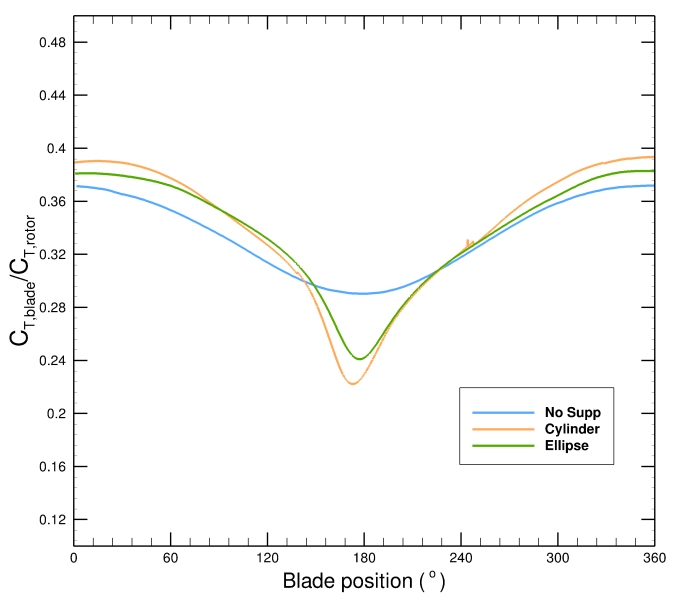

(b) Normalised thrust coefficient

Figure 6.4: Blade power (a) and thrust (b) coefficients normalised with respect to the rotor force coefficients for different support structures, cylinder and ellipse, at $10^{\circ}$ blade pitch angle during one rotation cycle.

The force on a turbine blade during a rotation is not constant due to the shear in the velocity profile and also due to the presence of a support structure. The blade force coefficients normalised by the rotor force coefficients for different support structures at $10^{\circ}$ blade pitch angle are presented in figure 6.4. In the case of cylindrical support, the maximum reductions from the uniform value, discussed in the previous chapter of about 0.33 , in normalised blade power and thrust coefficients are 39\% and 30\% respectively whereas in the case of an elliptical support, these reductions are $30 \%$ and $25 \%$ respectively which are higher than in the shear flow case with no support structure; see figure 6.4. This is due to the higher velocity flow in the presence of support structures in the upper portion of the domain and lower velocity region in the lower portion of the domain near the rotor plane as discussed earlier. The higher blockage of cylinder causes a larger variation in the power and thrust coefficients compared to the elliptical support structure.

The minimum in the power coefficient occurs at $166^{\circ}$ and $170^{\circ}$ in the case of cylinder and ellipse respectively whereas the corresponding minimum in the thrust coefficient oc- 
curs at $170^{\circ}$ and $175^{\circ}$. In the absence of a support structure, the minimum occurs at the bottom blade position of $180^{\circ}$. The presence of the support structure changes the angle of attack on the blade as it approaches the support structure thus causing a shift in the minimum, which is discussed in the next section. The cylinder with higher drag has a higher influence on the angle of attack on the blade due to its larger impact on axial flow velocity through the rotor as discussed earlier, and hence results in a larger shift in the azimuthal position of the minimum value.

\subsubsection{Sectional blade forces}

The asymmetry in the blade forces is investigated in detail in this section by studying the angle of attack and sectional forces on the blade during a rotation cycle.

The angle of attack, $\alpha(=\phi-\beta), \phi$ being the angle of approach and $\beta$ being the blade pitch angle, on the blade for different support structures is presented in figure 6.5. The vertical asymmetry is due to the shear in the velocity profile and also the presence of support structure. A lateral asymmetry was also observed due to the blade approaching and leaving the support structure, as observed in the previous section with integrated force on a single blade. In comparison to the case without the support structure, the angle of attack drops near the lowest point in the case of an elliptical support and this drop is even higher in the presence of a cylindrical support as the higher drag of the cylindrical support causes a higher opposing thrust to the flow, hence decreasing the stream-wise velocity and also the angle of attack.

The variation in the angle of attack at the top and bottom position for the blade is presented in figure 6.6. The angle of attack on the blade at the top position is slightly higher for the cylinder case followed by the ellipse case and the no support case. This is due to the higher axial velocity through the rotor plane in the cylinder case as discussed 


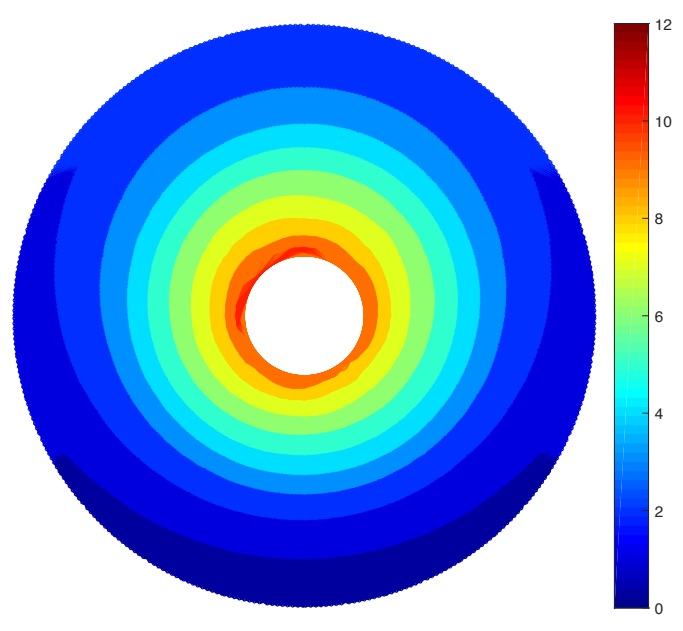

(a) No support

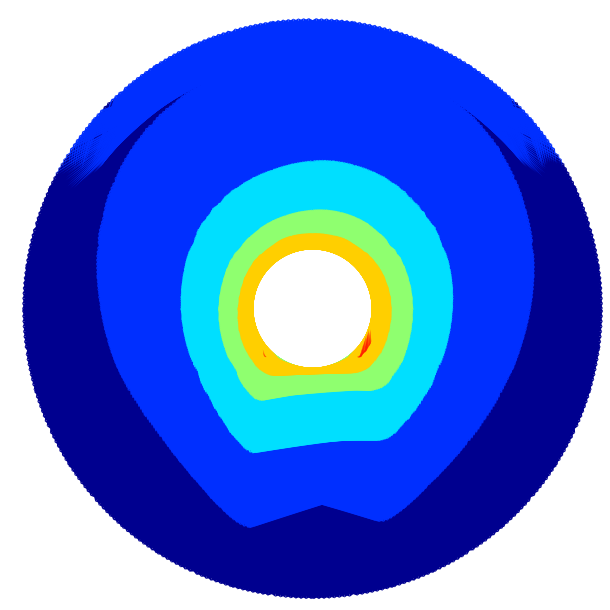

(b) Cylindrical support

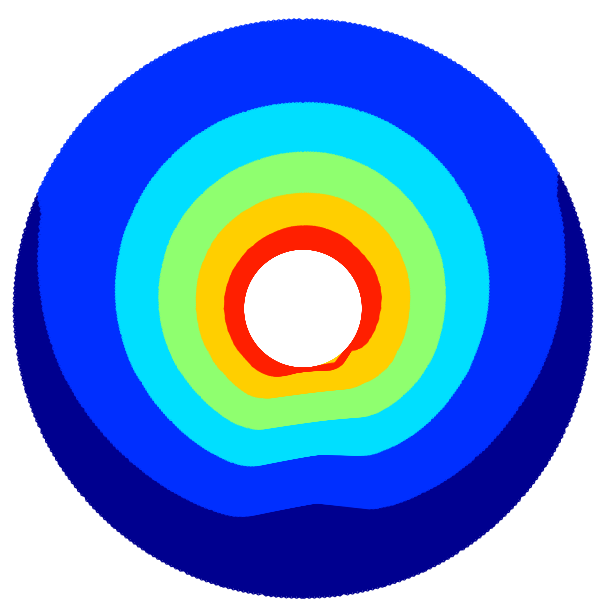

(c) Elliptical support

Figure 6.5: Spanwise variation of angle of attack, $\alpha^{\circ}$, on a turbine blade for different support structures at $10^{\circ}$ blade pitch angle during a rotation cycle. 


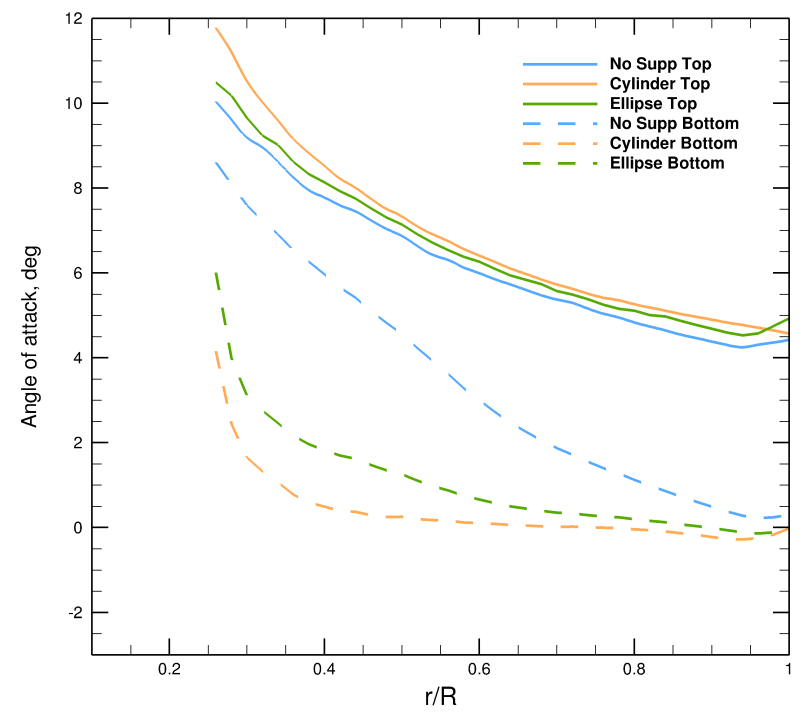

Figure 6.6: Angle of attack variation along the span of a turbine blade in the presence of different support structures for $10^{\circ}$ blade pitch angle, at the top (solid line) and bottom (dashed line) position in a rotation cycle.

in the previous section. Similarly the lower axial velocity at the bottom position causes a larger drop in the angle of attack in the cylinder case followed by the ellipse case and a more gradual drop in the case of no support structure.

The axial sectional force variation on a blade in the presence of different support structures for $10^{\circ}$ blade pitch angle is presented in figure 6.7. The axial force is higher in the top half of the cycle than the lower half due to the higher shear velocity flow in the top half. The presence of the support structure influences the blade forces around the lowest point in the rotational cycle.

The maximum and minimum axial force along the blade span during the rotation is affected by the presence of support structures. The minimum axial force on the blade near the lowest point drops by $21 \%$ in the case of elliptical support and by $29 \%$ in the case of cylindrical support compared to the case without any support structure; see figure $6.8 \mathrm{~b}$. This is due to the change in pressure distribution around the blade in the presence 


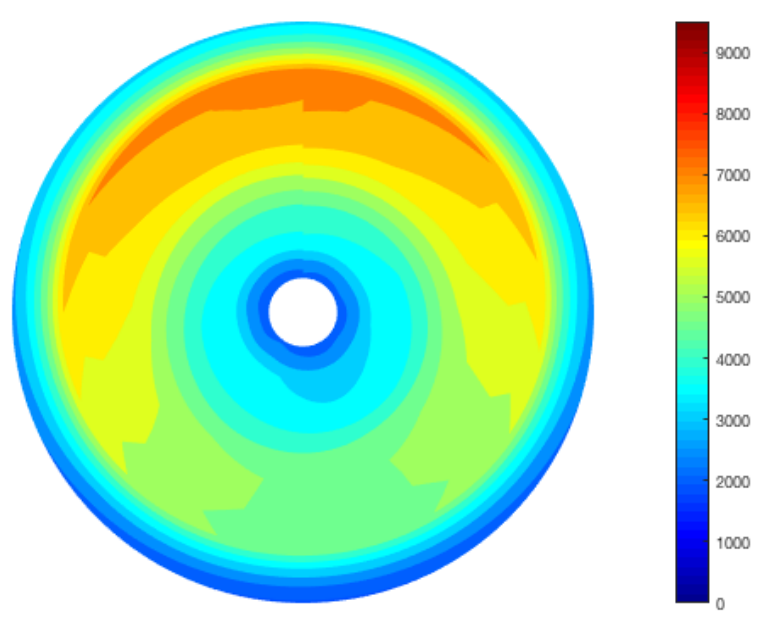

(a) No support

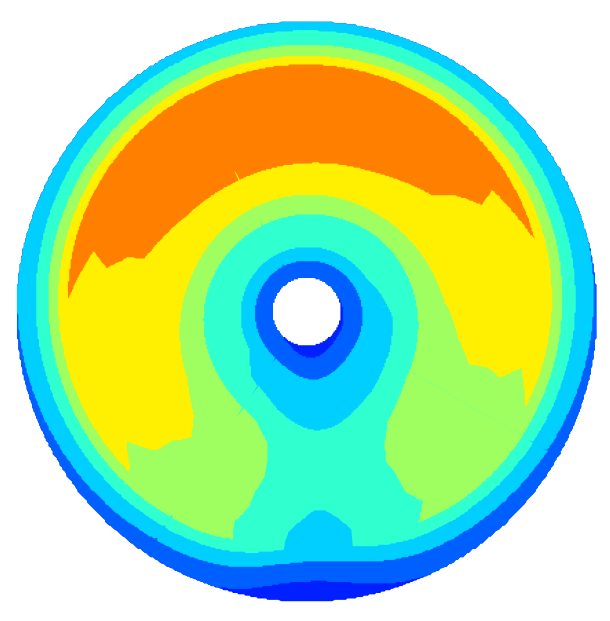

(b) Cylindrical support

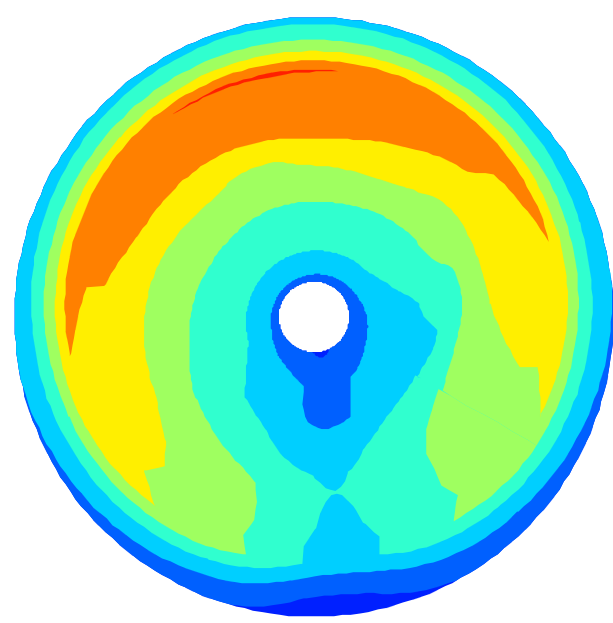

(c) Elliptical support

Figure 6.7: Spanwise axial force $\left(\mathrm{N} \mathrm{m}^{-1}\right)$ variation on a blade for different support structures, cylinder and ellipse, in shear flow. 


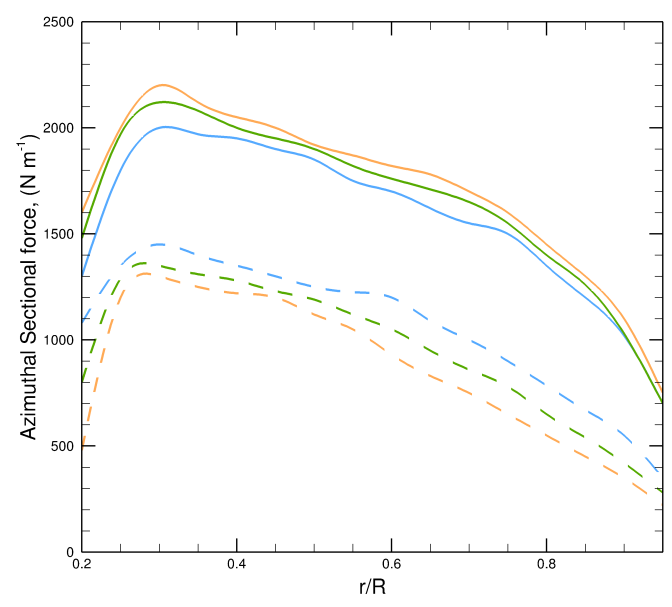

(a) Azimuthal force

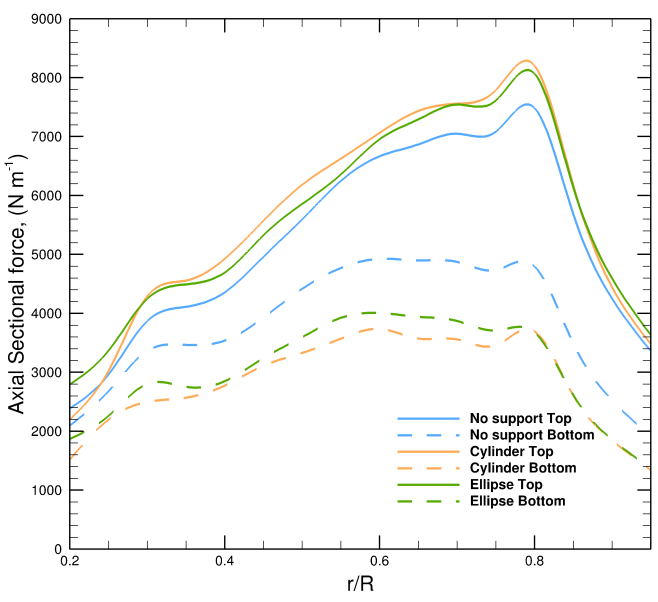

(b) Axial force

Figure 6.8: Sectional force variation along the span of a turbine blade for different support structures, cylinder and ellipse, at the top and bottom position in a rotation cycle.

of a support structure and is in accordance with the decrease in angle of attack observed earlier.

The increase in local blockage caused by the support structure increases the local flow velocity around the top half of the rotor causing an increase in the maximum axial sectional forces along the span of the blade. The maximum axial sectional force along the blade span increases by $8 \%$ in the case of elliptical support and by $11 \%$ in the case of cylindrical support compared to the case without any support structure.

The azimuthal force variation on the blade at its top and bottom position for different support structure cases is shown in figure 6.8a. In the presence of a cylindrical support, the maximum azimuthal sectional force increases by $12 \%$ compared to the case without support structure whereas this increase is $7 \%$ in the case of an elliptical support.

As the blade spends most of the time during its rotation away from the support structure, this increase in forces on the blade causes an overall increase in the integrated power and thrust coefficients of the rotor, as observed in the previous section. 
Table 6.3: Values of the mean stream-wise force coefficients of different support structures behind the rotor at $10^{\circ}$ blade pitch angle

\begin{tabular}{|c|c|c|}
\hline Support type & TSR & Stream-wise force coefficient $\left(C_{D}\right)$ \\
\hline Cylinder & 4.3 & 0.21 \\
\hline Ellipse & 4.3 & 0.12 \\
\hline
\end{tabular}

\subsection{Forces on support structure}

The mean stream-wise force coefficients on the support structures averaged over their height are presented in table 6.3. The mean stream-wise force coefficients on the support structures in the presence of the rotor are considerably lower than for the support structures by themselves in open-water, considered during the validation studies in chapter 4 . In the presence of the rotor, the drag coefficient of the cylinder drops by $64 \%$ and that of the ellipse drops by $30 \%$. Note that the drag coefficient in this case is based on the depthaveraged inlet velocity which might not be the true flow velocity incident on the tower since the flow velocity reduces behind the rotor and the velocity of the approach flow onto the support structure is lower than the inlet velocity. Also the stream-wise and crossstream forces on the support structure are not similar to the lift and drag forces on a bluff body in open water because the swirl induced from the rotor changes the angle of attack on the support structure. Hence both the stream-wise and cross-stream forces include components of lift and drag forces. This can be noticed from the stagnation line on the support structure which in open water in the absence of the rotor would be at the centre all along its length but due to the swirl behind the rotor, it shifts towards one end; see figure 6.9. This indicates that a non-zero mean cross-stream force would act on the support structure (discussed in the next section). This force is much higher in the case of an ellipse compared to the circular cylinder because the angle of attack plays a more significant role 


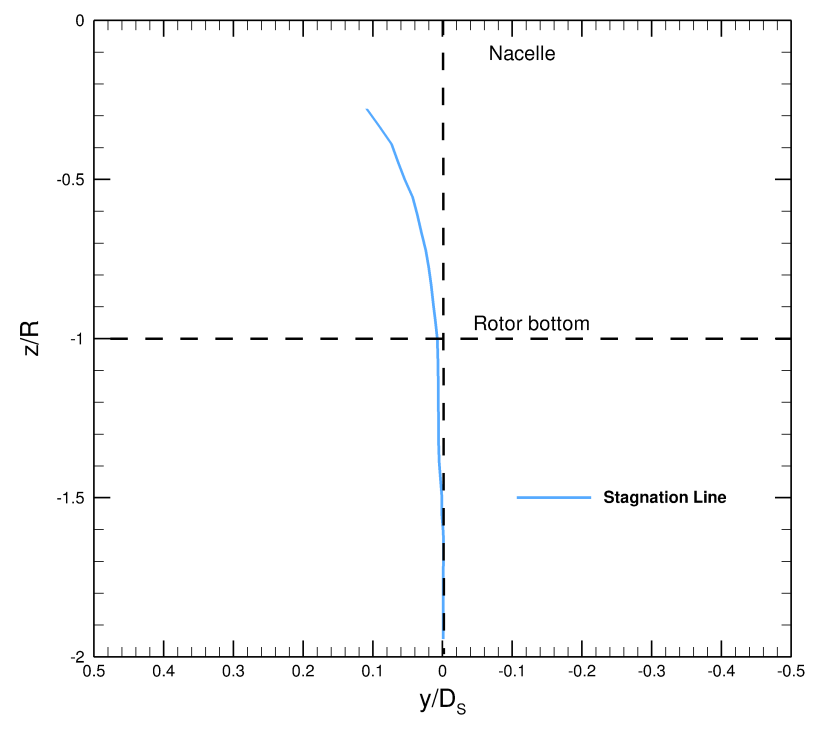

Figure 6.9: Stagnation line on the frontal area of the cylindrical support structure. $\mathrm{D}_{S}$ represents the diameter of the cylinder and y represents the variation along the diameter in the direction transverse to the flow. The dashed line indicates the stagnation line in the absence of a rotor. The deviation can be observed behind the rotor.

in the case of an ellipse than a cylinder as will be discussed in the next section.

\subsubsection{Support structure sectional force variation}

The stream-wise sectional forces on the support structure at three different vertical heights, two behind the rotor swept area $(\mathrm{z}=-0.4 \mathrm{R}$ and $\mathrm{z}=-0.8 \mathrm{R})$ and one below the rotor $(\mathrm{z}=$ $-1.2 \mathrm{R}$ ), are presented in figure 6.10 . The presence of the rotor causes a dip in the sectional forces on the support structures, both cylinder and ellipse. These sectional forces are periodic with a frequency equal to the blade passing frequency.

Stream-wise forces on sections directly behind the rotor have a much higher amplitude of fluctuation due to the influence of the passing blade. As the blade approaches the support structure, there is a decrease in velocity of approach to these sections and a consequent decrease in the stream-wise force. 


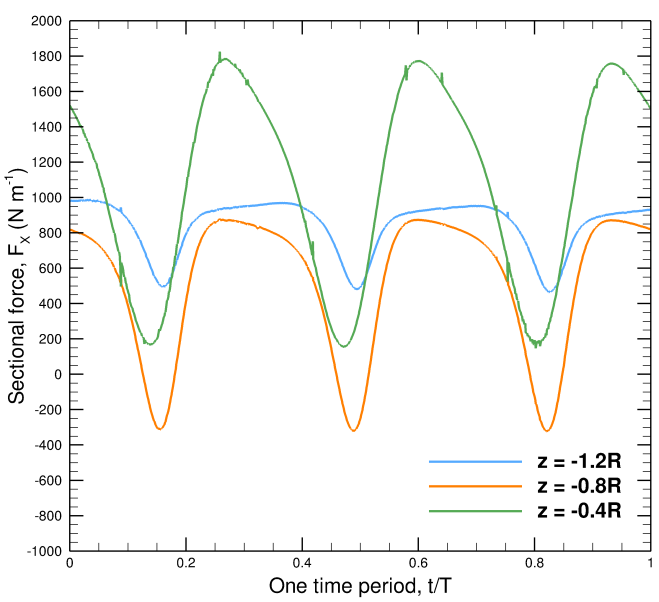

(a) Cylindrical support

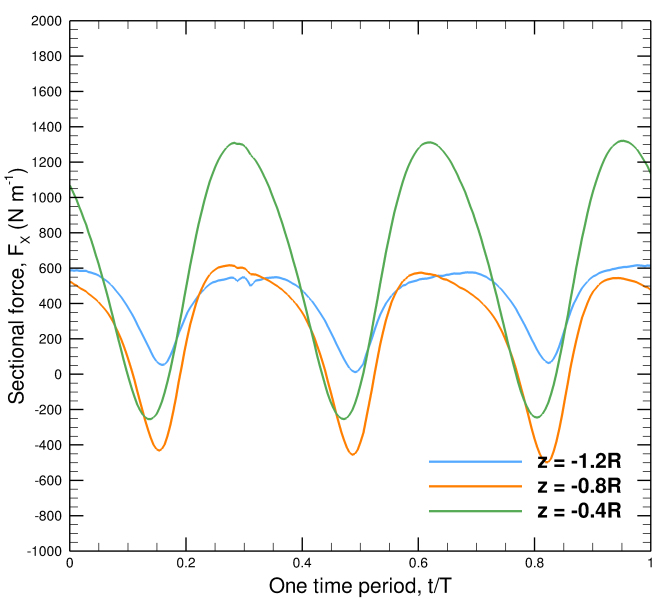

(b) Elliptical support

Figure 6.10: Stream-wise sectional forces on support structures, cylinder (a) and ellipse (b) during one rotation cycle.

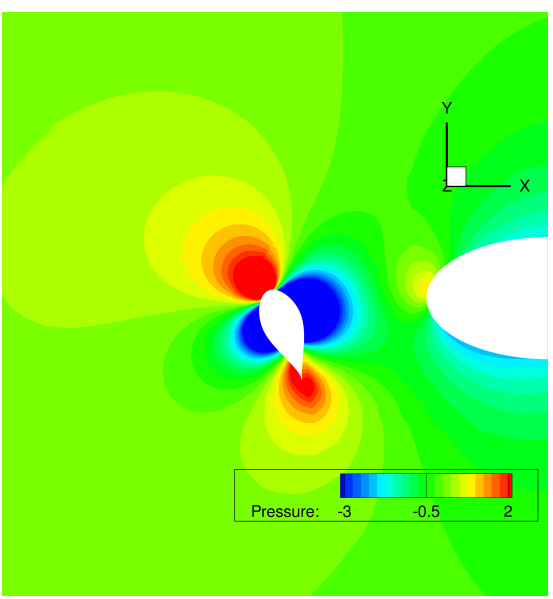

(a) $\mathrm{z} / \mathrm{R}=-0.4$

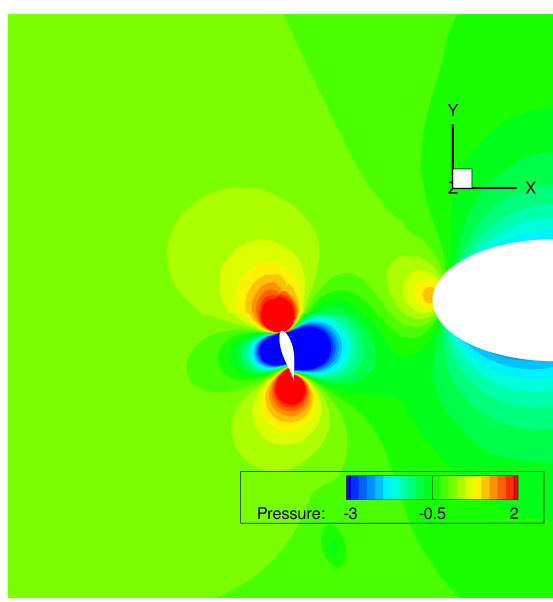

(b) $\mathrm{z} / \mathrm{R}=-0.8$

Figure 6.11: Pressure slices at $t / T=0.15$. The leading edge of the blade section at $\mathrm{z} / \mathrm{R}=$ -0.4 (a) has just passed the centre line of the support structure while the blade section at $\mathrm{z} / \mathrm{R}=-0.8$ (b) is just approaching the centre line. 


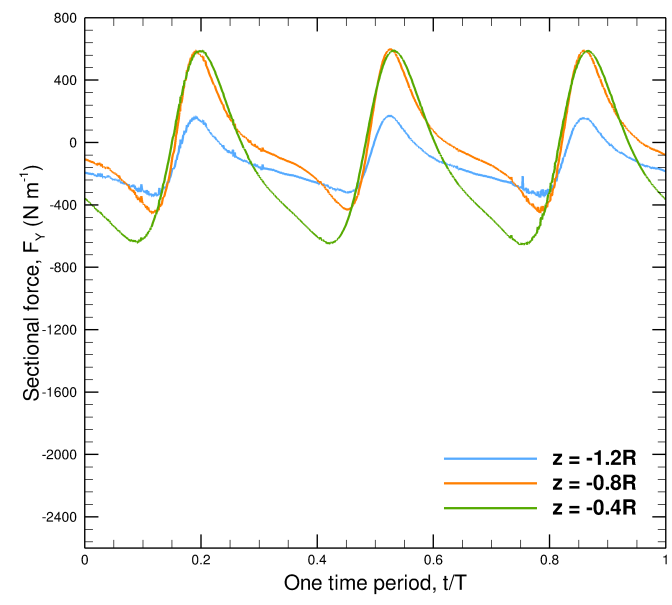

(a) Cylindrical support

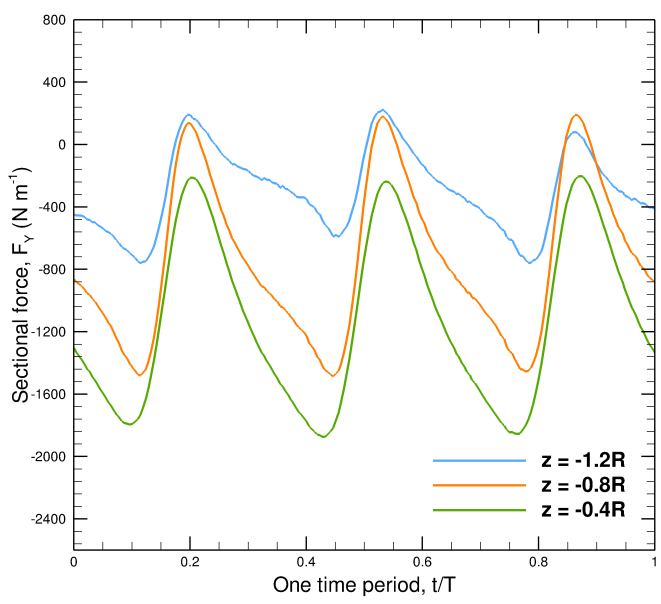

(b) Elliptical support

Figure 6.12: Cross-stream sectional forces on support structures, circular (a) and ellipse (b) during one rotation cycle.

The minimum force at different vertical sections occurs at slightly different points in time because the support structure section closer to the blade root will experience a change in pressure earlier than the section at the blade tip due to the thrust from the rotor blade affecting the flow. In figure 6.11 , the pressure distributions at blade sections, $\mathrm{z} / \mathrm{R}=-0.4$ and -0.8 , are shown at the same time instant. In figure $6.11 \mathrm{a}$, the leading edge of the blade section has just passed the centre line of the support structure while in figure $6.11 \mathrm{~b}$, leading edge of the blade section is just approaching the centre line. The consequent influence near the stagnation point on the support structure can be clearly noticed with different pressure at different sections. Hence the minimum value for $\mathrm{z}=-0.4 \mathrm{R}$ section occurs earlier than the other sections. This might be an important factor in the design of the support structure because the minimum force along the length of the support structure does not occur at the same instant. In the case of the elliptical support, the stream-wise sectional forces are lower than that of the cylindrical support due to the lower drag coefficient of the ellipse. Also notice that the stagnation point on the support structure section is off the centre line 
as discussed earlier.

The cross-stream sectional forces on the support structure are presented in figure 6.12. As in the case of stream-wise forces, the cross-stream forces at the sections directly behind the rotor have a much higher amplitude of fluctuation. The presence of the rotor also causes an asymmetry in the forces as compared to a support structure by itself in openwater. In the case of an ellipse, there is a large mean force in the cross-stream direction on the support structure which is discussed in the next section.

\subsubsection{Mean sectional force along length of the support structure}

The mean forces on the support structure along its length are presented in figure 6.13. There is a drop in the forces at heights, $0<\mathrm{L} / \mathrm{D}<0.5$, corresponding to the location of the rotor. The presence of the rotor reduces the velocity behind it and hence lower axial forces, $\mathrm{F}_{x}$ on the support structure.

The mean cross-stream forces on the support structure are negative indicating that the direction of the force is opposite to the blade rotation; see figure 6.11. This is in accordance with the location of the stagnation line, figure 6.9 , which indicated that a non-zero mean force would act in the -Y direction. In the case of an ellipse, this force is about 50\% higher which should be considered during the design of the support structure.

Overall the mean axial force on the cylindrical support structure, at heights corresponding to rotor location, is about $70 \%$ lower than in the case of a support structure by itself in open-water and hence the dimensions of a cylindrical support structure might be reduced considering a reduction in forces. In the case of an elliptical support, the higher crossstream forces may be the dominating factor in deciding the dimensions of an elliptical support structure. 


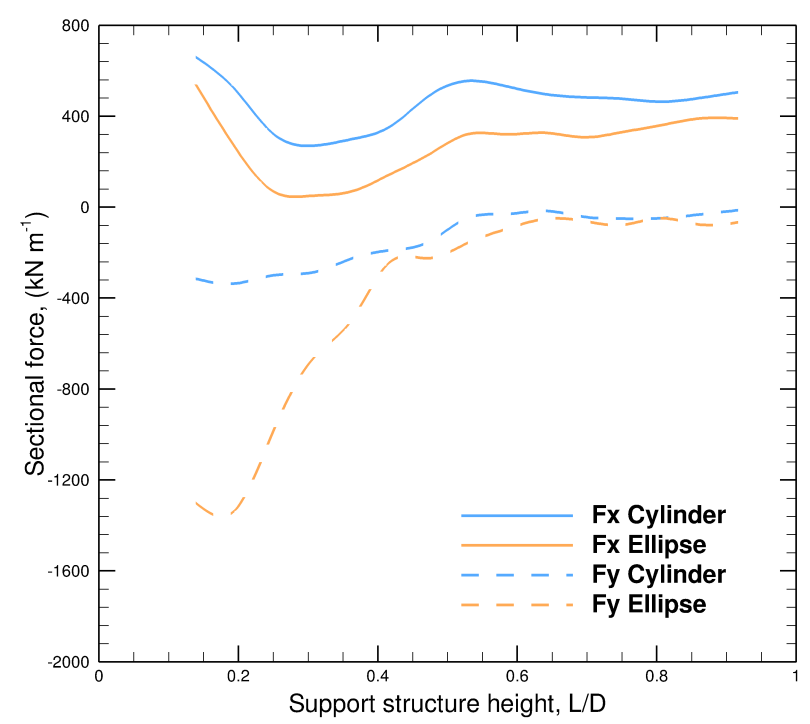

Figure 6.13: Mean sectional force variation along the support structure height, $\mathrm{L} / \mathrm{D}=0$ at the hub and $\mathrm{L} / \mathrm{D}=1$ at the seabed. $\mathrm{F}_{x}$ is in the direction of the flow and $\mathrm{F}_{y}$ being in the cross-stream direction.

\subsection{Wake comparison}

In the previous sections, the variation in rotor and support structure thrust for different support structures was studied. This thrust has a direct influence on the flow velocity in the wake which will be discussed in this section.

The velocity deficit at different stream-wise locations in the wake is presented in figure 6.14. The plot at $0.125 \mathrm{D}$ is the location just ahead of the support structure. At this location, the velocity deficit is much higher in the presence of support structures due to the stagnation region ahead of the support structure. The plot at $\mathrm{x} / \mathrm{D}=0.125$, behind the lower half of the rotor, $0>\mathrm{z} / \mathrm{D}>-0.5$, the rotor thrust further decreases the velocity in the wake which can be observed as an increase in the velocity deficit. In the presence of a cylindrical support, the velocity deficit is about $20 \%$ higher than in the case of an ellipse due to the higher opposing thrust from the cylinder.

At locations further downstream, the influence of support structure can be clearly ob- 

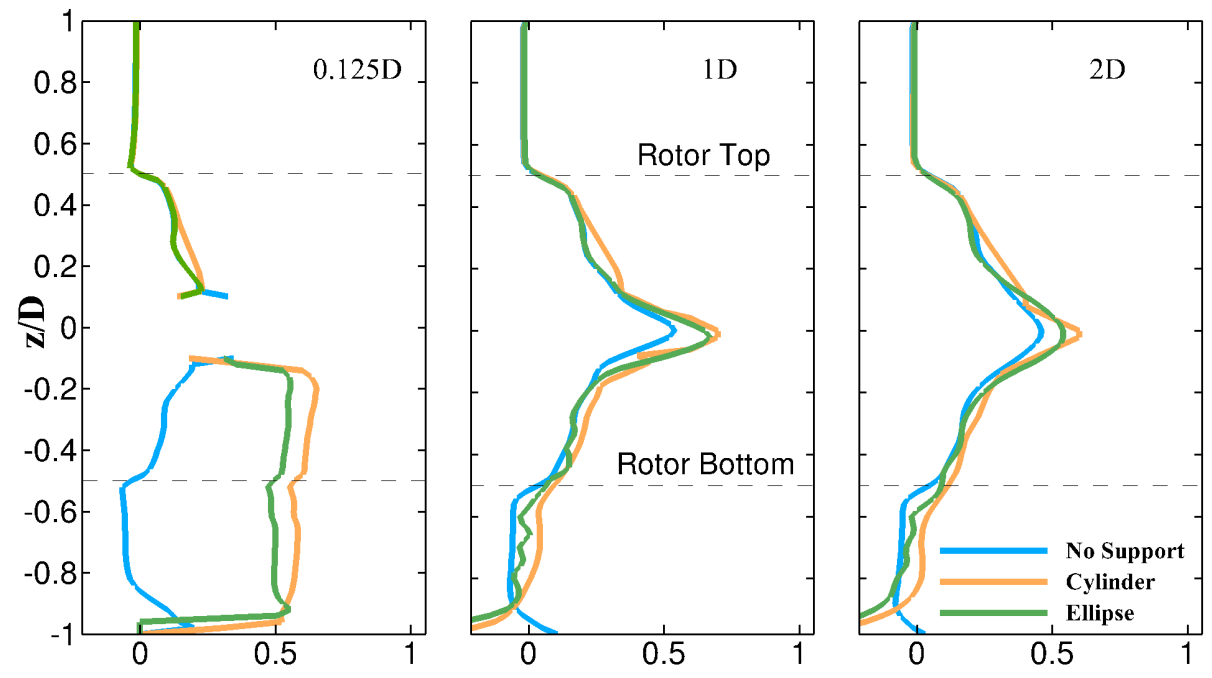

Velocity deficit, 1 - u/ $\mathbf{U}_{\mathbf{x}}(\mathrm{z})$

Figure 6.14: Vertical variation in normalised velocity deficit at three different streamwise locations, $x / D=0.125,1$ and 2 in the near wake for the $10^{\circ}$ blade pitch angle. The velocities are normalised with respect to the inlet shear profile. $0.125 \mathrm{D}$ is the location just ahead of the support structure.

served causing a higher velocity deficit. The cylindrical support structure causes a higher velocity deficit followed by the elliptical support and then the case with no support structure, corresponding to their drag coefficients. At sections $x / D=1$ and 2 in the wake, in the absence of support structures, the velocity at $-0.5<\mathrm{z} / \mathrm{D}<-1$ is higher than the corresponding inlet flow velocity due to the bypass flow around the turbine rotor, thus resulting in a negative velocity deficit. A similar effect is observed in the presence of an elliptical support but in the presence of a cylindrical support, its higher drag causes a reduction in the bypass flow velocity, resulting in an overall positive velocity deficit; see figure 6.14. Also the velocity deficit is slightly higher for the cylinder case above the nacelle, $0<\mathrm{z} / \mathrm{D}$ $<0.5$, due to the higher rotor thrust in the cylinder case as discussed in Section 6.3.1. The contours of higher velocity deficits behind the support structures can be observed in figure 6.15 . 


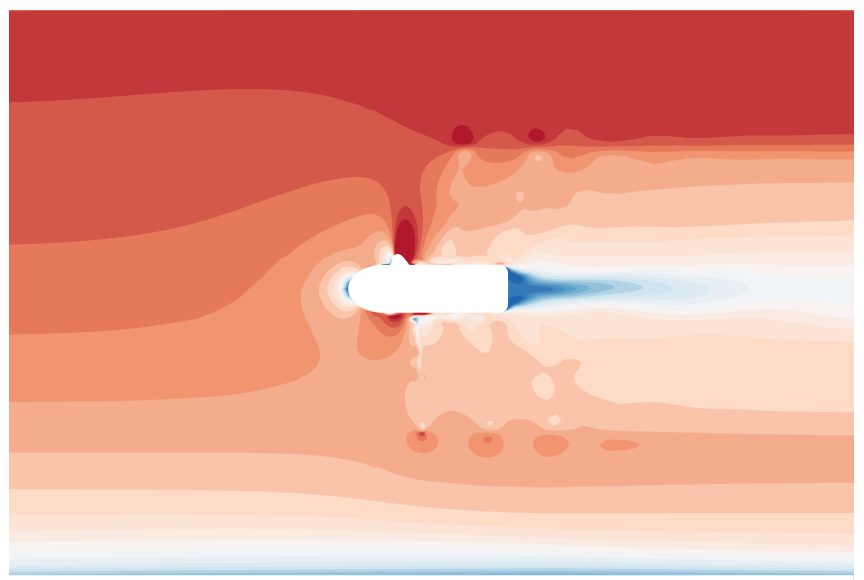

(a) No support

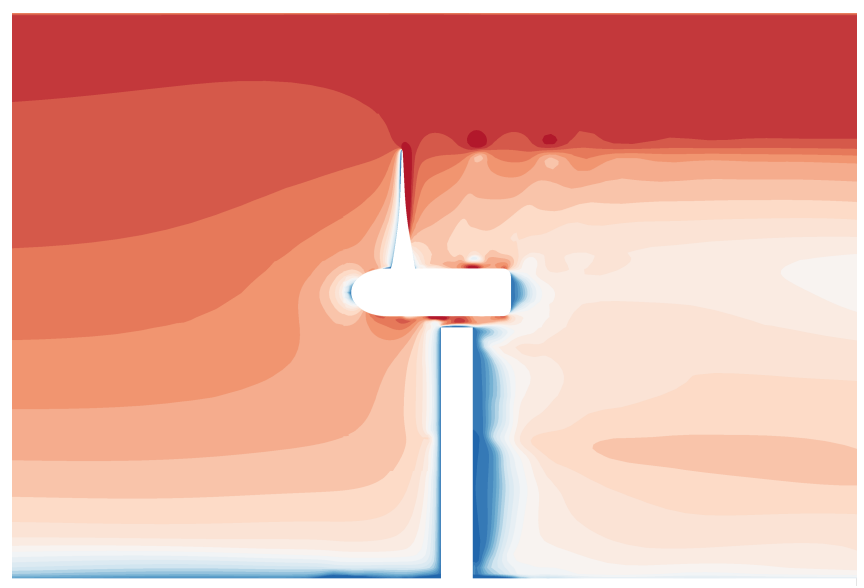

(b) Cylindrical support

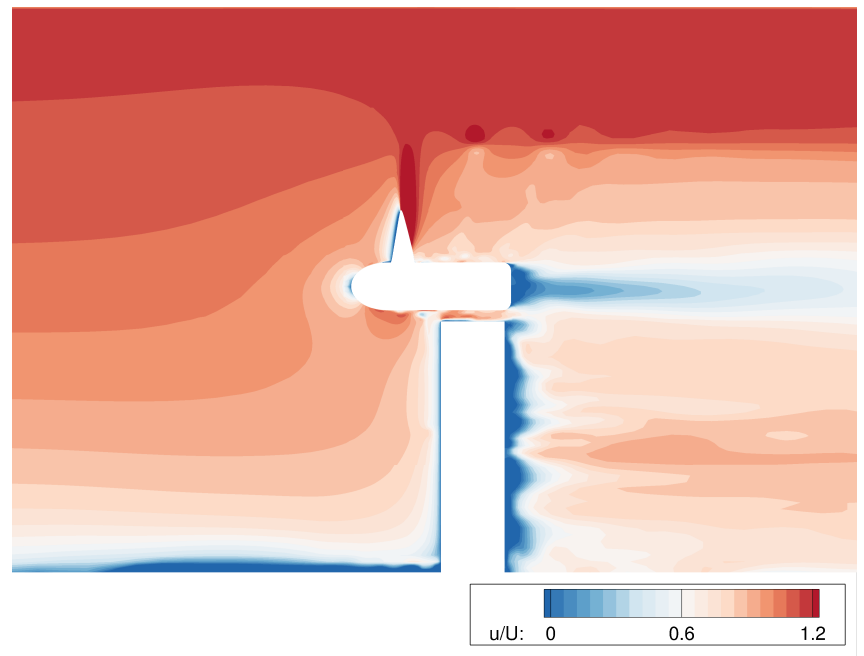

(c) Elliptical support

Figure 6.15: Variation in normalised axial velocity at lateral mid-section for different support structures, cylinder and ellipse. The velocities are normalised with depth-averaged velocity. 


\subsection{Conclusions}

A full-scale tidal turbine was simulated in the presence of two different support structures, cylinder and ellipse, to study the flow interaction between the rotor and the support structure.

The integrated rotor force coefficients were higher in the presence of the cylindrical support structure than the elliptical support due to the higher opposing thrust from the cylinder in the channel increasing the local flow velocity near the top half of the rotor.

The variation in the force coefficients over a single blade rotation was also higher in the presence of support structures because they influence the pressure distribution around the blade when it passes in-front of the support structure. As a result, the minimum in the force coefficient does not occur at exact bottom, $180^{\circ}$. The minimum in the power coefficient occurs at $166^{\circ}$ and $170^{\circ}$ for cylinder and ellipse respectively whereas the minimum in the thrust coefficient occurs at $170^{\circ}$ and $175^{\circ}$.

The angle of attack on the blade decreases as it approaches the support structure due to the reduction in stream-wise flow velocity just ahead of the support structure. This drop is higher for cylindrical support than the elliptical support due to its larger opposing thrust to the flow causing a larger drop in stream-wise velocity. The drop in the angle of attack due to the presence of support structures causes a significant drop in the blade sectional forces as it approaches the support structure.

The presence of rotor also causes a drop in the forces on the support structure. The mean stream-wise force coefficients of the cylindrical and elliptical support structures are 0.21 and 0.12 respectively, which are much lower than if they were in open water without a rotor. The presence of rotor reduces the approach flow velocity on the support structures which reduces the stream-wise force on them. The amplitude of the stream-wise sectional forces was lower in the case of an ellipse than the cylinder due to the lower drag coefficient 
of the ellipse.

The swirl from the rotor induces a mean cross-stream force component on the support structure which is much higher in the case of an ellipse than the cylinder. This is because a small change in the angle of attack causes a large difference in the forces on the ellipse than on the cylinder as seen in Chapter 4. This might be the driving factor in designing an elliptical support.

For both the support structures, the stream-wise sectional forces behind the rotor swept area are lower than in open water whereas the mean cross-stream forces are higher. This should be considered while designing the dimensions of the support structures.

The thrust from the rotor and the support structure has a direct influence on the wake velocity. The velocity deficit immediately upstream of the support structure is $20 \%$ higher for the cylindrical support than the elliptical support due to its higher opposing thrust to the flow. Further downstream, the case with cylindrical support has higher velocity deficit followed by elliptical support and then the case with no support structure. This is because of the cylinder with higher drag presents more opposing thrust to the flow than the ellipse causing a larger drop in velocity.

In this chapter, the flow characteristics due to the interaction between the rotor and its support structure were studied. In the next chapter, the new force coefficients obtained from these CFD simulations are used for re-assessment of the initial analytical channel model. 


\section{Chapter 7}

\section{Reassessment of farm power output}

\subsection{Introduction}

In the previous chapter, the flow interaction between the rotor and the support structure was investigated in order to obtain a better estimate of the thrust forces on each of them. These force coefficients will be used in the reassessment of the farm power output using the analytical model of Chapter 2 in order to obtain a better farm power estimate in the presence of different support structures and a realistic pitch-to-feather power control strategy.

In this chapter, the influence of different factors on the opposing resistance presented to the channel are discussed: 1) thrust from the turbine rotor, 2) drag from the cylindrical and elliptical support structures. In Chapter 2, the support structure drag coefficient was assumed to be constant in the power-capping zone, but in the previous chapter it was observed that these values change as the blades are pitched and this is taken into consideration in the reassessment of the original model. In the next section, the updated turbine rotor force coefficients obtained from CFD are presented. Later these force coefficients are substituted into the analytical model and the variation in farm power output is discussed. 
Table 7.1: Force coefficients obtained from CFD for three different tip speed ratios in the presence of different support structures

\begin{tabular}{|l|c|c|c|c|c|}
\hline Support type & Rated speed & Tip speed ratio & Power coeff. & Thrust coeff. & Drag coeff. \\
\hline & $m s^{-1}$ & $\lambda$ & $\mathrm{C}_{P}$ & $\mathrm{C}_{T}$ & $\mathrm{C}_{D}$ \\
\hline \multirow{3}{*}{ No support } & \multirow{3}{*}{2.37} & 5.5 & 0.622 & 0.984 & - \\
& & 4.3 & 0.308 & 0.372 & - \\
\hline \multirow{3}{*}{ Elliptical support } & \multirow{2}{*}{2.30} & 5.1 & 0.085 & 0.112 & - \\
& & 4.3 & 0.630 & 0.996 & 0.08 \\
& & 3.1 & 0.315 & 0.385 & 0.12 \\
Cylindrical support & \multirow{2}{*}{2.25} & 5.5 & 0.091 & 0.119 & 0.17 \\
& & 4.3 & 0.639 & 1.012 & 0.15 \\
& & 3.1 & 0.095 & 0.392 & 0.21 \\
& & & & & 0.125 \\
\hline
\end{tabular}

Also, the influence of an environmental constraint on the permissible channel flow velocity reduction is discussed as in the original model.

\subsection{Turbine performance}

The experimental turbine rotor of $18 \mathrm{~m}$ diameter is considered to be installed in the same channel discussed in Chapter 2 of length, $\mathrm{L}=5 \mathrm{~km}$, width, $\mathrm{W}=1 \mathrm{~km}$, and depth, $\mathrm{h}=40 \mathrm{~m}$ with the same tidal conditions as discussed previously. The rotor tip speed ratio is held constant until the rated flow speed corresponding to a rated power of $1 \mathrm{MW}$, after which the blades are pitched to feather to maintain constant angular velocity implying a reduction in tip speed ratio.

The presence of support structures leads to an increase in the rotor power coefficient due to the increased blockage which leads to an increase in the local flow velocity, as discussed in the previous chapter. This would result in the rated power being achieved at a lower rated speed than expected. The rated flow speed of the turbine rotor without any support structure is $2.37 \mathrm{~ms}^{-1}$ but in the presence of a cylindrical support, the rated speed, due to the increased rotor power coefficient, drops by $5 \%$ to $2.25 \mathrm{~ms}^{-1}$, indicating that the rated power is achieved at lower flow speeds and the blades must start pitching earlier than 
otherwise expected; see table 7.1. Also from table 7.1, it can be observed that the rotor thrust coefficient increases in the presence of support structures and hence the thrust on the rotor blades also changes at rated flow speed. This will be an important consideration in designing the blades as the blades have to pitch at lower flow speeds and experience a different thrust than the expected value, increasing the number of fatigue load cycles on the blades.

As the flow speed increases above the rated speed, the blades are pitched to feather, maintaining a constant angular velocity resulting in a decrease in the tip speed ratio, which decreases the angle of attack on the blade aerofoil sections which in-turn reduces the thrust on the rotor and hence the thrust coefficient as discussed in Chapter 5; see table 7.1.

The drag coefficient on the support structures behind the rotor is lower than that of the support structures in uniform flow in the absence of a rotor. The presence of the turbine rotor and the shear in the flow, both play a role in lowering the drag coefficient. The presence of the rotor reduces the flow velocity on to the support structure but as the drag coefficient is defined based on the upstream flow velocity which is constant in both cases, with and without the presence of rotor, resulted in a lower drag coefficients for both circular and elliptical support structures. The presence of shear causes the average flow velocity on the support structure to be lower than that in the uniform flow case which also plays a key role in the decrease in the support structure drag coefficient.

As the tip speed ratio decreases with increasing blade pitch angles, the thrust on the rotor decreases resulting in a lower velocity deficit and hence a relative increase in the flow velocity behind the rotor on to the support structure causing an increase in the drag coefficient of the support structures; see table 7.1.

The new values obtained from CFD in table 7.1 are used in the initial analytical model of Chapter 2 and their influence on the farm power output is discussed in the next section. 


\subsection{Channel power output}

The channel dynamics model was discussed in Chapter 2 with the opposing forces from the rotor thrust, drag on the support structure and seabed friction included in the model. The final governing equation in the non-dimensional form is given by

$$
\frac{d Q^{\prime}}{d t^{\prime}}-\cos t^{\prime}=-\frac{1}{2} Q^{\prime}\left|Q^{\prime}\right| \frac{1}{F r_{w}^{2}}\left(\xi\left(C_{T}+\chi C_{D}\right)+C_{f} \frac{L}{h}\right)
$$

where $F r_{w}=\omega L / \sqrt{g a}$ is a non-dimensional grouping taking a similar form to the Froude number, $\omega$ is the frequency of the sinusoidal tide, $a$ is the tidal amplitude and $L$ is the length of the channel. The non-dimensional quantities are given by $t^{\prime}=\omega t, \xi=n A_{t} / A_{c}$ represents the ratio of total rotor frontal area to channel cross-sectional area, $A_{c}$, (note that this is not the same as the global blockage ratio as the rotors are not constrained to lie in the same plane), $Q^{\prime}=Q / Q_{0}$ (where $Q_{0}=g a / \omega c$ is the magnitude of the volume flux

in the absence of flow resistance, $c$ is the coefficient given by $\int_{0}^{L} A_{c}^{-1} d x$ ), and $\chi$ is the ratio of support structure frontal area to turbine rotor frontal area. $C_{T}, C_{D}$ and $C_{f}$ are the rotor thrust coefficient, support structure drag coefficient and seabed friction coefficient respectively.

The solution to the above equation is obtained through time marching. For all cases investigated in this work, a representative seabed friction coefficient of $C_{f}=0.002$ (Massey, 1998 ) is considered. The values of $C_{T}$ and $C_{D}$ are obtained from the CFD simulations, the values of which were presented in the previous section.

Whilst $C_{T}, C_{D}, \xi$ and $\chi$ describe the tidal energy farm, $F r_{w}, C_{f}$ and $L / h$ characterize the underlying channel flow. In this chapter, tidal energy extraction will be explored as a function of the channel parameters, $F r_{w}$ and $L / h$. Both $F r_{w}$ and $L / h$ have a dependency on $\mathrm{L}$, and hence $F r_{w}$ is varied by changing tidal amplitude, $a$, and maintaining $\mathrm{L}$ 


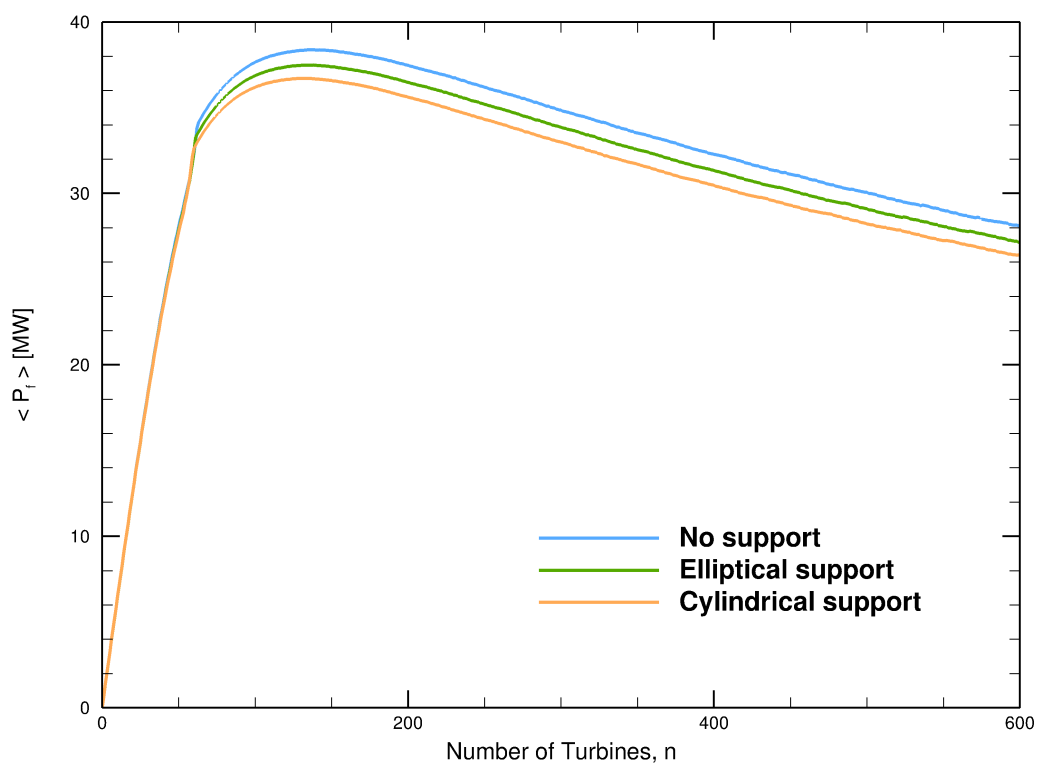

Figure 7.1: Time-averaged total farm power output, $\left\langle P_{f}\right\rangle$, as a function of the number of turbines, $\mathrm{n}$, installed and support structure drag coefficient. ( $\chi=0.16, F r_{w}=0.478$, $L / h=125, C_{f}=0.002$ )

constant. When $L / h$ is varied by changing $\mathrm{L}$, this would change $F r_{w}$ as well and hence tidal amplitude, $a$, is also varied such that $F r_{w}$ remains constant. $F r_{w}$ and $L / h$ may be viewed as indicators of the volume flow rate (or velocity) through the channel. A lower $F r_{w}$ corresponds to a higher tidal amplitude, $a$, or a shorter channel, $L$, both of which imply a larger relative head to volume of water within the channel which results in higher flow velocity. Similarly a higher $L / h$ implies a relatively shallow channel and hence the same head trying to drive a smaller volume of water resulting in a higher flow velocity.

Based on the channel dimensions described in section 7.2, $L / h=125$ and $F r_{w}=$ 0.478 for the tidal amplitude, $\mathrm{a}=0.25 \mathrm{~m}$. For these values, the equation 2.14 is evolved in time for $Q^{\prime}$ to obtain the volume flow rate, $\mathrm{Q}$, and thus the farm power and thrust are calculated. The time averaged farm power for this channel is computed for a range of number of turbines installed and different support structure shapes; see figure 7.1. The total power output from the farm increases in an almost linear manner until the decrease in 
flow rate becomes a constraining factor in the farm power output. At this point, the large decrease in flow rate results in only a small increase in the total farm power output even though more turbines are being installed. Beyond the peak point, the total farm power starts to decrease due to an even higher drop in the channel flow rate. Note that in this analysis, the flow from the turbines are assumed not to interact with each other.

In the presence of support structures, there is an increase in the opposing thrust presented to the channel resulting in a decrease in the farm power output; see figure 7.1. The peak power output drops by about $6 \%$ in the presence of the relatively lower drag elliptical support structures and by about $10 \%$ in the presence of the higher drag cylindrical support structures. Also for the higher drag support structures, the peak power output is obtained for smaller numbers of turbines. This indicates that the maximum number of turbines that can be installed in a channel is dependent on the support structure drag because of the increase in the overall resistance from the channel.

\subsection{Power vs thrust}

To analyze the farm power output in the presence of support structures for different channel characteristics, the non-dimensional power and thrust coefficients, $C_{P c}$ and $C_{T c}$, from Chapter 2 are re-introduced in this section. They are given by

$$
\begin{gathered}
C_{P c}=\frac{<P_{f}>}{\rho g a Q_{0}} \\
C_{T c}=\frac{<\left|T_{c}\right|>}{\rho g a A_{c}}
\end{gathered}
$$

where $\left\langle P_{f}>\right.$ and $<\left|T_{c}\right|>$ are the time averaged absolute values of the farm power output and the opposing channel flow resistance (rotor thrust, support structure drag and 
seabed friction) respectively. The term $\rho g a Q_{0}$ is an indicator of the power available in the channel in the absence of any kind of flow resistance. Garrett and Cummins (2005) showed that in the case of linear flow resistance the maximum power that can be generated from a tidal channel is $\frac{1}{4} \rho g a Q_{0}$, so that $C_{P c}$ becomes a measure of the power that can be generated from the channel and takes a maximum value of $1 / 4$.

The channel characteristics were non-dimensionalised in the previous section using $F r_{w}$ and $L / h$, and their influence on farm performance $C_{P c}$ and $C_{T c}$ is studied in this section. The influence of tidal amplitude on the channel power coefficient $C_{P c}$ through varying $F r_{w}$ is shown in figure 7.2. Initially when there are no turbines installed, the opposing thrust from the channel is due to seabed friction alone and hence $C_{T c}$ is nonzero even though $C_{P c}$ is zero initially. This non-zero thrust due to seabed friction can be determined from the value of $C_{T c}$ at $C_{P c}=0$; see figure 7.2. Also, $C_{T c}$ purely due to seabed friction, at $C_{P c}=0$, increases as $F r_{w}$ decreases from 0.673 to 0.338 (due to an increase in the corresponding peak channel flow velocity from $1.7 \mathrm{~ms}^{-1}$ to $5.3 \mathrm{~ms}^{-1}$ ).

$C_{P c}$ and $C_{T c}$ for the base case of $F r_{w}=0.478$ (corresponding to $a=0.25 \mathrm{~m}$ ) and $L / h=125$ are shown in figure 7.2b. As identified previously, the total power output, $C_{P c}$ is seen to decrease with an increase in the support structure drag coefficient, inferring that the parasitic power loss is higher in the presence of support structures.

As the number of turbines installed in the farm increases, the overall rotor thrust and support structure drag from the farm increases. As a consequence, the maximum channel velocity is reduced and this reduction in channel velocities can have a negative environmental impact as discussed in Chapter 2. The analysis is repeated by imposing the flow rate reduction constraints limiting the peak flow rate reduction to no more than ten and twenty percent and study the implications for $C_{P c}$. In figure $7.2 \mathrm{~b}$, there are two dashed lines with $\Delta Q / Q_{0}=10 \%$ and $20 \%$ indicating the levels of reduction in peak volume 


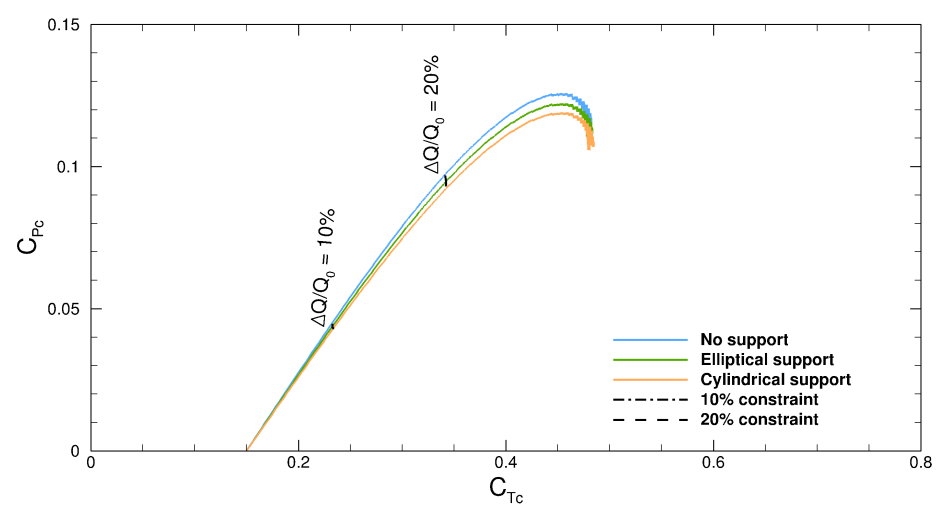

(a)

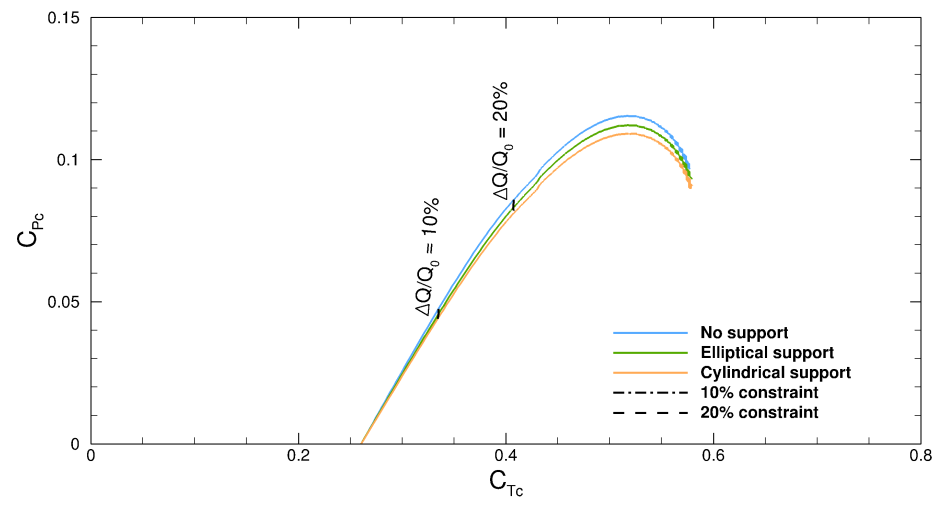

(b)

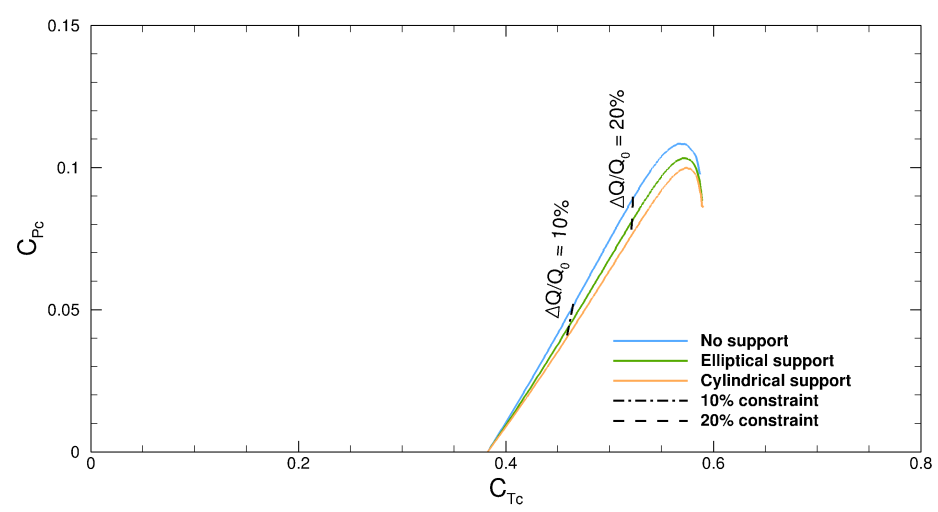

(c)

Figure 7.2: Channel power coefficient, $C_{P c}$, as a function of channel thrust coefficient, $C_{T c}$, for (a) $F r_{w}=0.673(a=0.125 m)$, (b) $F r_{w}=0.478(a=0.25 m)$, (c) $F r_{w}=0.338$ $(a=0.5 m)$. The dashed lines indicate constraints on change in the maximum volume flow rate in the channel. $\left(L / h=125, C_{f}=0.002, \chi=0.16\right)$. 


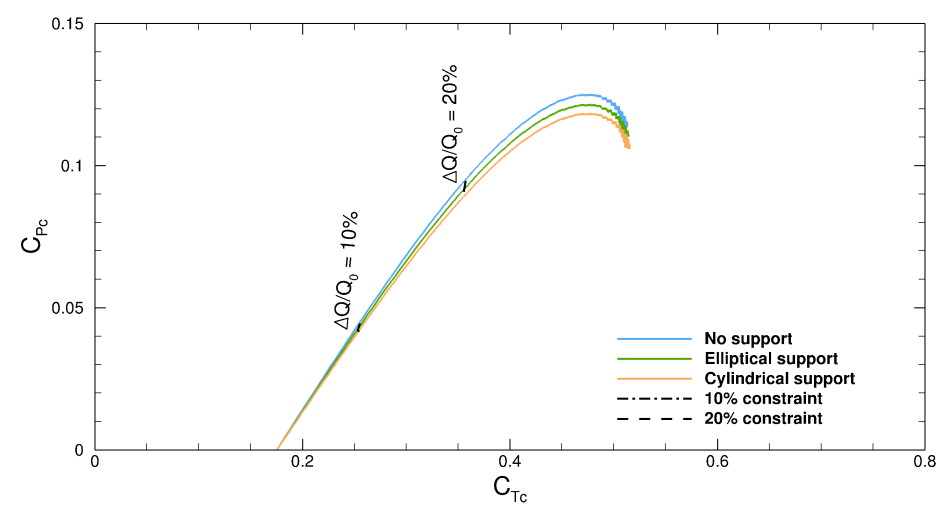

(a)

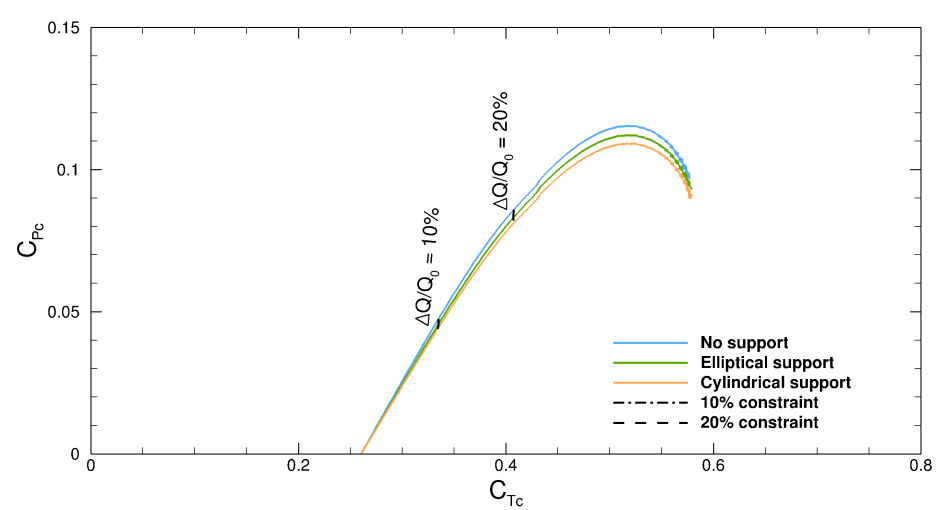

(b)

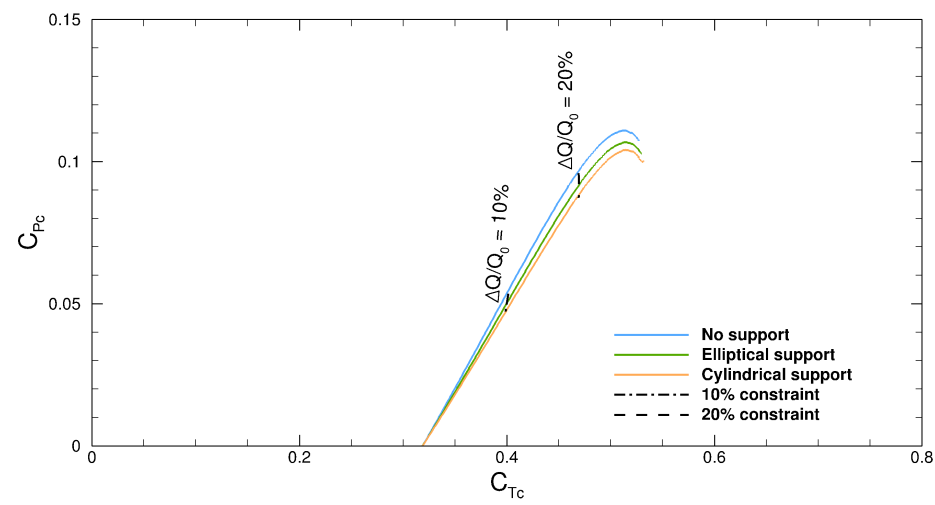

(c)

Figure 7.3: Channel power coefficient, $C_{P c}$, as a function of channel thrust coefficient, $C_{T c}$, for (a) $L / h=75$, (b) $L / h=125$, (c) $L / h=175$. The dashed lines indicate constraints on change in the maximum volume flow rate in the channel $\left(F r_{w}=0.478, C_{f}=\right.$ $0.002, \chi=0.16)$. Note that figure $7.2 \mathrm{~b}$ is the same as $7.3 \mathrm{~b}$ because they have the same $F r_{w}$ and $L / h$. 
flow rate. Even for these large permissible flow rate reductions, maximum channel power is significantly reduced below the peak achievable power in the case of no flow rate reduction constraint, and the structural drag further compounds the reduction in maximum deliverable power.

For the case of $F r_{w}=0.338(a=0.5 \mathrm{~m})$, a flow rate reduction constraint of $\Delta Q / Q_{0}=$ $10 \%$ reduces the maximum deliverable power by $54 \%$ in the case of no support structure, $C_{D}=0$, and the introduction of a cylindrical support further reduces the maximum deliverable power by $15 \%$ compared to the case with no support structure and a $10 \%$ flow rate constraint.

The other non-dimensional parameter of importance is $L / h$. Based on the dimensions of the base case channel, an $L / h$ value of 125 is obtained. A higher $L / h$ results in a higher energy flow as was observed with an increase in the flow velocity from $2 \mathrm{~ms}^{-1}$ for $L / h=75$ to $4.1 \mathrm{~ms}^{-1}$ for $L / h=175$ before the installation of any turbines in the channel. Hence, an increase in $C_{T c}$ is observed with increasing $L / h$ in the absence of turbines, i.e. due to bed friction alone, where $C_{P c}=0$; see figure 7.3. Also as $L / h$ is increased and greater energy is given up to overcome bed friction, less energy is available for turbine power generation or to overcome support structure drag, and hence peak $C_{P c}$ falls with increasing $L / h$ for all values of $C_{D}$.

\subsection{Available power in a volume flux reduction constrained flow}

In this section, the non-dimensional data studied in the previous section is analysed in dimensional terms for specific cases for a more practical interpretation. As discussed in the previous sections, different channel characteristics are studied by varying the non- 
Table 7.2: Farm power output dependency on $F r_{w}$

\begin{tabular}{|l|l|l|l|l|l|l|l|l|}
\hline Support & $F r_{w}$ & $U_{A}$ & \multicolumn{2}{|c|}{$P_{f \max }$} & \multicolumn{2}{|l|}{$P_{f \Delta Q / Q_{0}=10 \%}$} & \multicolumn{2}{l|}{$P_{f \Delta Q / Q_{0}=20 \%}$} \\
\hline & & $m s^{-1}$ & {$[\mathrm{MW}]$} & $k$ & {$[\mathrm{MW}]$} & $k$ & {$[\mathrm{MW}]$} & $k$ \\
\hline \multirow{3}{*}{ No support } & 0.673 & 1.65 & 10.2 & & 3.6 & & 7.8 & \\
& 0.478 & 3.27 & 37.1 & 1.88 & 15.2 & 1.88 & 27.6 & 1.88 \\
& 0.338 & 6.55 & 139.4 & & 66.9 & & 115.4 & \\
\hline \multirow{3}{*}{ Elliptical support } & 0.673 & 1.65 & 9.9 & & 3.5 & & 7.6 & \\
& 0.478 & 3.27 & 36.0 & 1.88 & 14.6 & 1.65 & 26.6 & 1.72 \\
& 0.338 & 6.55 & 132.9 & & 57.9 & & 105.4 & \\
\hline \multirow{4}{*}{ Cylindrical support } & 0.673 & 1.65 & 9.7 & & 3.5 & & 7.4 & \\
& 0.478 & 3.27 & 35.1 & 1.88 & 14.1 & 1.48 & 26.1 & 1.54 \\
& 0.338 & 6.55 & 128.4 & & 52.6 & & 98.2 & \\
\hline
\end{tabular}

dimensional parameters, $F r_{w}$ and $L / h$. For a corresponding non-dimensional parameter, either $F r_{w}$ or $L / h$, in the absence of any turbines, there is a maximum undisturbed channel flow velocity, $U_{A}$, and the variation of the farm power output with this maximum undisturbed channel flow velocity will be studied in this section. The variation of the total farm power output with $F r_{w}$ is shown in table 7.2, in which $P_{f \max }$ represents the peak power output for a particular $F r_{w}$ (and the corresponding undisturbed peak channel velocity, $U_{A}$ ) and support structure, and other columns represent the power output with volume flow rate constraints of $10 \%$ and $20 \%$. Also shown is the computed exponent $k$ which describes the variation of maximum power output with the undisturbed peak channel velocity; $P_{f} \propto U_{A}^{k}$.

In figure 7.4a, the peak power output is shown as a function of the peak channel velocity for a range of tidal amplitudes for different support structures. The variation of maximum power output is almost quadratic with respect to undisturbed peak flow speed (for $P_{\text {fmax }}, k \approx 1.88$; see table 7.2 ) but not exactly so due to sea bed friction (irrespective of the level of structural drag). This follows from the energy flux in the tidal wave being proportional to the square of wave amplitude and thus, in the absence of bed friction, to flow speed squared. Similarly in figure $7.4 \mathrm{~b}$, the maximum power output with a $10 \%$ volume flow rate constraint is shown and the behaviour of these curves is quite different from those of peak power. The power-velocity exponent for elliptical and cylindrical supports 


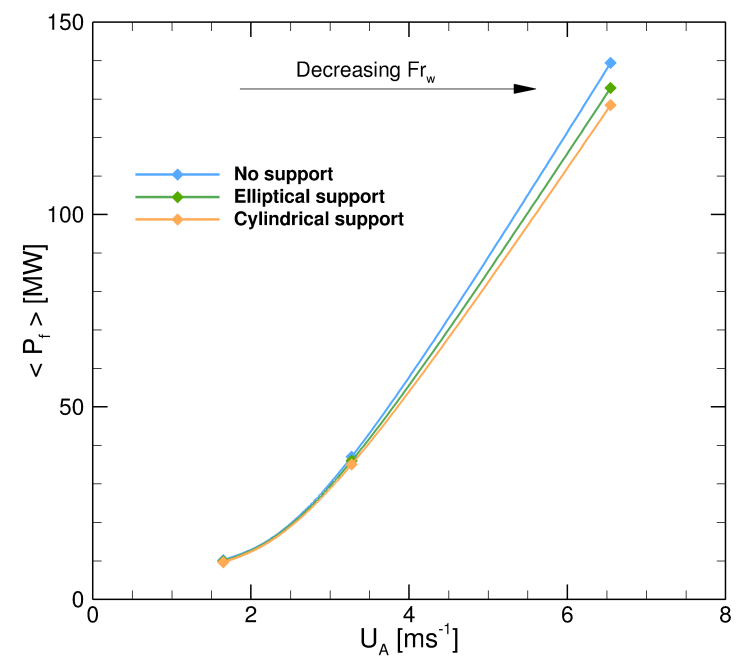

(a) Peak power

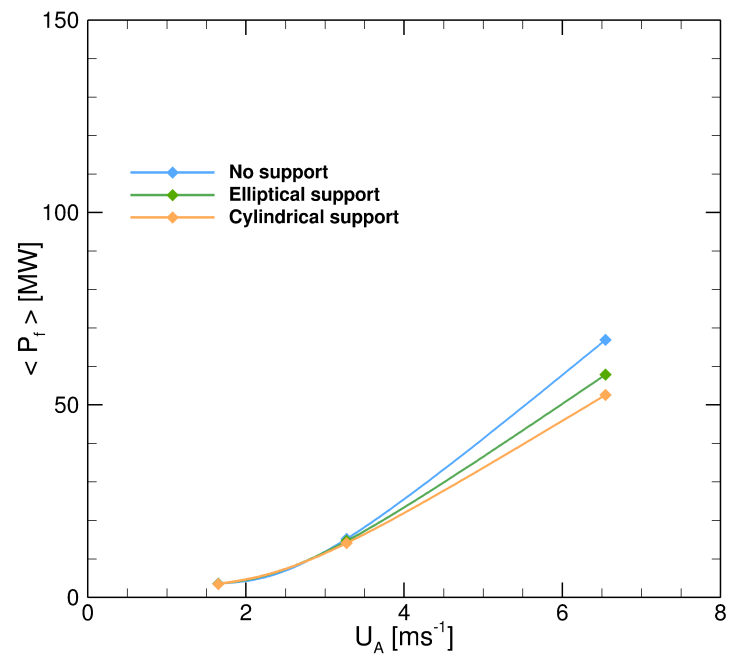

(b) Power with $10 \%$ volume flow rate constraint

Figure 7.4: Maximum undisturbed channel velocity (due to different $F r_{w}$ ) vs. the time averaged power output of the tidal farm, $\left\langle P_{f}\right\rangle$, for different support structure drag coefficients. $\left(L / h=125, C_{f}=0.002, \chi=0.16\right)$.

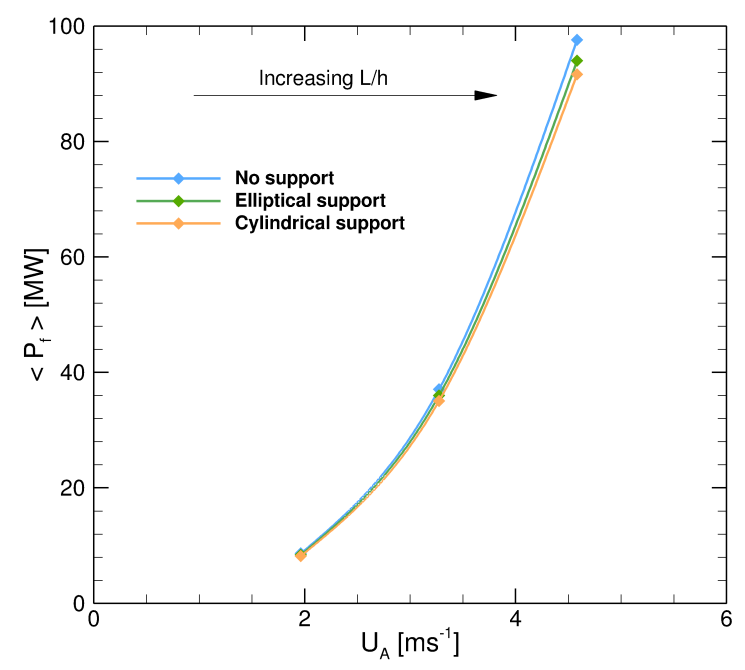

(a) Peak power

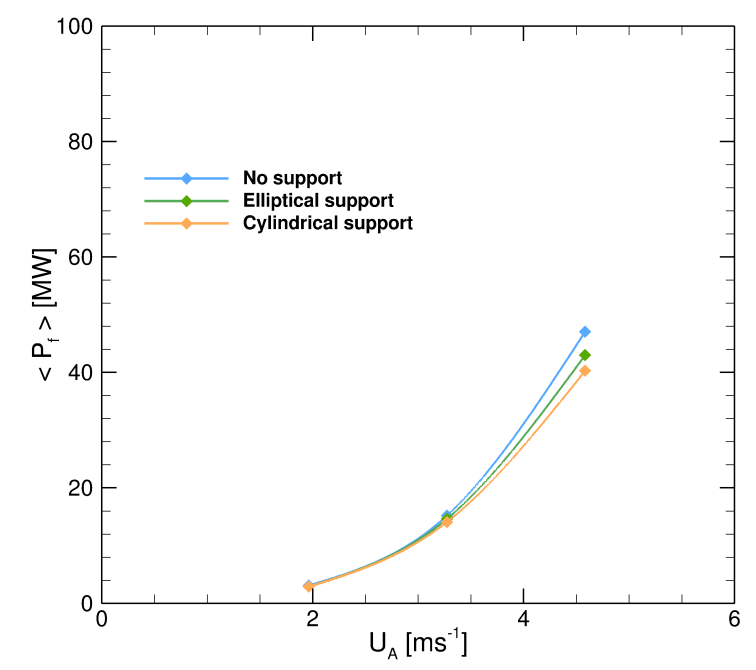

(b) Power with $10 \%$ volume flow rate constraint

Figure 7.5: Maximum undisturbed channel velocity (due to different $L / h$ ) vs. the time averaged power output of the tidal farm, $\left\langle P_{f}\right\rangle$, for different support structure drag coefficients. $\left(F r_{w}=0.478, C_{f}=0.002, \chi=0.16\right)$. 
Table 7.3: Farm power output dependency on $\mathrm{L} / \mathrm{h}$

\begin{tabular}{|c|c|c|c|c|c|c|c|c|}
\hline Support & $\mathrm{L} / \mathrm{h}$ & $U_{A}$ & $P_{f}$ & & $P_{f \Delta Q}$ & $0=10 \%$ & $P_{f \Delta Q /}$ & $0=20 \%$ \\
\hline & & $m s^{-1}$ & {$[\mathrm{MW}]$} & $k$ & [MW] & $k$ & [MW] & $k$ \\
\hline & 75 & 1.96 & 8.7 & & 3.1 & & 6.6 & \\
\hline No support & 125 & 3.27 & 37.0 & 2.85 & 15.2 & 2.85 & 27.6 & 2.85 \\
\hline & 175 & 4.58 & 97.6 & & 47.0 & & 84.4 & \\
\hline & 75 & 1.96 & 8.4 & & 2.9 & & 6.3 & \\
\hline Elliptical support & 125 & 3.27 & 36.0 & 2.85 & 14.6 & 2.68 & 26.6 & 2.72 \\
\hline & 175 & 4.58 & 93.9 & & 42.9 & & 79.9 & \\
\hline & 75 & 1.96 & 8.2 & & 2.9 & & 6.2 & \\
\hline Cylindrical support & 125 & 3.27 & 35.0 & 2.85 & 14.1 & 2.59 & 26.1 & 2.62 \\
\hline & 175 & 4.58 & 91.6 & & 40.3 & & 76.7 & \\
\hline
\end{tabular}

are markedly reduced to 1.65 and 1.48 respectively and for $\Delta Q / Q_{0}=20 \%$, the exponents are 1.72 and 1.54 respectively (see table 7.2 ). Thus, for the channel considered, for a realistic flow rate reduction constraint $(10 \%)$ and cylindrical support $(\chi=0.16)$, the farm power variation exponent with $U_{A}$, the undisturbed peak flow velocity, is $20 \%$ lower than the case with no support and no volume flow rate constraint, when this is controlled through altering $F r_{w}$. For a fixed geometry channel, different undisturbed peak flow velocities result from different amplitude tidal waves (and thus $F r_{w}$ ). For the channel considered, in the absence of support structures $\left(C_{D}=0\right)$, peak power output for $a=0.5 m\left(F r_{w}=0.338\right)$ is around $140 \mathrm{MW}$ but in the presence of a volume flow rate constraint (10\%), the power output drops by $50 \%$ to $67 \mathrm{MW}$ and in the presence of a cylindrical support $(\chi=0.16)$, the power output drops further to 53MW.

The influence of the other non-dimensional parameter, $L / h$ on the variation of the total power output is shown in table 7.3. Similar plots are studied for a range of $L / h$ values in dimensional space; see figure 7.5. The variation in peak power output is a little less than cubic in flow speed (due to bed friction), see table 7.3, when flow speed is controlled through $L / h$. The presence of support structures reduces the power-velocity exponent, but less markedly so than when $F r_{w}$ is used to control $U_{A}$. 
Table 7.4: Influence of support structure drag on $C_{P c}$ with a $10 \%$ volume flow rate reduction constraint for different $F r_{w}$

\begin{tabular}{|c|c|l|c|}
\hline$F r_{w}$ & $U_{A}\left(\mathrm{~ms}^{-1}\right)$ & Support type & $C_{P c \triangle Q / Q_{0}}=10 \%$ \\
\hline \multirow{3}{*}{0.673} & \multirow{3}{*}{1.65} & No support & 0.051 \\
& & Elliptical support & 0.049 \\
& & Cylindrical support & 0.046 \\
\hline \multirow{3}{*}{0.478} & \multirow{3}{*}{3.27} & No support & 0.050 \\
& & Elliptical support & 0.047 \\
& & Cylindrical support & 0.045 \\
\hline \multirow{3}{*}{0.338} & \multirow{3}{*}{6.55} & No support & 0.049 \\
& & Elliptical support & 0.044 \\
& & Cylindrical support & 0.041 \\
\hline
\end{tabular}

\subsection{Influence of support structure drag}

The influence of imposing a volume flow constraint in the channel was discussed in the previous section. In this section, a $10 \%$ volume flow rate reduction constraint is imposed, and the influence of the support structure drag coefficient on $C_{P c}$ is analysed. In figure $7.4 \mathrm{~b}$, it was observed that the power output is higher for higher flow speed (lower $F r_{w}$ ) and increases less than quadratically with flow speed. However, once non-dimensionalized, the power coefficient, $C_{P c}$, is observed to decrease with increasing flow speed (lower $F r_{w}$ ); see table 7.4. This indicates that the proportion of channel power that can be realised as useful power decreases as tidal amplitude increases due to increasing bed friction losses. Also, as the support structure drag increases, there is a drop in $C_{P c}$ and the drop is about $10 \%$ for $F r_{w}=0.478$ when compared between no support and cylindrical support; see table 7.4. The same drop for $F r_{w}=0.338(a=0.5 \mathrm{~m})$ is much higher at $16 \%$ because at higher tidal amplitudes, the velocity is higher and hence the impact of support structure drag is also higher which leads to a sharp decrease in $C_{P c}$.

The influence of the non-dimensional parameter $L / h$ was studied in a similar manner by plotting $C_{P c}$ against $C_{D}$; see table 7.5. As $L / h$ is increased (with $F r_{w}$ maintained at 
Table 7.5: Influence of support structure drag on $C_{P c}$ with a $10 \%$ volume flow rate reduction constraint for different $\mathrm{L} / \mathrm{h}$

\begin{tabular}{|c|c|l|c|}
\hline $\mathrm{L} / \mathrm{h}$ & $U_{A}\left(\mathrm{~ms}^{-1}\right)$ & Support type & $C_{P c \Delta Q / Q_{0}=10 \%}$ \\
\hline \multirow{3}{*}{75} & \multirow{3}{*}{1.96} & No support & 0.052 \\
& & Elliptical support & 0.049 \\
& & Cylindrical support & 0.046 \\
\hline \multirow{3}{*}{125} & \multirow{3}{*}{3.27} & No support & 0.050 \\
& & Elliptical support & 0.047 \\
& & Cylindrical support & 0.045 \\
\hline \multirow{3}{*}{175} & \multirow{3}{*}{4.58} & No support & 0.049 \\
& & Elliptical support & 0.046 \\
& & Cylindrical support & 0.044 \\
\hline
\end{tabular}

$0.478), C_{P c}$, increases due to the higher energy flow. As the support structure drag coefficient increases, $C_{P c}$ decreases by about $10 \%$ for $L / h=125$ when compared between no support and cylindrical support. This drop is even higher at $12 \%$ for $L / h=175$ due to the larger impact of support structures in a higher velocity flow as discussed earlier in the case of $F r_{w}$.

\subsection{Conclusion}

The channel dynamics model of Chapter 2 has been revisited in this chapter with more precise data obtained from the CFD simulations in the previous chapters. The influence of support structure drag and turbine rotor thrust on the overall farm power output has been investigated in this chapter. As the number of turbines installed in the channel increased, the increase in resistance in the channel reduced the flow rate, with the consequence that the marginal power of additional turbines becomes negative and the installation of further turbines becomes detrimental to farm power output.

For the base case channel considered, compared to the case with no support structures, in the presence of elliptical support structures, the peak power dropped by $6 \%$ and in 
the presence of cylindrical support structures, it dropped by $10 \%$. This is lower than the upper bound presented in Chapter 2 wherein the total opposing turbine thrust was obtained from the super-position of rotor and support structure thrust without considering any interaction effects as in the present case. Due to the interaction between the rotor and the support structure causing a decrease in the velocity of approach onto the support structure, the drag on the support structure is considerably lower than that assumed in Chapter 2. Hence the total turbine system thrust is lower than the assumed thrust value obtained from superposition of rotor and support structure thrust. Hence its influence on farm power output is not as high as discussed in Chapter 2 but still sufficiently high to be considered while designing turbine support structures.

The non-dimensional parameters $F r_{w}$ and $L / h$ were used to study the influence of channel characteristics on farm power output and overall thrust. For different $F r_{w}$, the farm power output varied a little less than quadratically with the undisturbed channel flow velocity with the sea bed friction causing a deviation from the quadratic behaviour. This follows from the energy flux in the tidal wave being proportional to the square of wave amplitude and thus, in the absence of bed friction, to flow speed squared. Similarly for different $L / h$, the variation of farm power output with undisturbed channel flow velocity is little less than cubic. In the presence of cylindrical support structures, for $F r_{w}=0.338$ and $L / h=125$, the drop in the maximum power was as high as $16 \%$ indicating the importance of support structures.

Later a volume flow rate constraint was imposed on the reduction in channel velocity to minimise disruption to the environment. With this constraint, the order of farm power output variation with the undisturbed channel velocity decreased indicating that the achievable power would be greatly reduced with environmental constraints.

The computations in the previous few chapters provided a good dataset which takes 
into consideration the interaction effects between the rotor and its support structure. The reassessment of the analytical model in Chapter 2 with the new dataset resulted in better estimates of the farm power output which are in within the upper-bound values presented in Chapter 2. 


\section{Chapter 8}

\section{Conclusions}

This thesis discusses the importance of tidal turbine support structures through analytical and computational modelling. The analytical channel model was used to study the global effect by estimating the total farm power output and the computational model was used to study the device scale interactions between the turbine rotor and its support structure. The computational simulations were performed using the open-source CFD solver OpenFOAM.

The channel model was analyzed in Chapter 2 to test the importance of undertaking the study on support structures. It showed that there could potentially be a significant reduction in farm power output, even though only approximate force coefficients of rotor and support structure were considered. To advance this, the computational simulations were performed at device scale to obtain accurate force coefficients considering the rotorsupport structure interactions. This dataset was used to reassess the farm power output using the channel model to indicate the importance of support structures.

This chapter is structured such that the results from shear flow and blade pitching analysis are summarised first. The conclusions about the rotor-support structure interaction are discussed in the next section. Later, the influence of the support structures on the overall 
turbine farm power are discussed. The last section discusses possibilities for future work in this area.

\subsection{Conclusions}

\subsubsection{Influence of shear flow and blade pitching on turbine perfor- mance}

A full-scale tidal turbine, 1MW TGL rotor, was simulated using blade resolved CFD to analyze the influence of a shear velocity profile and blade pitching, in the power-capping zone, on rotor power and thrust, and on wake evolution.

The force coefficients in shear flow were found to be higher due to the higher average velocity flow in the turbine swept area compared to the uniform flow, with both cases having the same bulk channel flow velocity of $2 \mathrm{~ms}^{-1}$. The loading on the rotor blade during a rotation in a shear flow is azimuthally asymmetric as compared to the symmetric loading in a uniform flow. This unsteady loading in the shear flow will likely reduce the fatigue life of the blades.

The angle of attack along the span of the blade varied from $5^{\circ}$ to $8^{\circ}$ at the design tip speed ratio of 5.5 which produces the optimum lift to drag ratio for the aerofoil sections used in the blade.

The influence of pitch-to-feather power capping strategy was examined by studying the forces and angle of attack on the turbine blade, and the wake at three different blade pitch angles; $0^{\circ}, 10^{\circ}$ and $20^{\circ}$. The flow angle relative to rotor plane increases at higher blade pitch angles due to the decreasing rotor thrust causing an increase in the axial flow velocity through the rotor. However, due to blade pitching, the angle of attack on the blades decreases even though the flow angle relative to rotor plane increases, thereby reducing 
the power coefficient and maintaining constant power in the power capping zone. The maximum angle of attack on the blade tip $(r / R=0.9)$ during blade rotation decreases from about $6^{\circ}$ for $0^{\circ}$ blade pitch angle case to about $0^{\circ}$ for $20^{\circ}$ blade pitch angle case.

The decreasing angle of attack on the blade at higher blade pitch angles results in a significant drop in the average load on the blade. The loading distribution on the blade is quite different for all the three blade pitch angle cases with the point of maximum axial load along the blade span shifting from $r / R=0.85$ (closer to the tip) for $0^{\circ}$ blade pitch angle to $r / R=0.35$ (closer to the root) for $20^{\circ}$ blade pitch angle.

The change in rotor thrust, due to changing blade pitch angle, has a direct influence on the wake evolution behind the rotor. An increase in blade pitch angle increases the rate of velocity recovery in the wake due to lower turbine rotor thrust. This also resulted in a decrease in the wake width and a decrease in the tip vortex strength. Furthermore, the turbulence intensity in the wake decreases with increasing blade pitch angle due to the relatively lower shear between the bypass flow and the flow in the wake.

\subsubsection{Rotor-support structure interaction}

The full-scale tidal turbine was simulated in the shear flow in the presence of two different support structures, circular and elliptical, to study the flow interaction between the rotor and its support structure for different blade pitch angles, and the comparison between different support structures for the $10^{\circ}$ blade pitch angle was discussed in detail.

The integrated rotor force coefficients were higher in the presence of the cylindrical support structure than the elliptical support due to the higher opposing thrust from the cylinder in the channel diverting the flow and increasing the local flow velocity near the top half of the rotor.

The variation in the force coefficients over a single blade rotation were also higher 
in the presence of support structures as they influence the pressure distribution around the blade when it passes in front of the support structure. As a result, the minimum in the force coefficient does not occur at the exact bottom, $180^{\circ}$. The minimum in the power coefficient occurs at $166^{\circ}$ and $170^{\circ}$ for cylinder and ellipse respectively whereas the minimum in the thrust coefficient occurs at $170^{\circ}$ and $175^{\circ}$.

The angle of attack on the blade decreases as it approaches the support structure due to the reduction in streamwise flow velocity just ahead of the support structure. This drop is higher for the cylindrical support than for the elliptical support due to its larger opposing thrust on the flow causing a larger drop in streamwise velocity. The drop in the angle of attack on the blade due to the presence of support structures causes a significant drop in the blade sectional forces as they approach the support structure.

The presence of the rotor also causes a drop in the forces on the support structure. The mean streamwise force coefficients, non-dimensionalized based on the average inlet flow velocity, of the circular and elliptical support structures were 0.21 and 0.12 respectively, which are much lower than if they were in open water without a rotor. The presence of the rotor reduces the approach flow velocity on the support structures which reduces the streamwise force on them. The amplitude of the streamwise sectional forces was also lower in the case of an ellipse than the cylinder due to the more streamlined shape of ellipse delaying the flow separation. The mean sectional streamwise force on elliptical support on a section $(z / R=1.2)$ behind the blade tip, at bottom position, was $50 \%$ lower than that in the case of cylindrical support.

The swirl from the rotor induces a mean cross-stream force component on the support structure which is much higher in the case of an ellipse than the cylinder. This is because a small change in the angle of attack causes a large change in the forces on the ellipse than on the cylinder. The mean sectional cross-stream force on elliptical support on a section 
$(z / R=1.2)$ behind the blade tip, at bottom position, was about 2.5 times that in the case of cylindrical support. This might prove to be a significant consideration in designing an elliptical support.

The combined thrust from the rotor and the support structure has a direct influence on the wake velocity. The case with a cylindrical support has a higher wake velocity deficit followed by the elliptical support structure case and then the case with no support structure. This is because the cylinder with higher drag presents more opposing thrust to the flow than the ellipse, causing a larger drop in velocity.

\subsubsection{Tidal farm power output}

The computational modelling of turbines at device scale provided the rotor and support structure force coefficients as a function of incident flow speed in the power-capping zone, using pitch-to-feather strategy. This dataset was used in the channel model, where the channel velocity varies in a range, to estimate the overall power output from a tidal farm. A generalised rectangular channel of $5 \mathrm{~km}$ length, $1 \mathrm{~km}$ width and $40 \mathrm{~m}$ depth was considered for this analysis.

As the number of turbines installed in the channel increased, the increase in channel resistance reduced the flow rate. This results in a decrease in the power output per turbine for every additional turbine added and after a certain threshold in the number of turbines installed, the channel velocity drops so low that it results in a reduction in the overall farm power output thereafter.

The presence of support structures also reduced the farm power output. For the base case channel considered, compared to the case with no support structures, in the presence of elliptical support structures, the peak power dropped by $6 \%$ and in the presence of cylindrical support structures, it dropped by $10 \%$. 
The non-dimensional parameters $F r_{w}$ and $L / h$, which represent the channel characteristics were used to study the farm power output and overall thrust. For different $F r_{w}$, the farm power output varied a little less than quadratically with the undisturbed channel flow velocity with the sea bed friction causing a deviation from the quadratic behaviour. This follows from the energy flux in the tidal wave being proportional to the square of wave amplitude and thus, in the absence of bed friction, to flow speed squared. Similarly for different $L / h$, the variation of farm power output with undisturbed channel flow velocity is a little less than cubic.

Later a volume flow rate constraint was imposed on the reduction in channel velocity to limit disruption to the environment. With this constraint, the exponent of farm power output variation with the undisturbed channel velocity decreased indicating that the achievable power would be greatly reduced with environmental constraints. With a $10 \%$ flow rate constraint, the realizable power output dropped by $50 \%$ in the case of no support structures, and by $58 \%$ and $65 \%$ overall in the presence of elliptical support and cylindrical support respectively.

Overall an increase in the drag of the support structures resulted in a significant drop in the farm power output and lower drag support structures should be considered in the future.

\subsection{Future Work}

The inlet flow in this thesis was always assumed to be at $0^{\circ}$ yaw angle which may not be the case in all the tidal channels and it might vary up to $15^{\circ}$ in certain tidal channels. The influence of yaw on tidal turbine rotor and its support structure could be examined, particularly in the case of elliptical support where the angle of attack on the ellipse would be an important factor in determining the forces on the support structure. 
The tidal turbine support structures discussed in this work were mounted with a single rotor but in some cases such as the SeaGen turbine, two rotors might be mounted on a single support structure. The rotor-support structure interaction might be quite different in this case as the support structure is not directly in the swirling wake of the rotors and hence the forces on the support structure, both streamwise and cross-stream, would be different, and could be analyzed.

The power output from the tidal farm was obtained using a simple analytical in the present work. Using the CFD data from this thesis, more advanced models such as shallow water equations can be used to obtain an even better estimate of the power output from the farm.

RANS model was used all through this research to reduce the computational cost. RANS model may not be sufficient to study the far wake properties and LES is known to be better at predicting this. The high computational cost of LES makes it infeasible to perform these simulations in the near future but a hybrid RANS-LES model might be used in the near future given the increasing computational power everyday.

The influence of surface waves was not considered in the simulations of tidal turbines due to computational constraints. The waves might play a bigger role in the turbine power output depending on the wavelength of the wave. This might be an interesting aspect to explore in the future.

The sectional forces and the root bending moment on the rotor blades in the powercapping zone at different pitch angles was discussed in this thesis. Also the variation in the blade forces as a blade completes one rotation in shear flow was discussed. This data can be used in the fatigue analysis and other structural calculations to design the turbine blades accounting for shear profile and pitching of the blades.

The forces on the support structure in the rotor wake was different from the support 
structures in open water. This is an important consideration for design of support structures as the size of the support structure can now be reduced if they experience lower forces behind the rotor. Also in the case of elliptical support, the cross-stream force determine the structural slenderness of the ellipse which could be examined. 


\section{References}

Abbott, I. H. and von Doenhoff, A. E. (1959). Theory of Wing Sections, Including a Summary of Airfoil Data. Courier Dover Publications.

Achenbach, E. (1968). Distribution of local pressure and skin friction around a circular cylinder in cross-flow up to $\operatorname{Re}=5 \times 10^{6}$. Journal of Fluid Mechanics, 34, 625-639. ISSN 1469-7645.

Adcock, T. A. A., Draper, S., Houlsby, G. T., Borthwick, A. G. L. and Serhadlioglu, S. (2013). The available power from tidal stream turbines in the Pentland Firth. Proceedings of the Royal Society A: Mathematical, Physical and Engineering Science, 469, 20130072.

Afgan, I., McNaughton, J., Rolfo, S., Apsley, D., Stallard, T. and Stansby, P. (2013). Turbulent flow and loading on a tidal stream turbine by LES and RANS. 43, 96-108.

Ahmed, U., Apsley, D., Afgan, I., Stallard, T. and Stansby, P. (2017). Fluctuating loads on a tidal turbine due to velocity shear and turbulence: Comparison of CFD with field data. Renewable Energy, 112, 235 - 246. ISSN 0960-1481.

Ammara, I., Leclerc, C. and Masson, C. (2002). A Viscous Three-Dimensional Differential/Actuator-Disk Method for the Aerodynamic Analysis of Wind Farms. 124. 
Bahaj, A., Batten, W. and McCann, G. (2007a). Experimental verifications of numerical predictions for the hydrodynamic performance of horizontal axis marine current turbines. Renewable Energy, 32, 2479-2490.

Bahaj, A. S., Molland, A. F., Chaplin, J. R. and Batten, W. M. J. (2007b). Power and thrust measurements of marine current turbines under various hydrodynamic flow conditions in a cavitation tunnel and a towing tank. Renewable Energy, 32, 407-426.

Batten, W. M. J., Harrison, M. E. and Bahaj, A. S. (2013). Accuracy of the actuator disc-RANS approach for predicting the performance and wake of tidal turbines. Philosophical Transactions of the Royal Society of London A: Mathematical, Physical and Engineering Sciences, 371. ISSN 1364-503X.

Belloni, C. S. K. (2013). Hydrodynamics of ducted and open-centre tidal turbines. Ph.D. thesis, University of Oxford, UK.

Betz, A. (1920). Das Maximum der theoretisch moglichen Ausnutzung des Windes durch Windmotoren. Zeitschrift für das gesamte Turbinenwesen, 26, 307-309.

Blevins, R. (1990). Flow-induced vibration. Van Nostrand Reinhold International.

Brown (2009). Tidal streams and tidal stream energy device design. Technical report, Carbon Trust: London.

Carbon Trust (2011). UK Tidal Current Resource and Economics. Technical report, Carbon Trust.

Catalano, P., Wang, M., Iaccarino, G. and Moin, P. (2003). Numerical simulation of the flow around a circular cylinder at high Reynolds numbers. International Journal of Heat and Fluid Flow, 24, 463-469. 
Churchfield, Matthew, J., Li, Y., Moriarty and Patrick, J. (2013). A large-eddy simulation study of wake propagation and power production in an array of tidal-current turbines. Philosophical Transactions of the Royal Society of London A: Mathematical, Physical and Engineering Sciences, 371. ISSN 1364-503X.

Delany, N. K. and Sorensen, N. E. (1953). Low speed drag of cylinders of various shapes. Technical report, National Advisory Committee for Aeronautics.

Deparis, V., Legros, H. and Souchay, J. (2013). Tides in astronomy and astrophysics, Chapter 2: Investigations of Tides from the Antiquity to Laplace. Berlin: SpringerVerlag.

Dikshit, A. K. (1970). On the unsteady aerodynamics of stationary elliptic cylinders during organised wake condition. Ph.D. thesis, University of British Columbia, Canada.

Dolan, D. S. L. and Lehn, P. W. (2006). Simulation Model of Wind Turbine 3p Torque Oscillations due to Wind Shear and Tower Shadow. IEEE TRANSACTIONS ON ENERGY CONVERSION, 21, 717-724.

Draper, S., Stansby, P., Way, S., Adcock, T. and Stallard, T. (2013). Laboratory scale experiments and preliminary modelling to investigate basin scale tidal stream energy extraction. 10th European Wave and Tidal Energy Conference.

Drela, M. (1989). Low Reynolds Number Aerodynamics: Proceedings of the Conference Notre Dame, Indiana, USA, 5-7 June 1989, chapter XFOIL: An Analysis and Design System for Low Reynolds Number Airfoils. Springer Berlin Heidelberg, Berlin, Heidelberg, 1-12.

Elghali, S. E. B., Benbouzid, M. E. H. and Charpentier, J. F. (2007). Marine Tidal 
Current Electric Power Generation Technology: State of the Art and Current Status. In 2007 IEEE International Electric Machines Drives Conference, volume 2. 1407-1412.

Fan, R. J., Chaplin, J. R. and Yang, G. J. (2010). CFD Investigation of Performance for Marine Current Turbine Based on RANS Simulations. scientific.net, 309âĂŞ312.

Farrell, P. E. and Maddison, J. R. (2011). Conservative interpolation between volume meshes by local Galerkin projection. Computer Methods in Applied Mechanics and Engineering, 200, 89-100.

Ferrer, E. and Munduate, X. (2007). Wind turbine blade tip comparison using CFD. Journal of Physics: Conference Series, 75, 012005.

Fleming, C. (2014). Tidal Turbine Performance in the Offshore Environment. Ph.D. thesis, University of Oxford, UK.

Fleming, C., McIntosh, S. C. and Willden, R. H. J. (2013). Tidal turbine performance in sheared flow. In Proc. 10th European Wave and Tidal Energy Conference. 147 - 158.

Fruh, W.-G., Seume, J. and Gomez, A. (2008). Modelling the aerodynamic response of a wind turbine blade passing in front of the tower. In European Wind Energy Conference. $57-65$.

Funke, S., Kramer, S. and Piggott, M. (2016). Design optimisation and resource assessment for tidal-stream renewable energy farms using a new continuous turbine approach. Renewable Energy, 99, 1046 - 1061. ISSN 0960-1481.

Gant, S. and Stallard, T. (2008). Modelling a Tidal Turbine in Unsteady Flow. Proceedings of the Eighteenth (2008) International Offshore and Polar Engineering Conference, $473-479$. 
Garrett, C. and Cummins, P. (2005). The power potential of tidal currents in channels. Proceedings of the Royal Society A: Mathematical, Physical and Engineering Science, 461, 2563-2572.

Garrett, C. and Cummins, P. (2008). Limits to tidal current power. Renewable Energy, 33, 2485-2490.

Graham, J. M. R. (1976). Turbulent flow past a porous plate. Journal of Fluid Mechanics, 73, 565-591.

Gunn, K. and Stock-Williams, C. (2013). On validating numerical hydrodynamic models of complex tidal flow. International Journal of Marine Energy, 3, e82 - e97. ISSN 2214-1669. Special Issue - Selected Papers - EWTEC2013.

Heddleson, C. F., Brown, D. L. and Cliffe, R. T. (1957). Summary of Drag coefficients of various shaped cylinders. Technical report, General Electric.

Herrig, J., Emery, J. C. and Erwin, J. R. (1951). Effect of Section Thickness and Trailing Edge Radius on the Performance of NACA 65-Series Compressor Blades in Cascade at Low Speeds. Technical report, NASA RM L51J16.

Hicks (2006). Understanding Tides. Technical report, National Oceanic and Atmospheric Administration, US.

Hu, D., Hua, O. and Du, Z. (2006). A study on stall-delay for horizontal axis wind turbine. Renewable Energy, 31, 821 - 836. ISSN 0960-1481.

Hunt, J. C. R., Wray, A. A. and Moin, P. (1988). Eddies, Streams, and Convergence Zones in Turbulent Flows . Technical report, Center for Turbulence Research Report,.

ICEM-CFD (2010). ICEM CFD User Manual 15. ANSYS. 
Jasak, H. (1996). Error Analysis and Estimation for the Finite Volume Method with Applications to Fluid Flows. Ph.D. thesis, Imperial College London, UK.

Jones, W. P. and Launder, B. E. (1972). The Prediction of Laminarization with a TwoEquation Model of Turbulence. International Journal of Heat and Mass Transfer, 15, $301-314$

Joukowsky, N. (1920). Windmill of the NEJ type. Transactions of the Central Institute for Aero-Hydrodynamics of Moscow.

Komar, P. (1998). Beach Processes and Sedimentation. Prentice Hall. ISBN 9780137549382.

Kulunk, E. (2011). Aerodynamics of Wind Turbines, Fundamental and Advanced Topics in Wind Power. InTech.

Labbe, D. and Wilson, P. (2007). A numerical investigation of the effects of the spanwise length on the 3-D wake of a circular cylinder. Journal of Fluids and Structures, 23, 1168 - 1188. ISSN 0889-9746.

Lanchester, F. (1915). A contribution to the theory of propulsion and the screw propeller. Transactions of the Institute of Naval Architects, 57, 98-116.

Laplace (1782). Thórie des attractions des sphéroides et de la figure de la terre. Mém Académie Sciences Paris, 341 - 419.

Launder, B. E., Reece, G. J. and Rodi, W. (1975). Progress in the Development of a Reynolds-Stress Turbulent Closure. Journal of Fluid Mechanics, 68, 537-566.

Launder, B. E. and Sharma, B. I. (1974). Application of the Energy Dissipation Model 
of Turbulence to the Calculation of Flow Near a Spinning Disc. Letters in Heat and Mass Transfer, 1, $131-138$.

Lei, C., Cheng, L. and Kavanagh, K. (2001). Spanwise length effects on threedimensional modelling of flow over a circular cylinder. Computer Methods in Applied Mechanics and Engineering, 190, 2909 - 2923.

Lu, Y. and Lueck, R. (1999a). Using a broadband ADCP in a tidal channel. Part II: Turbulence. 16, 1568-1579.

Lu, Y. and Lueck, R. (1999b). Using broadband ADCP in a tidal channel. Part I: Mean flow and shear. 16, 1556-1567.

Malki, R., Masters, I., Williams, A. J. and Croft, T. N. (2011). The variation in wake structure of a tidal stream turbine with flow velocity. In International Conference on Computational Methods in Marine Engineering. 137 - 148.

Marine Current Turbines (2011). SeaGen Environmental Monitoring Programme Final Report. Technical report, Bristol: Marine Current Turbines.

Mason-Jones, A., O’Doherty, D. M., Morris, C. E. and O'Doherty, T. (2013). Influence of a velocity profile and support structure on tidal stream turbine performance. Renewable Energy, 52, 23-30.

Massey, B. (1998). Mechanics of Fluids. Taylor \& Francis.

Mathis, R., Marusic, I., Cabrit, O., Jones, N. L. and Ivey, G. N. (2014). Modeling bed shear-stress fluctuations in a shallow tidal channel. Journal of Geophysical Research: Oceans, 119, 3185-3199. ISSN 2169-9291. 
Mathis, R., Marusic, I., Chernyshenko, S. I. and Hutchins, N. (2013). Estimating wallshear-stress fluctuations given an outer region input. Journal of Fluid Mechanics, 715, $163 \hat{a} \mathrm{~A} S ̧ 180$.

McNaughton, J. (2013). Turbulence Modelling in the near-field of an axial flow tidal turbine using Code Saturne. Ph.D. thesis, University of Manchester, UK.

McSherry, R., Grimwade, J., Jones, I., Mathias, S., Wells, A. and Mateus, A. (2011). 3D CFD modelling of tidal turbine performance with validation against laboratory experiments.

Menter, F. R. (1993). Zonal two equation $k-\omega$ turbulence models for aerodynamic flows. In Proceedings of AIAA 24th FLuid Dynamics Conference.

Menter, F. R. (1994). Two-Equation Eddy-Viscosity Turbulence Models for Engineering Applications. AIAA Journal, 32, 1598-1605.

Mikkelsen, R. (2003). Actuator Disc Methods Applied to Wind Turbines. Ph.D. thesis, Technical University of Denmark, Denmark.

Munson, B. R., Young, D. F. and Okiishi, T. H. (2002). Fundamentals of Fluid Mechanics. John Wiley \& Sons.

Murray, R. O. and Gallego, A. (2017). A modelling study of the tidal stream resource of the Pentland Firth, Scotland. Renewable Energy, 102, 326 - 340. ISSN 0960-1481.

Myers, L. and Bahaj, A. S. (2009). Near wake properties of horizontal axis marine current turbines. In Proceedings of the 8th European Wave and Tidal Energy Conference. $67-79$. 
Norberg, C. (2003). Fluctuating lift on a circular cylinder: review and new measurements. Journal of Fluids and Structures, 17, 57-96.

O’Doherty, T., Mason-Jones, A., M O’doherty, D., B Byrne, C., Owen, I. and Wang, Y. (2009). Experimental and Computational Analysis of a Model Horizontal Axis Tidal Turbine.

Olczak, A., Stallard, T., Feng, T. and Stansby, P. (2016). Comparison of a RANS blade element model for tidal turbine arrays with laboratory scale measurements of wake velocity and rotor thrust. Journal of Fluids and Structures, 64, 87 - 106. ISSN 0889-9746.

OpenFOAM (2015). OpenFOAM User Guide. Technical report, OpenFOAM Foundation.

Orme, A. C. and Masters, I. (2006). Analysis and comparison of support structure concepts for tidal stream turbines. Proc. World Maritime Technology Conference.

Patankar, S. V. (1981). Numerical heat transfer and fluid flow. Hemisphere Publishing Corporation.

Plumb, R. A. and Marshall, J. (2007). Atmosphere, Ocean, and Climate Dynamics. Academic Press.

Polhamus, E. C. (1984). A Review of Some Reynolds Number Effects related to Bodies at High Angles of Attack. Technical report, NASA CR 3809.

Pugh, D. (2004). Tides, Surges and Mean Sea-Level. JOHN WILEY and SONS.

Ralston, D. and Stacey, M. (2006). Shear and turbulence production across subtidal channels. 64, 147-171. 
Reiso, M., Muskulus, M. and Moe, G. (2011). Tower shadow - experiment comparing wake behind tubular and truss towers. In Proceedings of the 21 st International Offshore (Ocean) and Polar Engineering Conference. 157 - 164.

RenewableUK (2013). Wave and Tidal Energy in the UK. Technical report, London: RenewableUK.

Roberts, A., Thomas, B., Sewell, P., Khan, Z., Balmain, S. and Gillman, J. (2016). Current tidal power technologies and their suitability for applications in coastal and marine areas. Journal of Ocean Engineering and Marine Energy, 2, 227-245.

Roshko, A. (1961). Experiments on the flow past a circular cylinder at very high Reynolds number. Journal of Fluid Mechanics, 10, 345-356. ISSN 1469-7645.

Sarpkaya, T. and Isaacson, M. (1981). Mechanics of Wave Forces on Offshore Structures. Van Nostrand Reinhold International.

Schluntz, J. and Willden, R. (2015a). The effect of blockage on tidal turbine rotor design and performance. Renewable Energy, 81, 432-441.

Schluntz, J. and Willden, R. H. J. (2015b). An actuator line method with novel blade flow field coupling based on potential flow equivalence. Wind Energy, 18, 1469-1485.

Sescu, A. and Andersen, B. (2011). Computational investigation of tower shadow effects on wind turbines. In Proceedings of the ASME 2011 International Mechanical Engineering Congress and Exposition. 87-96.

Severn Tidal Power (2010). Severn Tidal Power - Feasibility Study Conclusions and Summary Report. Technical report, Severn Tidal Power. 
Shives, M. and Crawford, C. (2017). Tuned actuator disk approach for predicting tidal turbine performance with wake interaction. International Journal of Marine Energy, 17, 1 - 20. ISSN 2214-1669.

Smeaton, M., Vennell, R. and Harang, A. (2016). The effect of channel constriction on the potential for tidal stream power. Renewable Energy, 99, 45 - 56. ISSN 0960-1481.

Sorensen, J. N. and Shen, W. Z. (2002). Numerical Modeling of Wind Turbine Wakes. Journal of Fluids Engineering, 124, 393-399.

Sorensen, N. N., Michelsen, J. A. and Schreck, S. (2002). NavierâĂŞStokes predictions of the NREL phase VI rotor in the NASA Ames $80 \mathrm{ft} \tilde{\mathrm{A} U} \mathrm{U} 120 \mathrm{ft}$ wind tunnel. Wind Energy, 5, 151-169. ISSN 1099-1824.

Spalart, P. R. and Allmaras, S. R. (1992). A One-Equation Turbulence Model for Aerodynamic Flows. AIAA Journal, 14, $443-468$.

Sweby, P. K. (1984). High resolution schemes using flux limiters for hyperbolic conservation laws. SIAM J. Numer. Analysis, 21, 995-1011.

Tennekes, H. and Lumley, J. L. (1972). A First Course in Turbulence. MIT Press.

Turnock, S. R., Phillips, A. B., Banks, J. and Nicholls-Lee, R. (2011). Modelling tidal current turbine wakes using a coupled RANS-BEMT approach as a tool for analysing power capture of arrays of turbines. Ocean Engineering, 38, 1300 - 1307. ISSN 00298018.

van Kuik, G. A. (2007). The Lanchester-Betz-Joukowsky limit. Wind Energy, 10, 289291. ISSN 1099-1824. 
Vennell, R. (2010). Tuning turbines in a tidal channel. Journal of Fluid Mechanics, 663, $253-267$.

Vennell, R. (2012). The energetics of large tidal turbine arrays. Renewable Energy, 48, $210-219$.

Versteeg, H. K. and Malalasekera, W. (2007). An Introduction to Computational Fluid Dynamics. Pearson.

Vogel, C. (2014). Theoretical Limits to Tidal Stream Energy Extraction. Ph.D. thesis, University of Oxford, UK.

Walker, S. R. J. (2014). Hydrodynamic interactions of a tidal stream turbine and support structure. Ph.D. thesis, The University of Sheffield, UK.

Warschauer, K. and Leene, J. A. (1971). Experiments on mean and fluctuating pressures of circular cylinders at cross flow at very high Reynolds numbers. In Proc. Int. Conf. on Wind Effects on Buildings and Structures. 122-129.

Whelan, J. I., Graham, J. M. R. and Peiro, J. (2009). A free-surface and blockage correction for tidal turbines. Journal of Fluid Mechanics, 624, 281-291.

Wilcox, D. C. (1988). Re-assessment of the scale-determining equation for advanced turbulence models. AIAA Journal, 26, 1299-1310.

Wilcox, D. C. (2004). Turbulence Modeling for CFD. DCW Industries.

Williamson, C. H. K. (1988). The existence of two stages in the transition to threeâĂ $̌$ dimensionality of a cylinder wake. Physics of Fluids, 31, 3165-3168. 
Wimshurst, A. and Willden, R. H. J. (2016). Extracting lift and drag polars from bladeresolved computational fluid dynamics for use in actuator line modelling of horizontal axis turbines. Wind Energy, 20, 815-833.

Zdravkovich, M. M. (1997). Flow Around Circular Cylinders Volume I: Fundamentals. Oxford University Press. 